\title{
Hydrologic, Sediment, and Biological Data Associated with Irrigation Drainage in the Middle Green River Basin, Utah and Colorado, Water Years 1991-2000
}

By Ryan C. Rowland, David V. Allen, and Doyle W. Stephens, U.S. Geological Survey James W. Yahnke, Bureau of Reclamation

Nathan L. Darnall and Bruce Waddell, U.S. Fish and Wildlife Service

U.S. GEOLOGICAL SURVEY

Open-File Report 02-343

Prepared as part of the National Irrigation Drainage Water-Quality

Program

U.S. GEOLOGICAL SURVEY

BUREAU OF RECLAMATION

U.S. FISH AND WILDLIFE SERVICE

BUREAU OF INDIAN AFFAIRS

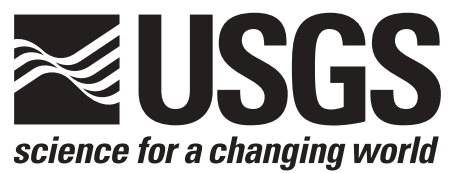

Salt Lake City, Utah

2002 


\section{U.S. DEPARTMENT OF THE INTERIOR}

GALE A. NORTON, Secretary

\section{U.S. GEOLOGICAL SURVEY}

Charles G. Groat, Director

Any use of trade, product, or firm names in this publication is for descriptive purposes only and does not imply endorsement by the U.S. Government.

For additional information write to:

Copies of this report can be purchased from:

District Chief

U.S. Geological Survey

2329 West Orton Circle

Salt Lake City, Utah 84119

http://ut.water.usgs.gov
U.S. Geological Survey

Branch of Information Services

Building 810

Box 25286, Federal Center

Denver, CO 80225-0286 


\section{CONTENTS}

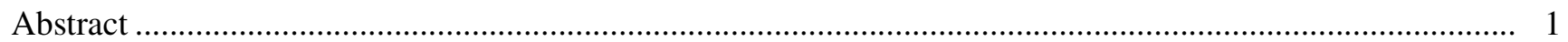

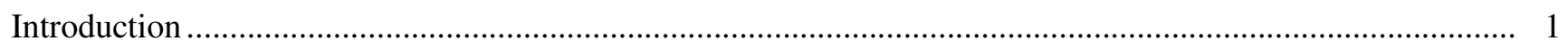

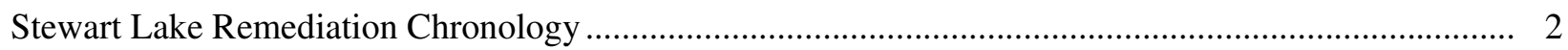

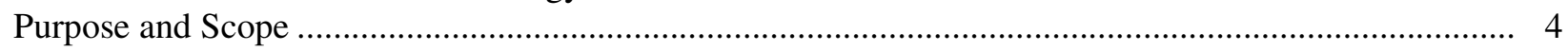

Physical and Chemical Data for Hydrologic Samples .............................................................. 5

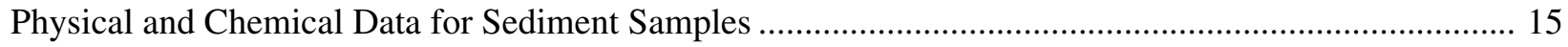

Physical and Chemical Data for Biological Samples....................................................................... 15

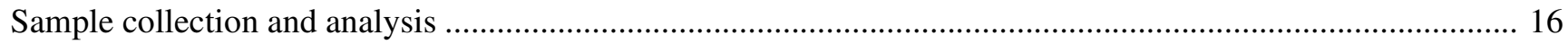

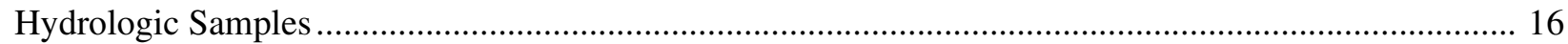

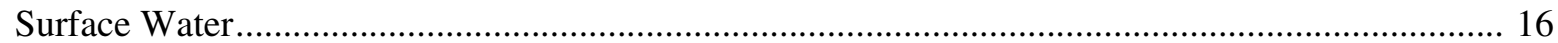

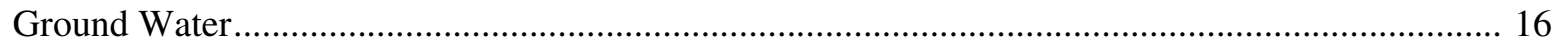

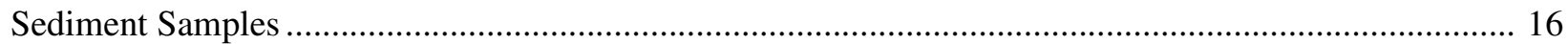

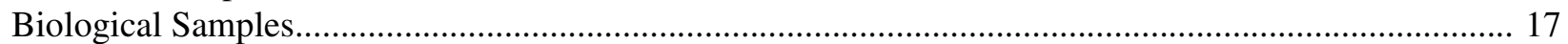

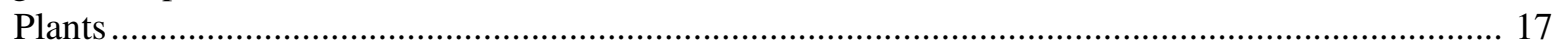

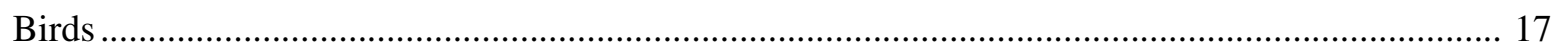

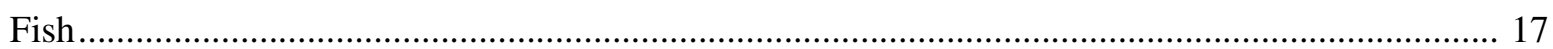

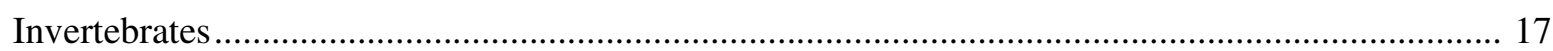

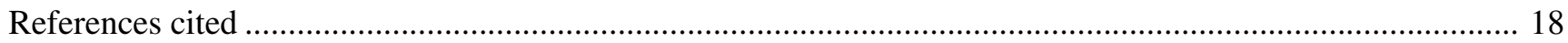




\section{FIGURES}

Figure 1. Map showing location of National Wildlife Refuges, Waterfowl Management Areas, wetlands, and surface-water sampling sites in the middle Green River basin, eastern Utah............ 3

Figure 2 Map showing location of surface-water sampling sites along the Brush Creek drainage, eastern Utah

Figure 3 Map showing location of Vernal sewage lagoons, subsurface irrigation drains, and surfacewater sampling sites along Ashley Creek drainage, eastern Utah

Figure 4 Map showing location of drains, wells, and water-quality sampling sites in Stewart Lake Waterfowl Management Area, eastern Utah.

Figure 5 Map showing location of sediment sampling sites sampled by the Bureau of Reclamation in and near Stewart Lake Waterfowl Management Area and the Green River, eastern Utah

Figure 6 Map showing location of bottom-sediment sampling sites sampled by the U.S. Geological Survey and Bureau of Reclamation in and near Stewart Lake Waterfowl Management Area, eastern Utah.

Figure 7 Map showing location of bottom-sediment sampling sites sampled by the U.S. Geological Survey and Bureau of Reclamation between irrigation drains J3 and J4 near Stewart Lake Waterfowl Management Area, eastern Utah, July 1994

Figure 8 Map showing location of bottom-sediment sampling sites sampled by the U.S. Geological Survey and Bureau of Reclamation between drains J3 and J2 near Stewart Lake Waterfowl Management Area, eastern Utah, June 1995

Figure 9 Map showing location of biological-sampling sites in and near Stewart Lake Waterfowl Management Area and selected sites in the middle Green River basin, eastern Utah.

Figure 10 Map showing location of biological-sampling sites in and near the Ouray National Wildlife Refuge, and selected sites in the middle Green River basin, eastern Utah. 


\section{TABLES}

Table 1. Hydrologic sampling-site designations, Bureau of Reclamation identifier, U.S. Geological Survey site identification number, and site type for data-collection sites in the middle Green River basin, Utah and Colorado

Table 2. Site number and site ID for bottom-sediment and suspended-sediment-samplng sites sampled by the Bureau of Reclamation, the U.S. Fish and Wildlife Service, and the U.S. Geological Survey at and near Stewart Lake Waterfowl Management Area, middle Green River basin, eastern Utah.. 22

Table 3. Site name and abbreviated site name for biological-sampling sites sampled by the U.S. Fish and Wildlife Service, middle Green River basin, eastern Utah

Table 4. Physical properties and chemical analyses for quality-control samples collected during 1993-96 from the middle Green River basin, Utah and Colorado

Table 5. Discharge, physical properties, and selenium concentration of water from the Green River and selected tributaries, Utah and Colorado, water years 1991-2000 . 28

Table 6. Discharge, physical properties, selenium concentration, and depth of water at selected sample locations in Brush Creek drainage near Jensen, Utah, water years 1991-2000.

Table 7. Discharge, physical properties, and selected chemical analyses of surface-water inflow to Stewart Lake near Jensen, Utah, water years 1991-2000

Table 8. Nitrogen concentration of surface-water inflow to Stewart Lake near Jensen, Utah, 1991 and 199456

Table 9. Concentration of selected radiochemicals in water from irrigation drains J3 and J4 near Jensen, Utah, 1992 56

Table 10. Physical properties and chemical analyses of water from wells in the Stewart Lake Waterfowl Management Area near Jensen, Utah, water years 1996-2000. 58

Table 11. Water levels for selected wells in the Stewart Lake Waterfowl Management Area near Jensen, Utah, water years 1996-2000 66

Table 12. Pedologic descriptions of borehole samples collected during installation of six monitoring wells in the Stewart Lake Waterfowl Management Area near Jensen, Utah, February 1996

Table 13. Summary of discharge, physical properties, selenium concentration, and dissolved-solids concentration of water in Ashley Creek and selected tributaries near Vernal and Naples, Utah, water years 1991-2000

Table 14. Discharge, physical properties, and chemical analyses of water from ponds, canals, and drains in the Ashley Creek drainage near Vernal and Naples, Utah, 1991-93....

Table 15. Discharge, physical properties, and chemical and isotopic analyses of water from lagoons and seeps in the vicinity of the Vernal Sewage Lagoons and Winter Storage Pond near Naples, Utah, 1991-95

Table 16. Concentration of selected radiochemicals in bottom-sediment samples collected near irrigation drains J3 and J4 in the Stewart Lake Waterfowl Management Area near Jensen, Utah, 1992......... 75

Table 17. Concentration of selenium in bottom-sediment samples collected in and near Stewart Lake Waterfowl Management Area near Jensen, Utah, July 1994. 76

Table 18. Concentration of selenium in soil and bottom-sediment samples collected from the east side of Stewart Lake Waterfowl Management Area near Jensen, Utah, June 1995.

Table 19. Concentration of selenium in bottom-sediment samples collected near irrigation drains J3 and J4 in the Stewart Lake Waterfowl Management Area near Jensen, Utah, June 1995.

Table 20. Concentration of selenium in bottom-sediment samples collected from Stewart Lake Waterfowl Management Area near Jensen, Utah, August 1995 
Table 21. Concentration of selenium species in bottom-sediment samples collected from Stewart Lake Waterfowl Management Area near Jensen, Utah, April 1996

Table 22. Concentration of total selenium in suspended-sediment samples collected from Stewart Lake Waterfowl Management Area near Jensen, Utah, May 1997

Table 23. Concentration of selenium in bottom-sediment samples collected from Green River backwaters near Stewart Lake Waterfowl Management Area near Jensen, Utah, July 1997 to July 1999.

Table 24. Concentration of selenium in bottom-sediment samples collected from Stewart Lake Waterfowl

Management Area near Jensen, Utah, July 1997-December 2000.

Table 25. Concentration of selenium species in bottom-sediment samples collected from experimental test plots and selected sites at Stewart Lake near Jensen, Utah, April 2000-August 2000.

Table 26. Concentration of total selenium in bottom-sediment samples collected from experimental test plots and selected sites at Stewart Lake near Jensen, Utah, April 2000-December 2000

Table 27. Trace-element concentration in whole body tissue composite samples of common carp collected from the Green River near Jensen, Utah, 1995

Table 28. Concentration of selenium in whole body tissue composite samples of common carp collected from the Green River near Jensen, Utah, 1996-2000.

Table 29. Selenium concentration in crayfish and fish samples collected from Green River backwaters near Stewart Lake Waterfowl Management Area near Jensen, Utah, 1997-2000 ....

Table 30. Concentration of selenium in common carp samples collected from Stewart Lake near Jensen, Utah, 1991-2000.

Table 31. Concentration of selenium in samples of small fish collected from Stewart Lake Waterfowl Management Area near Jensen, Utah, 1994-2000

Table 32. Concentration of selenium in nonendangered fish samples except common carp collected from Stewart Lake Waterfowl Management Area near Jensen, Utah, 1994-2000 102

Table 33. Concentration of selenium in bird eggs collected from Stewart Lake Waterfowl Management Area near Jensen, Utah, 1995-2000

Table 34. Concentration of selenium in bird livers collected from Stewart Lake Waterfowl Management Area near Jensen, Utah, 1995

Table 35. Concentration of trace elements in biological samples collected from selected sites in the middle Green River basin, eastern Utah (except Stewart Lake Waterfowl Management Area), 1991-93

Table 36. Concentration of selenium in muscle plugs from endangered fish from Stewart Lake Waterfowl

Management Area near Jensen, Utah, 1997-2000 .

Table 37. Concentration of selenium in muscle plugs from endangered fish from selected sites in the middle Green River basin, eastern Utah (except Stewart Lake Waterfowl Management Area), $1995-2000$ 


\section{CONVERSION FACTORS, DATUMS, AND ABBREVIATED WATER-OUALITY UNITS}

\begin{tabular}{|c|c|c|}
\hline Multiply & 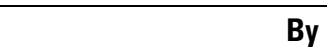 & To obtain \\
\hline cubic foot per second $\left(\mathrm{ft}^{3} / \mathrm{s}\right)$ & 0.02832 & cubic meter per second \\
\hline foot $(\mathrm{ft})$ & 0.3048 & meter \\
\hline gallon per minute (gal/min) & 0.06308 & liter per second \\
\hline inch (in.) & 25.4 & millimeter \\
\hline inch (in.) & 2.54 & centimeter \\
\hline mile $(\mathrm{mi})$ & 1.609 & kilometer \\
\hline square mile $\left(\mathrm{mi}^{2}\right)$ & 2.590 & square kilometer \\
\hline ton & 0.9072 & metric ton or megagram \\
\hline
\end{tabular}

Degree Celsius $\left({ }^{\circ} \mathrm{C}\right)$ may be converted to degree Fahrenheit $\left({ }^{\circ} \mathrm{F}\right)$ by using the following equation: ${ }^{\circ} \mathrm{F}=9 / 5\left({ }^{\circ} \mathrm{C}\right)+32$.

Degree Fahrenheit $\left({ }^{\circ} \mathrm{F}\right)$ may be converted to degree Celsius $\left({ }^{\circ} \mathrm{C}\right)$ by using the following equation:

$$
{ }^{\circ} \mathrm{C}=5 / 9\left({ }^{\circ} \mathrm{F}-32\right) \text {. }
$$

Vertical coordinate information is referenced to the North American Vertical Datum of 1988 (NAVD 88). Horizontal coordinate information is referenced to the North American Datum of 1983 (NAD 83).

Chemical concentration and water temperature are reported only in metric units. Chemical concentration in water is reported in milligrams per liter $(\mathrm{mg} / \mathrm{L})$ or micrograms per liter $(\mu \mathrm{g} / \mathrm{L})$. Milligrams per liter is a unit expressing the solute per unit volume (liter) of water. One thousand micrograms per liter is equivalent to 1 milligram per liter. For concentrations less than 7,000 milligrams per liter, the numerical value is about the same as for concentrations in parts per million. Stable isotope concentration is reported as permil, which is equivalent to parts per thousand. Radioactivity in water is expressed in picocuries per liter ( $\mathrm{pCi} / \mathrm{L}$ ), which is the amount of radioactive decay producing 2.2 disintegrations per minute in a unit volume (liter) of water. Radioactivity in sediment is expressed in picocuries per gram ( $\mathrm{pCi} / \mathrm{g}$ ), which is the amount of radioactive decay producing 2.2 disintegrations per minute in a unit mass (gram) of sediment. Specific conductance is reported in microsiemens per centimeter at 25 degrees Celsius $(\mu \mathrm{S} / \mathrm{cm})$. Chemical concentration in sediment is reported in micrograms per gram $(\mu \mathrm{g} / \mathrm{g})$, which is equal to parts per million. 
viii Conversion Factors 


\title{
Hydrologic, Sediment, and Biological Data Associated with Irrigation Drainage in the Middle Green River Basin, Utah and Colorado, Water Years 1991-2000
}

\author{
By Ryan C. Rowland, David V. Allen, and Doyle W. Stephens, U.S. Geological Survey \\ James W. Yahnke, Bureau of Reclamation \\ Nathan L. Darnall and Bruce Waddell, U.S. Fish and Wildlife Service
}

\section{ABSTRACT}

Hydrologic, sediment, and biological data were collected in the middle Green River basin in eastern Utah from 1991 to 2000 in an effort to monitor the effects of irrigation drainage on wetland areas and streams, aid in the development of remediation plans, and evaluate the effectiveness of selenium remediation efforts at Stewart Lake Waterfowl Management Area (WMA). Data consist primarily of selenium concentrations in surface water, ground water, bottom sediment, and biological samples. Supporting hydrologic data include field measurements of temperature, $\mathrm{pH}$, specific conductance, water levels in wells, and discharge at surface-water sites. Selected water samples also were analyzed for major ions, trace elements, nutrients, and gross alpha and beta radiation. The concentration of selected selenium species is reported for several bottom-sediment samples from Stewart Lake WMA and the concentration of total selenium in suspended-sediment samples from the area are included. Well logs for six wells installed at Stewart Lake WMA are presented along with trace-element data for several biological samples collected at selected sites throughout the middle Green River basin.

\section{INTRODUCTION}

During the last two decades, there has been increasing concern about the water quality of irrigation drainage, both surface and subsurface, and its potential effects on the health of humans, fish, and wildlife. In 1983, occurrences of mortality, birth defects, and reproductive failure in waterfowl were discovered by the U.S. Fish and Wildlife Service (USFWS) at the Kesterson National Wildlife Refuge (NWR) in western San Joaquin Valley, California. The unhealthy condition of waterfowl at Kesterson NWR was attributed to high selenium concentrations in irrigation drain water impounded in the refuge.

Because of concern that water-quality problems related to selenium or other trace inorganic and organic constituents in irrigation drainage might not be limited to the Kesterson NWR area, the U.S. Department of the Interior (DOI) began the National Irrigation Drainage Water-Quality Program (NIWQP) in 1985 to determine whether irrigation-related problems existed at other DOI-constructed or managed irrigation projects, NWRs, or other wetland areas for which the DOI has responsibility under the Migratory Bird Treaty Act, The Endangered Species Act, or other legislation. The program evolved into a five-phase process: (1) site identification, (2) reconnaissance investigations, (3) detailed studies, (4) planning for remediation, and (5) remediation. In the first three phases, the U.S.

Geological Survey (USGS) directed the activities of study teams composed of scientists from the USGS, the USFWS, the Bureau of Reclamation (BOR), and the Bureau of Indian Affairs (BIA). Activities for phases 4 and 5 are conducted by the agency that constructed the project, either BIA or BOR.

The NIWQP identified 26 areas in 14 states that warranted reconnaissance investigations, which have been completed (U.S. Department of the Interior, 2002). The investigations were conducted to determine whether irrigation drainage (1) has caused or has the potential to cause significant harmful effects on the health of humans, fish, and wildlife, or (2) may adversely affect the suitability of water for other uses. The reconnaissance investigations indicated there were 
sufficient problems to warrant further investigation at (1) Stillwater Wildlife Management Area, Nevada; (2) Salton Sea area, California; (3) middle Green River basin area, Utah, including Stewart Lake Wildlife Management Area (WMA) (Stephens and others, 1988); (4) Kendrick Project area, Wyoming; (5) Gunnison-Grand Valley areas, Colorado; (6) Sun River area, Montana; (7) San Juan River, New Mexico; and (8) Klamath Basin, California and Oregon. Detailed studies of each of these areas have been completed (U.S. Department of the Interior, 2002).

During the detailed study of the middle Green River basin (fig. 1), several areas were identified in which selenium was adversely affecting water quality and creating a hazard to wildlife (Stephens and others, 1992). For example, the median dissolved-selenium concentration in irrigation drain water that discharged to Stewart Lake WMA continually exceeded the State of Utah's standard for wildlife protection of $5 \mu \mathrm{g} / \mathrm{L}$ and was as high as $140 \mu \mathrm{g} / \mathrm{L}$. Dissolved-selenium concentrations in the discharge measured at the irrigation drains and the outflow from Stewart Lake during the detailed study showed that 75 percent of the selenium load from the drains was retained in Stewart Lake, presumably in the bottom sediment and biota. Concentrations of total selenium in bottom material collected near the outfall of the irrigation drains at Stewart Lake were as high as $250 \mu \mathrm{g} / \mathrm{g}$. Selenium concentrations in biota from Stewart Lake were high relative to concentrations measured at other sites in the middle Green River basin, resulting in low waterfowl nesting success and low populations of benthic insects. The elevated levels of selenium in water, sediment, and biota at Stewart Lake were of heightened concern because endangered fish that are endemic to the Green River, such as the razorback sucker (Xyrauchen texamus) and the Colorado pikeminnow (Ptychocheilus lucius), were known to use Stewart Lake as a rearing area. The source of the selenium in irrigation drain water at Stewart Lake WMA is irrigated soils derived from the Cretaceous-age Mancos Shale, a geologic formation known to contain elevated levels of selenium.

On the basis of findings in the detailed study of the middle Green River basin, an environmental assessment and alternatives for remediation of the Stewart Lake area were published (U.S. Department of the Interior, 1997). The NIWQP initiated remediation activities the same year. An inlet channel was excavated between Stewart Lake and the Green River in May 1997 to provide a temporary source of goodquality water during high stage events on the Green River. Stewart Lake was drained in July 1997 to dry the bottom sediment, discourage waterfowl from using the WMA, and prevent endangered fish, such as the razorback sucker, from migrating from the Green River to the lake during remediation activities. To prevent ponding of seepage water in the lake bottom during remediation activities, drain channels were excavated in October 1997. In November 1997 and June 1998, the subsurface irrigation drains that were the major source of selenium to Stewart Lake were rerouted around the lake and into the Green River. In May 1999, water-flow control structures were constructed at the new inlet channel and the outlet channel of Stewart Lake to aid in its future management. The infrastructure for a new water source for Stewart Lake will be completed in 2002. However, the new water source will not be used until efforts to reduce the concentration of selenium in the sediment are completed. These remediation actions are summarized in chronological order below. The reader will find it useful to refer to this chronology when viewing the data from Stewart Lake WMA in this report.

\section{Stewart Lake Remediation Chronology}

- May 1997-Inlet channel excavated between Stewart Lake and Green River.

- July 1997-Stewart Lake drained.

- October 1997-Drain channels excavated in lake bottom.

- November 1997-Subsurface irrigation drains J1 and J1a were combined and diverted around Stewart Lake to the Green River.

- June 1998-Subsurface irrigation drains J2, J3, and $\mathrm{J} 4$ were combined and diverted around Stewart Lake to the Green River.

- May 1999-Water-flow control structures were constructed at the inlet channel and outlet of Stewart Lake.

Beginning in the spring of 1997, annual flooding and draining of Stewart Lake with water from the Green River has been used to facilitate removal of selenium from bottom sediments in the WMA. This method of selenium remediation was chosen because of the relatively large decrease in selenium concentration in sediments following the 1997 flood 


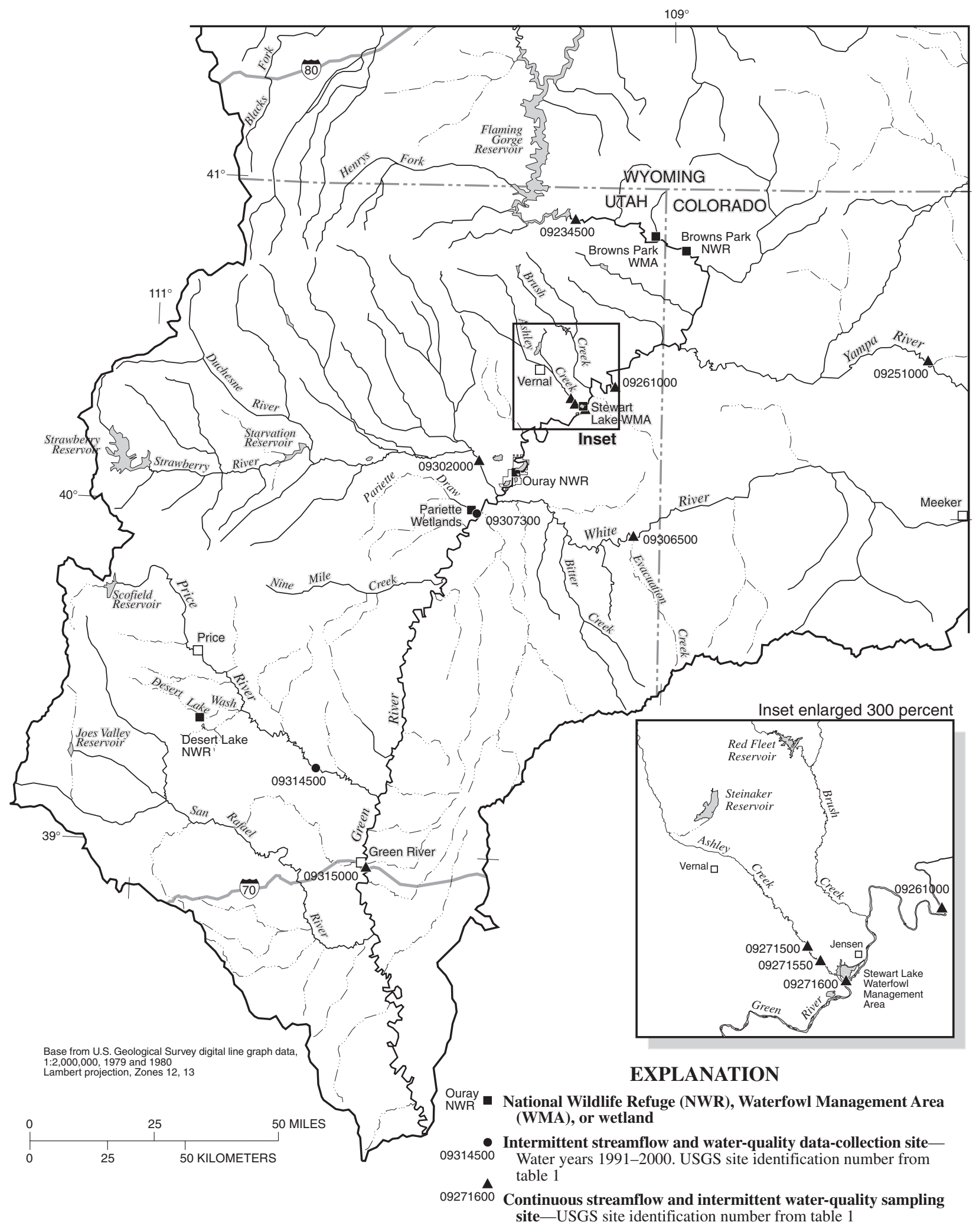

Figure 1. Location of National Wildlife Refuges, Waterfowl Management Areas, wetlands, and surface-water sampling sites in the middle Green River basin, eastern Utah. 
with water from the Green River. The theory behind the flood and drain cycle is that elemental selenium and adsorbed selenium, in the form of selenite, are oxidized when the lake is drained. The oxidized selenium, in the form of selenate, is then dissolved by flood water from the Green River and discharged to the Green River when the lake is drained. Generally, the lake was flooded from late April to early July and dry the rest of the year. In 1999, experimental test plots were established at three sites in Stewart Lake to discern if tilling the sediments prior to the annual flood cycle improved selenium removal. Each till plot was $100 \mathrm{ft}$ by $100 \mathrm{ft}$. Control plots with the same dimensions were established adjacent to each till plot. The control plots were left undisturbed. Within each till plot, an area $8 \mathrm{ft}$ by $8 \mathrm{ft}$ was framed with timber, lined with plastic, and filled with sediments from the till plot to a depth of approximately 6 in. The purpose of the small box plots was to provide a test area unaffected by shallow ground water during the flood cycle. Bottom-sediment data from the test plots are included in this report. Annual flooding and draining of Stewart Lake to remove selenium from bottom sediments will continue through the spring and early summer of 2002.

The detailed study of the middle Green River basin also identified high selenium concentrations in water and biota from Ashley Creek downstream from the sewage lagoons near Vernal, Utah (Stephens and others, 1992). During the study, the average concentration of selenium in water from lower Ashley Creek was $73 \mu \mathrm{g} / \mathrm{L}$ during the detailed study. Biota from Ashley Creek had selenium concentrations that ranked among the highest in the middle Green River basin. The source of the selenium was identified as selenium-laden shallow ground water and seepage that originated as leakage from the nearby sewage lagoons that overlie the Mancos Shale and discharged to Ashley Creek.

The elevated levels of selenium and high salinity in Ashley Creek below the Vernal sewage lagoons prompted the USFWS to urge the Ashley Valley Water Management District, the Utah Department of Environmental Quality, and the U.S. Environmental Protection Agency to take action to prevent seepage from the Vernal sewage lagoons from contaminating Ashley Creek (Stephens and Waddell, 1998). The sewage lagoons were decommissioned in April 2001 after a new wastewater treatment facility was constructed. The new facility utilizes a sealed oxidation ditch containing activated sludge to break down organic matter. Wastewater is then passed through secondary clarifiers and an ultraviolet disinfection system before it is discharged to Ashley Creek. Wastewater and treated wastewater do not come in contact with the Mancos Shale, eliminating the potential for selenium contamination. The USGS, in conjunction with the BOR Colorado River Basin Salinity Control Program, is monitoring Ashley Creek to evaluate water-quality improvements. Note that because the significant source of selenium to Ashley Creek was not related to DOI irrigation projects, NIWQP was not asked to participate in the Ashley Creek remediation activities described above.

Data collected for the detailed study of the middle Green River basin is contained in Peltz and Waddell (1991). Additional literature regarding irrigation drainage in the middle Green River basin is contained in Stephens and others (1988), Stephens and others (1991), Stephens (1992), and Stephens and Waddell (1998).

\section{Purpose and Scope}

This report presents the results of data collection in the middle Green River basin area in Utah and Colorado during water years 1991-2000, especially in the vicinity of Stewart Lake WMA. The middle Green River basin is operationally defined as an area of about $12,500 \mathrm{mi}^{2}$ that comprises the main stem of the Green River and its tributaries between Flaming Gorge Reservoir and the confluence of the Price and Green Rivers, about 15 mi north of the city of Green River, Utah (fig. 1). Included are the lower parts of the Yampa River and White River drainage basins, all of the Duchesne River and Price River drainage basins, and smaller tributaries such as Pariette Draw and Nine Mile Creek.

These data were collected to (1) further assess the effects of irrigation drainage on water quality, bottom sediments, and biota; (2) aid remediation planning in the middle Green River basin; and (3) assess the success of remediation actions at Stewart Lake WMA. Data consist primarily of selenium concentrations in surface-water, ground-water, bottomsediment, and biological samples. Additional waterrelated data include field measurements of temperature, $\mathrm{pH}$, specific conductance, water levels in wells, and discharge at surface-water sites. Data for selected water samples also include analyses of major ions, trace 
elements, nutrients, and gross alpha and beta radiation. The concentration of selected selenium species is reported for several bottom-sediment samples from Stewart Lake WMA and the concentration of total selenium in suspended-sediment samples from the area are included. Well logs for six wells installed at Stewart Lake WMA are presented along with trace-element data for several biological samples collected throughout the middle Green River basin.

The data-collection effort included (1) waterquality data from the Green River and select tributaries (fig. 1), Brush Creek drainage (fig. 2), Ashley Creek, Vernal sewage lagoons, and selected subsurface irrigation drains in the Vernal area (fig. 3), and Stewart Lake WMA (fig. 4); (2) bottom-sediment and suspended-sediment data collected from Stewart Lake WMA and the Green River (figs. 5, 6, 7, and 8); and (3) biological data collected from Stewart Lake WMA (fig. 9) and selected sites in the middle Green River basin (figs. 9 and 10).

Data are organized into tables of sample site information (tables 1-3); physical and chemical data for water samples collected from the middle Green River basin, including Stewart Lake WMA (tables 410 and 13-15); water-level data and well logs for wells at Stewart Lake WMA (tables 11 and 12, respectively); physical and chemical data for bottom-sediment and suspended-sediment samples collected in and near Stewart Lake WMA (tables 16-26); and biological data collected from Stewart Lake WMA and selected sites in the middle Green River basin (tables 27-37).

\section{Physical and Chemical Data for Hydrologic Samples}

Water-sampling site designations and identification numbers used by the BOR and USGS are cross-referenced in table 1. Water-sample collection sites are located by latitude and longitude. If more than one site exists at the same latitude and longitude, the USGS uses a sequential number to form a unique identifier for each site (table 1).

Water-quality data for water from the middle Green River are listed in tables 4-10. Results of analyses of quality-control water samples are contained in table 4 . The quality-control samples consisted of process blanks of inorganic-free water processed with sampling equipment in the field. The process blank sample collected on March 26, 1993, had a high selenium concentration $(32 \mu \mathrm{g} / \mathrm{L})$. The value was probably a result of miscommunication between a new employee and the USGS field technician regarding proper quality-assurance procedures at the time the sample was collected. Discharge, physical properties, and selenium concentrations for water from the Green River and select tributaries are shown in table 5. Data are presented for three sites on the Green River (Green River near Greendale, Utah; Green River near Jensen, Utah; and Green River near Green River, Utah); the Yampa River near Maybell, Colorado; two sites on Ashley Creek (Ashley Creek near Jensen, Utah, and Ashley Creek below the Union Canal diversion near Jensen, Utah); irrigation drain discharge above and below Stewart Lake WMA; the Stewart Lake outflow near Jensen, Utah; the Duchesne River near Randlett, Utah; the White River near Watson, Utah; Pariette Draw near Ouray, Utah; and finally, the Price River at Woodside, Utah. Discharge, physical properties, selenium concentrations, and depth of surface water (Red Fleet Reservoir) for water from selected sites in the Brush Creek drainage are shown in table 6. Data are presented for Big Brush Creek above and below Red Fleet Reservoir, Red Fleet Reservoir, several sites on Brush Creek, and several sites on Burns Bench canal, including several seeps that discharge to the canal.

Discharge, physical properties, and selected chemical analyses of surface-water inflow, including irrigation drains, to Stewart Lake WMA are shown in table 7. Note that irrigation drains J1 and J1A were combined and extended to the Green River in November 1997. Likewise, irrigation drains J2, J3, and J4 were combined and extended to the Green River in June 1998. Data from the inlet to Stewart Lake (constructed in May 1997), the North Collector Ditch (constructed in October 1997), and several seeps along the north edge of Stewart Lake are also contained in table 7. Nitrogen concentrations in water from irrigation drains $\mathrm{J} 1, \mathrm{~J} 1 \mathrm{~A}, \mathrm{~J} 2, \mathrm{~J} 3$, and $\mathrm{J} 4$ are shown in table 8 . The concentration of selected radiochemicals in water from irrigation drains $\mathrm{J} 3$ and $\mathrm{J} 4$ is shown in table 9. Physical properties and chemical analyses (including selenium) for water from wells located in the Stewart Lake WMA are shown in table 10. Water levels for selected wells in the Stewart Lake WMA are shown in table 11. Pedologic descriptions of borehole cuttings collected from wells installed in the Stewart Lake WMA are shown in table 12. Discharge, physical properties, selenium concentrations, and dissolvedsolids concentrations of water from Ashley Creek 


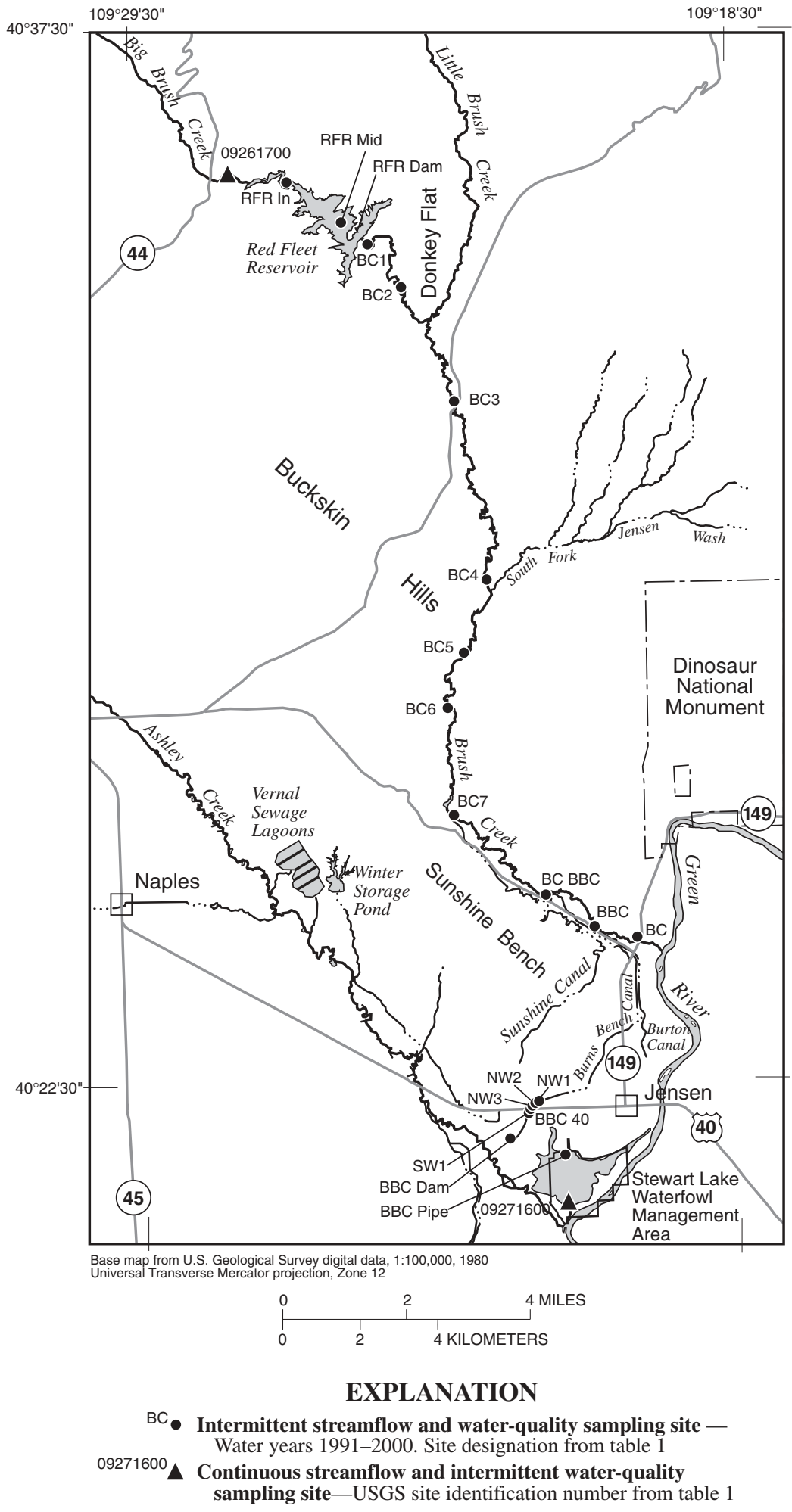

Figure 2. Location of surface-water sampling sites along the Brush Creek drainage, eastern Utah. 


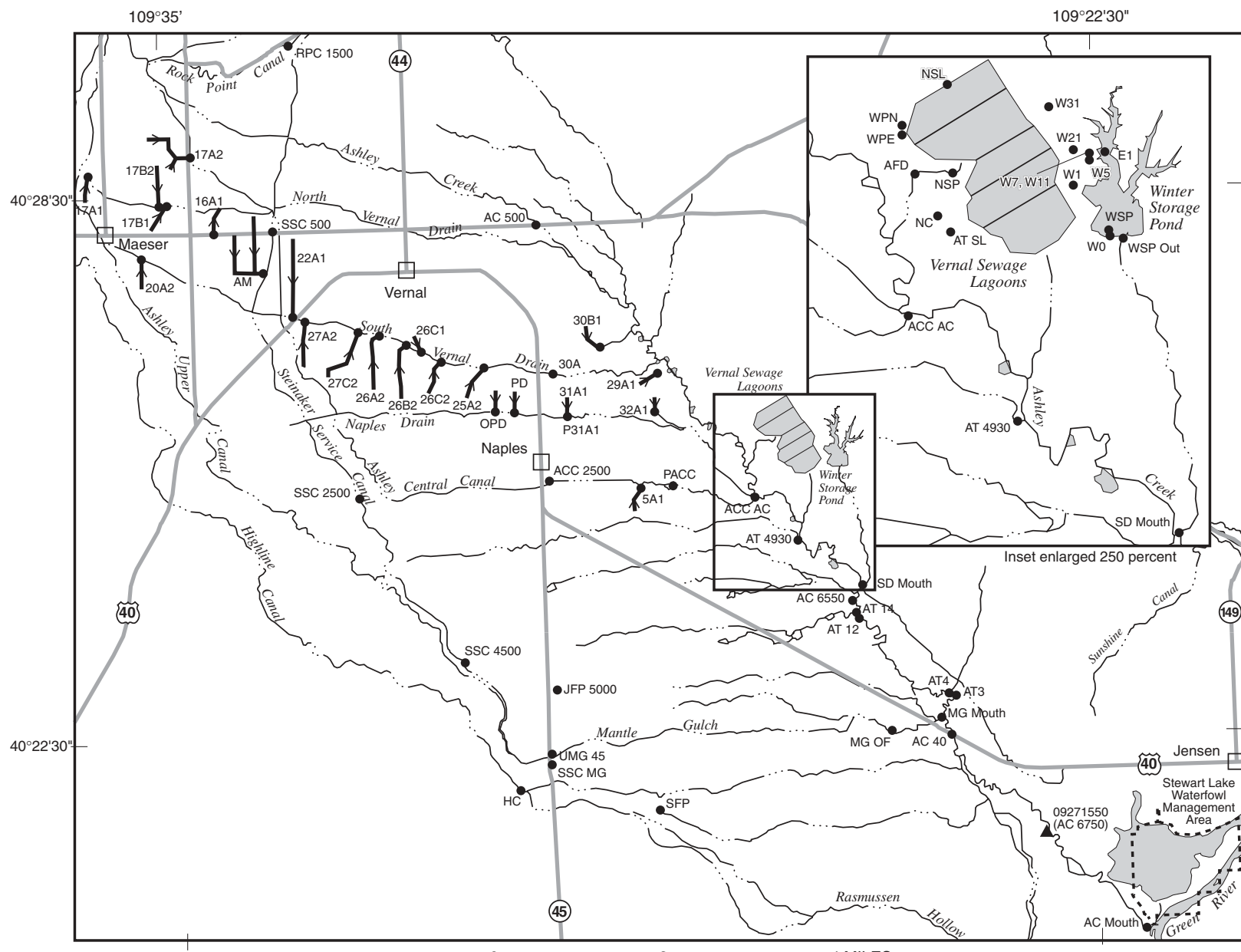

Base map from U.S. Geological Survey digital data, 1:100,000, 1980

4 MILES

$$
{ }_{0}
$$

4 KILOMETERS

\section{EXPLANATION}

$28 \mathrm{~A} 1 \rightarrow$ Drain and designation from table 1 -Arrow indicates direction of flow

$\mathrm{AC} 40 \bullet$ Physical and water-quality sampling site and designation from table 1

09271550 Continuous streamflow, intermittent water-quality sampling site-USGS site identification number and designation from table 1

Figure 3. Location of Vernal sewage lagoons, subsurface irrigation drains, and surface-water sampling sites along Ashley Creek drainage, eastern Utah. 


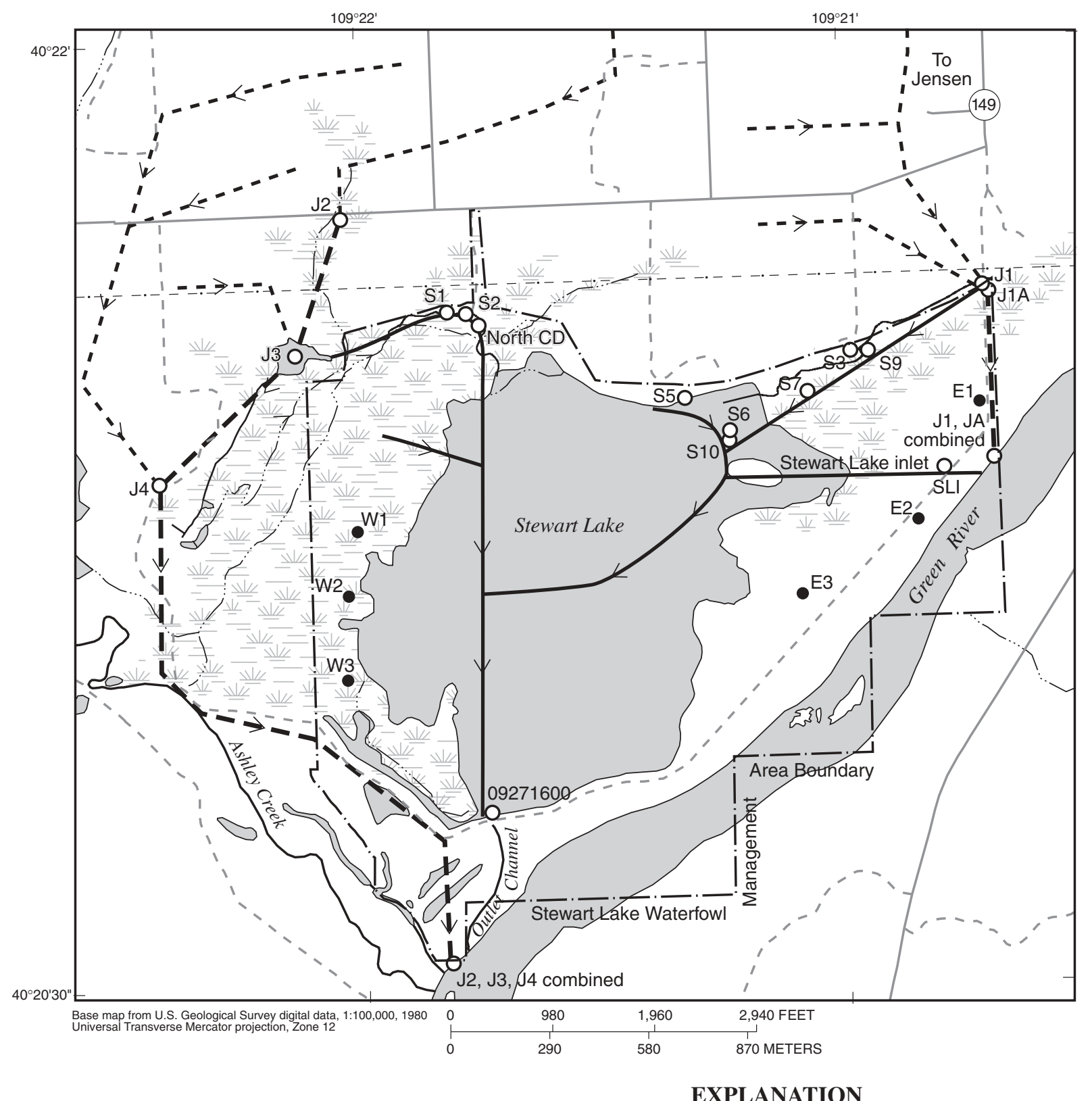

EXPLANATION

$-\rightarrow$ - Drain-Arrow indicates direction of flow

$\longrightarrow$ Drain extension, completed in November 1997-Arrow indicates direction of flow

$\longrightarrow$ Surface drain, excavated in November 1997—Arrow indicates direction of flow

E3-Well and designation from table 1

$\mathrm{J} 3 \mathrm{O}$ Intermittent, physical, and water-quality sampling site—Designation from table 1

Figure 4. Location of drains, wells, and water-quality sampling sites in Stewart Lake Waterfowl Management Area, eastern Utah. 


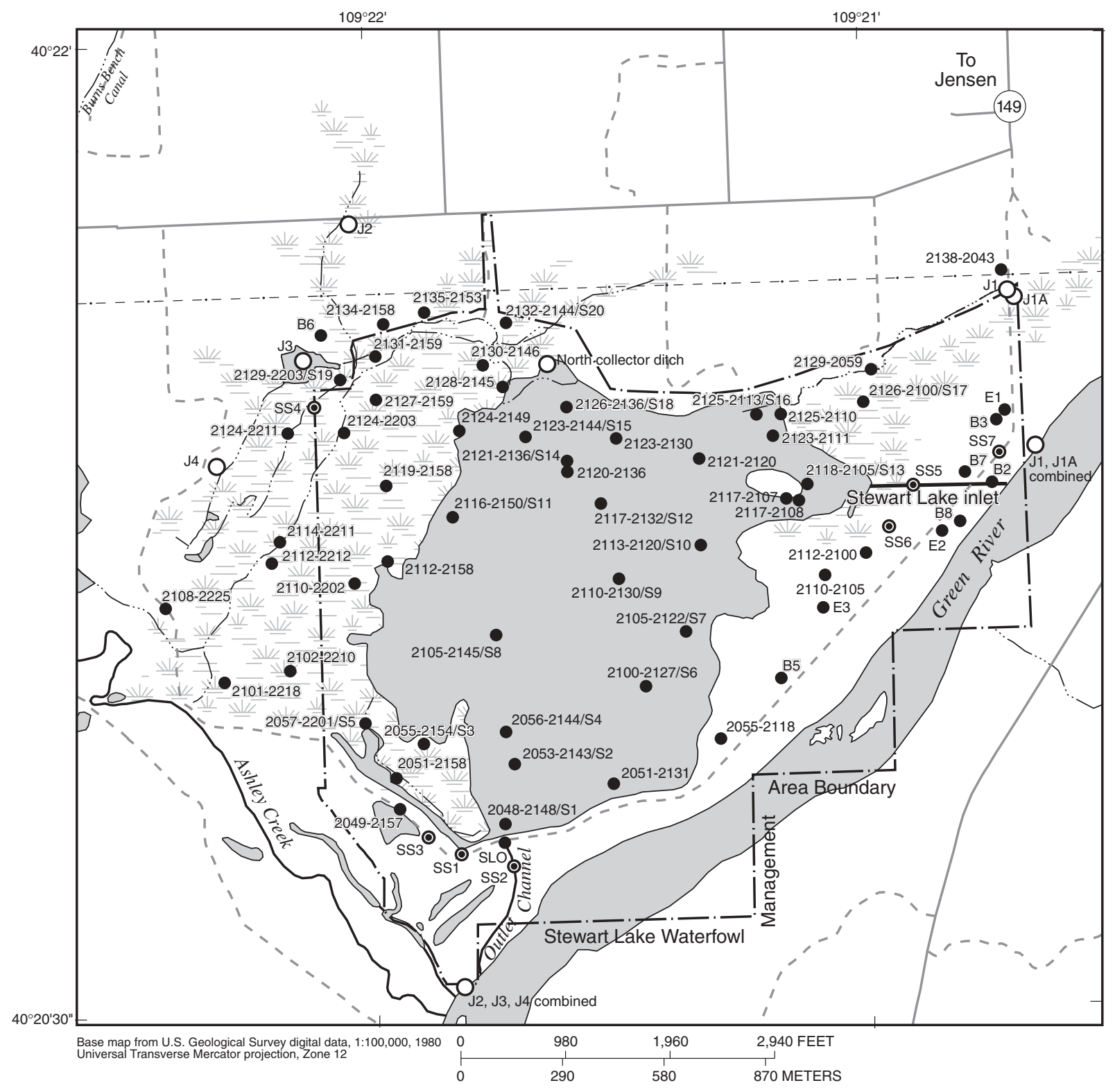

\section{EXPLANATION}

J3 $\mathrm{O}$ Intermittent, physical, and water-quality sampling site-Designation from table 1 2051-2131 Sediment-sample site-Designation from table 3

SS2 $\odot$ Suspended-sediment sample site-Designation from table 2

Figure 5. Location of sediment sampling sites sampled by the Bureau of Reclamation in and near Stewart Lake Waterfowl Management Area and the Green River, eastern Utah. 


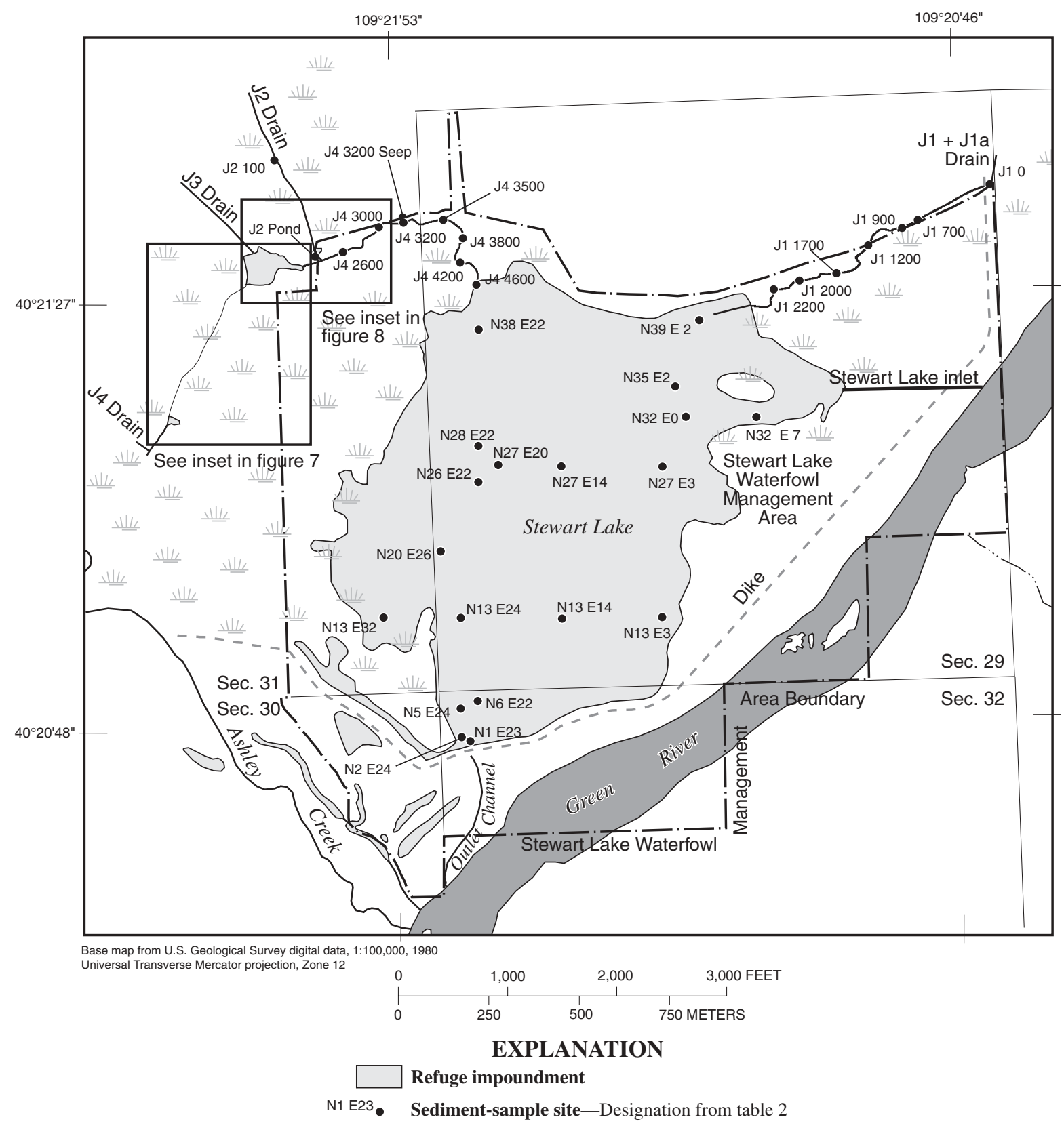

Figure 6. Location of bottom-sediment sampling sites sampled by the U.S. Geological Survey and Bureau of Reclamation in and near Stewart Lake Waterfowl Management Area, eastern Utah. 


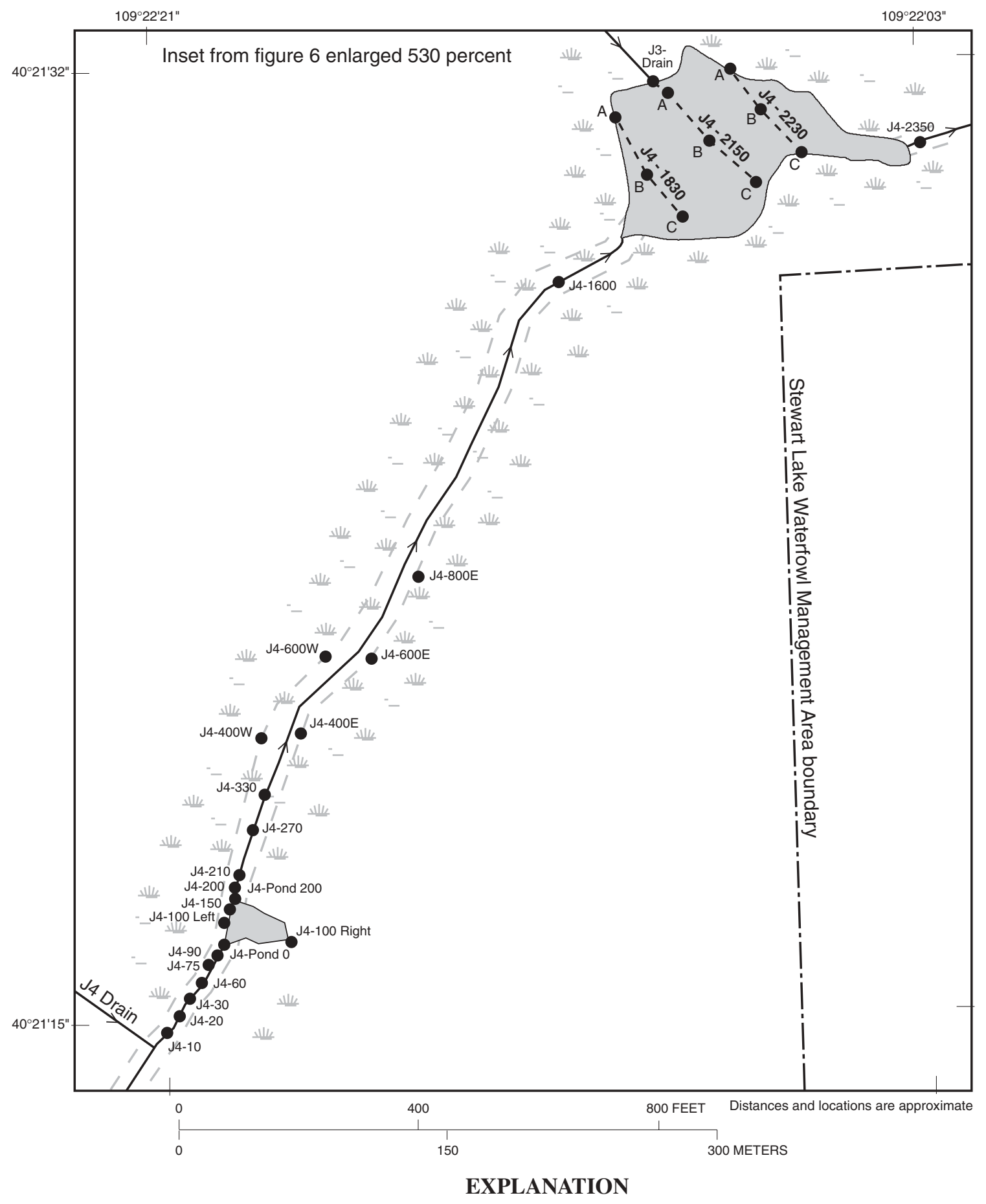

\footnotetext{
$\rightarrow$ Drain-Arrow indicates direction of flow

- - - Line of cross section

${ }^{\mathrm{J} 4-10}$ Sediment-sample site-Designation from table 3
}

Figure 7. Location of bottom-sediment sampling sites sampled by the U.S. Geological Survey and Bureau of Reclamation between irrigation drains J3 and J4 near Stewart Lake Waterfowl Management Area, eastern Utah, July 1994. 


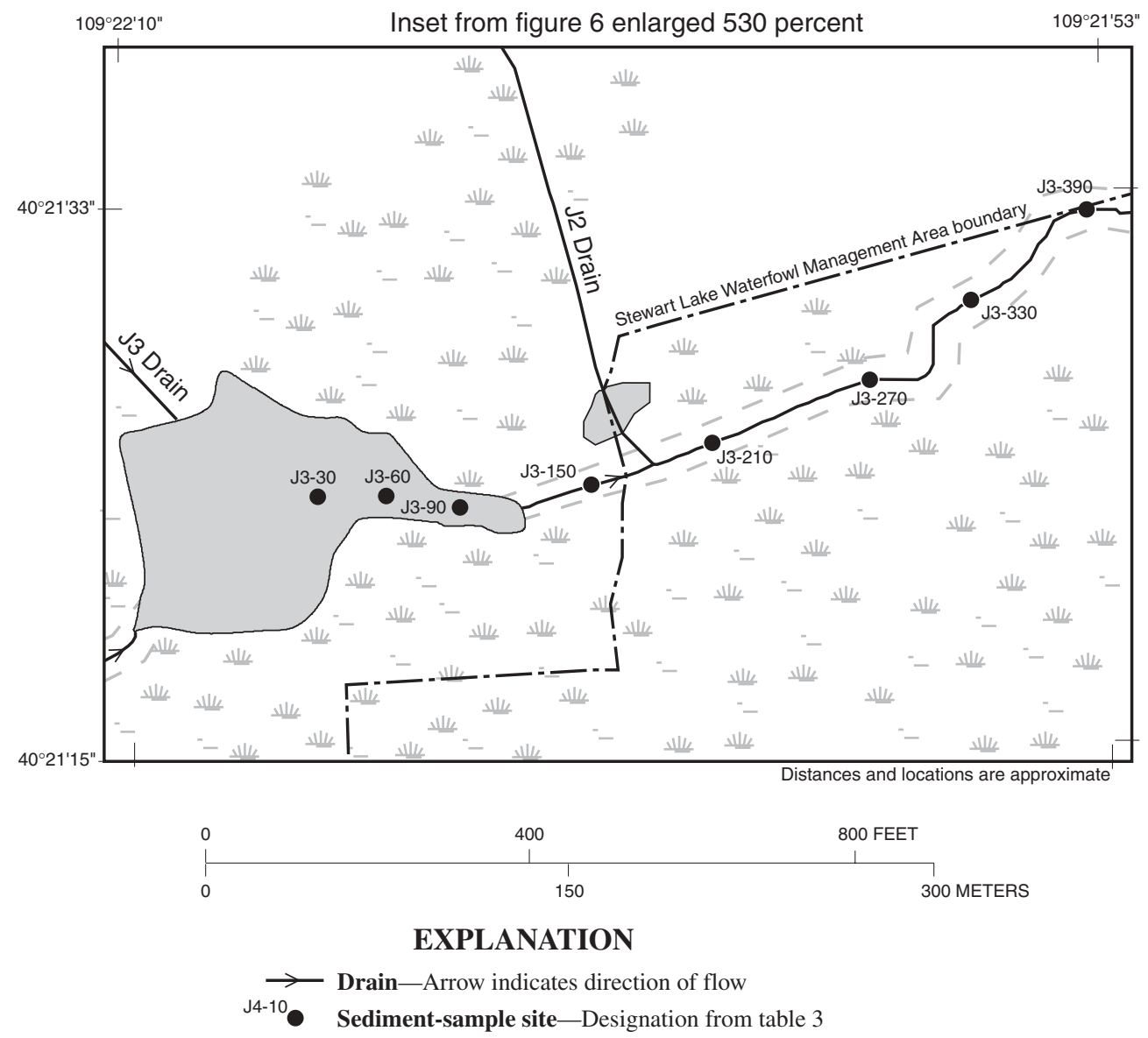

Figure 8. Location of bottom-sediment sampling sites sampled by the U.S. Geological Survey and Bureau of Reclamation between drains J3 and J2 near Stewart Lake Waterfowl Management Area, eastern Utah, June 1995. 


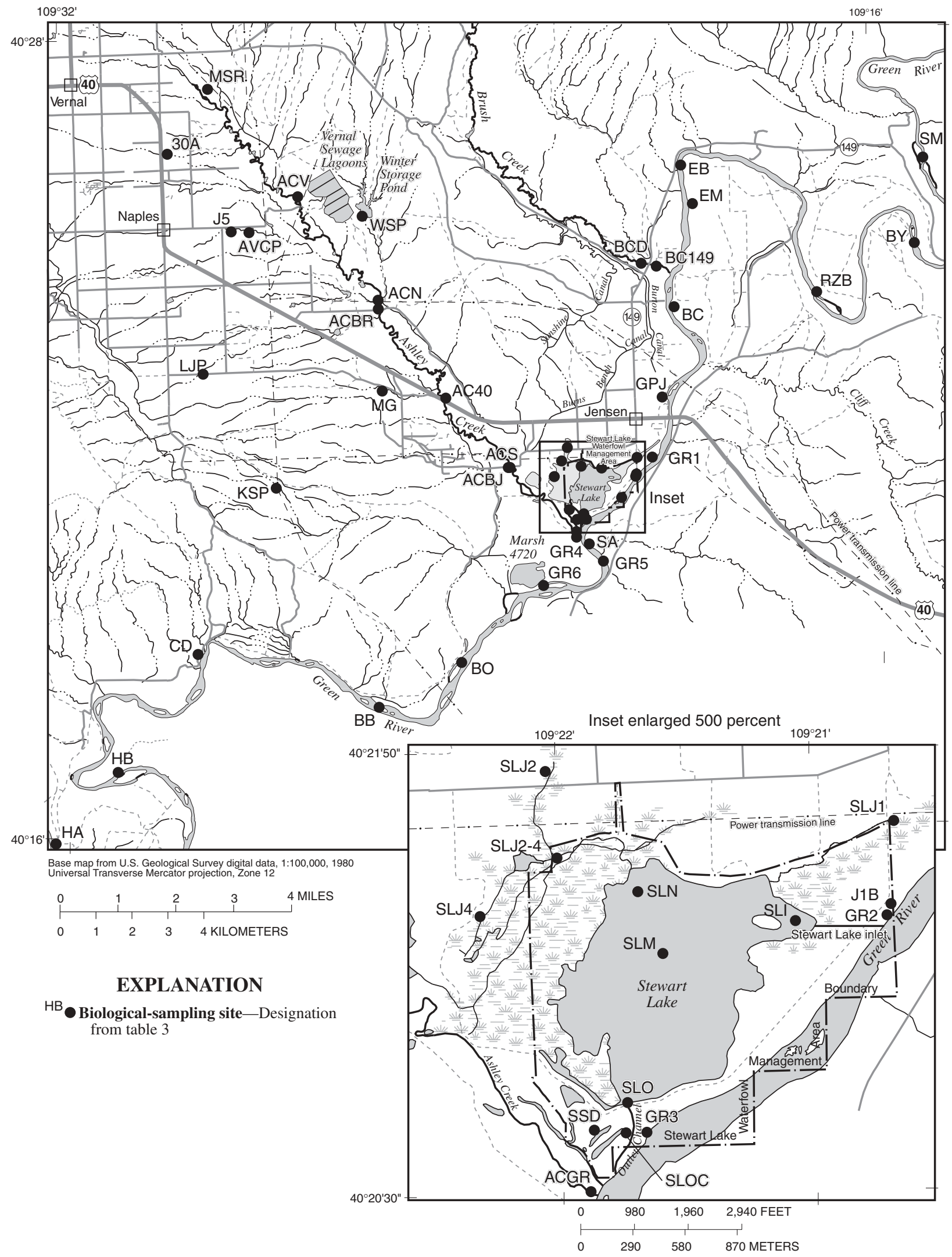

Figure 9. Location of biological-sampling sites in and near Stewart Lake Waterfowl Management Area and selected sites in the middle Green River basin, eastern Utah. 


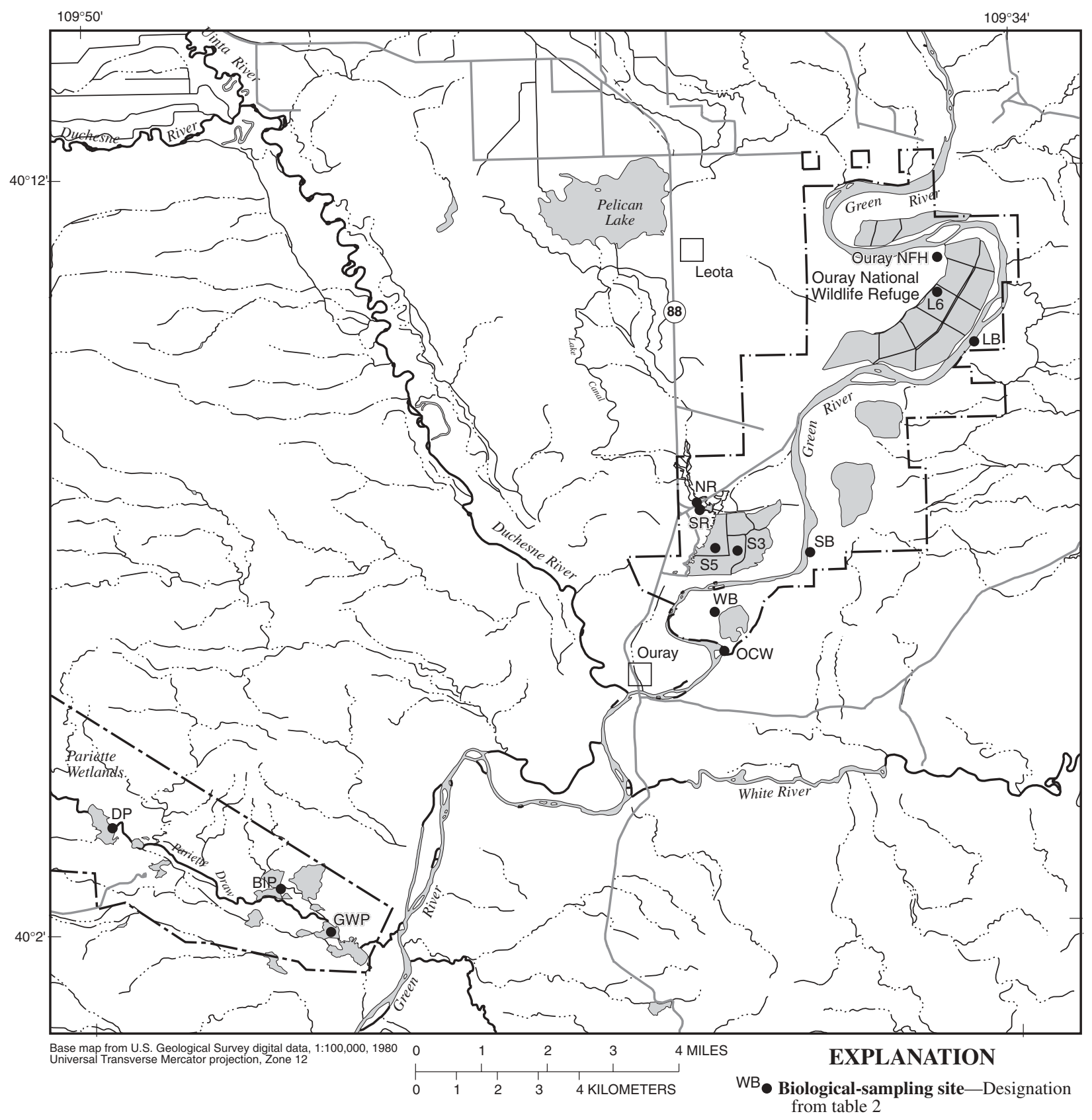

Figure 10. Location of biological-sampling sites in and near the Ouray National Wildlife Refuge, and selected sites in the middle Green River basin, eastern Utah. 
and selected tributaries near Vernal and Naples, Utah, are shown in table 13. Discharge, physical properties, and chemical analyses of water from ponds, canals, and drains in the Ashley Creek drainage are shown in table 14. Discharge, physical properties, and chemical analyses of water from seeps and sewage lagoons in the vicinity of Vernal and Naples, Utah, are shown in table 15 .

\section{Physical and Chemical Data for Sediment Samples}

Sediment-sampling sites sampled by the BOR, the USFWS, and the USGS are listed in table 2, which correlates each site with a location figure in this report. Physical and chemical data for bottom-sediment and suspended-sediment samples collected from Stewart Lake WMA and the Green River adjacent to Stewart Lake are shown in tables 16-26. Analyses of selected radiochemicals in sediment samples collected in 1992 near the discharge points of irrigation drains $\mathrm{J} 3$ and $\mathrm{J} 4$ are shown in table 16. The concentration of total selenium in bottom-sediment samples collected in July 1994 are shown in table 17. Total selenium concentrations in bottom-sediment samples collected from upland areas adjacent to Stewart Lake WMA in June 1995 are shown in table 18. Total selenium concentrations in bottom-sediment samples collected near irrigation drains $\mathrm{J} 3$ and $\mathrm{J} 4$ discharge points in June 1995 are shown in table 19. Total selenium concentrations in bottom-sediment samples collected from Stewart Lake WMA in August 1995 are shown in table 20. Concentrations of selected selenium species in bottom-sediment samples collected from Stewart Lake WMA in April 1996 are shown in table 21. The concentration of total selenium in suspended-sediment samples from Stewart Lake WMA and the Green River are shown in table 22. Total selenium concentrations in bottom-sediment samples collected from the Green River at selected sites adjacent to Stewart Lake WMA between July 1997 and July 1999 are shown in table 23. Total selenium concentrations in bottom-sediment samples collected from selected sites in the Stewart Lake WMA between July 1997 and December 2000 are shown in table 24. Concentrations of selenium species in bottom-sediment samples collected from experimental test plots and selected sample sites in the Stewart Lake WMA between April 2000 and August 2000 are shown in table 25. Total concentration of selenium in bottom-sediment samples collected from experimental test plots and selected sites in the Stewart Lake WMA between April 2000 and December 2000 are shown in table 26.

\section{Physical and Chemical Data for Biological Samples}

Biological-sampling sites sampled by the USFWS are listed in table 3, which correlates each site to a location figure in this report. Biological data are shown in tables 27-34. Trace-element concentrations in common carp collected from the Green River near Jensen, Utah, in 1995 are shown in table 27. Selenium concentrations in common carp collected from the Green River near Jensen, Utah, between 1996 and 2000 are shown in table 28. Selenium concentrations in crayfish and fish samples collected from Green River backwaters adjacent to Stewart Lake WMA from 1997 to 2000 are shown in table 29. Selenium concentrations in composite samples of carp collected in the Stewart Lake WMA between 1991 and 2000 are shown in table 30. Selenium concentrations in small fish collected from Stewart Lake WMA between 1994 and 2000 are shown in table 31. Selenium concentrations in large fish collected from Stewart Lake between 1994 and 2000 are shown in table 32. Selenium concentrations in bird eggs collected from Stewart Lake WMA between 1995 and 2000 are shown in table 33. Selenium concentrations in bird livers collected from Stewart Lake WMA in 1995 are shown in table 34. Traceelement concentrations in fish, birds, bird eggs, plants, and invertebrates at selected sites in the middle Green River basin, except for Stewart Lake WMA, are shown in table 35. The concentration of selenium in muscle plugs from endangered fish captured and released at Stewart Lake WMA between 1997 and 2000 are shown in table 36. The concentration of selenium in muscle plugs from endangered fish captured and released at selected sites in the middle Green River basin between 1995 and 2000 are shown in table 37. 


\section{SAMPLE COLLECTION AND ANALYSIS}

\section{Hydrologic Samples}

\section{Surface Water}

Surface-water samples were collected and processed by using a modification of the trace element protocol developed by the USGS. Where water was sampled from wide streams such as the Green River or sources not known to be well mixed, a DH48-TM sampler (U.S. Geological Survey, 1977) was employed with equal-width depth-integrated procedures. Field measurements of discharge, water temperature, $\mathrm{pH}$, and specific conductance were made at the time the samples were collected. Samples were filtered in the field with 0.45 -micrometer porosity cartridge or plate filters, acidified with nitric acid where necessary, and submitted to the USGS National Water Quality Laboratory (NWQL) in Arvada or Denver, Colorado (the NWQL moved to Denver in April 1999).

Analytical methods used at the NWQL are described in Techniques of Water-Resources Investigations of the U.S. Geological Survey (Fishman and Friedman, 1989).

\section{Ground Water}

Ground-water samples were collected and processed according to the procedures described in Techniques of Water-Resources Investigations of the U.S. Geological Survey (Wilde and Radtke, 1999). Depending on the well, a peristaltic pump, WaTerra inertial pump, or a dedicated bailer was used to purge and collect samples from the well. Three casing volumes of water were purged from each well, or the well was pumped dry and allowed to recover prior to sample collection. Parameters such as temperature, $\mathrm{pH}$, and specific conductance were measured in the field. Samples were filtered in the field with 0.45 -micrometer porosity cartridge or plate filters, acidified with nitric acid where necessary, and submitted to the NWQL.

\section{Sediment Samples}

A variety of methods were used to collect bottom-material samples from Stewart Lake WMA. In July 1994, bottom-material samples were collected with a BMH-53 sampler (U.S. Geological Survey,
1977, p. 3-37). Samples were homogenized and pebbles and large stems removed, but the sediment was not sieved. In June 1995, a truck-mounted Giddings hydraulic drill rig was used to collect sediment from upland areas adjacent to Stewart Lake, and hand augers or polycarbonate tubes were used at areas in the lake bottom. Between 1996 and 2000, polycarbonate tubes, stainless steel tubes, or Oakfield probes were used to collect shallow (less than 6 in.) samples. Samples collected at depths greater than 6 in. generally were collected with a hand auger. Shallow samples (0-6 in.) generally were composites of 5 to 10 soil plugs collected from the sample area. Samples from depths greater than 6 in. were generally composites of three to five samples from the sample area.

Samples were chilled after collection and transported to the BOR Soils Laboratory in Denver, Colorado, where they were air dried, ground, sieved, and split. Samples were analyzed for total selenium by the NWQL and (or) the USGS Mineral Resources Laboratory in Denver, Colorado. The NWQL analyzed samples by digestion in hot nitric, hydrofluoric, and perchloric acids followed by hydride generation atomic absorption spectrophotometry (Fishman and Friedman, 1989). The USGS Mineral Resources Laboratory analyzed sediment samples by digestion in hydrochloric acid, hydrofluoric acid, perchloric acid, and aqua regia followed by atomic adsorption spectroscopy. A comparison of the results from each laboratory revealed no significant differences. Selenium speciation analyses were performed on bottom-material samples collected from Stewart Lake WMA in April 1996, April 2000, and August 2000. Selenium species measured in the April 1996 samples included soluble selenate ( $\mathrm{Se}(\mathrm{VI})$ ), adsorbed selenite (Se(IV)), elemental selenium (Se(0)), organic selenium, selenium oxides, and selenium in organic material separated from the sediment. These samples were analyzed at the University of Montana. Selenium species in the April and August 2000 samples were determined by a direct extraction procedure at the University of California, Riverside, Department of Environmental Sciences, followed by hydride generation atomic absorption spectroscopy (W.T. Frankenberger, Jr., and Y. Zhang, University of California, written commun., 2001). Selenium species determined by the direct extraction procedure included soluble selenate ( $\mathrm{Se}(\mathrm{VI})$ ), soluble selenite ( $\mathrm{Se}(\mathrm{IV})$ ), and soluble organic selenium (redox state of selenium unknown); $\mathrm{NaOH}$ extractable (insoluble) $\mathrm{Se}(\mathrm{VI})$, 
$\mathrm{Se}(\mathrm{IV})$, and organic selenium (redox state of selenium unknown); elemental selenium ( $\mathrm{Se}(0)$ ) plus selenide (Se(-II)); organic materials-related selenium (selenium in sediment organic materials after soluble and $\mathrm{NaOH}$ extractable organic selenium were removed from the samples); residue selenium; and total selenium. The total selenium determined by the direct extraction procedure differs from the total selenium determined at the USGS labs. The direct extraction procedure uses hydrochloric acid and hydrogen peroxide for total selenium digestion, rather than a series of strong acids used in the USGS digestions.

Suspended-sediment samples were either depth integrated using a DH-48TM sampler or discrete grab samples. The samples were processed and analyzed by the BOR soils laboratory in Denver, Colorado.

Sediment samples collected from Green River backwaters by the USFWS were collected with a stainless steel pan and scoop. Each sample was a composite of five subsamples. The samples were collected from the top $1 / 8$ in. of sediment. Roots and vegetation were carefully removed from the samples by hand. Samples were placed in plastic bags and chilled. The samples were analyzed by the Geochemical and Environmental Research Group (GERG) at Texas A\&M University.

\section{Biological Samples}

\section{Plants}

Aquatic plants were collected at most sites. Sampling schedules varied by site and by year. Species collected included those commonly available and those thought to be important as food or cover for aquatic birds. As many as three species were collected at each site. Generally, the non-rooted sections were collected, except for cattail and hardstem bullrush. The cattail samples typically consisted of 6 to 8 in. of stem starting at the root and 6 to 8 in. of root. Samples were double bagged in plastic and then frozen for storage. Samples were analyzed for inorganic elements only.

Birds

Few waterfowl were collected during the data collection period. Birds collected were shot with steel shot. Tissues analyzed were not contaminated by lead shot. Livers and whole-body juvenile birds were collected. All samples were placed in chemically clean jars or wrapped in aluminum foil for analyses. Data on age, sex, and weight were recorded.

The volume of each egg was measured by egg displacement in water; length by calipers to 0.1 millimeter (mm); net weight of egg contents to 1 gram. Eggs were usually opened by cracking the air-cell end with clean forceps and peeling the shell until the contents could be removed. Samples were placed in chemically clean jars and frozen. Eggs were analyzed individually.

Fish

Fish were collected predominantly by using trammel nets at Stewart Lake. In some instances, fish at Stewart Lake were collected with Fyke nets, dip nets, or minnow traps. Fish from the Green River were predominantly captured by electro-fishing. In most cases, each sample consisted of five adult fish of similar size. All adult fish were weighed, measured for total length, and double bagged in plastic bags prior to shipment for analysis. Three or more composite samples were collected at most data collection sites. In some samples consisting of small fish, only the weight of the composite sample was recorded.

Muscle plugs were opportunistically collected from endangered fish that were captured during routine sampling. Muscle plugs were collected with a sterile 4$\mathrm{mm}$ biopsy punch that was inserted into the fish with a twisting motion in the dorsal-medial quadrant of the fish. The plug was removed from the biopsy punch by being blown into a clean vial, placed in a sealable plastic bag, and stored on wet ice (less than 8 hours) or dry ice until it could be frozen. The hole in the fish was completely filled with an antibiotic ointment to speed healing. Measurements of total length, weight, body condition, and location also were collected for each endangered fish, except in the event of equipment failure. Endangered fish were immediately released after the muscle plug and field data were collected.

Invertebrates

Plankton samples were obtained by using light traps or plankton tow nets. All nonsnail invertebrates collected in the traps were included in these composite samples. Excess water was removed from samples, and samples were placed in chemically clean jars. Other invertebrates, such as snails and damselfly larvae, were opportunistically sampled by using Kick nets. 
Tissue samples were analyzed at a number of laboratories under contract to the USFWS, Patuxent Analytical Control Facility. Concentrations of the following elements were determined in the analyses of biological tissues: aluminum, arsenic, barium, beryllium, boron, cadmium, chromium, copper, iron, lead, magnesium, manganese, mercury, molybdenum, nickel, selenium, strontium, vanadium, and zinc. Muscle plugs from endangered fish were analyzed for selenium concentrations using neutron activation at the Columbia Environmental Research Center in Columbia, Missouri.

The reporting limits for constituents in biological samples varied over time and among laboratories performing the analyses. Reporting levels can be affected by a number of factors, including quantity of samples submitted, percent moisture, type of equipment used to analyze samples, and method of sampling.

\section{REFERENCES CITED}

Fishman, M.J., and Friedman, L.C., 1989, Methods for determination of inorganic substances in water and fluvial sediments: U.S. Geological Survey Techniques of Water-Resources Investigations, book 5, chap. A1, $709 \mathrm{p}$.

Peltz, L.A., and Waddell, Bruce, 1991, Physical, chemical, and biological data for detailed study of irrigation drainage in the middle Green River basin, Utah, 198889, with selected data for 1982-87: U.S. Geological Survey Open-File Report 91-530, 213 p.

Stephens, D.W., 1992, Selenium contamination of waterfowl areas in Utah and options for management, p. 301-311, in Robarts, R.D., and Bothwell, M.L., eds., Aquatic ecosystems in semi-arid regions-Implications for resource management, National Hydrology Research Institute Symposium Series 7, Environment Canada, Saskatoon.
Stephens, D.W., and Waddell, Bruce, 1998, Selenium sources and effects on biota in the Green River basin of Wyoming, Colorado, and Utah, p. 183-203 in

Frankenberger, W.T., Jr., and Engberg, R.A., eds., Environmental Chemistry of Selenium: Marcel Dekker, Inc., New York.

Stephens, D.W., Waddell, Bruce, and Miller, J.B., 1988, Reconnaissance investigation of water quality, bottom sediment, and biota associated with the irrigation drainage in the middle Green River basin, Utah, 198687: U.S. Geological Survey Water-Resources Investigations Report 88-4011, 70 p.

1991, Sources, sinks, and effects of selenium at Stewart Lake Waterfowl Management Area, Jensen, Utah, p. 324-329, in Mallard, G.E., and Aronson, D.A., eds., U.S. Geological Survey Toxic Substances Hydrology Program-Proceedings of the Technical Meeting, Monterey, California, March 11-15, 1991: U.S. Geological Survey Water-Resources Investigations Report 91-4034, 730 p.

Stephens, D.W., Waddell, Bruce, Peltz, L.A., and Miller, J.B., 1992, Detailed study of selenium and selected elements in water, bottom sediment, and biota associated with irrigation drainage in the middle Green River basin, Utah, 1988-90: U.S. Geological Survey Water-Resources Investigations Report 92-4084, 164 p.

U.S. Department of the Interior, 1997, Middle Green River Basin Study, Stewart Lake Waterfowl Management Area, Final Environmental Assessment: National Irrigation Water Quality Program, 113 p. 2002, National Irrigation Water Quality Program: Program Description, accessed June 25, 2002, at URL http://www.usbr.gov/niwqp/prgmdscr.html

U.S. Geological Survey, 1977, National handbook of recommended methods for water-data acquisition: Reston, Virginia, 12 numbered sections.

Wilde, R.D., and Radtke, D.B., eds., 1999, Collection of water samples, in National Field Manual for the Collection of Water-Quality Data: U.S. Geological Survey Techniques of Water-Resources Investigations, book 9, chap. A4, 130 p. 
Table 1. Hydrologic sampling-site designations, Bureau of Reclamation identifier, U.S. Geological Survey site identification number, and site type for data-collection sites in the middle Green River basin, Utah and Colorado

[USGS: U.S. Geological Survey; Site type: SW, surface water; LK, pond, lake, or reservoir; SP, spring or seep; GW, ground-water well]

\begin{tabular}{|c|c|c|c|c|}
\hline Site designation & $\begin{array}{c}\text { Short } \\
\text { site name }\end{array}$ & $\begin{array}{c}\text { Bureau of } \\
\text { Reclamation }\end{array}$ & USGS site ID number & Site type \\
\hline \multicolumn{5}{|c|}{ Green River drainage and selected tributaries (fig. 1) } \\
\hline Green River near Greendale, Utah & & & 09234500 & SW \\
\hline Yampa River near Maybell, Colorado & & & 09251000 & SW \\
\hline Green River near Jensen, Utah & & & 09261000 & SW \\
\hline Ashley Creek near Jensen, Utah & $\mathrm{AC} 40$ & GACO 30 & 09271500 & SW \\
\hline Ashley Creek below Union Canal diversion near Jensen, Utah & AC6750 & & 09271550 & SW \\
\hline Stewart Lake outflow near Jensen, Utah & & & 09271600 & LK \\
\hline Green River at Bonanza Bridge, near Vernal, Utah & & & 401840109283101 & SW \\
\hline Duchesne River near Randlett, Utah & & & 09302000 & SW \\
\hline White River near Watson, Utah & & & 09306500 & SW \\
\hline Pariette Draw at mouth near Ouray, Utah & & & 09307300 & SW \\
\hline Price River at Woodside, Utah & & & 09314500 & SW \\
\hline Green River at Green River, Utah & & & 09315000 & SW \\
\hline \multicolumn{5}{|c|}{ Brush Creek drainage (fig. 2) } \\
\hline Big Brush Creek above Red Fleet Reservoir, near Vernal, Utah & & & 09261700 & SW \\
\hline Red Fleet Reservoir inflow arm & RFR In & & 403451109260001 & LK \\
\hline Red Fleet Reservoir midlake & RFR Mid & & 403444109254001 & LK \\
\hline Red Fleet Reservoir near dam & RFR Dam & & 403428109253301 & LK \\
\hline Big Brush Creek below Red Fleet Reservoir & $\mathrm{BC} 1$ & & 403430109251801 & SW \\
\hline Big Brush Creek at county road near Donkey Flat & $\mathrm{BC} 2$ & & 403345109243501 & SW \\
\hline Brush Creek below Red Fleet at the corral & BC3 & & 403206109233501 & SW \\
\hline Brush Creek upstream of South Fork of Jensen Wash & $\mathrm{BC} 4$ & & 402940109230201 & SW \\
\hline Brush Creek at Sunshine Pipeline Diversion & BC5 & & 402840109233201 & SW \\
\hline Brush Creek at county road east of Bullwinkle Reservoir & BC6 & & 402756109235701 & SW \\
\hline Brush Creek at old diversion for Sunshine Canal & BC 7 & & 402623109235701 & SW \\
\hline Brush Creek at Burns Bench Canal diversion structure & $\mathrm{BC}$ BBC & & 402511109221501 & SW \\
\hline Burns Bench Canal below Sunshine Pipeline crossing & BBC & & 402426109204901 & SW \\
\hline NW 1 Seep to Burns Bench Canal, north of U.S. 40 & NW1 & & 402208109222601 & SP \\
\hline NW 2 Seep to Burns Bench Canal, north of U.S. 40 & NW2 & & 402208109222602 & SP \\
\hline NW 3 Seep to Burns Bench Canal, north of U.S. 40 & NW3 & & 402213109222101 & SP \\
\hline Burns Bench Canal at U.S. 40 & BBC 40 & & 402211109222601 & SW \\
\hline SW 1 Seep to Burns Bench Canal, south of U.S. 40 & SW1 & & 402208109222603 & SP \\
\hline Burns Bench Canal at diversion dam, south of U.S.40 & BBC Dam & & 402147109224101 & SW \\
\hline Pipeline from Burns Bench Canal to Stewart Lake & BBC Pipe & & 402134109214401 & SW \\
\hline Brush Creek near Jensen, Utah & $\mathrm{BC}$ & & 09263500 & SW \\
\hline \multicolumn{5}{|c|}{ Stewart Lake area (fig. 4) } \\
\hline Stewart Lake Inlet & SLI & & 402118109204801 & SW \\
\hline Stewart Lake Outlet & SLO & & 09271600 & SW \\
\hline J1 drain, Stewart Lake inflow & $\mathrm{J} 1$ & GASL01 & 402136109204103 & SW \\
\hline J1A drain, Stewart Lake inflow & $\mathrm{J} 1 \mathrm{~A}$ & & 402136109204104 & SW \\
\hline $\mathrm{J} 1$ and J1A drains (combined) & $\mathrm{J} 1 / \mathrm{J} 1 \mathrm{~A}$ & & 402136109204102 & SW \\
\hline J2 drain, Stewart Lake inflow & $\mathrm{J} 2$ & GASL02 & 402146109220301 & SW \\
\hline J3 drain, Stewart Lake inflow & $\mathrm{J} 3$ & GASL03 & 402134109221001 & SW \\
\hline J4 drain, Stewart Lake inflow & $\mathrm{J} 4$ & GASL04 & 402120109221901 & SW \\
\hline J2, J3, J4 drains (combined) & $\mathrm{J} 2, \mathrm{~J} 3, \mathrm{~J} 4$ & & 402037109215003 & SW \\
\hline North Seepage Collector at Stewart Lake & NorthCD & & 402134109215400 & SW \\
\hline Bureau of Reclamation Well E1 & E1 & & 402126109204901 & GW \\
\hline
\end{tabular}


Table 1. Hydrologic sampling-site designations, Bureau of Reclamation identifier, U.S. Geological Survey site identification number, and site type for data-collection sites in the middle Green River basin, Utah and Colorado-Continued

\begin{tabular}{|c|c|c|c|c|}
\hline Site designation & $\begin{array}{c}\text { Short } \\
\text { site name }\end{array}$ & $\begin{array}{c}\text { Bureau of } \\
\text { Reclamation }\end{array}$ & USGS site ID number & Site type \\
\hline \multicolumn{5}{|c|}{ Stewart Lake area (fig. 4)—Continued } \\
\hline Bureau of Reclamation Well E2 & E2 & & 402117109204901 & GW \\
\hline Bureau of Reclamation Well E3 & E3 & & 402111109210701 & GW \\
\hline Bureau of Reclamation Well W1 & W1 & & 402111109215901 & GW \\
\hline Bureau of Reclamation Well W2 & W2 & & 402106109220601 & GW \\
\hline Bureau of Reclamation Well W3 & W3 & & 402056109220301 & GW \\
\hline Seep 1 & S1 & & 402134109215301 & SW \\
\hline Seep 2 & S2 & & 402134109214301 & SW \\
\hline Seep 3 & S3 & & 402130109210001 & SW \\
\hline Seep 5 & S5 & & 402126109211901 & SW \\
\hline Seep 6 & S6 & & 402123109211301 & SW \\
\hline Seep 7 & S7 & & 402126109210501 & SW \\
\hline Seep 9 & S9 & & 402130109205702 & SW \\
\hline Seep 10 & S10 & & 402122109211302 & SW \\
\hline
\end{tabular}

Ashley Creek drainage,

Ashley Creek, and selected tributaries (fig. 3)

\begin{tabular}{|c|c|c|c|c|}
\hline Ashley Creek at 500 North, near Steinaker Draw & AC 500 & & 402749109295401 & SW \\
\hline Unnamed Tributary (east bank), below Sewer Lagoon & AT SL & & 402517109271001 & SW \\
\hline Ashley Central Canal return flow, at Ashley Creek & $\mathrm{ACC} \mathrm{AC}$ & & 402459109270401 & SW \\
\hline 4930 Tributary (west bank) above Sadlier Draw & AT 4930 & & 402432109262801 & SW \\
\hline Sadlier Draw at mouth-Sunshine/Burns Bench runoff & SD Mouth & & 402406109253401 & SW \\
\hline Ashley Creek at 6550 East, near Naples & AC 6550 & & 402354109254301 & SW \\
\hline Unnamed Tributary 14 (west bank), North of HWY 40 & AT14 & & 402347109254201 & SW \\
\hline Unnamed Tributary 12 (east bank), North of HWY 40 & AT 12 & & 402340109252701 & SW \\
\hline Unnamed Tributary 4 (east bank), North of HWY 40 & AT4 & & 402256109242901 & SW \\
\hline Unnamed Tributary 3 (east bank), North of HWY 40 & AT3 & & 402256109242501 & SW \\
\hline Mantle Gulch at mouth, inflow to Ashley Creek & MG Mouth & & 402241109243501 & SW \\
\hline Ashley Creek near Jensen & $\mathrm{AC} 40$ & GACO 30 & 09271500 & SW \\
\hline Ashley Creek below Union Canal Diversion near Jensen & AC 6750 & & 09271550 & SW \\
\hline Ashley Creek at mouth at the Green River & AC Mouth & & 402030109215401 & SW \\
\hline
\end{tabular}

Ashley Creek drainage,

Vernal Sewage Lagoons, and Winter Storage Pond (fig. 3)

\begin{tabular}{|c|c|c|c|}
\hline North Sewage Lagoon, Pond 1, at gate & NSL & 402550109270001 & LK \\
\hline Seep W31 at Winter Storage Pond, near Vernal & W31 & 402550109261701 & SW \\
\hline Seep W21 at Winter Storage Pond, near Vernal & W21 & 402540109261101 & SW \\
\hline Seep W7 at Winter Storage Pond, near Vernal & W7 & 402538109260502 & SW \\
\hline Seep W11 at Winter Storage Pond, near Vernal & W11 & 402538109260501 & SW \\
\hline Seep E1, East Arm of Winter Storage Pond, near Vernal & E1 & 402538109261701 & SW \\
\hline Wildlife Pond west of Ashley Sewage Lagoons, east inflow & WPE & 402534109265602 & SW \\
\hline Wildlife Pond west of Ashley Sewage Lagoons, north inflow & WPN & 402534109265603 & SW \\
\hline Seep W5 at Winter Storage Pond, near Vernal & W5 & 402535109260501 & SW \\
\hline Seep W1 at Winter Storage Pond, near Vernal & $\mathrm{W} 1$ & 402530109261101 & SW \\
\hline Seep W0 at Winter Storage Pond, near Vernal & W0 & 402519109260101 & SW \\
\hline New state permit site for Ashley Sewage Lagoons & NSP & 402519109265700 & SW \\
\hline North Canal near Ashley Sewage Lagoons, near Vernal & $\mathrm{NC}$ & 402516109270301 & SW \\
\hline Abandoned farm drain, upper end, near Ashley Sewage Lagoons & AFD & 402516109270302 & SW \\
\hline Winter Storage Pond & WSP & 402516109260001 & LK \\
\hline Winter Storage Pond at outflow Structure & WSP Out & 402510109255301 & SW \\
\hline
\end{tabular}


Table 1. Hydrologic sampling-site designations, Bureau of Reclamation identifier, U.S. Geological Survey site identification number, and site type for data-collection sites in the middle Green River basin, Utah and Colorado-Continued

\begin{tabular}{|c|c|c|c|c|}
\hline Site designation & $\begin{array}{l}\text { Short } \\
\text { site name }\end{array}$ & $\begin{array}{l}\text { Bureau of } \\
\text { Reclamation }\end{array}$ & USGS site ID number & Site type \\
\hline \multicolumn{5}{|c|}{$\begin{array}{c}\text { Ashley Creek drainage, } \\
\text { canals, ponds, and drains (fig. 3) }\end{array}$} \\
\hline Rock Point Canal at 1500 West in Vernal & RPC 1500 & & 402918109295801 & SW \\
\hline 17A2 Drain, near Vernal & $17 \mathrm{~A} 2$ & $17 \mathrm{~A} 2$ & 402830109343301 & SW \\
\hline 17B1 Drain, near Vernal & 17B1 & 17B1 & 402804109345301 & SW \\
\hline 17B2 Drain, near Vernal & 17B2 & 17B2 & 402802109350001 & SW \\
\hline 17A1 Drain, near Vernal & $17 \mathrm{~A} 1$ & $17 \mathrm{~A} 1$ & 402802109343901 & SW \\
\hline 16A1 Drain, Vernal & $16 \mathrm{~A} 1$ & $16 \mathrm{~A} 1$ & 402802109340901 & SW \\
\hline Steinaker Service Canal at 500 North in Vernal & SSC 500 & & 402746109332701 & SW \\
\hline 20A2 Drain, Vernal & $20 \mathrm{~A} 2$ & $20 \mathrm{~A} 2$ & 402736109352301 & SW \\
\hline Amos/Merkley Drain in Maeser & $\mathrm{AM}$ & $\mathrm{AM}$ & 402720109333401 & SW \\
\hline 22A1 Drain near Vernal & $22 \mathrm{~A} 1$ & $22 \mathrm{~A} 1$ & 402653109331301 & SW \\
\hline 27A2 Drain, Vernal & $27 \mathrm{~A} 2$ & $27 \mathrm{~A} 2$ & 402651109330401 & SW \\
\hline 27C2 Drain, Vernal & $27 \mathrm{C} 2$ & $27 \mathrm{C} 2$ & 402644109321901 & SW \\
\hline 26A2 Drain, Vernal & $26 \mathrm{~A} 2$ & $26 \mathrm{~A} 2$ & 402644109321101 & SW \\
\hline 26B2 Drain, near Vernal & 26B2 & $26 \mathrm{~B} 2$ & 402636109314101 & SW \\
\hline 30B1 Drain, near Naples & 30B1 & 30B1 & 402634109291501 & SW \\
\hline 26C1 Drain, Vernal & $26 \mathrm{C} 1$ & $26 \mathrm{C} 1$ & 402633109313501 & SW \\
\hline 26C2 Drain, near Vernal & $26 \mathrm{C} 2$ & $26 \mathrm{C} 2$ & 402625109311501 & SW \\
\hline 25A2 Drain, Vernal & $25 \mathrm{~A} 2$ & $25 \mathrm{~A} 2$ & 402624109310001 & SW \\
\hline Pond 30A, in Naples & $30 \mathrm{~A}$ & $30 \mathrm{~A}$ & 402617109294901 & LK \\
\hline 29A1 Drain, near Naples & 29A1 & 29A1 & 402611109282901 & SW \\
\hline Open Pilot Drain, near Vernal & OPD & OPD & 402555109303301 & SW \\
\hline Pilot Drain, near Vernal & $\mathrm{PD}$ & PD & 402555109301301 & SW \\
\hline Pond $31 \mathrm{~A} 1$ at 1830 East 1700 South, in Naples & P31A1 & P31A1 & 402555109293202 & LK \\
\hline 31A1 Drain, Vernal & $31 \mathrm{~A} 1$ & $31 \mathrm{~A} 1$ & 402555109293201 & SW \\
\hline 32A1 Drain, near Naples & $32 \mathrm{~A} 1$ & $32 \mathrm{~A} 1$ & 402553109282301 & SW \\
\hline Ashley Central Canal at flume, 2500 South, Naples & ACC 2500 & & 402511109293201 & SW \\
\hline Steinaker Service Canal at 2500 South near 500 West & SSC 2500 & & 402509109322301 & SW \\
\hline 5A1 Drain, near Naples & $5 \mathrm{~A} 1$ & $5 \mathrm{~A} 1$ & 402506109283501 & SW \\
\hline Pond on Ashley Central Canal at 2500 South St., Naples & PACC & & 402505109281401 & LK \\
\hline Ashley Central Canal return flow, at Ashley Creek & $\mathrm{ACC} A C$ & & 402459109270401 & SW \\
\hline Steinaker Service Canal at 4500 South, in Vernal & SSC 4500 & & 402324109310201 & SW \\
\hline River Irrigation Canal at diversion, 6800 South in Naples & RIC 6800 & & 402305109241901 & SW \\
\hline Jackson Farm Pond at 5000 South St., near Naples & JFP 5000 & & 402258109291001 & LK \\
\hline Mantle Gulch at mouth, inflow to Ashley Creek & MG Mouth & & 402241109243501 & SW \\
\hline Highline Canal near Asphalt Ridge near Vernal & $\mathrm{HC}$ & & 402146109300301 & SW \\
\hline Squires Farm Pond on Steinaker Service Canal & SFP & & 402114109274701 & LK \\
\hline Steinaker Service Canal at Mantle Gulch near Vernal & SSC MG & & 402214109295101 & SW \\
\hline Upper Mantle Gulch at U.S. 45, road to Bonanza & UMG 45 & & 402222109295101 & SW \\
\hline Mantle Gulch at Oilfield Road East, Naples & MG OF & & 402233109251601 & SW \\
\hline
\end{tabular}


Table 2. Site number and site ID for bottom-sediment and suspended-sediment-samplng sites sampled by the Bureau of Reclamation, the U.S. Fish and Wildlife Service, and the U.S. Geological Survey at and near Stewart Lake Waterfowl Management Area, middle Green River basin, eastern Utah

\begin{tabular}{|c|c|c|}
\hline Site ID number & Permanent site ID & Comments \\
\hline \multicolumn{3}{|r|}{ Bureau of Reclamation sediment-sampling sites (fig. 5) } \\
\hline $2048-2148$ & $\mathrm{~S} 1$ & Undisturbed sample site \\
\hline 2048-2148 & S1 Till plot & 100-by-100-foot plot that is tilled to a depth of 6 inches \\
\hline $2048-2148$ & S1 Box plot & 8 -by-8-foot by 6 -inch plot in the till plot that is lined with plastic \\
\hline 2048-2148 & S1 Control plot & 100-by-100-foot plot adjacent to the till plot that is undisturbed \\
\hline $2049-2157$ & & Undisturbed sample site \\
\hline $2051-2131$ & & Undisturbed sample site \\
\hline 2053-2143 & $\mathrm{S} 2$ & Undisturbed sample site \\
\hline $2055-2118$ & & Undisturbed sample site \\
\hline $2055-2154$ & S3 & Undisturbed sample site \\
\hline $2056-2144$ & $\mathrm{~S} 4$ & Undisturbed sample site \\
\hline $2057-2201$ & S5 & Undisturbed sample site \\
\hline $2100-2127$ & S6 & Undisturbed sample site \\
\hline $2101-2218$ & & Undisturbed sample site \\
\hline $2102-2210$ & & Undisturbed sample site \\
\hline $2105-2122$ & S7 & Undisturbed sample site \\
\hline $2105-2145$ & S8 & Undisturbed sample site \\
\hline $2108-2225$ & & Undisturbed sample site \\
\hline $2110-2105$ & & Undisturbed sample site \\
\hline $2110-2130$ & S9 & Undisturbed sample site \\
\hline $2110-2202$ & & Undisturbed sample site \\
\hline $2110-2202$ & & Undisturbed sample site \\
\hline $2112-2100$ & & Undisturbed sample site \\
\hline $2112-2158$ & & Undisturbed sample site \\
\hline $2112-2212$ & & Undisturbed sample site \\
\hline $2113-2119$ & S10 & Undisturbed sample site \\
\hline $2113-2119$ & S10 Till plot & 100-by-100-foot plot that is tilled to a depth of 6 inches \\
\hline 2113-2119 & S10 Box plot & 8 -by-8-foot by 6 -inch plot in the till plot that is lined with plastic \\
\hline $2113-2119$ & S10 Control plot & 100-by-100-foot plot adjacent to the till plot that is undisturbed \\
\hline 2114-2211 & & Undisturbed sample site \\
\hline $2116-2150$ & S11 & Undisturbed sample site \\
\hline $2117-2108$ & & Undisturbed sample site \\
\hline $2117-2132$ & $\mathrm{~S} 12$ & Undisturbed sample site \\
\hline $2118-2105$ & S13 & Undisturbed sample site \\
\hline $2119-2158$ & & Undisturbed sample site \\
\hline $2120-2136$ & & Undisturbed sample site \\
\hline $2121-2120$ & & Undisturbed sample site \\
\hline $2121-2136$ & S14 & Undisturbed sample site \\
\hline $2123-2111$ & & Undisturbed sample site \\
\hline $2123-2130$ & & Undisturbed sample site \\
\hline $2123-2144$ & S15 & Undisturbed sample site \\
\hline $2123-2144$ & S15 Till plot & 100-by-100-foot plot that is tilled to a depth of 6 inches \\
\hline $2123-2144$ & S15 Box plot & 8-by-8-foot by 6 -inch plot in the till plot that is lined with plastic \\
\hline $2123-2144$ & S15 Control plot & 100 -by-100-foot plot adjacent to the till plot that is undisturbed \\
\hline 2124-2149 & & Undisturbed sample site \\
\hline 2124-2203 & & Undisturbed sample site \\
\hline
\end{tabular}


Table 2. Site number and site ID for bottom-sediment and suspended-sediment-samplng sites sampled by the Bureau of Reclamation, the U.S. Fish and Wildlife Service, and the U.S. Geological Survey at and near Stewart Lake Waterfowl Management Area, middle Green River basin, eastern UtahContinued

\begin{tabular}{|c|c|c|}
\hline Site ID number & Permanent site ID & Comments \\
\hline \multicolumn{3}{|c|}{ Bureau of Reclamation sediment-sampling sites (fig. 5)—Continued } \\
\hline $2125-2110$ & S16 & Undisturbed sample site \\
\hline $2125-2113$ & & Undisturbed sample site \\
\hline $2126-2100$ & S17 & Undisturbed sample site \\
\hline $2126-2136$ & S18 & Undisturbed sample site \\
\hline $2127-2159$ & & Undisturbed sample site \\
\hline 2129-2059 & & Undisturbed sample site \\
\hline $2129-2203$ & & Undisturbed sample site \\
\hline $2130-2146$ & S19 & Undisturbed sample site \\
\hline $2131-2159$ & & Undisturbed sample site \\
\hline $2132-2144$ & $\mathrm{~S} 20$ & Undisturbed sample site \\
\hline $2134-2158$ & & Undisturbed sample site \\
\hline $2135-2153$ & & Undisturbed sample site \\
\hline $2138-2043$ & & Undisturbed sample site \\
\hline B2 & & Undisturbed sample site \\
\hline B3 & & Undisturbed sample site \\
\hline B5 & & Undisturbed sample site \\
\hline B7 & & Undisturbed sample site \\
\hline B8 & & Undisturbed sample site \\
\hline B6 & & Undisturbed sample site \\
\hline $\mathrm{J} 3$ & & Undisturbed sample site \\
\hline $\mathrm{J} 4$ & & Undisturbed sample site \\
\hline
\end{tabular}

U.S. Fish and Wildlife Service sediment-sampling sites (fig. 9)

\begin{tabular}{ll}
\hline GR1 & Green River backwater near Stewart Lake \\
GR2 & Green River backwater near Stewart Lake \\
GR3 & Green River backwater near Stewart Lake \\
GR4 & Green River backwater near Stewart Lake \\
GR5 & Green River backwater near Stewart Lake \\
GR6 & Green River backwater near Stewart Lake \\
J1B & Green River backwater near JI drain outfall \\
\hline
\end{tabular}

U.S. Geological Survey and Bureau of Reclamation sediment and suspended-sediment-sampling sites (figs. 6, 7, and 8)

\begin{tabular}{ll}
\hline J1 0 & Sediment-sampling site, drain J1 area \\
J1 700 & Sediment-sampling site, drain J1 area \\
J1 900 & Sediment-sampling site, drain J1 area \\
J1 1200 & Sediment-sampling site, drain J1 area \\
J1 1700 & Sediment-sampling site, drain J1 area \\
J1 2000 & Sediment-sampling site, drain J1 area \\
J1 2200 & Sediment-sampling site, drain J1 area \\
J2 Pond & Sediment-sampling site, drain J2 area \\
J2 100 & Sediment-sampling site, drain J2 area \\
J3-30 & Sediment-sampling site, drain J3 area \\
J3-60 & Sediment-sampling site, drain J3 area \\
J3-90 & Sediment-sampling site, drain J3 area
\end{tabular}


Table 2. Site number and site ID for bottom-sediment and suspended-sediment-samplng sites sampled by the Bureau of Reclamation, the U.S. Fish and Wildlife Service, and the U.S. Geological Survey at and near Stewart Lake Waterfowl Management Area, middle Green River basin, eastern Utah— Continued

\begin{tabular}{|c|c|c|}
\hline Site ID number & Permanent site ID & Comments \\
\hline \multicolumn{3}{|c|}{ U.S. Geological Survey and Bureau of Reclamation sediment and suspended-sediment-sampling sites (figs. 6, 7, and 8)—Continued } \\
\hline $\mathrm{J} 3-150$ & & Sediment-sampling site, drain J3 area \\
\hline $\mathrm{J} 3-210$ & & Sediment-sampling site, drain J3 area \\
\hline $\mathrm{J} 3-270$ & & Sediment-sampling site, drain J3 area \\
\hline $\mathrm{J} 3-330$ & & Sediment-sampling site, drain J3 area \\
\hline J3-390 & & Sediment-sampling site, drain J3 area \\
\hline $\mathrm{J} 4-10$ & & Sediment-sampling site, drain J4 area \\
\hline J4-20 & & Sediment-sampling site, drain J4 area \\
\hline $\mathrm{J} 4-30$ & & Sediment-sampling site, drain J4 area \\
\hline J4-60 & & Sediment-sampling site, drain J4 area \\
\hline $\mathrm{J} 4-75$ & & Sediment-sampling site, drain J4 area \\
\hline J4-90 & & Sediment-sampling site, drain J4 area \\
\hline J4 Pond 0 & & Sediment-sampling site, drain J4 area \\
\hline J4-100 Right & & Sediment-sampling site, drain J4 area \\
\hline J4-100 Left & & Sediment-sampling site, drain J4 area \\
\hline $\mathrm{J} 4-150$ & & Sediment-sampling site, drain J4 area \\
\hline J4-Pond 200 & & Sediment-sampling site, drain J4 area \\
\hline J4-200 & & Sediment-sampling site, drain J4 area \\
\hline $\mathrm{J} 4-210$ & & Sediment-sampling site, drain J4 area \\
\hline $\mathrm{J} 4-270$ & & Sediment-sampling site, drain J4 area \\
\hline $\mathrm{J} 4-330$ & & Sediment-sampling site, drain J4 area \\
\hline J4-400W & & Sediment-sampling site, drain J4 area \\
\hline $\mathrm{J} 4-400 \mathrm{E}$ & & Sediment-sampling site, drain J4 area \\
\hline J4-600W & & Sediment-sampling site, drain J4 area \\
\hline J4-600E & & Sediment-sampling site, drain J4 area \\
\hline $\mathrm{J} 4-800 \mathrm{E}$ & & Sediment-sampling site, drain J4 area \\
\hline $\mathrm{J} 4-1600$ & & Sediment-sampling site, drain J4 area \\
\hline J4-1830-A & & Sediment-sampling site, drain J4 area \\
\hline J4-1830-B & & Sediment-sampling site, drain J4 area \\
\hline J4-1830-C & & Sediment-sampling site, drain J4 area \\
\hline J4-2150-A & & Sediment-sampling site, drain J4 area \\
\hline J4-2150-B & & Sediment-sampling site, drain J4 area \\
\hline $\mathrm{J} 4-2150-\mathrm{C}$ & & Sediment-sampling site, drain $\mathrm{J} 4$ area \\
\hline J4-2230-A & & Sediment-sampling site, drain J4 area \\
\hline J4-2230-B & & Sediment-sampling site, drain J4 area \\
\hline $\mathrm{J} 4-2230-\mathrm{C}$ & & Sediment-sampling site, drain J4 area \\
\hline J4 2600 & & Sediment-sampling site, drain J4 area \\
\hline J4 3000 & & Sediment-sampling site, drain J4 area \\
\hline J4 3200 Seep & & Sediment-sampling site, drain J4 area \\
\hline J4 3200 & & Sediment-sampling site, drain J4 area \\
\hline J4 3500 & & Sediment-sampling site, drain J4 area \\
\hline $\mathrm{J} 4-3800$ & & Sediment-sampling site, drain J4 area \\
\hline J4 4600 & & Sediment-sampling site, drain J4 area \\
\hline SS1 & & Suspended-sediment sampling site \\
\hline SS2 & & Suspended-sediment sampling site \\
\hline
\end{tabular}


Table 2. Site number and site ID for bottom-sediment and suspended-sediment-samplng sites sampled by the Bureau of Reclamation, the U.S. Fish and Wildlife Service, and the U.S. Geological Survey at and near Stewart Lake Waterfowl Management Area, middle Green River basin, eastern UtahContinued

\section{Site ID number Permanent site ID Comments}

U.S. Geological Survey and Bureau of Reclamation sediment and suspended-sediment-sampling sites (figs. 6, 7, and 8)—Continued

SS3

SS4

SS5

SS6

SS7
Suspended-sediment sampling site

Suspended-sediment sampling site

Suspended-sediment sampling site

Suspended-sediment sampling site

Suspended-sediment sampling site 
Table 3. Site name and abbreviated site name for biological-sampling sites sampled by the U.S. Fish and Wildlife Service, middle Green River basin, eastern Utah

\begin{tabular}{|c|c|c|c|}
\hline Site name & $\begin{array}{l}\text { Abbreviated } \\
\text { site name }\end{array}$ & Site name & $\begin{array}{l}\text { Abbreviated } \\
\text { site name }\end{array}$ \\
\hline \multicolumn{2}{|l|}{ Ashley Creek Area (fig. 9) } & \multirow{2}{*}{\multicolumn{2}{|c|}{$\begin{array}{c}\text { Green River area near Stewart Lake Waterfowl } \\
\text { Management Area (fig. 9)—Continued }\end{array}$}} \\
\hline Ashley Creek at Bridge in Jensen & $\mathrm{ACBJ}$ & & \\
\hline Ashley Creek at Burns Bench Return Channel & ACBR & Backwater below Jensen Bridge & GR1 \\
\hline Ashley Creek at Confluence with Green River & ACGR & Backwater formed by the $\mathrm{J} 1 / \mathrm{J} 1 \mathrm{~A}$ Outfall & J1B \\
\hline Ashley Creek at HWY40 & AC40 & Bonanza & BO \\
\hline Ashley Creek at HWY40 & $\mathrm{AC} 40$ & Bonanza Bridge & BB \\
\hline Ashley Creek at Road to Vernal Sewer Lagoons & $\mathrm{ACV}$ & Collier Draw & $\mathrm{CD}$ \\
\hline Ashley Creek at Winter Storage Pond & WSP & Hamacker Bottom & HA \\
\hline Ashley Creek near Naples & $\mathrm{ACN}$ & Horseshoe Bend & HB \\
\hline Ashley Creek near Stewart Lake & ACS & Oxbow south of Stewart Lake & SLOX \\
\hline Ashley Valley Central Pond & AVCP & Stewart Lake Outlet Channel & SLOC \\
\hline Ashley Creek & SA & \multicolumn{2}{|c|}{ Ouray area (fig. 10) } \\
\hline Keith Squires Pond & KSP & Leota Bottom & LB \\
\hline Lane Jackson Pond & LJP & Leota 6, Ouray NWR & L6 \\
\hline Mantle Gulch & MG & North Roadside & NR \\
\hline \multirow{2}{*}{$\begin{array}{l}\text { Marsh on Murray Property at Ashley Creek at } \\
\text { HWY40 }\end{array}$} & MMM & Old Charlie Wash & OCW \\
\hline & & Ouray National Fish Hatchery & Ouray NFH \\
\hline Melvin Smith Residence & MSR & Sheppard Bottom & SB \\
\hline Pond 30A & $30 \mathrm{~A}$ & Sheppard 3, Ouray NWR & S3 \\
\hline Winter Storage Pond & WSP & Sheppard 5, Ouray NWR & S5 \\
\hline \multicolumn{2}{|l|}{ Brush Creek area (fig. 9) } & South Roadside, Ouray NWR & SR \\
\hline Brush Creek & $\mathrm{BC}$ & Woods Bottom & WB \\
\hline Brush Creek at Two Bridge & $\mathrm{BCD}$ & \multicolumn{2}{|c|}{ Pariette Wetlands area (fig. 10) } \\
\hline Brush Creek at HWY 149 & BC149 & Big Island Pond & BIP \\
\hline Escalante Bar & EB & Desiltation Pond & $\mathrm{DP}$ \\
\hline Escalante Marsh above Jensen & EM & Gadwall Pond & GWP \\
\hline \multirow{2}{*}{\multicolumn{2}{|c|}{$\begin{array}{l}\text { Green River area near Dinosaur } \\
\text { National Monument (fig. 9) }\end{array}$}} & Stewart Lake area & \\
\hline & & Gravel Pit near Jensen & GPJ \\
\hline Boneyard & D 1 & Stewart Lake Drains J2-J4 & SLJ2-4 \\
\hline Split Mountain & SM & Stewart Lake Inlet & SLI \\
\hline Razorback Bar & RZB & Stewart Lake J1 Drain & SLJ1 \\
\hline $\begin{array}{l}\text { Green River area near Stewart I } \\
\text { Waterfowl Management Area }(f \mathrm{i}\end{array}$ & & Stewart Lake J2 Drain & SLJ2 \\
\hline & & Stewart Lake J4 Drain & SLJ4 \\
\hline Backwater above Bonanza Pump station & GR6 & Stewart Lake Middle & SLM \\
\hline Backwater above Red Wash Boat Launch & $\begin{array}{ll}\text { GR5 } \\
\text { GP3 }\end{array}$ & Stewart Lake North Overlook & SLN \\
\hline Backwater above Stewart Lake Outlet & GR3 & Stewart Lake Outlet & SLO \\
\hline Backwater at Stewart Lake Inlet & GR2 & Stewart Lake Southwest Dike & SSD \\
\hline Backwater below Ashley Creek & GR4 & Stewart Lake Frog Pond & SFP \\
\hline
\end{tabular}


Table 4. Physical properties and chemical analyses for quality-control samples collected during 1993-96 from the middle Green River basin, Utah and Colorado

$\left[\mu \mathrm{S} / \mathrm{cm}\right.$, microsiemens per centimeter at 25 degrees Celsius; ${ }^{\circ} \mathrm{C}$, degrees Celsius; mg/L, milligrams per liter; $\mu \mathrm{g} / \mathrm{L}, \mathrm{micrograms}$ per liter; - , no data; <, less than]

\begin{tabular}{|c|c|c|c|c|c|c|c|}
\hline Date & Time & $\begin{array}{c}\text { Equipment blank, type } \\
\text { of solution }\end{array}$ & $\begin{array}{c}\text { Specific } \\
\text { conductance, } \\
\text { laboratory } \\
(\mu \mathrm{S} / \mathrm{cm})\end{array}$ & $\begin{array}{l}\text { Solids, residue } \\
\text { at } 180^{\circ} \mathrm{C} \\
\text { dissolved } \\
(\mathrm{mg} / \mathrm{L})\end{array}$ & $\begin{array}{c}\text { Boron, } \\
\text { dissolved ( } \mu \mathrm{g} / \mathrm{L} \\
\text { as B) }\end{array}$ & $\begin{array}{c}\text { Selenium, } \\
\text { dissolved } \\
(\mu \mathrm{g} / \mathrm{L} \\
\text { as } \mathrm{Se})\end{array}$ & $\begin{array}{c}\text { Zinc, dissolved } \\
(\mu g / L \\
\text { as } Z n)\end{array}$ \\
\hline $03-26-93$ & 1045 & Deionized water & - & - & - & 32 & - \\
\hline $06-23-93$ & 1700 & Deionized water & - & - & - & $<1$ & - \\
\hline $07-27-93$ & 1205 & Deionized water & - & - & - & $<1$ & - \\
\hline $08-24-93$ & 1900 & Deionized water & - & - & - & $<1$ & - \\
\hline $08-25-93$ & 1545 & Deionized water & - & - & - & $<1$ & - \\
\hline $10-13-93$ & 1530 & Deionized water & 2 & 1 & $<10$ & $<1$ & $<10$ \\
\hline 04-14-94 & 1200 & Deionized water & - & - & - & $<1$ & - \\
\hline $05-19-94$ & 1025 & Deionized water & - & - & - & $<1$ & - \\
\hline $07-14-94$ & 1615 & Deionized water & - & - & - & $<1$ & - \\
\hline $08-17-94$ & 1400 & Deionized water & - & - & - & $<1$ & - \\
\hline $09-21-94$ & 1720 & Deionized water & - & - & - & $<1$ & - \\
\hline $03-21-95$ & 1700 & Deionized water & - & - & 40 & $<1$ & $<10$ \\
\hline $04-19-95$ & 1800 & Deionized water & - & - & 30 & $<1$ & $<10$ \\
\hline $05-18-95$ & 1730 & Deionized water & - & - & $<10$ & $<1$ & $<10$ \\
\hline $06-22-95$ & 1900 & Deionized water & - & - & - & $<1$ & $<10$ \\
\hline $07-18-95$ & 1900 & Deionized water & - & - & 30 & $<1$ & $<10$ \\
\hline $08-17-95$ & 1930 & Deionized Water & - & - & - & $<1$ & $<10$ \\
\hline $09-23-95$ & 1100 & Deionized Water & - & - & - & $<1$ & - \\
\hline $10-25-95$ & 1800 & Deionized Water & - & - & 30 & 2 & $<10$ \\
\hline $03-27-96$ & 1030 & Deionized Water & - & - & - & $<1$ & $<10$ \\
\hline $04-17-96$ & 1800 & Deionized Water & - & - & - & $<1$ & $<10$ \\
\hline $05-16-96$ & 1630 & Deionized Water & - & - & - & $<1$ & - \\
\hline $06-14-96$ & 0900 & Deionized Water & - & - & - & $<1$ & - \\
\hline $07-17-96$ & 1730 & Deionized Water & - & - & - & $<1$ & - \\
\hline $08-21-96$ & 1245 & Deionized Water & - & - & - & $<1$ & - \\
\hline $09-20-96$ & 0930 & Deionized Water & - & - & - & $<1$ & - \\
\hline $10-25-96$ & 1820 & Deionized Water & - & - & - & $<1$ & - \\
\hline
\end{tabular}


Table 5. Discharge, physical properties, and selenium concentration of water from the Green River and selected tributaries, Utah and Colorado, water years 1991-2000

[Data from U.S. Geological Survey; $\mathrm{ft}^{3} / \mathrm{s}$, cubic feet per second; ${ }^{\circ} \mathrm{C}$, degrees Celsius; $\mu \mathrm{S} / \mathrm{cm}$, microsiemens per centimeter at $25^{\circ} \mathrm{C} ; \mu \mathrm{g} / \mathrm{L}$, micrograms per liter; Number in parentheses is the U.S. Geological Survey Site ID number in table $1 ;<$, less than; —, no data; E, estimated]

\begin{tabular}{|c|c|c|c|c|c|c|c|}
\hline Date & Time & $\begin{array}{c}\text { Discharge, } \\
\text { instant- } \\
\text { aneous } \\
\left(\mathrm{ft}^{3} / \mathrm{s}\right)\end{array}$ & $\begin{array}{l}\text { Temper- } \\
\text { ature } \\
\left({ }^{\circ} \mathrm{C}\right)\end{array}$ & $\begin{array}{c}\text { Specific } \\
\text { conduc- } \\
\text { tance } \\
(\mu \mathrm{S} / \mathrm{cm})\end{array}$ & $\begin{array}{c}\mathrm{pH}, \text { water, } \\
\text { whole, field } \\
\text { (standard units) }\end{array}$ & $\begin{array}{c}\text { Selenium, } \\
\text { dissolved } \\
(\mu \mathrm{g} / \mathrm{L} \text { as Se) }\end{array}$ & $\begin{array}{c}\text { Selenium, } \\
\text { total } \\
(\mu \mathrm{g} / \mathrm{L} \text { as } \mathrm{Se})\end{array}$ \\
\hline \multicolumn{8}{|c|}{ Green River near Greendale, Utah $(09234500)$} \\
\hline $11-08-90$ & 1430 & 913 & 8.5 & 780 & 8.2 & $<1$ & - \\
\hline $12-13-90$ & 1230 & 884 & 5.5 & 810 & 7.6 & $<1$ & - \\
\hline $02-27-91$ & 1230 & 868 & 4.0 & 830 & 8.2 & 1.0 & - \\
\hline $04-24-91$ & 1230 & 883 & 7.0 & 790 & 8.3 & - & - \\
\hline $06-19-91$ & 1330 & 2,870 & 11.0 & 720 & 8.4 & - & - \\
\hline $08-29-91$ & 1000 & 1,760 & 11.5 & 760 & 8.2 & $<1$ & - \\
\hline $10-02-91$ & 1430 & 1,710 & 12.5 & 740 & 8.2 & - & - \\
\hline $12-04-91$ & 1240 & 860 & 6.0 & 790 & 7.8 & $<1$ & - \\
\hline $02-04-92$ & 1200 & 2,180 & 3.0 & 816 & 8.1 & $<1$ & - \\
\hline 04-09-92 & 1200 & 1,460 & 6.5 & 750 & 8.2 & 2.0 & - \\
\hline $06-02-92$ & 1230 & 861 & 11.5 & 710 & 8.5 & $<1$ & - \\
\hline 08-04-92 & 1300 & 1,300 & 12.5 & 710 & 8.3 & $<1$ & - \\
\hline $10-06-92$ & 1330 & 902 & 11.5 & 730 & 8.3 & $<1$ & - \\
\hline $12-08-92$ & 1530 & 881 & 4.5 & 750 & 8.4 & 1.0 & - \\
\hline 03-09-93 & 1500 & 910 & 3.0 & 770 & 8.3 & $<1$ & - \\
\hline $05-12-93$ & 1330 & 2,800 & 13.0 & 740 & 8.4 & 1.0 & - \\
\hline 07-13-93 & 1530 & 897 & 12.5 & 710 & 8.3 & $<1$ & - \\
\hline 09-14-93 & 1400 & 1,490 & 13.0 & 750 & 8.5 & 1.0 & - \\
\hline $11-04-93$ & 1300 & 2,980 & 10.0 & 770 & 8.3 & $<1$ & - \\
\hline 01-12-94 & 1430 & 1,760 & 4.0 & 810 & 8.1 & $<1$ & - \\
\hline $03-17-94$ & 1100 & 864 & 4.0 & 780 & 8.5 & $<1$ & - \\
\hline $05-20-94$ & 1000 & 4,700 & 9.0 & 740 & 8.5 & $<1$ & - \\
\hline 07-29-94 & 1515 & 1,670 & 13.0 & 742 & 8.5 & $<1$ & - \\
\hline $10-18-94$ & 1330 & 1,410 & 10.0 & 730 & 8.3 & $<1$ & - \\
\hline $11-29-94$ & 1055 & 1,170 & 7.0 & 750 & 8.3 & - & - \\
\hline $03-02-95$ & 1110 & 847 & 4.0 & 750 & 8.5 & $<1$ & - \\
\hline 04-05-95 & 1120 & 872 & 5.5 & 740 & 8.3 & 1.0 & - \\
\hline $05-16-95$ & 0950 & 1,920 & 7.0 & 750 & 8.3 & $<1$ & - \\
\hline 07-13-95 & 0955 & 3,160 & 12.5 & 630 & 8.3 & $<1$ & - \\
\hline $08-29-95$ & 0945 & 1,380 & 12.0 & 680 & 8.3 & $<1$ & - \\
\hline $10-04-95$ & 0905 & 1,390 & 13.0 & 690 & 8.3 & $<1$ & - \\
\hline $12-12-95$ & 1000 & 3,110 & 7.0 & 750 & 8.4 & - & - \\
\hline 04-03-96 & 0945 & 2,770 & 4.0 & 730 & 8.4 & $<1$ & - \\
\hline $05-16-96$ & 0945 & 4,610 & 7.5 & 700 & 8.4 & $<1$ & - \\
\hline $07-10-96$ & 0920 & 1,370 & 12.0 & 680 & 8.5 & $<1$ & - \\
\hline $08-21-96$ & 0930 & 1,640 & 12.0 & 700 & 8.3 & $<1$ & - \\
\hline $10-15-96$ & 1130 & 1,710 & 12.0 & 690 & 8.4 & $<1$ & - \\
\hline $12-04-96$ & 1200 & 2,040 & 7.0 & 740 & 8.2 & $<1$ & - \\
\hline $01-29-97$ & 0930 & 2,720 & 4.0 & 720 & 8.3 & $<1$ & - \\
\hline 03-06-97 & 1200 & 3,120 & 4.0 & 680 & 8.4 & $<1$ & - \\
\hline $04-17-97$ & 0930 & 4,500 & 4.5 & 680 & 8.4 & $<1$ & - \\
\hline $05-21-97$ & 0830 & 4,720 & 8.0 & 640 & 8.2 & $<1$ & - \\
\hline
\end{tabular}


Table 5. Discharge, physical properties, and selenium concentration of water from the Green River and selected tributaries, Utah and Colorado, water years 1991-2000-Continued

\begin{tabular}{|c|c|c|c|c|c|c|c|}
\hline Date & Time & $\begin{array}{l}\text { Discharge, } \\
\text { instant- } \\
\text { aneous } \\
\left(\mathrm{ft}^{3} / \mathrm{s}\right)\end{array}$ & $\begin{array}{c}\text { Temper- } \\
\text { ature } \\
\left({ }^{\circ} \mathrm{C}\right)\end{array}$ & $\begin{array}{c}\text { Specific } \\
\text { conduc- } \\
\text { tance } \\
(\mu \mathrm{S} / \mathrm{cm})\end{array}$ & $\begin{array}{c}\mathrm{pH}, \text { water, } \\
\text { whole, field } \\
\text { (standard units) }\end{array}$ & $\begin{array}{l}\text { Selenium, } \\
\text { dissolved } \\
(\mu \mathrm{g} / \mathrm{L} \text { as } \mathrm{Se})\end{array}$ & $\begin{array}{l}\text { Selenium, } \\
\text { total } \\
(\mu \mathrm{g} / \mathrm{L} \text { as Se })\end{array}$ \\
\hline \multicolumn{8}{|c|}{ Green River near Greendale, Utah (09234500)—Continued } \\
\hline 06-26-97 & 1115 & 4,010 & 13.0 & 640 & 8.2 & $<1$ & - \\
\hline 08-06-97 & 1030 & 1,510 & 12.0 & 650 & 8.3 & $<1$ & - \\
\hline $10-09-97$ & 1145 & 2,890 & 12.0 & 640 & 8.4 & $<1$ & - \\
\hline $11-25-97$ & 1530 & 3,430 & 6.5 & 640 & 8.5 & $<1$ & - \\
\hline 01-07-98 & 1215 & 3,110 & 3.0 & 660 & 8.4 & $<1$ & - \\
\hline 02-24-98 & 1430 & 2,260 & 3.5 & 630 & 8.3 & $<1$ & - \\
\hline 04-14-98 & 1345 & 3,630 & 2.5 & 600 & 8.4 & $<1$ & - \\
\hline 05-19-98 & 1245 & 3,530 & 6.0 & 570 & 8.4 & $<1$ & - \\
\hline 07-07-98 & 1645 & 2,010 & 10.0 & 530 & 8.1 & $<1$ & - \\
\hline 08-18-98 & 1630 & 2,380 & 11.0 & 550 & 7.9 & $<1$ & - \\
\hline $10-13-98$ & 1710 & 2,310 & 11.5 & 610 & 6.6 & $<1$ & - \\
\hline $11-30-98$ & 1500 & 2,280 & 7.0 & 620 & 7.3 & $<1$ & - \\
\hline 01-05-99 & 1245 & 2,390 & 6.0 & 630 & 8.3 & $<1$ & - \\
\hline 02-16-99 & 1415 & 2,790 & 4.0 & 640 & 8.4 & $<1$ & - \\
\hline 03-23-99 & 1610 & 3,430 & 4.0 & 340 & 8.2 & $<1$ & - \\
\hline 05-12-99 & 1220 & 4,450 & 6.0 & 620 & 8.6 & $<1$ & - \\
\hline 06-10-99 & 1340 & 8,400 & 9.7 & 600 & 8.5 & $<1$ & - \\
\hline $07-22-99$ & 1220 & 2,070 & 12.5 & 610 & 8.5 & $<1$ & - \\
\hline 08-25-99 & 1610 & 2,080 & $14.5 \mathrm{E}$ & 620 & 8.2 & $<1$ & - \\
\hline $10-12-99$ & 1530 & 2,130 & 11.0 & 630 & 8.6 & $<2.4$ & - \\
\hline $12-02-99$ & 1410 & 2,180 & 8.0 & 630 & 8.7 & $<2.4$ & - \\
\hline $01-12-00$ & 1240 & 2,330 & 5.0 & 640 & 8.2 & $<2.4$ & - \\
\hline 03-01-00 & 1320 & 1,870 & 4.0 & 640 & 8.5 & $<2.4$ & - \\
\hline $04-13-00$ & 1240 & 1,690 & 5.5 & 640 & 8.6 & $<2.4$ & - \\
\hline $05-25-00$ & 1220 & 4,770 & 9.5 & 600 & 8.7 & $1.6 \mathrm{E}$ & - \\
\hline $07-13-00$ & 1310 & 1,360 & 14.0 & 600 & 8.5 & $1.6 \mathrm{E}$ & - \\
\hline $08-30-00$ & 1310 & 1,140 & 10.0 & 620 & 8.5 & $<2.4$ & - \\
\hline \multicolumn{8}{|c|}{ Yampa River near Maybell, Colorado (09251000) } \\
\hline $02-06-91$ & 1230 & $175 \mathrm{E}$ & 0 & 568 & 8.0 & $<1$ & - \\
\hline 04-10-91 & 1210 & 1,390 & 4.5 & 492 & 8.3 & 3.0 & - \\
\hline $05-15-91$ & 1355 & 5,270 & 10.5 & 205 & 8.0 & $<1$ & - \\
\hline 09-03-91 & 1325 & 105 & 25.0 & 512 & 8.4 & $<1$ & - \\
\hline $12-04-91$ & 1700 & 226 & 0 & 633 & 8.3 & $<1$ & - \\
\hline 03-17-92 & 1200 & 681 & 6.0 & 694 & 9.1 & 5.0 & - \\
\hline $06-12-92$ & 1305 & 2,060 & 19.0 & 200 & 8.3 & $<1$ & - \\
\hline 09-01-92 & 1400 & 146 & 20.0 & 439 & 8.7 & $<1$ & - \\
\hline $12-22-92$ & 1430 & 302 & 0 & 478 & 8.4 & $<1$ & - \\
\hline $03-22-93$ & 1100 & 800 & 0 & 730 & 8.5 & 3.0 & - \\
\hline $05-17-93$ & 1400 & 10,700 & 10.0 & 212 & 8.0 & $<1$ & - \\
\hline $08-25-93$ & 1000 & 291 & 18.5 & 404 & 8.4 & $<1$ & - \\
\hline $10-03-94$ & 1530 & 60 & 13.5 & 712 & - & $<1$ & - \\
\hline $03-20-95$ & 1500 & 1,220 & 9.0 & 615 & 8.7 & 6.9 & - \\
\hline
\end{tabular}


Table 5. Discharge, physical properties, and selenium concentration of water from the Green River and selected tributaries, Utah and Colorado, water years 1991-2000—Continued

\begin{tabular}{|c|c|c|c|c|c|c|c|}
\hline Date & Time & $\begin{array}{l}\text { Discharge, } \\
\text { instant- } \\
\text { aneous } \\
\left(\mathrm{ft}^{3} / \mathrm{s}\right)\end{array}$ & $\begin{array}{c}\text { Temper- } \\
\text { ature } \\
\left({ }^{\circ} \mathrm{C}\right)\end{array}$ & $\begin{array}{c}\text { Specific } \\
\text { conduc- } \\
\text { tance } \\
(\mu \mathrm{S} / \mathrm{cm})\end{array}$ & $\begin{array}{c}\mathrm{pH}, \text { water, } \\
\text { whole, field } \\
\text { (standard units) }\end{array}$ & $\begin{array}{c}\text { Selenium, } \\
\text { dissolved } \\
(\mu \mathrm{g} / \mathrm{L} \text { as Se })\end{array}$ & $\begin{array}{c}\text { Selenium, } \\
\text { total } \\
(\mu \mathrm{g} / \mathrm{L} \text { as } \mathrm{Se})\end{array}$ \\
\hline \multicolumn{8}{|c|}{ Yampa River near Maybell, Colorado (09251000)—Continued } \\
\hline $08-30-95$ & 1630 & 341 & 23.0 & 362 & 8.2 & 2.0 & - \\
\hline $10-03-95$ & 1630 & 497 & 14.0 & 414 & 8.2 & $<1$ & - \\
\hline 03-14-96 & 1615 & 1,550 & 1.5 & 667 & 7.7 & 3.0 & - \\
\hline $05-28-96$ & 1400 & 7,650 & 9.5 & 199 & 7.7 & $<1$ & - \\
\hline $06-25-96$ & 1630 & 4,860 & 16.0 & 133 & 7.7 & $<1$ & - \\
\hline 07-18-96 & 1217 & 817 & 22.0 & 292 & 8.3 & - & - \\
\hline 08-20-96 & 1110 & 211 & 19.5 & 541 & 8.5 & $<1$ & - \\
\hline 09-09-96 & 1130 & 117 & 18.0 & 610 & 8.0 & - & - \\
\hline $11-08-96$ & 1600 & 373 & 2.0 & 536 & 8.4 & $<1$ & - \\
\hline 01-17-97 & 1330 & 360 & 0 & 770 & 8.2 & 2.0 & - \\
\hline $03-24-97$ & 1400 & 2,680 & 6.0 & 705 & 8.0 & 7.4 & - \\
\hline 06-03-97 & 1400 & 14,400 & 15.0 & 173 & 8.0 & $<1$ & - \\
\hline 09-10-97 & 1100 & 380 & 19.0 & 484 & 8.7 & $<1$ & - \\
\hline 01-08-98 & 1620 & 426 & 0 & 712 & 8.3 & 2.3 & 2.8 \\
\hline $02-25-98$ & 1300 & 648 & 0 & 900 & 8.4 & 3.8 & 3.6 \\
\hline 03-12-98 & 1040 & 649 & .5 & 928 & 8.6 & 4.9 & 3.7 \\
\hline 03-18-98 & 1030 & 797 & 1.0 & 861 & 8.2 & 3.5 & $<3.6$ \\
\hline 03-26-98 & 1235 & $7,200 \mathrm{E}$ & 5.0 & 625 & 8.1 & 4.3 & 5.7 \\
\hline 04-01-98 & 1215 & 2,490 & 5.0 & 742 & 8.4 & 3.2 & 3.5 \\
\hline $04-21-98$ & 1910 & 3,030 & 10.5 & 659 & 8.3 & 2.7 & 2.7 \\
\hline $05-20-98$ & 1520 & 8,630 & 12.0 & 223 & 8.2 & $<1$ & $<1$ \\
\hline $07-22-98$ & 1015 & 860 & 22.0 & 354 & 8.4 & $<1$ & $<1$ \\
\hline 09-08-98 & 1445 & 150 & 23.5 & 605 & 8.5 & $<1$ & $<1$ \\
\hline $10-20-98$ & 1330 & 339 & 9.0 & 621 & 8.6 & $<1$ & - \\
\hline $11-12-98$ & 1030 & 423 & 1.0 & 587 & 8.5 & 1.4 & - \\
\hline 01-28-99 & 1230 & 363 & 0 & 827 & 8.3 & 1.9 & - \\
\hline 03-05-99 & 1325 & 492 & 1.0 & 962 & 8.5 & 4.3 & - \\
\hline 03-25-99 & 1810 & 1,290 & 9.0 & 762 & 8.5 & 7.9 & - \\
\hline 05-14-99 & 1250 & 3,370 & 8.0 & 361 & 8.3 & $<1$ & - \\
\hline 06-09-99 & 1000 & 5,550 & 13.0 & 139 & 8.1 & $<1$ & - \\
\hline 07-14-99 & 1040 & 865 & 21.0 & 294 & 8.4 & $<1$ & - \\
\hline 08-16-99 & 1615 & 294 & 22.0 & 440 & 8.7 & $<1$ & - \\
\hline $10-22-99$ & 1310 & 316 & 8.0 & 587 & 8.7 & $<2.4$ & - \\
\hline $02-21-00$ & 1302 & 342 & 0 & 957 & 8.6 & 5.6 & - \\
\hline $06-27-00$ & 1400 & 1,150 & 19.0 & 236 & 8.2 & $.7 \mathrm{E}$ & - \\
\hline 09-05-00 & 1244 & 147 & 20.0 & 588 & 8.5 & .7 & - \\
\hline \multicolumn{8}{|c|}{ Green River near Jensen, Utah (09261000) } \\
\hline $11-06-90$ & 1200 & 1,470 & 4.5 & 710 & 8.3 & $<1$ & - \\
\hline 03-19-91 & 1230 & 1,820 & 7.0 & 740 & 8.2 & 1.0 & - \\
\hline 04-23-91 & 1230 & 3,450 & 10.5 & 620 & 8.3 & $<1$ & - \\
\hline $05-30-91$ & 1130 & 9,910 & 13.5 & 240 & 8.1 & $<1$ & - \\
\hline $06-18-91$ & 1200 & 7,420 & 18.0 & 260 & 8.2 & $<1$ & - \\
\hline
\end{tabular}


Table 5. Discharge, physical properties, and selenium concentration of water from the Green River and selected tributaries, Utah and Colorado, water years 1991-2000-Continued

\begin{tabular}{|c|c|c|c|c|c|c|c|}
\hline Date & Time & $\begin{array}{l}\text { Discharge, } \\
\text { instant- } \\
\text { aneous } \\
\left(\mathrm{ft}^{3} / \mathrm{s}\right)\end{array}$ & $\begin{array}{c}\text { Temper- } \\
\text { ature } \\
\left({ }^{\circ} \mathrm{C}\right)\end{array}$ & $\begin{array}{c}\text { Specific } \\
\text { conduc- } \\
\text { tance } \\
(\mu \mathrm{S} / \mathrm{cm})\end{array}$ & $\begin{array}{c}\text { pH, water, } \\
\text { whole, field } \\
\text { (standard units) }\end{array}$ & $\begin{array}{l}\text { Selenium, } \\
\text { dissolved } \\
(\mu \mathrm{g} / \mathrm{L} \text { as Se })\end{array}$ & $\begin{array}{l}\text { Selenium, } \\
\text { total } \\
(\mu \mathrm{g} / \mathrm{L} \text { as Se })\end{array}$ \\
\hline \multicolumn{8}{|c|}{ Green River near Jensen, Utah (09261000)—Continued } \\
\hline $07-16-91$ & 1230 & 2,480 & 22.0 & 690 & 8.4 & $<1$ & - \\
\hline 09-11-91 & 1130 & 1,870 & 16.5 & 780 & 8.3 & $<1$ & - \\
\hline $10-01-91$ & 1130 & 1,930 & 14.0 & 760 & 8.4 & - & - \\
\hline $11-05-91$ & 1200 & 1,870 & 3.0 & 790 & 8.2 & $<1$ & - \\
\hline $12-03-91$ & 1200 & 1,770 & 0 & 730 & 8.1 & $<1$ & - \\
\hline 01-08-92 & 1230 & 2,350 & .5 & 810 & 8.3 & $<1$ & - \\
\hline $02-06-92$ & 1230 & 1,690 & 0 & 830 & 8.2 & $<1$ & - \\
\hline 03-03-92 & 1130 & 2,230 & 4.0 & 760 & 8.3 & $<1$ & - \\
\hline 04-07-92 & 1100 & 2,170 & 11.5 & 690 & 8.4 & 1.0 & - \\
\hline $05-06-92$ & 1145 & 6,790 & 16.0 & 370 & 8.4 & $<1$ & - \\
\hline 06-04-92 & 1145 & 5,120 & 18.0 & 400 & 8.3 & $<1$ & - \\
\hline 07-08-92 & 1130 & 1,640 & 21.5 & 780 & 8.5 & $<1$ & - \\
\hline 08-05-92 & 1145 & 1,440 & 20.5 & 670 & 8.4 & $<1$ & - \\
\hline 09-03-92 & 1230 & 1,720 & 16.0 & 720 & 8.4 & $<1$ & - \\
\hline $10-08-92$ & 1130 & 1,180 & 9.5 & 730 & 8.6 & $<1$ & - \\
\hline $11-04-92$ & 1030 & 1,610 & 7.0 & 720 & 8.4 & $<1$ & - \\
\hline $12-10-92$ & 1500 & 1,470 & 1.0 & 750 & 8.6 & $<1$ & - \\
\hline $03-25-93$ & 1300 & 3,130 & 8.0 & 685 & 8.4 & 2.0 & - \\
\hline $04-15-93$ & 1200 & 3,130 & 10.0 & 685 & 8.6 & 2.0 & - \\
\hline $05-11-93$ & 1900 & 6,090 & 17.0 & 445 & 8.4 & 1.0 & - \\
\hline $07-14-93$ & 1200 & 3,650 & 21.0 & 350 & 8.3 & $<1$ & - \\
\hline 08-19-93 & 1230 & 1,700 & 25.0 & 660 & - & $<1$ & - \\
\hline 09-16-93 & 1230 & 1,550 & 14.0 & 740 & 8.7 & 1.0 & - \\
\hline $10-07-93$ & 1215 & 1,500 & 14.0 & 730 & 8.6 & $<1$ & - \\
\hline 03-18-94 & 0930 & 2,560 & 9.0 & 750 & 8.6 & 2.0 & - \\
\hline 05-19-94 & 1440 & 11,700 & 14.5 & 395 & 8.3 & $<1$ & - \\
\hline 07-28-94 & 1200 & 1,590 & 22.5 & 740 & 8.5 & $<1$ & - \\
\hline 09-21-94 & 0945 & 1,740 & 15.0 & 770 & 8.5 & $<1$ & - \\
\hline $10-18-94$ & 0900 & 1,980 & 9.0 & - & 8.7 & - & - \\
\hline $02-28-95$ & 1645 & 2,070 & - & 670 & - & - & 1.0 \\
\hline 03-02-95 & 1615 & 1,840 & - & 700 & - & - & 1.0 \\
\hline $03-05-95$ & 1355 & 1,690 & - & - & - & - & $<1$ \\
\hline 03-12-95 & 1150 & 1,540 & - & - & - & - & 1.0 \\
\hline 03-16-95 & 1600 & 1,920 & - & 720 & - & - & 1.0 \\
\hline $03-20-95$ & 1600 & 2,280 & - & 670 & - & - & 2.0 \\
\hline 04-02-95 & 1131 & 1,780 & - & 720 & - & - & 2.0 \\
\hline 04-09-95 & 1655 & 2,860 & - & 660 & - & - & 1.0 \\
\hline 04-10-95 & 1815 & 3,360 & - & 610 & - & - & 1.0 \\
\hline 04-12-95 & 1730 & 3,130 & - & 520 & - & - & 1.0 \\
\hline $04-15-95$ & 1800 & 2,650 & - & 580 & - & - & 1.0 \\
\hline 04-28-95 & 1800 & 4,180 & - & 630 & - & - & 1.0 \\
\hline 05-03-95 & 2025 & 6,500 & - & 660 & - & - & 4.0 \\
\hline
\end{tabular}


Table 5. Discharge, physical properties, and selenium concentration of water from the Green River and selected tributaries, Utah and Colorado, water years 1991-2000—Continued

\begin{tabular}{|c|c|c|c|c|c|c|c|}
\hline Date & Time & $\begin{array}{l}\text { Discharge, } \\
\text { instant- } \\
\text { aneous } \\
\left(\mathrm{ft}^{3} / \mathrm{s}\right)\end{array}$ & $\begin{array}{c}\text { Temper- } \\
\text { ature } \\
\left({ }^{\circ} \mathrm{C}\right)\end{array}$ & $\begin{array}{c}\text { Specific } \\
\text { conduc- } \\
\text { tance } \\
(\mu \mathrm{S} / \mathrm{cm})\end{array}$ & $\begin{array}{c}\mathrm{pH}, \text { water, } \\
\text { whole, field } \\
\text { (standard units) }\end{array}$ & $\begin{array}{l}\text { Selenium, } \\
\text { dissolved } \\
(\mu \mathrm{g} / \mathrm{L} \text { as } \mathrm{Se})\end{array}$ & $\begin{array}{c}\text { Selenium, } \\
\text { total } \\
(\mu \mathrm{g} / \mathrm{L} \text { as } \mathrm{Se})\end{array}$ \\
\hline \multicolumn{8}{|c|}{ Green River near Jensen, Utah (09261000)—Continued } \\
\hline $05-16-95$ & 1530 & 8,580 & 14.5 & 465 & 8.3 & 1.0 & - \\
\hline 07-13-95 & 1530 & 10,100 & 19.5 & 290 & 8.2 & $<1$ & - \\
\hline 08-29-95 & 1630 & 1,730 & 22.0 & 640 & 8.5 & $<1$ & - \\
\hline $10-04-95$ & 1530 & 2,020 & 11.0 & 650 & 8.5 & $<1$ & - \\
\hline 04-03-96 & 1600 & 5,680 & 10.0 & 740 & 8.4 & 4.0 & - \\
\hline $05-16-96$ & 1510 & 18,800 & 15.0 & 320 & 8.1 & $<1$ & - \\
\hline $07-10-96$ & 1600 & 3,600 & 22.0 & 400 & 8.4 & $<1$ & - \\
\hline $08-21-96$ & 1700 & 1,780 & 22.0 & 670 & 8.5 & $<1$ & - \\
\hline $10-15-96$ & 1650 & 1,920 & 13.0 & 680 & 8.5 & $<1$ & - \\
\hline $12-05-96$ & 1030 & 2,040 & 2.0 & 770 & 8.3 & $<1$ & - \\
\hline 01-29-97 & 1745 & 2,560 & 3.0 & 730 & 8.3 & $<1$ & - \\
\hline 03-06-97 & 1545 & 3,570 & 4.5 & 710 & 8.5 & $<1$ & - \\
\hline $04-16-97$ & 1500 & 5,440 & 12.0 & 700 & 8.4 & 1.2 & - \\
\hline 08-06-97 & 0700 & 2,910 & 19.0 & 550 & 8.4 & $<1$ & - \\
\hline $10-10-97$ & 0815 & 4,450 & 10.5 & 590 & 8.5 & $<1$ & - \\
\hline $11-20-97$ & 1440 & 4,360 & 6.0 & 650 & 8.5 & $<1$ & - \\
\hline 04-16-98 & 1755 & 8,320 & 8.0 & 560 & 8.4 & 1.2 & 1.2 \\
\hline $05-21-98$ & 1400 & 14,700 & 5.0 & 300 & 8.1 & $<1$ & - \\
\hline 07-08-98 & 1700 & 5,500 & 23.0 & 320 & 8.3 & $<1$ & $<1$ \\
\hline $10-16-98$ & 0830 & 3,070 & 10.0 & 620 & 8.1 & $<1$ & $<1$ \\
\hline 03-26-99 & 0810 & 5,520 & 7.5 & 690 & 8.3 & 3.5 & - \\
\hline 03-31-99 & 1020 & 5,520 & 7.4 & 622 & - & - & - \\
\hline 05-13-99 & 1340 & 11,100 & 10.0 & 410 & 8.3 & $<1$ & - \\
\hline $05-20-99$ & 0915 & 10,800 & 13.6 & 479 & - & - & - \\
\hline 05-28-99 & 0845 & 19,700 & 14.7 & 345 & - & - & - \\
\hline 06-11-99 & 0830 & 17,900 & 13.0 & 380 & 8.3 & $<1$ & - \\
\hline 06-24-99 & 1030 & 13,800 & 16.8 & 405 & - & - & - \\
\hline 07-22-99 & 1750 & 3,180 & 23.0 & 540 & 8.5 & $<1$ & - \\
\hline 08-26-99 & 1730 & 2,490 & 21.5 & 620 & 8.7 & $<1$ & - \\
\hline $03-02-00$ & 1310 & 2,400 & 6.5 & 670 & 8.6 & $1.4 \mathrm{E}$ & - \\
\hline 03-08-00 & 1120 & - & - & 685 & - & 1.4 & - \\
\hline $04-12-00$ & 1300 & 5,100 & 12.5 & 550 & 8.5 & $1.6 \mathrm{E}$ & - \\
\hline $07-12-00$ & 1510 & 1,750 & 22.0 & 580 & 8.6 & $1.2 \mathrm{E}$ & - \\
\hline $08-31-00$ & 1630 & 1,340 & 8.5 & 650 & 8.5 & $<2.4$ & - \\
\hline $09-13-00$ & 1100 & - & 17.0 & 640 & 8.4 & $<2.4$ & - \\
\hline \multicolumn{8}{|c|}{ Ashley Creek near Jensen, Utah $(09271500)$} \\
\hline $08-14-91$ & 0930 & 6.8 & 20.5 & 2,330 & 7.7 & 57 & - \\
\hline $10-23-91$ & 0915 & - & 10.5 & 2,100 & - & 44 & 55 \\
\hline \multicolumn{8}{|c|}{ Ashley Creek Below Union Canal Diversion near Jensen, Utah (09271550) } \\
\hline $07-16-91$ & 1530 & .70 & 29.5 & 3,930 & 7.9 & 19 & - \\
\hline $11-05-91$ & 1400 & - & 5.0 & 2,310 & 8.2 & 39 & - \\
\hline $02-05-92$ & 1515 & 14 & -.5 & 2,490 & 7.8 & 74 & - \\
\hline
\end{tabular}


Table 5. Discharge, physical properties, and selenium concentration of water from the Green River and selected tributaries, Utah and Colorado, water years 1991-2000-Continued

\begin{tabular}{|c|c|c|c|c|c|c|c|}
\hline Date & Time & $\begin{array}{l}\text { Discharge, } \\
\text { instant- } \\
\text { aneous } \\
\left(\mathrm{ft}^{3} / \mathrm{s}\right)\end{array}$ & $\begin{array}{c}\text { Temper- } \\
\text { ature } \\
\left({ }^{\circ} \mathrm{C}\right)\end{array}$ & $\begin{array}{c}\text { Specific } \\
\text { conduc- } \\
\text { tance } \\
(\mu \mathrm{S} / \mathrm{cm})\end{array}$ & $\begin{array}{c}\text { pH, water, } \\
\text { whole, field } \\
\text { (standard units) }\end{array}$ & $\begin{array}{l}\text { Selenium, } \\
\text { dissolved } \\
(\mu \mathrm{g} / \mathrm{L} \text { as } \mathrm{Se})\end{array}$ & $\begin{array}{l}\text { Selenium, } \\
\text { total } \\
(\mu \mathrm{g} / \mathrm{L} \text { as Se })\end{array}$ \\
\hline \multicolumn{8}{|c|}{ Ashley Creek Below Union Canal Diversion near Jensen, Utah (09271550)_Continued } \\
\hline $05-06-92$ & 1645 & 3.3 & 23.0 & 2,860 & 8.1 & 160 & - \\
\hline 06-03-92 & 1710 & 4.7 & 27.0 & 2,630 & 8.2 & 41 & - \\
\hline 08-07-92 & 1000 & 1.0 & 21.0 & 3,550 & 7.8 & 29 & - \\
\hline $12-10-92$ & 1700 & - & - & - & - & 61 & - \\
\hline $03-25-93$ & 1130 & 46 & 11.0 & 2,260 & 8.3 & $<65$ & - \\
\hline $04-21-93$ & 1030 & 26 & 8.0 & 2,420 & 8.2 & 80 & - \\
\hline $05-26-93$ & 0850 & 1,200 & 6.5 & 96 & 8.3 & 4.0 & - \\
\hline $06-23-93$ & 1905 & 179 & 18.0 & 560 & 8.5 & 9.0 & - \\
\hline $07-28-93$ & 0730 & 71 & 17.0 & 1,390 & 8.2 & 23 & - \\
\hline $08-25-93$ & 0700 & 20 & 17.0 & 1,800 & 8.1 & 27 & - \\
\hline $10-13-93$ & 0830 & 40 & 10.5 & 2,100 & 8.1 & 22 & - \\
\hline $03-17-94$ & 0915 & 23 & 7.0 & 2,350 & 8.2 & 56 & - \\
\hline 04-13-94 & 0905 & 5.0 & 9.0 & 3,080 & 8.1 & 100 & - \\
\hline $05-18-94$ & 0900 & 21 & 13.0 & 1,130 & 8.2 & 25 & - \\
\hline $06-16-94$ & 0855 & .90 & 15.0 & 3,020 & 7.8 & 54 & - \\
\hline 07-14-94 & 0710 & .75 & 18.0 & 3,080 & 7.8 & 27 & - \\
\hline $08-16-94$ & 1450 & .40 & 27.0 & 3,980 & 8.1 & 82 & - \\
\hline $09-20-94$ & 1455 & - & 19.0 & 4,400 & 8.3 & 55 & - \\
\hline $10-19-94$ & 1120 & 58 & 9.5 & 1,630 & 8.3 & 48 & - \\
\hline $03-21-95$ & 0820 & 7.7 & 9.0 & 2,630 & 8.2 & 99 & - \\
\hline 04-19-95 & 0845 & 1.0 & 9.0 & 3,440 & 8.1 & 110 & - \\
\hline $05-18-95$ & 0745 & 10 & 12.0 & 2,560 & 8.1 & 88 & - \\
\hline $06-22-95$ & 0830 & $1,200 \mathrm{E}$ & 9.0 & 330 & 8.1 & 4.0 & - \\
\hline $07-18-95$ & 0730 & 146 & 17.5 & 850 & 8.0 & 16 & - \\
\hline 08-17-95 & 0740 & 53 & 18.5 & 1,680 & 8.1 & 20 & - \\
\hline 09-23-95 & 0605 & 153 & - & 1,640 & 8.2 & 33 & - \\
\hline $10-25-95$ & 0710 & 37 & 4.0 & 1,900 & 8.2 & 2.0 & - \\
\hline $03-26-96$ & 0835 & 12 & - & 2,430 & 8.2 & 68 & - \\
\hline $05-16-96$ & 0745 & 3.4 & 18.0 & 2,650 & 8.0 & 69 & - \\
\hline 06-13-96 & 0750 & 19 & 16.0 & 1,710 & 8.0 & 27 & - \\
\hline 07-16-96 & 0940 & 3.8 & 21.0 & 2,020 & 8.1 & 25 & - \\
\hline $08-20-96$ & 0655 & 1.0 & 18.5 & 2,240 & 8.0 & 43 & - \\
\hline 09-19-96 & 0945 & 17 & 12.0 & 2,090 & 8.4 & 35 & - \\
\hline 03-20-97 & 0805 & 52 & 6.0 & 2,120 & 8.2 & 39 & - \\
\hline 04-16-97 & 1200 & 20 & 12.0 & 2,220 & 8.5 & 51 & - \\
\hline 04-17-97 & 1100 & - & - & - & - & - & 54 \\
\hline 05-14-97 & 0810 & - & 9.5 & 402 & 8.1 & 5.4 & - \\
\hline 06-10-97 & 0720 & 500 & 12.0 & 330 & 8.3 & 4.0 & - \\
\hline $07-17-97$ & 0830 & 4.6 & 19.0 & 2,900 & 7.4 & 23 & - \\
\hline $09-16-97$ & 0740 & 21 & 14.5 & 2,070 & 8.2 & 48 & - \\
\hline $10-07-97$ & 1050 & 12 & 14.0 & 1,780 & 8.5 & 36 & - \\
\hline $03-25-98$ & 0735 & 52 & 8.0 & 1,110 & 8.3 & 26 & - \\
\hline
\end{tabular}


Table 5. Discharge, physical properties, and selenium concentration of water from the Green River and selected tributaries, Utah and Colorado, water years 1991-2000—Continued

\begin{tabular}{|c|c|c|c|c|c|c|c|}
\hline Date & Time & $\begin{array}{l}\text { Discharge, } \\
\text { instant- } \\
\text { aneous } \\
\left(\mathrm{ft}^{3} / \mathrm{s}\right)\end{array}$ & $\begin{array}{c}\text { Temper- } \\
\text { ature } \\
\left({ }^{\circ} \mathrm{C}\right)\end{array}$ & $\begin{array}{l}\text { Specific } \\
\text { conduc- } \\
\text { tance } \\
(\mu \mathrm{S} / \mathrm{cm})\end{array}$ & $\begin{array}{c}\text { pH, water, } \\
\text { whole, field } \\
\text { (standard units) }\end{array}$ & $\begin{array}{l}\text { Selenium, } \\
\text { dissolved } \\
(\mu \mathrm{g} / \mathrm{L} \text { as } \mathrm{Se})\end{array}$ & $\begin{array}{c}\text { Selenium, } \\
\text { total } \\
(\mu \mathrm{g} / \mathrm{L} \text { as } \mathrm{Se})\end{array}$ \\
\hline \multicolumn{8}{|c|}{ Ashley Creek Below Union Canal Diversion near Jensen, Utah (09271550)_Continued } \\
\hline $05-20-98$ & 0735 & 1,050 & 8.3 & 223 & 8.2 & 2.6 & - \\
\hline 06-16-98 & 0830 & 435 & 11.0 & 300 & 8.1 & 3.4 & - \\
\hline 07-22-98 & 1830 & 1.5 & 24.5 & 1,360 & 8.5 & - & - \\
\hline 09-17-98 & 1710 & 18 & 24.0 & 1,720 & 8.4 & 29 & - \\
\hline $10-20-98$ & 1100 & 6.3 & 8.5 & 1,620 & 8.0 & 28 & - \\
\hline 03-16-99 & 1600 & 25 & 12.0 & 2,080 & 8.6 & 54 & - \\
\hline 05-19-99 & 0745 & 82 & 13.5 & 1,200 & 8.3 & 22 & - \\
\hline 06-16-99 & 1600 & 575 & 16.5 & 220 & 8.2 & 3.1 & - \\
\hline 07-13-99 & 0745 & 4.9 & 20.0 & 2,000 & 8.0 & 21 & - \\
\hline 09-16-99 & 1310 & 19 & 19.0 & 1,950 & 8.3 & 31 & - \\
\hline $10-14-99$ & 1645 & 13 & 16.5 & 2,020 & 8.4 & 43 & - \\
\hline $03-14-00$ & 0745 & 13 & 4.0 & 2,320 & 8.2 & 60 & - \\
\hline $05-23-00$ & 1650 & 127 & 20.0 & 440 & 8.3 & 7.2 & - \\
\hline $06-28-00$ & 1100 & - & 21.0 & 2,150 & 8.3 & 42 & - \\
\hline $07-26-00$ & 1300 & 6.3 & 25.0 & 2,450 & 8.2 & 51 & - \\
\hline $08-30-00$ & 1615 & - & 26.0 & 2,790 & - & 62 & - \\
\hline \multicolumn{8}{|c|}{ Irrigation drains J1 and J1A combined at the Green River above Stewart Lake WMA (402136109204102) } \\
\hline $11-20-97$ & 900 & - & - & 3,180 & - & - & 28 \\
\hline 07-06-98 & 1650 & - & 12.7 & 2,380 & - & - & 31 \\
\hline 07-23-98 & 945 & 1.7 & 12 & 2,170 & 7.1 & 26 & - \\
\hline $08-21-98$ & 1035 & - & 13.5 & 2,120 & 7.2 & 24 & - \\
\hline 07-14-98 & 930 & - & - & - & - & - & 27 \\
\hline 09-17-98 & 1630 & .07 & 14 & 2,130 & 7.2 & 20 & - \\
\hline $10-20-98$ & 1315 & 2 & 15 & 2,020 & 7.2 & 17 & - \\
\hline 03-17-99 & 720 & .37 & 7.5 & 1,500 & 7.8 & 13 & - \\
\hline 04-20-99 & 1610 & .89 & 8.5 & 2,310 & 7.3 & 23 & - \\
\hline 05-19-99 & 815 & - & - & 2,280 & 7.3 & 25 & - \\
\hline 06-16-99 & 1800 & 1.6 & 13 & 2,110 & 7.2 & 19 & - \\
\hline 07-13-99 & 815 & - & 12 & 1,960 & 7.2 & 17 & - \\
\hline 08-18-99 & 805 & 3.7 & 14 & 2,070 & 7.1 & 19 & - \\
\hline 09-16-99 & 1230 & 2.6 & 14.5 & 1,880 & 7.2 & 16 & - \\
\hline $10-14-99$ & 1705 & - & 13.5 & 2,010 & 7.3 & 18 & - \\
\hline $03-14-00$ & 900 & .13 & 4 & 2,320 & 8.2 & 12 & - \\
\hline 04-26-00 & 810 & - & 9 & 2,390 & 7.2 & 30 & - \\
\hline $05-24-00$ & 730 & 4.8 & 10.5 & 1,980 & 7.2 & 30 & - \\
\hline $06-28-00$ & 825 & - & 11.5 & 2,110 & 7.3 & 26 & - \\
\hline $07-26-00$ & 1205 & 5.2 & 13.5 & 1,950 & 7.3 & 19 & - \\
\hline $08-30-00$ & 1455 & - & 14 & 1,940 & 7.3 & 21 & - \\
\hline $10-11-00$ & 800 & 2.1 & 14 & 2,030 & 7.4 & 26 & - \\
\hline \multicolumn{8}{|c|}{ Stewart Lake Outflow near Jensen, Utah (09271600) } \\
\hline $06-20-91$ & 0815 & 4.2 & 16.0 & 2,830 & 7.6 & 8.0 & - \\
\hline $08-28-91$ & 1700 & 3.5 & 29.0 & 2,350 & 8.0 & 12 & - \\
\hline
\end{tabular}


Table 5. Discharge, physical properties, and selenium concentration of water from the Green River and selected tributaries, Utah and Colorado, water years 1991-2000-Continued

\begin{tabular}{|c|c|c|c|c|c|c|c|}
\hline Date & Time & $\begin{array}{l}\text { Discharge, } \\
\text { instant- } \\
\text { aneous } \\
\left(\mathrm{ft}^{3} / \mathrm{s}\right)\end{array}$ & $\begin{array}{c}\text { Temper- } \\
\text { ature } \\
\left({ }^{\circ} \mathrm{C}\right)\end{array}$ & $\begin{array}{c}\text { Specific } \\
\text { conduc- } \\
\text { tance } \\
(\mu \mathrm{S} / \mathrm{cm})\end{array}$ & $\begin{array}{c}\text { pH, water, } \\
\text { whole, field } \\
\text { (standard units) }\end{array}$ & $\begin{array}{l}\text { Selenium, } \\
\text { dissolved } \\
(\mu \mathrm{g} / \mathrm{L} \text { as Se })\end{array}$ & $\begin{array}{l}\text { Selenium, } \\
\text { total } \\
(\mu \mathrm{g} / \mathrm{L} \text { as Se })\end{array}$ \\
\hline \multicolumn{8}{|c|}{ Stewart Lake Outflow near Jensen, Utah (09271600)—Continued } \\
\hline $10-23-91$ & 1300 & - & 11.0 & 2,550 & - & 10 & 6.0 \\
\hline 04-07-92 & 1500 & .70 & 20.5 & 2,350 & 8.4 & 9.0 & - \\
\hline 06-04-92 & 1630 & 3.8 & 21.0 & 2,780 & 7.7 & 2.0 & - \\
\hline 08-17-92 & 1430 & 3.6 & 28.0 & 2,450 & 7.9 & 6.0 & - \\
\hline $03-25-93$ & 1150 & 10 & 7.0 & 1,670 & 8.2 & 11 & - \\
\hline $04-21-93$ & 1130 & 1.2 & 11.5 & 2,760 & 7.8 & 8.0 & - \\
\hline $06-24-93$ & 1200 & 2.1 & 17.5 & 1,630 & 7.4 & 1.0 & - \\
\hline $07-28-93$ & 1205 & 3.1 & 20.0 & 2,600 & 7.5 & 2.0 & - \\
\hline $08-25-93$ & 0930 & .09 & 19.0 & 2,700 & 7.5 & 1.0 & - \\
\hline $10-13-93$ & 0920 & 9.2 & 15.5 & 2,280 & 7.5 & 3.0 & - \\
\hline 03-17-94 & 1125 & .24 & 7.0 & 1,510 & - & 2.0 & - \\
\hline 04-13-94 & 1115 & .10 & 12.0 & 1,570 & 7.7 & 2.0 & - \\
\hline 05-18-94 & 1050 & .96 & 16.0 & 2,110 & 7.0 & $<2$ & - \\
\hline $06-16-94$ & 1045 & .96 & 19.0 & 2,840 & 7.4 & 1.0 & - \\
\hline 07-14-94 & 0840 & 1.9 & 21.0 & 2,780 & 7.4 & 1.0 & - \\
\hline $08-17-94$ & 0810 & 2.1 & 21.0 & 2,550 & 7.5 & 1.0 & - \\
\hline $09-21-94$ & 0930 & .22 & 14.0 & 2,380 & 7.6 & $<1$ & - \\
\hline $10-19-94$ & 0835 & .20 & 9.5 & 1,970 & 7.6 & $<1$ & - \\
\hline $02-15-95$ & 1440 & .06 & 3.0 & 2,700 & 7.3 & 3.0 & - \\
\hline $03-21-95$ & 0855 & .24 & 9.0 & 1,390 & 7.6 & 1.0 & - \\
\hline 04-19-95 & 0950 & - & 10.5 & 2,090 & 7.6 & 1.0 & 1.0 \\
\hline $05-04-95$ & 1245 & - & - & 2,100 & - & 1.0 & - \\
\hline $05-18-95$ & 0920 & .01 & 15.0 & 2,240 & 7.6 & 1.0 & 1.0 \\
\hline $06-22-95$ & 1000 & - & 16.5 & 570 & 7.5 & 2.0 & - \\
\hline 07-18-95 & 0915 & 7.6 & 21.0 & 1,090 & 7.3 & $<2$ & - \\
\hline 08-17-95 & 0900 & 4.6 & 21.5 & 1,950 & 7.5 & - & - \\
\hline 09-23-95 & 0640 & 7.5 & 12.0 & 1,780 & 7.7 & 3.0 & - \\
\hline $10-25-95$ & 0815 & 4.0 & 4.5 & 2,000 & 7.2 & 3.0 & - \\
\hline $03-26-96$ & 1045 & .09 & 5.0 & 1,810 & 8.1 & 1.0 & - \\
\hline 04-16-96 & 0910 & .32 & 10.0 & 2,290 & 7.9 & 1.0 & - \\
\hline 06-13-96 & 0930 & - & 20.0 & 1,770 & 7.4 & 2.0 & - \\
\hline $07-16-96$ & 1200 & - & 25.0 & 2,110 & 7.9 & - & - \\
\hline $08-20-96$ & 1250 & - & 26.5 & 1,730 & 8.3 & 11 & - \\
\hline 09-19-96 & 1300 & - & 17.0 & 1,500 & 8.3 & 10 & - \\
\hline $10-24-96$ & 1000 & 5.4 & 4.0 & 1960 & 8.0 & 12 & - \\
\hline 03-20-97 & 0945 & 3.0 & 6.5 & 770 & 7.8 & 2.0 & - \\
\hline 04-16-97 & 0905 & .53 & 6.0 & 2,110 & 7.8 & 2.4 & - \\
\hline $05-27-97$ & 0815 & - & - & - & - & - & $<1$ \\
\hline 06-10-97 & 1630 & $100 \mathrm{E}$ & - & 307 & 7.8 & $<1$ & $<1$ \\
\hline $07-17-97$ & 1120 & $30 \mathrm{E}$ & 25.0 & 2,100 & 8.0 & 9.9 & 10 \\
\hline 08-19-97 & 1025 & 8.0 & 21.0 & 1,960 & 8.2 & 11 & - \\
\hline 09-16-97 & 0905 & 21 & 14.5 & 1,810 & 8.0 & 15 & - \\
\hline
\end{tabular}


Table 5. Discharge, physical properties, and selenium concentration of water from the Green River and selected tributaries, Utah and Colorado, water years 1991-2000—Continued

\begin{tabular}{|c|c|c|c|c|c|c|c|}
\hline Date & Time & $\begin{array}{l}\text { Discharge, } \\
\text { instant- } \\
\text { aneous } \\
\left(\mathrm{ft}^{3} / \mathrm{s}\right)\end{array}$ & $\begin{array}{l}\text { Temper- } \\
\text { ature } \\
\left({ }^{\circ} \mathrm{C}\right)\end{array}$ & $\begin{array}{l}\text { Specific } \\
\text { conduc- } \\
\text { tance } \\
(\mu \mathrm{S} / \mathrm{cm})\end{array}$ & $\begin{array}{c}\mathrm{pH}, \text { water, } \\
\text { whole, field } \\
\text { (standard units) }\end{array}$ & $\begin{array}{l}\text { Selenium, } \\
\text { dissolved } \\
(\mu \mathrm{g} / \mathrm{L} \text { as } \mathrm{Se})\end{array}$ & $\begin{array}{l}\text { Selenium, } \\
\text { total } \\
(\mu \mathrm{g} / \mathrm{L} \text { as } \mathrm{Se})\end{array}$ \\
\hline \multicolumn{8}{|c|}{ Stewart Lake Outflow near Jensen, Utah (09271600)—Continued } \\
\hline $10-07-97$ & 1215 & 9.8 & 15.0 & 2,290 & 8.4 & 14 & 17 \\
\hline $10-09-97$ & 0740 & - & - & - & - & - & 10 \\
\hline $10-10-97$ & 0750 & - & - & - & - & - & 7.5 \\
\hline $10-11-97$ & 0730 & - & - & - & - & - & 10 \\
\hline $10-13-97$ & 1015 & - & - & - & - & - & 10 \\
\hline $10-14-97$ & 0750 & - & - & - & - & - & 8.3 \\
\hline $10-15-97$ & 0810 & - & - & - & - & - & 10 \\
\hline $10-16-97$ & 0820 & - & - & - & - & - & 12 \\
\hline $10-18-97$ & 0745 & - & - & - & - & - & 11 \\
\hline $10-21-97$ & 0805 & - & - & - & - & - & 17 \\
\hline $10-22-97$ & 0750 & - & - & - & - & - & 22 \\
\hline $10-23-97$ & 0720 & - & - & - & - & - & 19 \\
\hline $10-24-97$ & 0745 & - & - & - & - & - & 19 \\
\hline $03-25-98$ & 0900 & .02 & 8.0 & 2,800 & 7.7 & 15 & - \\
\hline 04-03-98 & 0930 & - & - & 3,180 & - & 9.7 & 11 \\
\hline 04-29-98 & 0900 & 67 & 12.5 & 650 & 8.3 & 3.5 & - \\
\hline $05-08-98$ & 1240 & - & - & - & - & - & 1.8 \\
\hline $05-11-98$ & 1400 & 115 & 14.0 & 480 & 7.9 & 2.2 & - \\
\hline $05-13-98$ & 1210 & - & - & - & - & - & 2.2 \\
\hline $05-20-98$ & 1010 & 27 & 17.0 & 560 & 8.0 & 2.5 & 2.7 \\
\hline 06-16-98 & 1100 & 27 & 17.0 & 600 & 8.2 & 3.1 & - \\
\hline 07-06-98 & 1607 & - & 29.4 & 2,480 & - & - & 10 \\
\hline $07-22-98$ & 1950 & 5.5 & 26.5 & 185 & 7.9 & 5.7 & - \\
\hline $08-21-98$ & 0922 & - & 17.0 & 1,330 & 7.8 & 8.6 & - \\
\hline $08-27-98$ & 1410 & - & - & - & - & - & 9.3 \\
\hline 09-17-98 & 1525 & 5.2 & 28.5 & 1,210 & 8.3 & 8.9 & - \\
\hline $10-20-98$ & 1330 & 5.0 & 15.0 & 1,880 & 8.1 & 16 & - \\
\hline $11-17-98$ & 2250 & - & - & - & - & - & 33 \\
\hline 03-17-99 & 0825 & 1.0 & 1.5 & 3,000 & 7.9 & 29 & - \\
\hline 04-06-99 & 1705 & - & - & 2,520 & - & - & 14 \\
\hline 05-24-99 & 1410 & - & - & - & - & - & 7.1 \\
\hline $05-25-99$ & 0930 & - & - & - & - & - & 7.3 \\
\hline 05-26-99 & 1405 & - & - & - & - & - & 3.9 \\
\hline $05-27-99$ & 0900 & - & - & - & - & - & 3.1 \\
\hline $05-27-99$ & 1030 & - & - & - & - & - & 2.6 \\
\hline 05-27-99 & 1700 & - & - & - & - & - & 3.8 \\
\hline $05-28-99$ & 0920 & - & - & - & - & - & 4.2 \\
\hline 06-01-99 & 1715 & - & - & - & - & - & 3.9 \\
\hline 06-02-99 & 1000 & - & - & - & - & - & 3.4 \\
\hline 06-03-99 & 0930 & - & - & - & - & - & 3.9 \\
\hline 06-05-99 & 0830 & - & - & - & - & - & 2.8 \\
\hline 06-16-99 & 1705 & 40 & 23.5 & 730 & 8.0 & 1.7 & - \\
\hline
\end{tabular}


Table 5. Discharge, physical properties, and selenium concentration of water from the Green River and selected tributaries, Utah and Colorado, water years 1991-2000-Continued

\begin{tabular}{|c|c|c|c|c|c|c|c|}
\hline Date & Time & $\begin{array}{l}\text { Discharge, } \\
\text { instant- } \\
\text { aneous } \\
\left(\mathrm{ft}^{3} / \mathrm{s}\right)\end{array}$ & $\begin{array}{c}\text { Temper- } \\
\text { ature } \\
\left({ }^{\circ} \mathrm{C}\right)\end{array}$ & $\begin{array}{c}\text { Specific } \\
\text { conduc- } \\
\text { tance } \\
(\mu \mathrm{S} / \mathrm{cm})\end{array}$ & $\begin{array}{c}\mathrm{pH}, \text { water, } \\
\text { whole, field } \\
\text { (standard units) }\end{array}$ & $\begin{array}{l}\text { Selenium, } \\
\text { dissolved } \\
(\mu \mathrm{g} / \mathrm{L} \text { as } \mathrm{Se})\end{array}$ & $\begin{array}{l}\text { Selenium, } \\
\text { total } \\
(\mu \mathrm{g} / \mathrm{L} \text { as Se })\end{array}$ \\
\hline \multicolumn{8}{|c|}{ Stewart Lake Outflow near Jensen, Utah (09271600)—Continued } \\
\hline 07-13-99 & 1400 & .00 & 28.0 & 1,160 & 7.5 & $<1$ & - \\
\hline 08-18-99 & 0900 & 1.9 & 15.0 & 1,340 & 7.9 & 3.8 & - \\
\hline 09-16-99 & 0810 & .70 & 9.0 & 1,890 & 7.6 & 6.9 & - \\
\hline $10-15-99$ & 0950 & 1.4 & 16.5 & 1,790 & 7.9 & 6.2 & - \\
\hline $03-14-00$ & 0925 & .37 & 7.0 & 2,660 & 7.8 & 12 & - \\
\hline $05-10-00$ & 0910 & - & - & - & - & - & $<2.6$ \\
\hline $05-19-00$ & 1600 & - & - & - & - & - & 4.6 \\
\hline $05-24-00$ & 1330 & 32 & 21.0 & 530 & 8.2 & 4.1 & - \\
\hline $05-24-00$ & 1730 & - & - & - & - & - & 5.4 \\
\hline 06-13-00 & - & - & - & - & - & 3.7 & - \\
\hline $06-27-00$ & - & - & - & - & - & 3.7 & - \\
\hline $07-26-00$ & 1010 & 1.3 & 24.5 & 2,210 & 8.1 & 7.5 & - \\
\hline 08-30-00 & 1420 & - & 27.5 & 1,470 & 8.3 & 8.6 & - \\
\hline \multicolumn{8}{|c|}{ Irrigation drains J2, J3, and J4 combined at the Green River below Stewart Lake WMA (402037109215003) } \\
\hline $07-06-98$ & 1620 & - & - & - & - & - & 49 \\
\hline 07-14-98 & 1030 & - & - & - & - & - & 38 \\
\hline 07-23-98 & 1240 & 2.4 & 12.5 & 3,100 & 7 & - & - \\
\hline $08-21-98$ & 910 & - & 13.5 & 3,110 & 7.1 & 36 & - \\
\hline 09-17-98 & 1500 & .07 & 14 & 2,800 & 7.2 & 32 & - \\
\hline $10-20-98$ & 1350 & 3 & 14 & 2,840 & 7.1 & 29 & - \\
\hline 03-17-99 & 745 & .24 & 7.5 & 3,230 & 7.4 & 37 & - \\
\hline 04-20-99 & 1645 & .32 & 9 & 3,250 & 7.4 & 36 & - \\
\hline $05-20-99$ & 845 & - & 10 & 3,670 & 7.2 & 35 & - \\
\hline 06-16-99 & 1725 & 1.7 & 11 & 3,120 & 7.1 & 30 & - \\
\hline 07-13-99 & 855 & 4 & 12.5 & 3,060 & 7.1 & 32 & - \\
\hline 08-18-99 & 915 & 1.5 & 13.5 & 2,860 & 7.1 & 34 & - \\
\hline 09-16-99 & 820 & 2 & 14 & 2,800 & 7.1 & 34 & - \\
\hline $10-15-99$ & 1005 & - & 14 & 2,940 & 7.2 & 30 & - \\
\hline $03-14-00$ & 1000 & .17 & 7.5 & 3,080 & 7.6 & 38 & - \\
\hline $04-26-00$ & 1010 & - & 9.5 & 2,910 & 7.2 & 44 & - \\
\hline $05-24-00$ & 900 & 4 & 10 & 3,440 & 7.1 & 42 & - \\
\hline $06-28-00$ & 940 & - & 12 & 2,460 & 7.2 & 30 & - \\
\hline $07-26-00$ & 1110 & 4.5 & 14 & 2,690 & 7.1 & 34 & - \\
\hline 08-30-00 & 1330 & - & 14 & 2,660 & 7.2 & 36 & - \\
\hline $10-11-00$ & 930 & 2.1 & 14 & 3,400 & 7.1 & 36 & - \\
\hline \multicolumn{8}{|c|}{ Duchesne River near Randlett, Utah (09302000) } \\
\hline $11-05-90$ & 1515 & 74 & 6.0 & 2,300 & 8.2 & - & - \\
\hline $12-10-90$ & 1545 & 150 & 0 & 2,020 & 8.1 & - & - \\
\hline $02-26-91$ & 1200 & 212 & 0 & 1,480 & 8.0 & - & - \\
\hline 03-20-91 & 1550 & 113 & 9.0 & 1,970 & 8.5 & - & - \\
\hline $04-22-91$ & 1330 & 43 & 15.0 & 2,510 & 8.3 & - & - \\
\hline $05-28-91$ & 1330 & 69 & 16.5 & 2,080 & 8.3 & $<1$ & - \\
\hline
\end{tabular}


Table 5. Discharge, physical properties, and selenium concentration of water from the Green River and selected tributaries, Utah and Colorado, water years 1991-2000—Continued

\begin{tabular}{|c|c|c|c|c|c|c|c|}
\hline Date & Time & $\begin{array}{l}\text { Discharge, } \\
\text { instant- } \\
\text { aneous } \\
\left(\mathrm{ft}^{3} / \mathrm{s}\right)\end{array}$ & $\begin{array}{l}\text { Temper- } \\
\text { ature } \\
\left({ }^{\circ} \mathrm{C}\right)\end{array}$ & $\begin{array}{c}\text { Specific } \\
\text { conduc- } \\
\text { tance } \\
(\mu \mathrm{S} / \mathrm{cm})\end{array}$ & $\begin{array}{c}\text { pH, water, } \\
\text { whole, field } \\
\text { (standard units) }\end{array}$ & $\begin{array}{c}\text { Selenium, } \\
\text { dissolved } \\
(\mu \mathrm{g} / \mathrm{L} \text { as } \mathrm{Se})\end{array}$ & $\begin{array}{c}\text { Selenium, } \\
\text { total } \\
(\mu \mathrm{g} / \mathrm{L} \text { as } \mathrm{Se})\end{array}$ \\
\hline \multicolumn{8}{|c|}{ Duchesne River near Randlett, Utah (09302000)—Continued } \\
\hline 06-17-91 & 1430 & 132 & 21.0 & 860 & 8.2 & $<1$ & - \\
\hline 07-19-91 & 1015 & 68 & 21.0 & 1,920 & 8.3 & - & - \\
\hline 08-26-91 & 1745 & 108 & 23.5 & 1,560 & 8.3 & $<1$ & - \\
\hline 09-30-91 & 1520 & 98 & 18.5 & 1,800 & 8.3 & $<1$ & - \\
\hline 11-04-91 & 1400 & 90 & 2.5 & 2,420 & 8.4 & 1.0 & - \\
\hline $12-02-91$ & 1400 & 101 & 0 & 2,310 & 8.2 & $<2$ & - \\
\hline 01-06-92 & 1400 & 208 & 0 & 1,270 & 8.1 & 1.0 & - \\
\hline 02-03-92 & 1630 & 192 & 0 & 1,070 & 7.8 & $<1$ & - \\
\hline 03-06-92 & 1030 & 178 & 4.0 & 1,760 & 8.4 & $<1$ & - \\
\hline 04-06-92 & 1600 & 64 & 16.0 & 2,020 & 8.3 & $<1$ & - \\
\hline 05-04-92 & 1510 & 61 & 22.0 & 2,230 & 8.3 & 1.0 & - \\
\hline 06-01-92 & 1415 & 115 & 22.0 & 1,800 & 8.4 & $<1$ & - \\
\hline 07-06-92 & 1415 & 64 & 23.5 & 1,930 & 8.4 & $<1$ & - \\
\hline 08-03-92 & 1400 & 65 & 25.5 & 1,890 & 8.4 & $<1$ & - \\
\hline $08-31-92$ & 1410 & 74 & 19.5 & 1,960 & 8.3 & $<1$ & - \\
\hline $10-05-92$ & 1300 & 63 & 14.0 & 2,060 & 8.3 & $<1$ & - \\
\hline $11-02-92$ & 1430 & 92 & 10.0 & 2,840 & 8.5 & 1.0 & - \\
\hline $12-07-92$ & 1645 & 81 & -.5 & 2,110 & 8.5 & 1.0 & - \\
\hline 01-04-93 & 1600 & 88 & -.5 & - & 8.2 & - & - \\
\hline 03-08-93 & 1330 & 146 & 0 & 1,000 & 8.3 & $<1$ & - \\
\hline $04-12-93$ & 1630 & 126 & 7.0 & 1,980 & 8.5 & $<1$ & - \\
\hline $05-14-93$ & 1100 & 41 & 16.0 & 2,400 & 8.3 & $<1$ & - \\
\hline $06-15-93$ & 1730 & 384 & 23.0 & 980 & 8.2 & $<1$ & - \\
\hline $07-16-93$ & 1100 & 100 & 19.0 & 1,520 & 8.3 & $<1$ & - \\
\hline $08-16-93$ & 1500 & 94 & 21.0 & 1,790 & 8.5 & $<1$ & - \\
\hline $09-13-93$ & 1630 & 67 & 17.0 & 1,890 & 8.6 & 1.0 & - \\
\hline $10-04-93$ & 1715 & 53 & 16.0 & 2,030 & 8.4 & $<1$ & - \\
\hline $11-01-93$ & 1430 & 275 & 6.5 & 1,250 & 8.6 & $<1$ & - \\
\hline $12-06-93$ & 1650 & 315 & 0 & 1,020 & 8.4 & $<1$ & - \\
\hline 01-10-94 & 1215 & 200 & 0 & 1,200 & 8.7 & $<1$ & - \\
\hline 02-14-94 & 1540 & 215 & 0 & 1,080 & 8.5 & $<1$ & - \\
\hline 03-14-94 & 1400 & 154 & 11.0 & 1,260 & 8.5 & $<1$ & $<1$ \\
\hline $04-25-94$ & 1300 & 66 & 15.0 & 2,130 & 8.4 & $<1$ & - \\
\hline $05-17-94$ & 0915 & 43 & 15.0 & 2,090 & 8.4 & $<1$ & $<1$ \\
\hline $06-22-94$ & 1145 & 58 & 25.0 & 1,960 & 8.4 & $<1$ & $<1$ \\
\hline $07-27-94$ & 0920 & 13 & 24.0 & 2,290 & 8.5 & $<1$ & - \\
\hline $07-12-95$ & 1400 & 4,760 & 18.5 & 240 & 8.2 & $<1$ & - \\
\hline $08-28-95$ & 1355 & 203 & 24.5 & 1,350 & 8.4 & $<2$ & - \\
\hline $10-03-95$ & 1300 & 233 & 12.0 & 1,380 & 8.4 & $<1$ & - \\
\hline $12-11-95$ & 1400 & 390 & 3.0 & 890 & 8.4 & - & - \\
\hline 04-02-96 & 1400 & 388 & 10.0 & 900 & 8.4 & $<1$ & - \\
\hline $05-15-96$ & 1315 & 544 & 19.0 & 870 & 8.3 & $<1$ & - \\
\hline
\end{tabular}


Table 5. Discharge, physical properties, and selenium concentration of water from the Green River and selected tributaries, Utah and Colorado, water years 1991-2000-Continued

\begin{tabular}{|c|c|c|c|c|c|c|c|}
\hline Date & Time & $\begin{array}{l}\text { Discharge, } \\
\text { instant- } \\
\text { aneous } \\
\left(\mathrm{ft}^{3} / \mathrm{s}\right)\end{array}$ & $\begin{array}{l}\text { Temper- } \\
\text { ature } \\
\left({ }^{\circ} \mathrm{C}\right)\end{array}$ & $\begin{array}{c}\text { Specific } \\
\text { conduc- } \\
\text { tance } \\
(\mu \mathrm{S} / \mathrm{cm})\end{array}$ & $\begin{array}{c}\mathrm{pH}, \text { water, } \\
\text { whole, field } \\
\text { (standard units) }\end{array}$ & $\begin{array}{c}\text { Selenium, } \\
\text { dissolved } \\
(\mu \mathrm{g} / \mathrm{L} \text { as } \mathrm{Se})\end{array}$ & $\begin{array}{c}\text { Selenium, } \\
\text { total } \\
(\mu \mathrm{g} / \mathrm{L} \text { as } \mathrm{Se})\end{array}$ \\
\hline \multicolumn{8}{|c|}{ Duchesne River near Randlett, Utah (09302000)_Continued } \\
\hline $07-09-96$ & 1315 & 121 & 24.0 & 1,390 & 8.4 & $<1$ & - \\
\hline $08-20-96$ & 1330 & 89 & 23.0 & 1,410 & 8.4 & $<1$ & - \\
\hline $10-16-96$ & 1245 & 61 & 12.0 & 1,870 & 8.4 & $<1$ & - \\
\hline $12-06-96$ & 1245 & 283 & 0 & 1,340 & 8.5 & $<1$ & - \\
\hline 03-07-97 & 1430 & 515 & 1.0 & 830 & 8.2 & $<1$ & - \\
\hline 04-16-97 & 1200 & 606 & 10.0 & 930 & 8.4 & $<1$ & - \\
\hline $05-20-97$ & 1200 & 2,490 & 15.0 & 330 & 8.8 & $<1$ & - \\
\hline $06-25-97$ & 1330 & 800 & 17.5 & 470 & 8.2 & $<1$ & - \\
\hline 08-05-97 & 1600 & 1,100 & 22.0 & 1,190 & 8.0 & 1.6 & - \\
\hline $10-10-97$ & 1300 & 1,420 & 11.5 & 530 & 8.5 & $<1$ & - \\
\hline $11-21-97$ & 1245 & 1,000 & 3.0 & 650 & 8.5 & $<1$ & - \\
\hline 01-08-98 & 1400 & 650 & 0 & 680 & 8.5 & $<1$ & - \\
\hline $02-25-98$ & 1415 & 851 & 4.0 & 880 & 8.6 & $<1$ & - \\
\hline 04-15-98 & 1315 & 616 & 7.0 & 750 & 8.6 & $<1$ & - \\
\hline $05-20-98$ & 1100 & 497 & 18.0 & 810 & 8.3 & $<1$ & - \\
\hline 07-09-98 & 1345 & 1,200 & 18.0 & 420 & 8.2 & $<1$ & - \\
\hline 08-19-98 & 1645 & 517 & 18.0 & 890 & 8.4 & $<1$ & - \\
\hline $10-14-98$ & 1645 & 718 & 10.0 & 800 & 8.0 & $<1$ & - \\
\hline $12-01-98$ & 1520 & 892 & 5.5 & 630 & 7.5 & 1.0 & - \\
\hline 01-14-99 & 1030 & - & 0 & 670 & 8.0 & $<1$ & - \\
\hline 02-17-99 & 1400 & 860 & 3.5 & 690 & 8.5 & $<1$ & - \\
\hline 03-24-99 & 1400 & 605 & 11.0 & 630 & 8.6 & $<1$ & - \\
\hline 05-11-99 & 1610 & 238 & 14.0 & 1,240 & 8.6 & $<1$ & - \\
\hline 06-11-99 & 1140 & 2,970 & 13.0 & 325 & 8.3 & $<1$ & - \\
\hline 07-21-99 & 1700 & 425 & 24.0 & 860 & 8.6 & $<1$ & - \\
\hline 08-27-99 & 1440 & 411 & 22.0 & 810 & 8.4 & $<1$ & - \\
\hline $10-13-99$ & 1400 & 427 & 14.0 & 870 & 8.7 & $<2.4$ & - \\
\hline $11-30-99$ & 1500 & 490 & 2.0 & 810 & 8.8 & $<2.4$ & - \\
\hline $01-11-00$ & 1540 & 507 & 0 & 670 & 8.4 & $<2.4$ & - \\
\hline 02-29-00 & 1530 & 301 & 7.5 & 970 & 8.8 & $<2.4$ & - \\
\hline 04-11-00 & 1540 & 300 & 15.5 & 820 & 8.8 & $1.3 \mathrm{E}$ & - \\
\hline $05-24-00$ & 1600 & 102 & 21.5 & 1,430 & 8.5 & 4.0 & - \\
\hline $07-11-00$ & 1110 & - & 23.0 & 1,560 & 8.3 & $<2.4$ & - \\
\hline 09-01-00 & 1230 & - & 21.0 & 1,750 & 8.3 & $<2.4$ & - \\
\hline \multicolumn{8}{|c|}{ White River near Watson, Utah (09306500) } \\
\hline $11-07-90$ & 1120 & 430 & 3.5 & 770 & 8.2 & - & - \\
\hline $12-11-90$ & 1130 & 249 & 0 & 870 & 7.7 & - & - \\
\hline 03-21-91 & 1130 & 388 & 2.5 & 880 & 8.3 & - & - \\
\hline $04-25-91$ & 1030 & 370 & 10.5 & 860 & 8.5 & - & - \\
\hline $05-30-91$ & 1700 & 1,930 & 12.5 & 355 & 8.2 & $<1$ & - \\
\hline 06-20-91 & 1200 & 1,690 & 16.5 & 340 & 8.3 & - & - \\
\hline $07-17-91$ & 1100 & 431 & 23.5 & 640 & 8.5 & 1.0 & - \\
\hline
\end{tabular}


Table 5. Discharge, physical properties, and selenium concentration of water from the Green River and selected tributaries, Utah and Colorado, water years 1991-2000—Continued

\begin{tabular}{|c|c|c|c|c|c|c|c|}
\hline Date & Time & $\begin{array}{l}\text { Discharge, } \\
\text { instant- } \\
\text { aneous } \\
\left(\mathrm{ft}^{3} / \mathrm{s}\right)\end{array}$ & $\begin{array}{l}\text { Temper- } \\
\text { ature } \\
\left({ }^{\circ} \mathrm{C}\right)\end{array}$ & $\begin{array}{c}\text { Specific } \\
\text { conduc- } \\
\text { tance } \\
(\mu \mathrm{S} / \mathrm{cm})\end{array}$ & $\begin{array}{c}\mathrm{pH}, \text { water, } \\
\text { whole, field } \\
\text { (standard units) }\end{array}$ & $\begin{array}{c}\text { Selenium, } \\
\text { dissolved } \\
(\mu \mathrm{g} / \mathrm{L} \text { as } \mathrm{Se})\end{array}$ & $\begin{array}{l}\text { Selenium, } \\
\text { total } \\
(\mu g / L \text { as Se) }\end{array}$ \\
\hline \multicolumn{8}{|c|}{ White River near Watson, Utah $(09306500)$ C Continued } \\
\hline $09-10-91$ & 1145 & 333 & 19.0 & 720 & 8.3 & 2.0 & - \\
\hline $10-03-91$ & 1100 & 329 & 13.5 & 730 & 8.4 & - & - \\
\hline $11-06-91$ & 1200 & 325 & 4.5 & 760 & 8.3 & $<1$ & - \\
\hline $12-05-91$ & 1130 & 211 & 0 & 890 & 8.3 & $<1$ & - \\
\hline 01-07-92 & 1130 & 300 & 0 & 850 & 8.2 & 2.0 & - \\
\hline 02-05-92 & 1145 & 400 & 0 & 380 & 8.0 & $<1$ & - \\
\hline 03-04-92 & 1300 & 178 & 2.0 & 810 & 8.2 & 1.0 & - \\
\hline 04-08-92 & 1245 & 436 & 12.5 & 880 & 8.3 & $<1$ & - \\
\hline $05-05-92$ & 1145 & 1,260 & 16.5 & 485 & 8.2 & 1.0 & - \\
\hline 06-03-92 & 1230 & 961 & 19.0 & 455 & 8.5 & $<1$ & - \\
\hline 07-07-92 & 1115 & 394 & 19.5 & 670 & 8.4 & $<1$ & - \\
\hline 08-05-92 & 1620 & 307 & 25.5 & 700 & 8.4 & $<1$ & - \\
\hline 09-01-92 & 1130 & 246 & - & 760 & 8.4 & $<1$ & - \\
\hline $10-07-92$ & 1130 & 272 & 10.5 & 810 & 8.5 & 1.0 & - \\
\hline $11-05-92$ & 1230 & 429 & 7.5 & 776 & 8.5 & 1.0 & - \\
\hline $12-09-92$ & 1315 & 308 & .5 & 820 & 8.6 & 1.0 & - \\
\hline 01-06-93 & 1100 & 253 & 0 & 810 & 8.4 & $<1$ & - \\
\hline $03-11-93$ & 1030 & 600 & 1.0 & 800 & 8.3 & 1.0 & - \\
\hline $04-12-93$ & 1300 & 743 & 8.0 & 1,140 & 8.5 & 4.0 & - \\
\hline $05-11-93$ & 1330 & 1,120 & 16.0 & 740 & 8.6 & 2.0 & - \\
\hline $06-02-93$ & 1320 & 3,720 & 13.0 & 420 & 8.1 & 1.0 & - \\
\hline $07-12-93$ & 1830 & 982 & 22.0 & 470 & 8.4 & $<1$ & - \\
\hline 08-17-93 & 1100 & 558 & 19.0 & 750 & 8.6 & 1.0 & - \\
\hline $09-15-93$ & 1200 & 265 & 15.0 & 780 & 8.5 & 1.0 & - \\
\hline $10-05-93$ & 1130 & 265 & 13.0 & 760 & 8.5 & 1.0 & - \\
\hline $11-03-93$ & 1200 & 468 & 5.0 & 760 & 8.4 & 1.0 & - \\
\hline $12-07-93$ & 1330 & 506 & 0 & 820 & 8.7 & 1.0 & - \\
\hline 01-11-94 & 0915 & 295 & -.5 & 850 & 8.7 & 1.0 & - \\
\hline $03-18-94$ & 0930 & 502 & 6.0 & 870 & 8.6 & 1.0 & - \\
\hline $04-26-94$ & 0900 & 752 & 11.5 & 735 & 8.5 & 1.0 & - \\
\hline $05-19-94$ & 1045 & 1,230 & 15.5 & 470 & 8.4 & $<1$ & - \\
\hline 06-24-94 & 0915 & 397 & 21.5 & 580 & 8.5 & $<1$ & - \\
\hline 07-28-94 & 1730 & 209 & 27.0 & 765 & 8.5 & $<1$ & - \\
\hline $08-25-94$ & 1015 & 145 & 23.0 & 860 & 8.6 & $<1$ & - \\
\hline $10-17-94$ & 1600 & 399 & 9.5 & 780 & 8.6 & $<1$ & - \\
\hline $11-30-94$ & 0915 & 153 & .5 & 820 & 8.4 & - & - \\
\hline 04-06-95 & 0845 & 350 & 9.0 & 820 & 8.4 & 1.0 & - \\
\hline $05-17-95$ & 0935 & 1,780 & 13.0 & 720 & 8.4 & 3.0 & - \\
\hline 07-14-95 & 0940 & 3,510 & 17.0 & 390 & 8.3 & $<1$ & - \\
\hline 08-30-95 & 0845 & 495 & 19.0 & 660 & 8.4 & 1.0 & - \\
\hline $10-05-95$ & 0815 & 632 & 9.5 & 690 & 8.4 & $<1$ & - \\
\hline $12-13-95$ & 1030 & 465 & 3.5 & 750 & 8.4 & - & - \\
\hline
\end{tabular}


Table 5. Discharge, physical properties, and selenium concentration of water from the Green River and selected tributaries, Utah and Colorado, water years 1991-2000-Continued

\begin{tabular}{|c|c|c|c|c|c|c|c|}
\hline Date & Time & $\begin{array}{l}\text { Discharge, } \\
\text { instant- } \\
\text { aneous } \\
\left(\mathrm{ft}^{3} / \mathrm{s}\right)\end{array}$ & $\begin{array}{c}\text { Temper- } \\
\text { ature } \\
\left({ }^{\circ} \mathrm{C}\right)\end{array}$ & $\begin{array}{c}\text { Specific } \\
\text { conduc- } \\
\text { tance } \\
(\mu \mathrm{S} / \mathrm{cm})\end{array}$ & $\begin{array}{c}\mathrm{pH}, \text { water, } \\
\text { whole, field } \\
\text { (standard units) }\end{array}$ & $\begin{array}{l}\text { Selenium, } \\
\text { dissolved } \\
(\mu \mathrm{g} / \mathrm{L} \text { as } \mathrm{Se})\end{array}$ & $\begin{array}{l}\text { Selenium, } \\
\text { total } \\
(\mu \mathrm{g} / \mathrm{L} \text { as Se })\end{array}$ \\
\hline \multicolumn{8}{|c|}{ White River near Watson, Utah (09306500)_Continued } \\
\hline 04-04-96 & 0930 & 622 & 8.5 & 870 & 8.6 & 2.0 & - \\
\hline $05-17-96$ & 0935 & 2,830 & 13.5 & 350 & 8.3 & $<1$ & - \\
\hline $06-06-96$ & 0900 & 747 & 21.0 & 520 & 8.5 & $<1$ & - \\
\hline $08-22-96$ & 0900 & 359 & 20.5 & 690 & 8.5 & $<1$ & - \\
\hline $10-16-96$ & 0930 & 410 & 10.5 & 680 & 8.5 & $<1$ & - \\
\hline $12-05-96$ & 1530 & 364 & .5 & 730 & 8.6 & 1.0 & - \\
\hline $11-19-97$ & 1315 & 717 & 3.5 & 700 & 8.5 & 1.3 & - \\
\hline 04-16-98 & 1200 & 1,160 & 7.2 & 940 & 8.7 & 3.2 & - \\
\hline 07-08-98 & 1145 & 1,340 & 20.5 & 490 & 8.3 & - & - \\
\hline $10-15-98$ & 1130 & 607 & 12.0 & 750 & 7.8 & $<1$ & - \\
\hline 03-25-99 & 1210 & 549 & 12.0 & 850 & 8.6 & 1.5 & - \\
\hline 08-26-99 & 1210 & 438 & 23.5 & 700 & 8.4 & 1.3 & - \\
\hline 10-14-99 & 0920 & 387 & 10.0 & 750 & 8.6 & $1.3 \mathrm{E}$ & - \\
\hline $07-12-00$ & 1850 & 322 & 25.0 & 700 & 8.3 & $<2.4$ & - \\
\hline $08-31-00$ & 1130 & 349 & 21.0 & 840 & 8.5 & $<2.4$ & - \\
\hline \multicolumn{8}{|c|}{ Pariette Draw at mouth near Ouray, Utah (09307300) } \\
\hline $06-21-91$ & 1345 & 20 & 23.0 & 2,450 & 8.8 & 5.0 & - \\
\hline $08-26-91$ & 1430 & 1.3 & 21.5 & 3,860 & 7.8 & 2.0 & - \\
\hline \multicolumn{8}{|c|}{ Price River at Woodside, Utah $(09314500)$} \\
\hline $10-10-90$ & 0750 & 22 & 5.5 & 3,700 & - & - & - \\
\hline $11-26-90$ & 1000 & 12 & .5 & 4,380 & - & - & - \\
\hline $12-20-90$ & 0940 & 22 & 0 & 4,080 & - & - & - \\
\hline $01-25-91$ & 0940 & 9.1 & 0 & 2,850 & - & - & - \\
\hline $02-22-91$ & 1000 & 24 & 0 & 1,550 & - & - & - \\
\hline 03-29-91 & 0950 & 21 & 6.0 & 3,980 & - & - & - \\
\hline $04-25-91$ & 1215 & 14 & 12.5 & 4,450 & - & - & - \\
\hline $05-23-91$ & 0930 & 17 & 15.5 & 4,320 & - & - & - \\
\hline $06-24-91$ & 1040 & 16 & 18.0 & 2,200 & 8.3 & - & - \\
\hline 07-26-91 & 1000 & 23 & 18.0 & 2,320 & 8.0 & 3.0 & - \\
\hline 08-29-91 & 1000 & 25 & 23.0 & 850 & 7.8 & 1.0 & - \\
\hline $11-25-91$ & 1030 & 43 & 0 & 4,200 & 8.3 & $<1$ & - \\
\hline 03-10-92 & 1000 & 64 & 6.0 & 2,340 & 8.4 & 2.0 & - \\
\hline 04-22-92 & 0930 & 21 & 14.0 & 3,120 & 8.3 & 2.0 & - \\
\hline $05-29-92$ & 0945 & 85 & 17.0 & 1,780 & 8.0 & 3.0 & - \\
\hline 06-19-92 & 0945 & 6.7 & 18.5 & 3,750 & 8.2 & 2.0 & - \\
\hline $07-20-92$ & 0945 & 4.8 & 22.0 & 3,250 & 8.3 & 3.0 & - \\
\hline $11-16-92$ & 0940 & 11 & 2.0 & 4,750 & 8.4 & 3.0 & - \\
\hline $02-25-93$ & 0940 & 44 & 2.0 & 1,810 & 8.5 & 3.0 & - \\
\hline $03-26-93$ & 1300 & 107 & 13.0 & 2,440 & 8.3 & 7.0 & - \\
\hline $05-21-93$ & 1030 & 800 & 17.0 & 900 & 8.4 & 2.0 & - \\
\hline $06-23-93$ & 1040 & 40 & 20.0 & 2,600 & 8.4 & 2.0 & - \\
\hline $11-16-93$ & 1345 & 51 & 3.5 & 4,000 & 8.5 & 3.0 & - \\
\hline
\end{tabular}


Table 5. Discharge, physical properties, and selenium concentration of water from the Green River and selected tributaries, Utah and Colorado, water years 1991-2000—Continued

\begin{tabular}{|c|c|c|c|c|c|c|c|}
\hline Date & Time & $\begin{array}{l}\text { Discharge, } \\
\text { instant- } \\
\text { aneous } \\
\left(\mathrm{ft}^{3} / \mathrm{s}\right)\end{array}$ & $\begin{array}{l}\text { Temper- } \\
\text { ature } \\
\left({ }^{\circ} \mathrm{C}\right)\end{array}$ & $\begin{array}{c}\text { Specific } \\
\text { conduc- } \\
\text { tance } \\
(\mu \mathrm{S} / \mathrm{cm})\end{array}$ & $\begin{array}{c}\text { pH, water, } \\
\text { whole, field } \\
\text { (standard units) }\end{array}$ & $\begin{array}{c}\text { Selenium, } \\
\text { dissolved } \\
(\mu \mathrm{g} / \mathrm{L} \text { as } \mathrm{Se})\end{array}$ & $\begin{array}{c}\text { Selenium, } \\
\text { total } \\
(\mu \mathrm{g} / \mathrm{L} \text { as Se) }\end{array}$ \\
\hline \multicolumn{8}{|c|}{ Price River at Woodside, Utah $(\mathbf{0 9 3 1 4 5 0 0 )}$ —Continued } \\
\hline $04-19-94$ & 0945 & 23 & 14.5 & 4,530 & 8.2 & 2.0 & - \\
\hline $05-25-94$ & 1200 & 17 & 23.0 & 3,420 & 8.4 & 2.0 & - \\
\hline $06-23-94$ & 1200 & 9.2 & 32.0 & 3,100 & 8.3 & $<1$ & - \\
\hline $07-19-94$ & 1315 & 5.1 & 25.0 & 2,980 & 8.3 & 1.0 & - \\
\hline $03-27-95$ & 0955 & 74 & 4.5 & 2,050 & 8.4 & 3.0 & - \\
\hline $05-31-95$ & 0910 & 350 & 14.0 & 1,300 & 8.2 & 2.0 & - \\
\hline $06-30-95$ & 1315 & 400 & 18.5 & 1,150 & 8.4 & $<1$ & - \\
\hline $07-24-95$ & 1245 & 120 & 20.5 & 1,890 & 8.4 & 1.0 & - \\
\hline 08-17-95 & 1330 & 170 & 21.5 & 1,490 & 8.3 & $<2$ & - \\
\hline $10-30-95$ & 1000 & 93 & 9.5 & 2,590 & 8.4 & 2.0 & - \\
\hline $03-20-96$ & 0930 & 171 & 6.0 & 1,600 & 8.4 & 1.0 & - \\
\hline 04-24-96 & 1000 & 400 & 10.5 & 1,250 & 8.4 & 1.0 & - \\
\hline 06-06-96 & 0910 & 250 & 19.5 & 2,740 & 8.3 & 2 & - \\
\hline $06-27-96$ & 1300 & 54 & 19.5 & 3,520 & 8.4 & $<1$ & - \\
\hline $11-22-96$ & 0930 & 67 & 7.0 & 3,100 & 8.2 & 2.0 & - \\
\hline 04-08-97 & 0920 & 225 & 7.0 & 1,830 & 8.5 & 2.0 & - \\
\hline 06-03-97 & 1200 & 600 & 19.0 & 1,530 & 8.4 & 1.9 & - \\
\hline 08-14-97 & 1130 & 350 & 19.5 & 1,650 & 8.3 & 2.0 & - \\
\hline 09-05-97 & 1215 & 400 & 20.0 & 2,040 & 8.2 & 2.3 & - \\
\hline \multicolumn{8}{|c|}{ Green River at Green River, Utah (09315000) } \\
\hline $10-17-90$ & 1230 & 2,240 & 11.0 & 960 & 8.4 & - & - \\
\hline $11-19-90$ & 1200 & 2,060 & 6.0 & 910 & 8.3 & $<3$ & - \\
\hline $03-25-91$ & 0930 & 2,390 & 8.0 & 980 & 8.4 & 2.0 & - \\
\hline $04-22-91$ & 1030 & 2,610 & 13.0 & 840 & 8.4 & 2.0 & - \\
\hline $05-22-91$ & 1030 & 8,660 & 16.0 & 390 & 8.1 & - & - \\
\hline $06-25-91$ & 1100 & 6,510 & 20.5 & 425 & 8.2 & $<1$ & - \\
\hline $07-24-91$ & 1045 & 2,600 & 23.0 & 760 & 8.4 & $<1$ & - \\
\hline $08-26-91$ & 1100 & 2,170 & 23.5 & 790 & 8.3 & $<1$ & - \\
\hline $10-01-91$ & 1030 & 2,160 & 17.0 & 870 & 8.4 & - & - \\
\hline $11-21-91$ & 1100 & 3,020 & 4.0 & 940 & 8.4 & 2.0 & - \\
\hline $03-25-92$ & 1045 & 3,190 & 9.0 & 880 & 8.4 & 2.0 & - \\
\hline $04-21-92$ & 1030 & 4,850 & 12.5 & 530 & 8.4 & $<1$ & - \\
\hline $05-28-92$ & 1115 & 7,490 & 20.0 & 430 & 8.2 & $<1$ & - \\
\hline $06-23-92$ & 1030 & 2,720 & 23.0 & 520 & 8.3 & $<1$ & - \\
\hline $07-21-92$ & 1000 & 2,590 & 19.0 & 790 & 8.4 & 1.0 & - \\
\hline $08-25-92$ & 1045 & 1,860 & 20.5 & 990 & 8.2 & - & - \\
\hline $10-13-92$ & 1100 & 1,550 & 13.5 & 860 & 8.2 & - & - \\
\hline $11-24-92$ & 1030 & 2,090 & 2.5 & 930 & 8.2 & 1.0 & - \\
\hline $11-24-92$ & 1035 & 2,090 & 2.5 & 930 & 8.2 & 2.0 & - \\
\hline $02-23-93$ & 1230 & 2,050 & 2.0 & 900 & 8.2 & - & - \\
\hline $03-25-93$ & 1000 & 4,910 & 9.5 & 900 & 8.3 & 3.0 & - \\
\hline $03-25-93$ & 1005 & 4,910 & 9.5 & 900 & 8.3 & 3.0 & - \\
\hline
\end{tabular}


Table 5. Discharge, physical properties, and selenium concentration of water from the Green River and selected tributaries, Utah and Colorado, water years 1991-2000-Continued

\begin{tabular}{|c|c|c|c|c|c|c|c|}
\hline Date & Time & $\begin{array}{l}\text { Discharge, } \\
\text { instant- } \\
\text { aneous } \\
\left(\mathrm{ft}^{3} / \mathrm{s}\right)\end{array}$ & $\begin{array}{c}\text { Temper- } \\
\text { ature } \\
\left({ }^{\circ} \mathrm{C}\right)\end{array}$ & $\begin{array}{c}\text { Specific } \\
\text { conduc- } \\
\text { tance } \\
(\mu \mathrm{S} / \mathrm{cm})\end{array}$ & $\begin{array}{c}\text { pH, water, } \\
\text { whole, field } \\
\text { (standard units) }\end{array}$ & $\begin{array}{l}\text { Selenium, } \\
\text { dissolved } \\
(\mu \mathrm{g} / \mathrm{L} \text { as Se })\end{array}$ & $\begin{array}{l}\text { Selenium, } \\
\text { total } \\
(\mu \mathrm{g} / \mathrm{L} \text { as Se })\end{array}$ \\
\hline \multicolumn{8}{|c|}{ Green River at Green River, Utah $(09315000)$-Continued } \\
\hline $04-29-93$ & 1100 & 5,100 & 15.0 & 870 & 8.5 & 4.0 & - \\
\hline $05-20-93$ & 1105 & 21,200 & 17.0 & 420 & 8.0 & 1.0 & - \\
\hline $06-21-93$ & 1100 & 17,500 & 19.5 & 360 & 8.0 & $<1$ & - \\
\hline $06-21-93$ & 1105 & 17,500 & 19.5 & 360 & 8.0 & $<1$ & - \\
\hline $07-20-93$ & 1230 & 3,910 & 24.0 & 475 & 8.2 & - & - \\
\hline $08-26-93$ & 1130 & 2,240 & 22.0 & 850 & 8.4 & - & - \\
\hline $10-05-93$ & 1130 & 1,950 & 16.0 & 880 & 8.5 & - & - \\
\hline 03-29-94 & 1115 & 3,170 & 9.0 & 870 & 8.4 & 5.0 & - \\
\hline $04-25-94$ & 1110 & 5,620 & 14.0 & 750 & 8.3 & 1.0 & - \\
\hline $05-20-94$ & 1030 & 11,800 & 15.0 & 420 & 8.1 & $<1$ & - \\
\hline $06-27-94$ & 1030 & 2,500 & 24.0 & 580 & 8.3 & $<1$ & - \\
\hline $07-25-94$ & 1120 & 1,650 & 25.0 & 790 & 8.4 & $<1$ & - \\
\hline 08-23-94 & 1000 & 1,580 & 22.5 & 820 & 8.4 & - & - \\
\hline $10-03-94$ & 1015 & 2,220 & 15.0 & 870 & 8.4 & - & - \\
\hline $11-14-94$ & 1130 & 1,950 & 5.0 & 1,020 & 8.4 & 2.0 & - \\
\hline $03-20-95$ & 1315 & 2,480 & 11.5 & 930 & 8.4 & 3.0 & - \\
\hline $05-22-95$ & 1250 & 14,600 & 17.0 & 465 & 8.2 & 1.0 & - \\
\hline $06-23-95$ & 1130 & 24,600 & 17.5 & 310 & 8.3 & $<1$ & - \\
\hline $07-20-95$ & 1135 & 12,300 & 21.0 & 380 & 8.2 & $<1$ & - \\
\hline $09-28-95$ & 1130 & 2,900 & 15.0 & 900 & 8.5 & 1.0 & - \\
\hline $10-24-95$ & 1200 & 3,300 & 8.0 & 880 & 8.5 & 2.0 & - \\
\hline $11-28-95$ & 1200 & 4,390 & 4.0 & 850 & 8.3 & 1.0 & - \\
\hline $03-15-96$ & 1030 & 5,140 & 8.5 & 840 & 8.3 & 1.0 & - \\
\hline $03-26-96$ & 1200 & 4,910 & 6.0 & 820 & 8.3 & 2.0 & - \\
\hline $04-25-96$ & 1140 & 8,430 & 12.0 & 640 & 8.3 & 2.0 & - \\
\hline 06-04-96 & 1055 & 14,800 & 18.5 & 470 & 8.3 & $<1$ & - \\
\hline 06-12-96 & 1030 & 15,900 & 19.5 & 375 & 8.2 & $<1$ & - \\
\hline $06-25-96$ & 1125 & 14,600 & 19.5 & 450 & 8.3 & $<1$ & - \\
\hline 07-24-96 & 1115 & 3,100 & 25.0 & 670 & 8.4 & 1.0 & - \\
\hline 08-29-96 & 1500 & 2,200 & 23.0 & 800 & 8.5 & $<1$ & - \\
\hline 09-24-96 & 1120 & 2,700 & 16.0 & 930 & 8.5 & 1.0 & - \\
\hline $11-26-96$ & 1145 & 4,050 & 7.0 & 850 & 8.5 & 1.0 & - \\
\hline $02-21-97$ & 1100 & 4,950 & 1.0 & 800 & 8.5 & 1.0 & - \\
\hline 03-18-97 & 1115 & 9,000 & 6.5 & 740 & 8.3 & 1.0 & - \\
\hline 04-29-97 & 1100 & 12,400 & 13.0 & 600 & 8.3 & $<1$ & - \\
\hline 05-19-97 & 1045 & 23,500 & 17.5 & 385 & 8.2 & $<1$ & - \\
\hline $05-27-97$ & 1210 & 27,700 & 16.0 & 385 & 8.2 & $<1$ & - \\
\hline 06-09-97 & 1030 & 32,000 & 19.5 & 350 & 8.2 & $<1$ & - \\
\hline 06-24-97 & 1400 & 23,600 & 22.0 & 395 & 8.3 & $<1$ & - \\
\hline 07-08-97 & 1030 & 9,200 & 22.5 & 480 & 8.5 & $<1$ & - \\
\hline $07-21-97$ & 1700 & 4,710 & 23.0 & 560 & 8.5 & $<1$ & - \\
\hline $08-20-97$ & 1310 & 4,200 & 22.0 & 700 & 8.5 & 1.0 & - \\
\hline
\end{tabular}


Table 5. Discharge, physical properties, and selenium concentration of water from the Green River and selected tributaries, Utah and Colorado, water years 1991-2000—Continued

\begin{tabular}{|c|c|c|c|c|c|c|c|}
\hline Date & Time & $\begin{array}{l}\text { Discharge, } \\
\text { instant- } \\
\text { aneous } \\
\left(\mathrm{ft}^{3} / \mathrm{s}\right)\end{array}$ & $\begin{array}{c}\text { Temper- } \\
\text { ature } \\
\left({ }^{\circ} \mathrm{C}\right)\end{array}$ & $\begin{array}{l}\text { Specific } \\
\text { conduc- } \\
\text { tance } \\
(\mu \mathrm{S} / \mathrm{cm})\end{array}$ & $\begin{array}{c}\mathrm{pH}, \text { water, } \\
\text { whole, field } \\
\text { (standard units) }\end{array}$ & $\begin{array}{l}\text { Selenium, } \\
\text { dissolved } \\
(\mu \mathrm{g} / \mathrm{L} \text { as } \mathrm{Se})\end{array}$ & $\begin{array}{c}\text { Selenium, } \\
\text { total } \\
(\mu \mathrm{g} / \mathrm{L} \text { as } \mathrm{Se})\end{array}$ \\
\hline \multicolumn{8}{|c|}{ Green River at Green River, Utah (09315000)_Continued } \\
\hline $08-20-97$ & 1320 & 4,200 & 22.0 & 700 & 8.5 & $<1$ & - \\
\hline $09-22-97$ & 1145 & 8,930 & 17.0 & 720 & 8.3 & $<1$ & - \\
\hline $11-24-97$ & 1146 & 6,340 & 3.0 & 700 & 8.4 & 1.0 & - \\
\hline 02-18-98 & 1450 & 4,920 & 4.0 & 760 & 8.4 & 2.0 & - \\
\hline 03-19-98 & 1230 & 6,390 & 8.5 & 760 & 8.4 & 2.0 & - \\
\hline 04-29-98 & 1300 & 14,300 & 14.0 & 620 & 8.3 & 2.0 & - \\
\hline $05-20-98$ & 1230 & 16,200 & 15.5 & 445 & 8.3 & 1.0 & - \\
\hline 06-01-98 & 1230 & 20,300 & 17.0 & 410 & 8.3 & $<1$ & - \\
\hline $06-23-98$ & 1200 & 18,200 & 17.5 & 450 & 8.3 & $<1$ & - \\
\hline $07-21-98$ & 1205 & 6,090 & 26.0 & 600 & 8.3 & $<1$ & - \\
\hline 08-17-98 & 1240 & 4,120 & 23.5 & 660 & 8.6 & $<1$ & - \\
\hline 09-28-98 & 1230 & 4080 & 17.0 & 760 & 8.5 & $<1$ & - \\
\hline $10-27-98$ & 1430 & 4780 & 12.0 & 780 & 8.4 & $<1$ & - \\
\hline $11-17-98$ & 1145 & 5,020 & 6.0 & 750 & 8.3 & 2.1 & - \\
\hline $12-07-98$ & 1400 & 4,780 & 3.0 & 730 & 8.3 & $<1$ & - \\
\hline 03-24-99 & 1030 & 6,770 & 10.0 & 740 & 8.4 & 1.2 & - \\
\hline 04-19-99 & 1415 & 7,100 & 12.5 & 720 & 8.5 & $<1$ & - \\
\hline 05-19-99 & 1230 & 14,000 & 13.0 & 540 & 8.2 & $<1$ & - \\
\hline 07-01-99 & 1100 & 13,400 & 21.5 & 405 & 8.2 & $<1$ & - \\
\hline 07-29-99 & 1100 & 4,200 & 24.5 & 730 & 8.3 & 1.6 & - \\
\hline 08-25-99 & 1115 & 3,830 & 23.5 & 750 & 8.4 & $<1$ & - \\
\hline 09-22-99 & 1130 & 3,880 & 17.5 & 780 & 8.4 & 1.4 & - \\
\hline $10-18-99$ & 1130 & 3,460 & 9.5 & 780 & 8.4 & $<2$ & - \\
\hline $11-22-99$ & 1230 & 3,750 & 4.0 & 800 & 8.4 & $2.2 \mathrm{E}$ & - \\
\hline $12-14-99$ & 1330 & 3,870 & 0 & 770 & 8.3 & $<2$ & - \\
\hline $03-22-00$ & 1100 & 3,780 & 5.5 & 780 & 7.3 & $2.0 \mathrm{E}$ & - \\
\hline $04-25-00$ & 1200 & 6,410 & 13.5 & 550 & 8.2 & $1.4 \mathrm{E}$ & - \\
\hline $05-25-00$ & 1100 & 9,450 & 18.5 & 435 & 8.0 & $1.9 \mathrm{E}$ & - \\
\hline $06-29-00$ & 1100 & 3,690 & 22.0 & 500 & 8.4 & $<2.4$ & - \\
\hline $07-24-00$ & 1130 & 1,890 & 24.5 & 740 & 8.5 & - & - \\
\hline $08-21-00$ & 1100 & 1,720 & 23.0 & 760 & 8.4 & $<2.4$ & - \\
\hline 09-11-00 & 0930 & 1,930 & 18.5 & 800 & 8.5 & $<2.4$ & \\
\hline
\end{tabular}


Table 6. Discharge, physical properties, selenium concentration, and depth of water at selected sample locations in Brush Creek drainage near Jensen, Utah, water years 1991-2000

[Data from U.S. Geological Survey; $\mathrm{ft}^{3} / \mathrm{s}$, cubic feet per second; ${ }^{\circ} \mathrm{C}$, degrees Celsius; $\mu \mathrm{S} / \mathrm{cm}$, microsiemens per centimeter at $25^{\circ} \mathrm{C} ; \mu \mathrm{g} / \mathrm{L}$, micrograms per liter; -, no data; <, less than; E, estimated]

\begin{tabular}{|c|c|c|c|c|c|c|c|c|}
\hline Date & Time & $\begin{array}{c}\text { Discharge, } \\
\text { instantaneous } \\
\left(\mathrm{ft}^{3} / \mathrm{s}\right)\end{array}$ & $\begin{array}{c}\text { Temper- } \\
\text { ature } \\
\left({ }^{\circ} \mathrm{C}\right)\end{array}$ & $\begin{array}{c}\text { Specific } \\
\text { conductance } \\
(\mu \mathrm{S} / \mathrm{cm})\end{array}$ & $\begin{array}{l}\text { pH, water, } \\
\text { whole, field } \\
\text { (standard } \\
\text { units) }\end{array}$ & $\begin{array}{c}\text { Selenium, } \\
\text { dissolved } \\
(\mu \mathrm{g} / \mathrm{L} \\
\text { as Se })\end{array}$ & $\begin{array}{c}\text { Selenium, } \\
\text { total } \\
(\mu \mathrm{g} / \mathrm{L} \\
\text { as Se) }\end{array}$ & $\begin{array}{c}\text { Depth at } \\
\text { sample } \\
\text { location, } \\
\text { total } \\
\text { (feet) }\end{array}$ \\
\hline \multicolumn{9}{|c|}{ Big Brush Creek above Red Fleet Reservoir near Vernal, Utah (09261700) } \\
\hline$\overline{05-14-91}$ & 1515 & 149 & 7.0 & 120 & - & - & - & - \\
\hline $05-29-91$ & 1630 & 215 & 8.0 & 86 & - & - & - & - \\
\hline 06-19-91 & 1805 & 76 & 13.0 & 145 & - & - & - & - \\
\hline 07-18-91 & 1610 & 43 & 14.0 & 205 & - & - & - & - \\
\hline $10-26-94$ & 1230 & 25 & 9.0 & 245 & 8.6 & $<1$ & - & - \\
\hline $10-15-99$ & 1215 & 23 & 9.0 & 353 & 8.5 & $<2.4$ & - & - \\
\hline $03-14-00$ & 1715 & - & 8.5 & 393 & 8.5 & $<2.4$ & - & - \\
\hline $05-23-00$ & 1815 & 158 & 9.0 & 93 & 7.8 & $<.7$ & - & - \\
\hline $06-28-00$ & 1415 & - & 12.5 & 190 & 8.4 & $<.7$ & - & - \\
\hline $07-26-00$ & 1410 & 35 & 17.5 & 220 & 8.7 & $<.7$ & - & - \\
\hline $08-30-00$ & 1450 & - & 14.5 & 330 & 8.5 & .8 & - & - \\
\hline \multicolumn{9}{|c|}{ Red Fleet Reservoir near dam (RFR Dam) } \\
\hline $05-18-95$ & 1005 & - & 12.5 & 336 & 8.4 & 1.0 & - & 0 \\
\hline $05-18-95$ & 1030 & - & - & - & - & 1.0 & - & 59.0 \\
\hline $05-18-95$ & 1015 & - & 7.0 & 353 & 7.8 & 1.0 & - & 85.0 \\
\hline \multicolumn{9}{|c|}{ Big Brush Creek below Red Fleet Reservoir (BC1) } \\
\hline $05-19-95$ & 1115 & - & 7.0 & 370 & 8.5 & 1.0 & $<2$ & - \\
\hline \multicolumn{9}{|c|}{ Big Brush Creek at county road near Donkey Flat (BC2) } \\
\hline $10-26-94$ & 1130 & - & 8.5 & 490 & 8.3 & 2.0 & - & - \\
\hline $05-19-95$ & 1100 & - & 9.0 & 390 & 8.4 & 2.0 & - & - \\
\hline 08-14-95 & 1720 & 90 & 14.0 & 185 & - & $<1$ & - & - \\
\hline \multicolumn{9}{|c|}{ Brush Creek below Red Fleet at the corral (BC3) } \\
\hline $10-26-94$ & 1050 & 4.1 & - & 980 & 8.4 & 2.0 & - & - \\
\hline $05-19-95$ & 1045 & - & 9.0 & 520 & - & 2.0 & - & - \\
\hline 08-14-95 & 1700 & - & 15.0 & 205 & - & $<1$ & - & - \\
\hline \multicolumn{9}{|c|}{ Brush Creek upstream of South Fork of Jensen Wash (BC4) } \\
\hline $10-26-94$ & 1020 & .95 & - & 1,100 & 8.2 & 2.0 & - & - \\
\hline 05-19-95 & 1000 & 80 & 8.0 & 420 & 8.5 & 2.0 & - & - \\
\hline \multicolumn{9}{|c|}{ Brush Creek at Sunshine Pipeline Diversion (BC5) } \\
\hline $05-19-95$ & 0950 & - & 8.0 & 440 & 8.4 & 2.0 & - & - \\
\hline 08-14-95 & 1635 & - & 15.0 & 235 & - & $<1$ & - & - \\
\hline $03-26-96$ & 1420 & 5.0 & 6.5 & 660 & 8.3 & 1.0 & - & - \\
\hline $04-17-96$ & 0810 & 5.0 & 9.0 & 440 & 8.1 & $<1$ & - & - \\
\hline $05-16-96$ & 1345 & 102 & 12.5 & 348 & 8.2 & $<1$ & - & - \\
\hline $06-13-96$ & 1400 & 60 & 14.5 & 349 & 8.2 & $<1$ & - & - \\
\hline $10-24-96$ & 1505 & 10 & 5.0 & 530 & 8.2 & 1.0 & - & - \\
\hline \multicolumn{9}{|c|}{ Brush Creek at county road, east of Bullwinkle Reservoir (BC 6) } \\
\hline$\overline{10-26-94}$ & 0950 & - & 6.0 & 1,160 & 8.1 & 3.0 & - & - \\
\hline 05-19-95 & 0940 & - & 8.5 & 445 & 8.1 & 2.0 & - & - \\
\hline
\end{tabular}


Table 6. Discharge, physical properties, selenium concentration, and depth of water at selected sample locations in Brush Creek drainage near Jensen, Utah, water years 1991-2000-Continued

\begin{tabular}{|c|c|c|c|c|c|c|c|c|}
\hline Date & Time & $\begin{array}{c}\text { Discharge, } \\
\text { instantaneous } \\
\left(\mathrm{ft}^{3} / \mathrm{s}\right)\end{array}$ & $\begin{array}{l}\text { Temper- } \\
\text { ature } \\
\left({ }^{\circ} \mathrm{C}\right)\end{array}$ & $\begin{array}{c}\text { Specific } \\
\text { conductance } \\
(\mu \mathrm{S} / \mathrm{cm})\end{array}$ & $\begin{array}{l}\text { pH, water, } \\
\text { whole, field } \\
\text { (standard } \\
\text { units) }\end{array}$ & $\begin{array}{c}\text { Selenium, } \\
\text { dissolved } \\
(\mu \mathrm{g} / \mathrm{L} \\
\text { as Se) }\end{array}$ & $\begin{array}{c}\text { Selenium, } \\
\text { total } \\
(\mu \mathrm{g} / \mathrm{L} \\
\text { as Se) }\end{array}$ & $\begin{array}{c}\text { Depth at } \\
\text { sample } \\
\text { location, } \\
\text { total } \\
\text { (feet) }\end{array}$ \\
\hline \multicolumn{9}{|c|}{ Brush Creek at old diversion for Sunshine Canal (BC 7) } \\
\hline $10-25-94$ & 1545 & 5.5 & 11.5 & 1,150 & 8.4 & 3.0 & - & - \\
\hline $05-19-95$ & 0920 & - & 9.0 & 480 & 8.5 & $<1$ & - & - \\
\hline 08-14-95 & 1610 & - & 15.0 & 250 & - & $<1$ & - & - \\
\hline $03-26-96$ & 1400 & 5.0 & 7.5 & 700 & 8.3 & 1.0 & - & - \\
\hline $04-17-96$ & 0825 & 5.0 & 9.5 & 480 & 8.2 & $<1$ & - & - \\
\hline $05-16-96$ & 1325 & 102 & 12.5 & 355 & 8.2 & $<1$ & - & - \\
\hline $06-13-96$ & 1340 & 60 & 16.5 & 371 & 8.2 & $<1$ & - & - \\
\hline $10-24-96$ & 1445 & 10 & 5.0 & 610 & 8.0 & $<1$ & - & - \\
\hline \multicolumn{9}{|c|}{ Burns Bench Canal below Sunshine Pipeline Crossing (BBC) } \\
\hline $03-15-95$ & 1300 & .01 & 7.5 & 4,050 & - & 150 & - & - \\
\hline \multicolumn{9}{|c|}{ Brush Creek at Burns Bench Canal diversion structure (BC BBC) } \\
\hline $10-25-94$ & 1430 & 3.7 & 9.5 & 1,220 & 8.3 & 3.0 & - & - \\
\hline $05-19-95$ & 0840 & - & 9.5 & 490 & - & 2.0 & 4.0 & - \\
\hline 08-14-95 & 1605 & - & 16.0 & 290 & - & $<1$ & - & - \\
\hline $03-26-96$ & 1330 & 5.0 & 6.0 & 780 & 8.3 & 2.0 & - & - \\
\hline $04-17-96$ & 0840 & 5.0 & 9.0 & 520 & 8.2 & 1.0 & - & - \\
\hline $05-16-96$ & 1310 & 102 & 14.0 & 390 & 8.2 & $<1$ & - & - \\
\hline 06-13-96 & 1330 & 60 & 17.5 & 394 & 8.1 & $<1$ & - & - \\
\hline $10-24-96$ & 1430 & 10 & 4.5 & 640 & 8.1 & 1.1 & - & - \\
\hline 07-13-99 & 1300 & - & 17.0 & 297 & 8.2 & $<1$ & - & - \\
\hline 08-18-99 & 1245 & - & 17.0 & 290 & 8.2 & $<1$ & - & - \\
\hline 09-16-99 & 1200 & - & 13.5 & 436 & 8.2 & $<2.4$ & - & - \\
\hline $10-15-99$ & 1100 & - & 8.5 & 456 & 8.2 & $<2.4$ & - & - \\
\hline $03-14-00$ & 1400 & - & 7.0 & 540 & 8.4 & $2.1 \mathrm{E}$ & - & - \\
\hline $04-26-00$ & 1635 & - & 16.0 & 384 & 8.5 & $.7 \mathrm{E}$ & - & - \\
\hline $06-28-00$ & 1320 & - & 17.0 & 335 & 8.3 & $.7 \mathrm{E}$ & - & - \\
\hline $07-26-00$ & 1330 & - & 19.0 & 295 & 8.4 & $.4 \mathrm{E}$ & - & - \\
\hline 08-30-00 & 1645 & - & 19.5 & 295 & 8.5 & 1.1 & - & - \\
\hline \multicolumn{9}{|c|}{ NW 1 Seep to Burns Bench Canal, north of U.S. 40 (NW1) } \\
\hline $03-15-95$ & 0910 & .04 & 7.0 & 10,000 & - & 230 & - & - \\
\hline \multicolumn{9}{|c|}{ NW 2 Seep to Burns Bench Canal, north of U.S. 40 (NW2) } \\
\hline $03-15-95$ & 0930 & .16 & 7.0 & 9,400 & - & 290 & - & - \\
\hline \multicolumn{9}{|c|}{ NW 3 Seep to Burns Bench Canal, north of U.S. 40 (NW3) } \\
\hline $03-15-95$ & 0945 & .01 & 8.5 & 7,900 & - & 220 & - & - \\
\hline \multicolumn{9}{|c|}{ Burns Bench Canal at U.S. 40 (BBC 40) } \\
\hline $03-15-95$ & 1330 & .17 & 11.0 & 5,600 & - & 110 & - & - \\
\hline \multicolumn{9}{|c|}{ SW 1 Seep to Burns Bench Canal, south of U.S. 40 (SW1) } \\
\hline $03-15-95$ & 1020 & .05 & 9.0 & 9,500 & - & 230 & - & - \\
\hline
\end{tabular}


Table 6. Discharge, physical properties, selenium concentration, and depth of water at selected sample locations in Brush Creek drainage near Jensen, Utah, water years 1991-2000-Continued

\begin{tabular}{|c|c|c|c|c|c|c|c|c|}
\hline Date & Time & $\begin{array}{c}\text { Discharge, } \\
\text { instantaneous } \\
\left(\mathrm{ft}^{3} / \mathrm{s}\right)\end{array}$ & $\begin{array}{c}\text { Temper- } \\
\text { ature } \\
\left({ }^{\circ} \mathrm{C}\right)\end{array}$ & $\begin{array}{c}\text { Specific } \\
\text { conductance } \\
(\mu \mathrm{S} / \mathrm{cm})\end{array}$ & $\begin{array}{l}\mathrm{pH}, \text { water, } \\
\text { whole, field } \\
\text { (standard } \\
\text { units) }\end{array}$ & $\begin{array}{c}\text { Selenium, } \\
\text { dissolved } \\
(\mu \mathrm{g} / \mathrm{L} \\
\text { as Se) }\end{array}$ & $\begin{array}{c}\text { Selenium, } \\
\text { total } \\
(\mu \mathrm{g} / \mathrm{L} \\
\text { as Se) }\end{array}$ & $\begin{array}{c}\text { Depth at } \\
\text { sample } \\
\text { location, } \\
\text { total } \\
\text { (feet) }\end{array}$ \\
\hline \multicolumn{9}{|c|}{ Burns Bench Canal at diversion dam south of U.S. 40 (BBC Dam) } \\
\hline $03-15-95$ & 1130 & .33 & 9.0 & 4,850 & - & 110 & - & - \\
\hline 08-14-95 & 1600 & 5.4 & 19.5 & 580 & - & 5.0 & 5.0 & - \\
\hline $10-24-96$ & 1400 & - & 5.0 & 1,120 & 8.1 & 6.9 & - & - \\
\hline \multicolumn{9}{|c|}{ Pipeline from Burns Bench Canal to Stewart Lake (BBC Pipe) } \\
\hline $04-16-96$ & 1400 & $.03 \mathrm{E}$ & 9.5 & 1,590 & 8.3 & 25 & - & - \\
\hline 06-13-96 & 1100 & 1.0 & 16.0 & 620 & 8.0 & 4.0 & - & - \\
\hline 07-16-96 & 1555 & - & 19.0 & 830 & 8.8 & 8.0 & - & - \\
\hline 08-20-96 & 0750 & - & 15.5 & 570 & 8.0 & 5.0 & - & - \\
\hline 05-14-97 & 0930 & .50 & 9.0 & 660 & 8.2 & 4.0 & - & - \\
\hline 06-10-97 & 0800 & $2.0 \mathrm{E}$ & 14.0 & 600 & 8.2 & 4.2 & - & - \\
\hline 06-10-97 & 1500 & - & - & 585 & 8.1 & - & 4.3 & - \\
\hline 07-17-97 & 0930 & $1.0 \mathrm{E}$ & 16.0 & 585 & 8.2 & 15 & - & - \\
\hline 08-19-97 & 1100 & - & 18.0 & 660 & 8.3 & 4.8 & - & - \\
\hline 09-16-97 & 1130 & - & 15.0 & 660 & 8.3 & 6.1 & - & - \\
\hline 01-26-99 & 1300 & - & - & - & - & - & 22 & - \\
\hline \multicolumn{9}{|c|}{ Brush Creek near Jensen, Utah (BC) } \\
\hline $08-12-93$ & 0940 & - & - & 390 & - & - & - & - \\
\hline $10-25-94$ & 1345 & .29 & 12.0 & 2,200 & 7.9 & 23 & - & - \\
\hline 09-16-99 & 1215 & 1.0 & 16.5 & 1,890 & 7.9 & - & - & - \\
\hline $03-08-00$ & 1300 & - & - & 600 & - & 2.1 & - & - \\
\hline
\end{tabular}


Table 7. Discharge, physical properties, and selected chemical analyses of surface-water inflow to Stewart Lake near Jensen, Utah, water years $1991-2000$

[Data from U.S. Geological Survey; $\mathrm{ft}^{3} / \mathrm{s}$, cubic feet per second; ${ }^{\circ} \mathrm{C}$, degrees Celsius; $\mu \mathrm{S} / \mathrm{cm}$, microsiemens per centimeter at $25{ }^{\circ} \mathrm{C} ; \mathrm{mg} / \mathrm{L}$, milligrams per liter; $\mu \mathrm{g} / \mathrm{L}$, micrograms per liter; —, no data; <, less than; E, estimated]

\begin{tabular}{|c|c|c|c|c|c|c|c|c|c|c|c|c|}
\hline Date & Time & $\begin{array}{c}\text { Discharge, } \\
\text { instan- } \\
\text { taneous } \\
\left(\mathrm{ft}^{3} / \mathrm{s}\right)\end{array}$ & $\begin{array}{l}\text { Temper- } \\
\text { ature } \\
\left({ }^{\circ} \mathrm{C}\right)\end{array}$ & $\begin{array}{c}\mathrm{pH}, \\
\text { water, } \\
\text { whole, } \\
\text { field } \\
\text { (standard } \\
\text { units) }\end{array}$ & $\begin{array}{c}\text { Specific } \\
\text { conduc- } \\
\text { tance } \\
(\mu \mathrm{S} / \mathrm{cm})\end{array}$ & $\begin{array}{l}\text { Solids, } \\
\text { residue at } \\
180^{\circ} \mathrm{C}, \\
\text { dissolved } \\
\text { (mg/L) }\end{array}$ & $\begin{array}{c}\text { Boron, } \\
\text { dis- } \\
\text { solved } \\
(\mu \mathrm{g} / L \text { as } B)\end{array}$ & $\begin{array}{c}\text { Selenium, } \\
\text { dis- } \\
\text { solved } \\
(\mu \mathrm{g} / \mathrm{L} \text { as } \\
\mathrm{Se})\end{array}$ & $\begin{array}{l}\text { Selenium, } \\
\text { total } \\
(\mu \mathrm{g} / \mathrm{L} \text { as } \\
\mathrm{Se})\end{array}$ & $\begin{array}{l}\text { Uranium, } \\
\text { natural, } \\
\text { dissolved } \\
(\mu g / L \\
\text { as U) }\end{array}$ & $\begin{array}{l}\text { Uranium, } \\
\text { natural, } \\
2 \text { sigma } \\
\text { water, } \\
\text { dissolved } \\
(\mu \mathrm{g} / \mathrm{L})\end{array}$ & $\begin{array}{c}\text { Zinc, } \\
\text { dis- } \\
\text { solved } \\
(\mu g / L \text { as } \\
\text { Zn) }\end{array}$ \\
\hline \multicolumn{13}{|c|}{ J1 drain, Stewart Lake inflow (J1) } \\
\hline $06-20-91$ & 0920 & - & 11.0 & 7.1 & 1,950 & - & - & 26 & - & - & - & - \\
\hline 06-04-92 & 1455 & - & 10.5 & 7.3 & 2,500 & - & - & 34 & - & - & - & - \\
\hline 08-17-92 & 1845 & - & 13.5 & 7.2 & 2,400 & - & - & 35 & - & - & - & - \\
\hline $03-25-93$ & 1340 & .55 & - & 7.6 & 2,660 & 2,190 & 160 & 35 & - & 42 & 6.3 & $<10$ \\
\hline $04-21-93$ & 1040 & .18 & 8.5 & 7.4 & 2,500 & 2,140 & 600 & 38 & - & 36 & 5.4 & $<10$ \\
\hline $05-25-93$ & 1100 & 1.5 & 9.5 & 7.2 & 2,430 & 2,030 & 590 & 87 & - & 35 & 5.3 & 10 \\
\hline $03-17-94$ & 1110 & .16 & 8.0 & 7.4 & 1,310 & 970 & 180 & 14 & - & 13 & 1.9 & $<10$ \\
\hline 04-13-94 & 1020 & .04 & 9.0 & 7.2 & 1,530 & 1,190 & 190 & 10 & - & 15 & 2.2 & $<10$ \\
\hline 05-18-94 & 0935 & .67 & 9.5 & 7.2 & 2,640 & 2,300 & 690 & 46 & - & 45 & 6.8 & $<10$ \\
\hline 06-16-94 & 0925 & 2.0 & 11.0 & 7.2 & 2,290 & 1,990 & 580 & 33 & - & 37 & 5.5 & $<10$ \\
\hline 07-14-94 & 0820 & 2.0 & 12.0 & 7.2 & 2,230 & 2,130 & 680 & 32 & - & 35 & 5.2 & $<10$ \\
\hline $08-17-94$ & 0710 & 1.7 & 13.5 & 7.2 & 2,440 & 1,970 & 660 & 37 & - & 37 & 5.5 & $<10$ \\
\hline $09-21-94$ & 0800 & .88 & 14.0 & 7.2 & 2,380 & 2,050 & 650 & 30 & - & 36 & 5.5 & $<10$ \\
\hline $10-19-94$ & 0910 & .82 & 13.5 & 7.2 & 2,420 & 2,080 & 690 & 31 & - & 41 & 2.5 & $<10$ \\
\hline $02-15-95$ & 1405 & .15 & 9.0 & 7.8 & 2,230 & 1,870 & 520 & 22 & - & - & - & $<10$ \\
\hline 03-21-95 & 0935 & .11 & 9.0 & 7.5 & 2,050 & 1,720 & 460 & 20 & - & 32 & 2.0 & 40 \\
\hline $03-26-96$ & 0945 & .05 & 7.5 & 8.0 & 1,410 & 1,010 & 250 & 16 & - & - & - & $<10$ \\
\hline 04-16-96 & 0825 & .04 & 9.0 & 8.0 & 1,450 & 984 & 30 & 15 & - & - & - & $<10$ \\
\hline 05-16-96 & 0820 & 1.6 & 9.5 & 7.2 & 2,660 & 2,240 & - & 36 & - & - & - & - \\
\hline 06-13-96 & 0910 & .49 & 10.0 & 7.1 & 2,310 & 1,960 & - & 30 & - & - & - & - \\
\hline $07-16-96$ & 1040 & 1.0 & 12.0 & 7.2 & 2,340 & 2,020 & - & 26 & - & - & - & - \\
\hline 08-20-96 & 0810 & 2.4 & 13.5 & 7.2 & 2,580 & 2,020 & - & 31 & - & - & - & - \\
\hline 09-19-96 & 1045 & 1.2 & 13.5 & 7.3 & 2,450 & 2,110 & - & 34 & - & - & - & - \\
\hline $10-24-96$ & 0845 & .55 & 13.5 & 7.2 & 2,440 & 2,070 & - & 37 & - & - & - & - \\
\hline 03-20-97 & 0905 & .18 & - & 7.4 & 2,240 & 1,940 & - & 17 & - & - & - & - \\
\hline 04-16-97 & 0800 & .04 & - & 7.8 & 1,960 & 1,550 & - & 22 & - & - & - & - \\
\hline $05-14-97$ & 0845 & .40 & 8.5 & 7.4 & 2,820 & 2,470 & - & 36 & - & - & - & - \\
\hline 06-10-97 & 0830 & 1.2 & 10.0 & 7.4 & 2,430 & 2,020 & - & 34 & - & - & - & - \\
\hline $07-17-97$ & 0945 & 3.1 & 12.0 & 7.2 & 2,400 & 2,110 & - & 33 & - & - & - & - \\
\hline 08-19-97 & 0705 & 2.0 & 12.5 & 7.2 & 2,660 & 2,330 & - & 26 & - & - & - & - \\
\hline $09-16-97$ & 0755 & .44 & 13.0 & 7.2 & 2,370 & - & - & 30 & - & - & - & - \\
\hline
\end{tabular}


Table 7. Discharge, physical properties, and selected chemical analyses of surface-water inflow to Stewart Lake near Jensen, Utah, water years 1991 2000-Continued

\begin{tabular}{|c|c|c|c|c|c|c|c|c|c|c|c|c|}
\hline Date & Time & $\begin{array}{c}\text { Discharge, } \\
\text { instan- } \\
\text { taneous } \\
\left(\mathrm{ft}^{3} / \mathrm{s}\right)\end{array}$ & $\begin{array}{l}\text { Temper- } \\
\text { ature } \\
\left({ }^{\circ} \mathrm{C}\right)\end{array}$ & $\begin{array}{c}\mathrm{pH}, \\
\text { water, } \\
\text { whole, } \\
\text { field } \\
\text { (standard } \\
\text { units) }\end{array}$ & $\begin{array}{c}\text { Specific } \\
\text { conduc- } \\
\text { tance } \\
(\mu \mathrm{S} / \mathrm{cm})\end{array}$ & $\begin{array}{l}\text { Solids, } \\
\text { residue at } \\
180^{\circ} \mathrm{C}, \\
\text { dissolved } \\
\text { (mg/L) }\end{array}$ & $\begin{array}{c}\text { Boron, } \\
\text { dis- } \\
\text { solved } \\
(\mu \mathrm{g} / L \text { as } B)\end{array}$ & $\begin{array}{c}\text { Selenium, } \\
\text { dis- } \\
\text { solved } \\
\text { ( } \mu \mathrm{g} / \mathrm{L} \text { as } \\
\mathrm{Se})\end{array}$ & $\begin{array}{c}\text { Selenium, } \\
\text { total } \\
(\mu \mathrm{g} / \mathrm{L} \text { as } \\
\text { Se })\end{array}$ & $\begin{array}{c}\text { Uranium, } \\
\text { natural, } \\
\text { dissolved } \\
(\mu \mathrm{g} / \mathrm{L} \\
\text { as U) }\end{array}$ & $\begin{array}{c}\text { Uranium, } \\
\text { natural, } \\
2 \text { sigma } \\
\text { water, } \\
\text { dissolved } \\
(\mu \mathrm{g} / \mathrm{L})\end{array}$ & $\begin{array}{c}\text { Zinc, } \\
\text { dis- } \\
\text { solved } \\
(\mu g / L \text { as } \\
\mathrm{Zn})\end{array}$ \\
\hline \multicolumn{13}{|c|}{ J1 drain, Stewart Lake inflow (J1)_Continued } \\
\hline $10-07-97$ & 1130 & 1.3 & 13.5 & 7.5 & 2,500 & - & - & 30 & - & - & - & - \\
\hline 03-25-98 & 0810 & .05 & 7.5 & 7.6 & 1,740 & - & - & 18 & - & - & - & - \\
\hline $05-20-98$ & 0755 & .63 & 10.0 & 7.4 & 2,560 & - & - & 23 & - & - & - & - \\
\hline $06-16-98$ & 0945 & 2.5 & 11.0 & 7.3 & 2,000 & - & - & 26 & - & - & - & - \\
\hline \multicolumn{13}{|c|}{ J1A drain, Stewart Lake inflow (J1A) } \\
\hline$\overline{06-20-91}$ & 0925 & - & 11.0 & 7.2 & 1,220 & - & - & 13 & - & - & - & - \\
\hline $10-23-91$ & 0850 & .38 & 14.0 & - & 1,180 & - & - & 7.0 & - & - & - & - \\
\hline $04-21-93$ & 1045 & .10 & 8.5 & 7.2 & 1,410 & 1,060 & 190 & 11 & - & 12 & 1.7 & $<10$ \\
\hline $05-25-93$ & 1105 & .62 & 10.0 & 7.2 & 1,350 & 934 & 170 & 12 & - & 12 & 1.8 & $<10$ \\
\hline $06-24-93$ & 0835 & .28 & 13.0 & 7.1 & 1,250 & 928 & 180 & 11 & - & 10 & 1.5 & $<10$ \\
\hline $07-28-93$ & 0935 & .46 & 13.0 & 7.2 & 1,180 & 892 & 180 & 10 & - & 11 & 1.7 & $<10$ \\
\hline $08-25-93$ & 0830 & .86 & 13.0 & 7.1 & 1,350 & 910 & 20 & 10 & - & 12 & 1.7 & $<10$ \\
\hline $10-13-93$ & 0845 & .24 & 13.5 & 7.2 & 1,170 & 828 & 190 & 7.0 & - & 9.4 & 1.4 & $<10$ \\
\hline $03-17-94$ & 1100 & .05 & 8.0 & 7.2 & 1,520 & 1,130 & 180 & 10 & - & 14 & 2.1 & $<10$ \\
\hline 04-13-94 & 1015 & .01 & 8.5 & 7.7 & 1,320 & 986 & 190 & 16 & - & 12 & 1.8 & $<10$ \\
\hline $05-18-94$ & 1015 & .32 & 9.5 & 7.2 & 1,220 & 892 & 160 & 8.0 & - & 11 & 1.7 & $<10$ \\
\hline $06-16-94$ & 0925 & .51 & 11.0 & 7.2 & 1,190 & 834 & 150 & 7.0 & - & 10 & 1.6 & - \\
\hline $05-18-95$ & 0910 & .41 & 9.5 & 7.1 & 1,440 & 1,030 & 170 & 9.0 & - & 14 & .9 & $<10$ \\
\hline $06-22-95$ & 0905 & .40 & 11.0 & 7.1 & 1,460 & 1,080 & 200 & 12 & - & 14 & .9 & $<10$ \\
\hline $07-18-95$ & 0855 & .67 & 12.0 & 7.0 & 1,320 & 964 & 190 & 9.0 & - & 12 & .3 & $<10$ \\
\hline $08-17-95$ & 0810 & .97 & 15.0 & 7.2 & 1,330 & 968 & 200 & 10 & - & 13 & .8 & $<10$ \\
\hline 09-23-95 & 0730 & .39 & 15.0 & 7.2 & 1,250 & 914 & 190 & 8.0 & - & 12 & .7 & $<10$ \\
\hline $10-25-95$ & 0900 & .31 & 13.5 & 7.2 & 1,310 & 966 & 190 & 9.0 & - & 12 & .8 & $<10$ \\
\hline $03-26-96$ & 0950 & .06 & 7.5 & 7.4 & 1,690 & 1,290 & 220 & 10 & - & - & - & $<10$ \\
\hline 04-16-96 & 0830 & .05 & 8.0 & 7.4 & 1,730 & 1,250 & 240 & 11 & - & - & - & $<10$ \\
\hline $05-16-96$ & 0840 & .70 & 9.5 & 7.1 & 1,530 & 1,120 & - & 12 & - & - & - & - \\
\hline $06-13-96$ & 0900 & .44 & 10.5 & 7.0 & 1,460 & 864 & - & 8.0 & - & - & - & - \\
\hline $07-16-96$ & 1050 & 1.0 & 13.0 & 7.2 & 1,310 & 954 & - & 10 & - & - & - & - \\
\hline $08-20-96$ & 0900 & .44 & 14.5 & 7.2 & 1,500 & 1,030 & - & 10 & - & - & - & - \\
\hline 09-19-96 & 1115 & .69 & 15.0 & 7.3 & 1,270 & 930 & - & 8.0 & - & - & - & - \\
\hline $10-24-96$ & 0850 & .36 & 14.5 & 7.2 & 1,260 & - & - & 5.8 & - & - & - & - \\
\hline 03-20-97 & 0905 & .06 & 6.0 & 7.2 & 1,600 & - & - & 11 & - & - & - & - \\
\hline $4-16-97$ & 0925 & .01 & 6.5 & 7.3 & 1,670 & - & - & 9.8 & - & - & - & - \\
\hline
\end{tabular}


Table 7. Discharge, physical properties, and selected chemical analyses of surface-water inflow to Stewart Lake near Jensen, Utah, water years 19912000-Continued

\begin{tabular}{|c|c|c|c|c|c|c|c|c|c|c|c|c|}
\hline Date & Time & $\begin{array}{c}\text { Discharge, } \\
\text { instan- } \\
\text { taneous } \\
\left(\mathrm{ft}^{3} / \mathrm{s}\right)\end{array}$ & $\begin{array}{l}\text { Temper- } \\
\text { ature } \\
\left({ }^{\circ} \mathrm{C}\right)\end{array}$ & $\begin{array}{c}\mathrm{pH}, \\
\text { water, } \\
\text { whole, } \\
\text { field } \\
\text { (standard } \\
\text { units) }\end{array}$ & $\begin{array}{c}\text { Specific } \\
\text { conduc- } \\
\text { tance } \\
(\mu \mathrm{S} / \mathrm{cm})\end{array}$ & $\begin{array}{l}\text { Solids, } \\
\text { residue at } \\
180^{\circ} \mathrm{C}, \\
\text { dissolved } \\
\text { (mg/L) }\end{array}$ & $\begin{array}{c}\text { Boron, } \\
\text { dis- } \\
\text { solved } \\
(\mu \mathrm{g} / L \text { as } B)\end{array}$ & $\begin{array}{c}\text { Selenium, } \\
\text { dis- } \\
\text { solved } \\
(\mu g / L \text { as } \\
\text { Se) }\end{array}$ & $\begin{array}{c}\text { Selenium, } \\
\text { total } \\
(\mu \mathrm{g} / \mathrm{L} \text { as } \\
\mathrm{Se})\end{array}$ & $\begin{array}{c}\text { Uranium, } \\
\text { natural, } \\
\text { dissolved } \\
(\mu \mathrm{g} / \mathrm{L} \\
\text { as U) }\end{array}$ & $\begin{array}{l}\text { Uranium, } \\
\text { natural, } \\
2 \text { sigma } \\
\text { water, } \\
\text { dissolved } \\
(\mu \mathrm{g} / \mathrm{L})\end{array}$ & 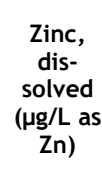 \\
\hline \multicolumn{13}{|c|}{ J1A drain, Stewart Lake inflow (J1A)—Continued } \\
\hline $5-14-97$ & 0905 & .10 & 9.0 & 7.3 & 1,680 & - & - & 11 & - & - & - & - \\
\hline $8-19-97$ & 0710 & .40 & 13.0 & 7.1 & 1,720 & - & - & 12 & - & - & - & - \\
\hline $09-16-97$ & 0800 & .42 & 14.0 & 7.2 & 1,360 & - & - & 10 & - & - & - & - \\
\hline $10-07-97$ & 1145 & .36 & 14.0 & 7.2 & 1,510 & - & - & 11 & - & - & - & - \\
\hline $03-25-98$ & 0815 & .04 & 8.0 & 7.4 & 1,750 & - & - & 16 & - & - & - & - \\
\hline $04-29-98$ & 0740 & .06 & 9.0 & 7.3 & 1,790 & - & - & 14 & - & - & - & - \\
\hline $08-21-91$ & 1430 & - & 14.0 & - & - & 1,670 & - & 27 & - & - & - & - \\
\hline $08-27-91$ & 1640 & .06 & 19.0 & 7.4 & 1,220 & - & - & 6.0 & - & - & - & - \\
\hline $04-07-92$ & 1610 & .10 & 9.0 & 7.2 & 2,220 & - & - & 12 & - & - & - & - \\
\hline 06-03-92 & 1730 & 1.1 & 11.0 & 7.0 & 2,280 & - & - & 28 & - & - & - & - \\
\hline $08-17-92$ & 1800 & - & 14.5 & 7.2 & 1,950 & - & - & 23 & - & - & - & - \\
\hline $03-25-93$ & 1415 & .44 & 8.5 & 7.5 & 2,270 & 1,800 & 560 & 25 & - & 32 & 4.8 & $<10$ \\
\hline $04-21-93$ & 1240 & .17 & 8.5 & 7.1 & 2,180 & 1,880 & 540 & 32 & - & 31 & 4.7 & $<10$ \\
\hline $05-26-93$ & 1135 & .94 & 10.0 & 7.1 & 2,120 & 1,680 & 500 & 29 & - & 31 & 4.7 & $<10$ \\
\hline $06-24-93$ & 0955 & 1.2 & 10.5 & 7.0 & 2,050 & 1,610 & 470 & 19 & - & 26 & 3.9 & $<10$ \\
\hline $07-28-93$ & 1040 & 1.2 & 12.5 & 7.1 & 2,010 & 1,660 & 490 & 20 & - & 29 & 4.3 & $<10$ \\
\hline $08-17-94$ & 0840 & 1.3 & 14.0 & 7.1 & 1,920 & 1,470 & 470 & 19 & - & 25 & 3.8 & $<10$ \\
\hline $09-21-94$ & 1030 & .22 & 14.0 & 7.6 & 2,380 & 1,500 & 460 & 18 & - & 26 & 3.8 & $<10$ \\
\hline $10-19-94$ & 1015 & .54 & 13.5 & 7.2 & 1,950 & 1,570 & 520 & 17 & - & 31 & 1.9 & $<10$ \\
\hline $02-15-95$ & 1520 & .07 & 8.5 & 7.6 & 2,080 & 1,730 & 480 & 21 & - & - & - & $<10$ \\
\hline $03-21-95$ & 1010 & .03 & 9.0 & 7.4 & 2,070 & 1,750 & 480 & 21 & - & 33 & 2.1 & $<10$ \\
\hline 04-19-95 & 1025 & .02 & 8.5 & 7.7 & 2,130 & 1,730 & 490 & 20 & - & 31 & 1.9 & $<10$ \\
\hline 05-18-95 & 0930 & .20 & 10.0 & 7.2 & 2,230 & 1,860 & 470 & 33 & - & 36 & 2.3 & $<10$ \\
\hline $06-22-95$ & 1050 & .40 & 11.5 & 7.2 & 2,110 & 1,760 & 510 & 22 & - & 33 & 2.1 & $<10$ \\
\hline $07-18-95$ & 1015 & .57 & 12.0 & 7.0 & 2,000 & 1,640 & 500 & 20 & - & 30 & 1.8 & $<10$ \\
\hline $08-17-95$ & 0955 & - & 14.0 & 7.1 & 1,800 & 1,430 & 430 & 16 & - & 27 & 1.7 & $<10$ \\
\hline 09-23-95 & 0740 & .62 & 19.5 & 7.2 & 1,870 & 1,530 & 460 & 19 & - & 29 & 1.8 & $<10$ \\
\hline $10-25-95$ & 0910 & .46 & 13.0 & 7.2 & 2,010 & 1,670 & 540 & 18 & - & 30 & 1.9 & $<10$ \\
\hline $03-26-96$ & 1130 & .05 & 7.5 & 7.6 & 2,050 & 1,650 & 430 & 23 & - & - & - & $<10$ \\
\hline $04-16-96$ & 1410 & $5.0 \mathrm{E}$ & 9.5 & 7.7 & 2,070 & 1,590 & 470 & 17 & - & - & - & $<10$ \\
\hline $05-16-96$ & 1140 & .76 & 9.0 & 7.1 & 2,110 & 1,690 & - & 28 & - & - & - & - \\
\hline 06-13-96 & 1120 & 1.6 & 10.5 & 6.9 & 1,940 & 1,560 & - & 23 & - & - & - & - \\
\hline
\end{tabular}


Table 7. Discharge, physical properties, and selected chemical analyses of surface-water inflow to Stewart Lake near Jensen, Utah, water years 1991 2000-Continued

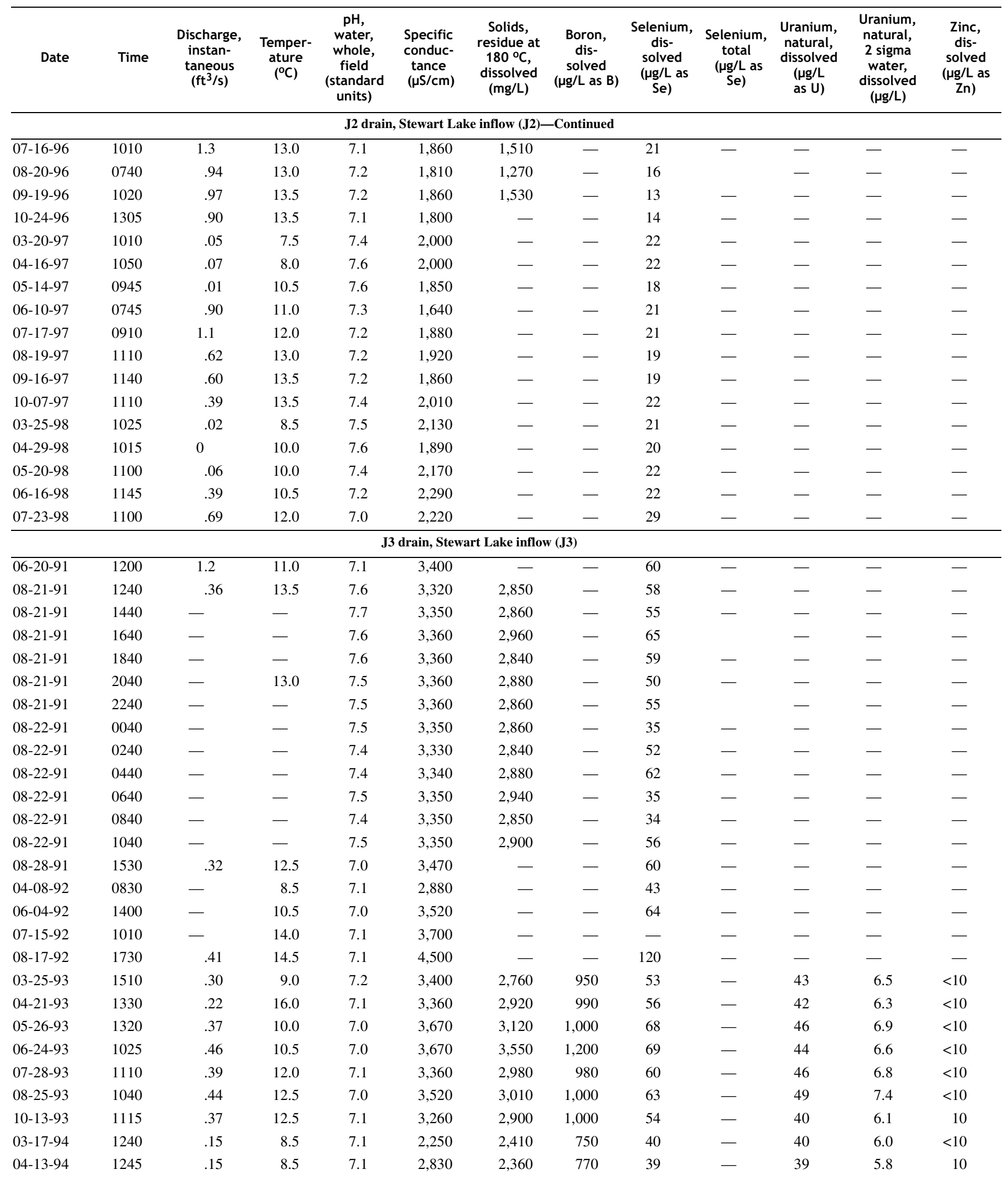


Table 7. Discharge, physical properties, and selected chemical analyses of surface-water inflow to Stewart Lake near Jensen, Utah, water years 19912000-Continued

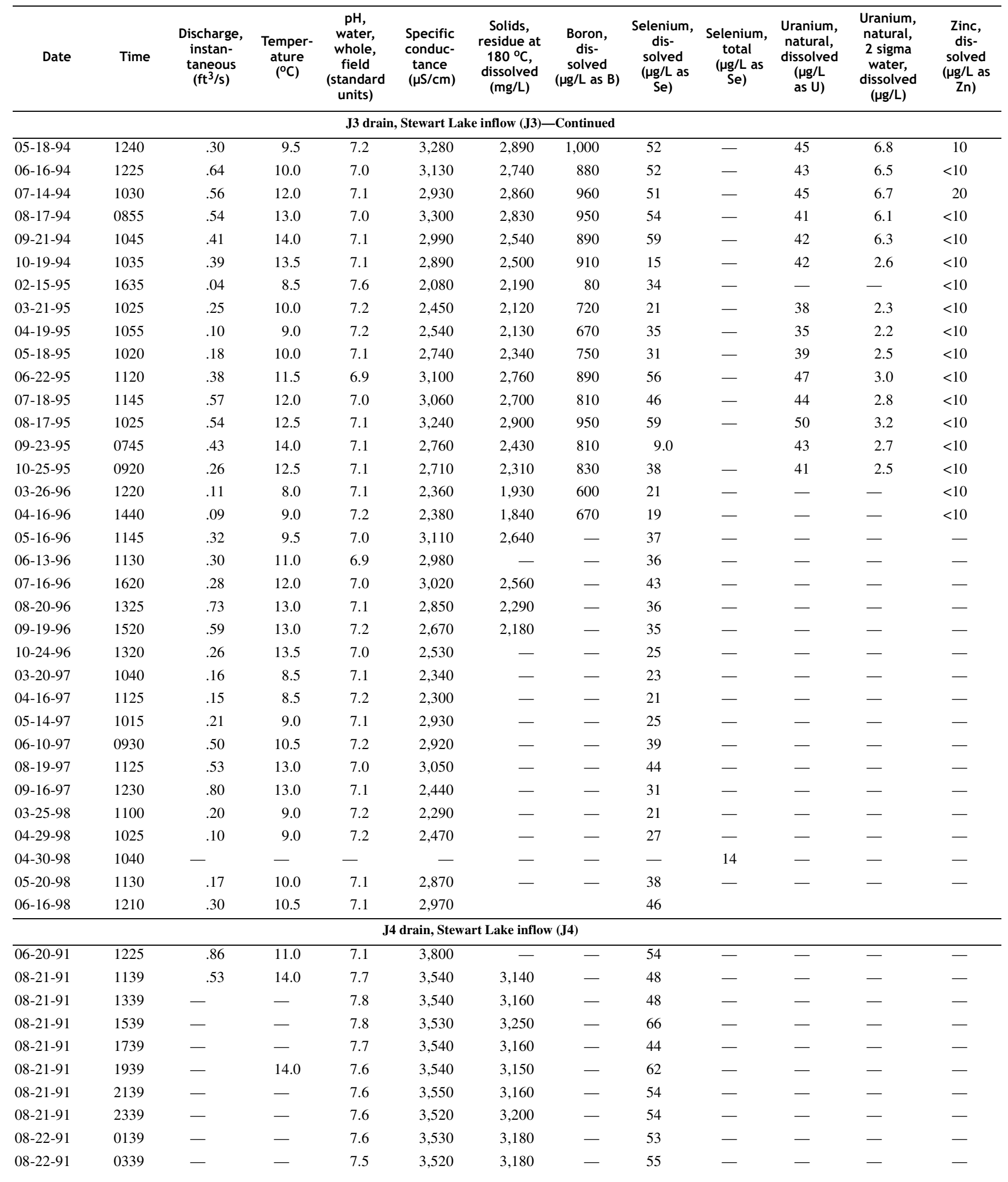


Table 7. Discharge, physical properties, and selected chemical analyses of surface-water inflow to Stewart Lake near Jensen, Utah, water years 19912000-Continued

\begin{tabular}{|c|c|c|c|c|c|c|c|c|c|c|c|c|}
\hline Date & Time & $\begin{array}{c}\text { Discharge, } \\
\text { instan- } \\
\text { taneous } \\
\left(\mathrm{ft}^{3} / \mathrm{s}\right)\end{array}$ & $\begin{array}{l}\text { Temper- } \\
\text { ature } \\
\left({ }^{\circ} \mathrm{C}\right)\end{array}$ & $\begin{array}{c}\mathrm{pH}, \\
\text { water, } \\
\text { whole, } \\
\text { field } \\
\text { (standard } \\
\text { units) }\end{array}$ & $\begin{array}{c}\text { Specific } \\
\text { conduc- } \\
\text { tance } \\
(\mu \mathrm{S} / \mathrm{cm})\end{array}$ & $\begin{array}{l}\text { Solids, } \\
\text { residue at } \\
180^{\circ} \mathrm{C}, \\
\text { dissolved } \\
\text { (mg/L) }\end{array}$ & $\begin{array}{c}\text { Boron, } \\
\text { dis- } \\
\text { solved } \\
(\mu \mathrm{g} / \mathrm{L} \text { as } B)\end{array}$ & $\begin{array}{c}\text { Selenium, } \\
\text { dis- } \\
\text { solved } \\
(\mu \mathrm{g} / \mathrm{L} \text { as } \\
\text { Se) }\end{array}$ & $\begin{array}{c}\text { Selenium, } \\
\text { total } \\
(\mu \mathrm{g} / \mathrm{L} \text { as } \\
\text { Se })\end{array}$ & $\begin{array}{l}\text { Uranium, } \\
\text { natural, } \\
\text { dissolved } \\
(\mu \mathrm{g} / \mathrm{L} \\
\text { as U) }\end{array}$ & $\begin{array}{l}\text { Uranium, } \\
\text { natural, } \\
2 \text { sigma } \\
\text { water, } \\
\text { dissolved } \\
(\mu \mathrm{g} / L)\end{array}$ & 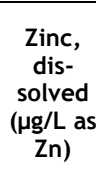 \\
\hline \multicolumn{13}{|c|}{ J4 drain, Stewart Lake inflow (J4)—Continued } \\
\hline $08-22-91$ & 0539 & - & - & 7.5 & 3,510 & 3,180 & - & 49 & - & - & - & - \\
\hline $08-22-91$ & 0739 & - & - & 7.5 & 3,500 & 3,180 & - & 54 & - & - & - & - \\
\hline 08-28-91 & 1600 & .61 & 29.0 & 7.0 & 3,570 & - & - & 74 & - & - & - & - \\
\hline 04-08-92 & 0850 & - & 7.5 & 7.0 & 4,250 & - & - & 70 & - & - & - & - \\
\hline 06-04-92 & 1415 & - & 11.0 & 7.2 & 2,590 & - & - & 26 & - & - & - & - \\
\hline $07-15-92$ & 0940 & .81 & 14.0 & 7.2 & 2,850 & - & - & - & - & - & - & - \\
\hline 08-17-92 & 1700 & 1.1 & 14.5 & 7.1 & 3,500 & - & - & 52 & - & - & - & - \\
\hline 07-28-93 & 1140 & .67 & 12.0 & 7.1 & 3,290 & 2,920 & 930 & 52 & - & 41 & 6.1 & $<10$ \\
\hline 08-25-93 & 1100 & .05 & 13.0 & 7.1 & 3,530 & 3,030 & 1,000 & 57 & - & 42 & 6.3 & $<10$ \\
\hline $10-13-93$ & 1145 & .34 & 13.0 & 7.2 & 3,390 & 3,030 & 1,000 & 48 & - & 37 & 5.6 & 10 \\
\hline $03-17-94$ & 1400 & .07 & 7.0 & 7.2 & 4,450 & 4,020 & 1,100 & 56 & - & 50 & 7.5 & $<10$ \\
\hline 04-13-94 & 1300 & .10 & 8.0 & 7.0 & 4,250 & 3,980 & 1,100 & 62 & - & 49 & 7.4 & $<10$ \\
\hline 05-18-94 & 1300 & .76 & 11.0 & 7.4 & 2,330 & 1,990 & 590 & 26 & - & 27 & 4.0 & $<10$ \\
\hline 06-16-94 & 1245 & .76 & 10.5 & 7.1 & 3,480 & 3,080 & 870 & 52 & - & 42 & 6.2 & $<10$ \\
\hline 07-14-94 & 0945 & .81 & 12.0 & 7.1 & 3,300 & 2,900 & 960 & 41 & - & 38 & 5.7 & $<10$ \\
\hline 08-17-94 & 0910 & 1.3 & 13.0 & 7.1 & 2,950 & 2,470 & 760 & 43 & - & 35 & 5.2 & $<10$ \\
\hline 09-21-94 & 1100 & .31 & 14.0 & 7.1 & 3,670 & 3,240 & 1,000 & 51 & - & 40 & 6.1 & $<10$ \\
\hline 08-17-95 & 1050 & 1.3 & 14.5 & 7.1 & 2,380 & 2,010 & 590 & 31 & - & 30 & 1.9 & $<10$ \\
\hline 09-23-95 & 0800 & .38 & 14.0 & 7.1 & 3,370 & 3,040 & 950 & 48 & - & 43 & .9 & $<10$ \\
\hline $10-25-95$ & 0950 & .15 & 13.0 & 7.2 & 4,220 & 3,870 & 1,200 & 37 & - & 50 & 3.1 & $<10$ \\
\hline $03-26-96$ & 1210 & .07 & 7.0 & 7.2 & 4,520 & 4,130 & 1,100 & 57 & - & - & - & $<10$ \\
\hline 04-16-96 & 1500 & .07 & 9.0 & 7.2 & 4,480 & 3,920 & 1,300 & 56 & - & - & - & $<10$ \\
\hline 05-16-96 & 1215 & .58 & 10.0 & 7.1 & 3,750 & 3,320 & - & 41 & - & - & - & - \\
\hline $06-13-96$ & 1230 & .96 & 10.5 & 6.9 & 3,480 & 3,120 & - & 49 & - & - & - & - \\
\hline 07-16-96 & 1645 & .96 & 12.0 & 7.0 & 3,670 & 3,210 & - & 60 & - & - & - & - \\
\hline 08-20-96 & 1350 & .86 & 13.5 & 7.1 & 3,040 & - & - & 37 & - & - & - & - \\
\hline 09-19-96 & 1500 & .42 & 14.0 & 7.2 & 3,440 & 2,800 & - & 42 & - & - & - & - \\
\hline $10-24-96$ & 1345 & .21 & 13.5 & 7.0 & 3,620 & - & - & 40 & - & - & - & - \\
\hline 03-20-97 & 1105 & .06 & 7.0 & 7.2 & 4,420 & - & - & 51 & - & - & - & - \\
\hline $04-17-97$ & 1110 & .05 & 7.5 & 7.2 & 4,630 & - & - & 70 & - & - & - & - \\
\hline 05-14-97 & 1045 & .27 & 9.5 & 7.2 & 4,370 & - & - & 68 & - & - & - & - \\
\hline 06-10-97 & 0955 & .14 & 10.5 & 7.2 & 3,630 & - & - & 50 & - & - & - & - \\
\hline 08-10-97 & 1150 & .96 & 13.0 & 7.1 & 3,500 & - & - & 45 & - & - & - & - \\
\hline
\end{tabular}


Table 7. Discharge, physical properties, and selected chemical analyses of surface-water inflow to Stewart Lake near Jensen, Utah, water years 19912000-Continued

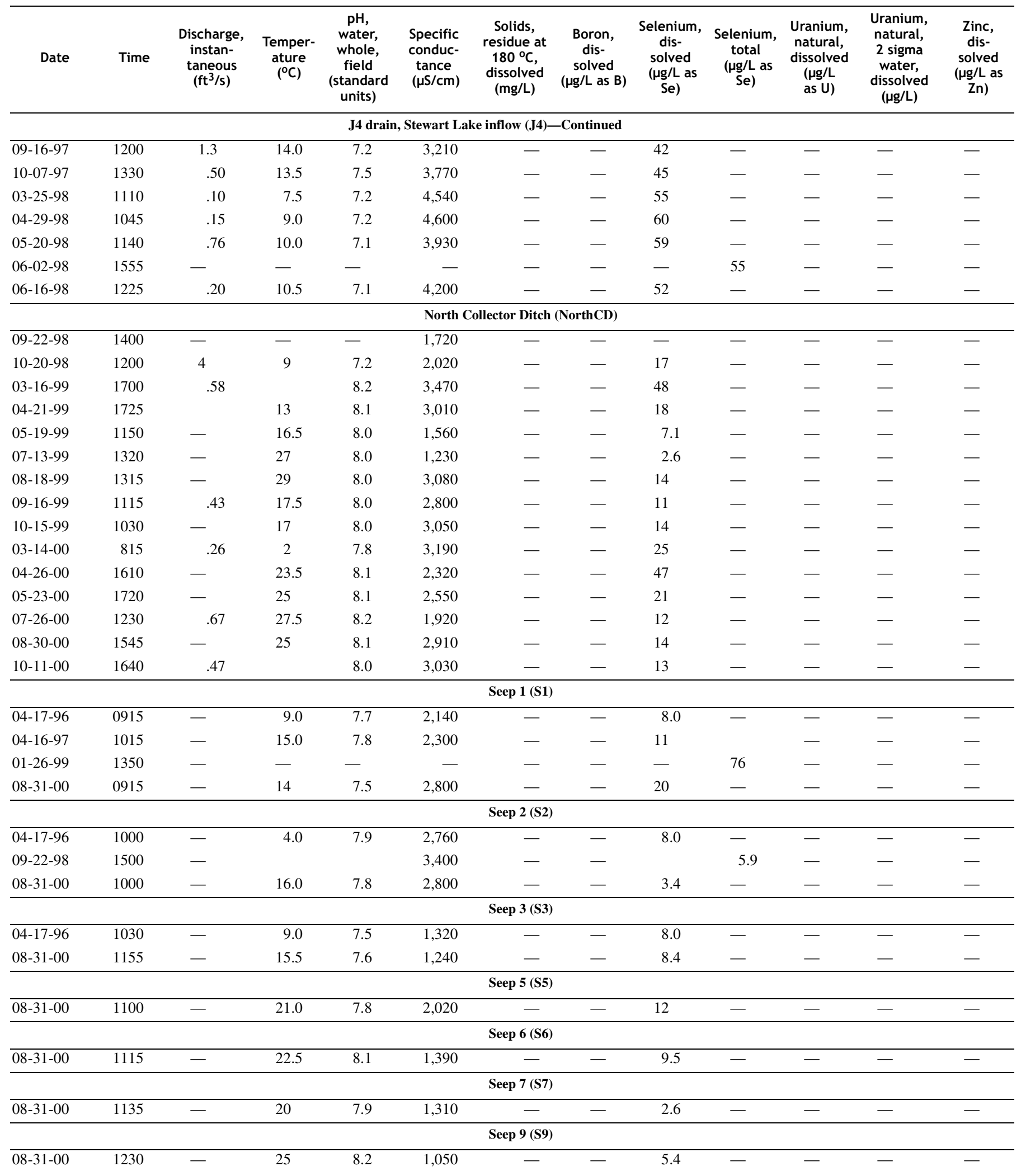


Table 7. Discharge, physical properties, and selected chemical analyses of surface-water inflow to Stewart Lake near Jensen, Utah, water years 1991 2000-Continued

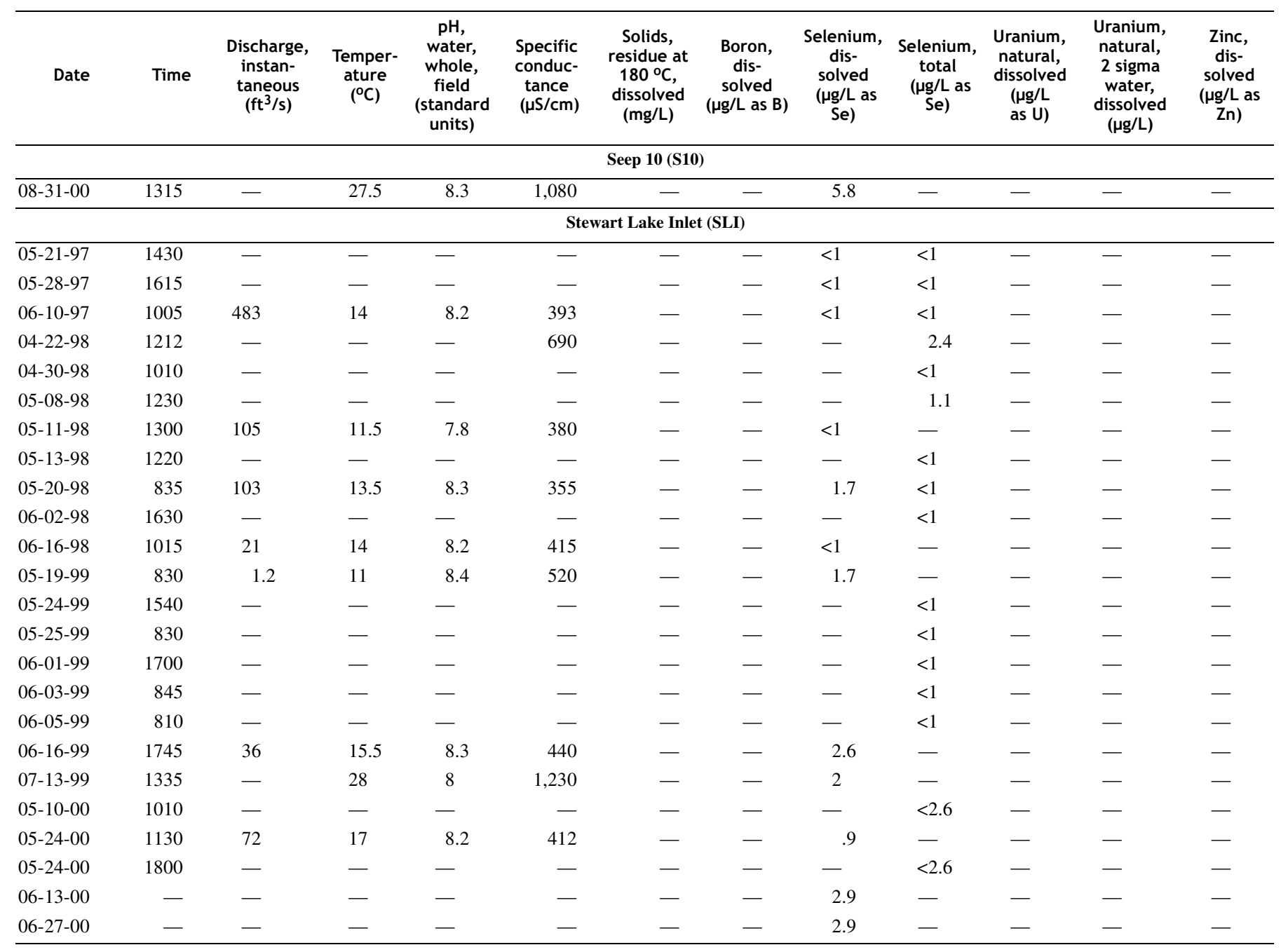


Table 8. Nitrogen concentration of surface-water inflow to Stewart Lake near Jensen, Utah, 1991 and 1994

[Data from the U.S. Geological Survey; mg/L, milligrams per liter; —, no data]

\begin{tabular}{|c|c|c|c|c|c|c|c|c|c|c|c|}
\hline $\begin{array}{l}\text { Drain } \\
\text { name }\end{array}$ & Date & Time & $\begin{array}{l}\text { Nitrogen, } \\
\text { ammonia, } \\
\text { dissolved } \\
\text { (mg/L as } \mathrm{N} \text { ) }\end{array}$ & $\begin{array}{l}\text { Nitrogen, } \\
\text { ammonia, } \\
\text { dissolved } \\
\left(\mathrm{mg} / \mathrm{L} \text { as } \mathrm{NH}_{4}\right)\end{array}$ & $\begin{array}{l}\text { Nitrogen, } \\
\text { nitrate, } \\
\text { dissolved } \\
\text { (mg/L as } N)\end{array}$ & $\begin{array}{l}\text { Nitrogen, } \\
\text { nitrate, total } \\
(\mathrm{mg} / \mathrm{L} \text { as } \mathrm{N})\end{array}$ & $\begin{array}{c}\text { Nitrogen, } \\
\text { nitrate, } \\
\text { dissolved } \\
\left.\left(\mathrm{mg}^{2} \mathrm{~L} \text { as NO}\right)_{3}\right)\end{array}$ & $\begin{array}{c}\text { Nitrogen, } \\
\text { nitrite, } \\
\text { dissolved } \\
\text { (mg/L as } \mathrm{N})\end{array}$ & $\begin{array}{c}\text { Nitrogen, } \\
\text { nitrite, } \\
\text { dissolved } \\
\left(\mathrm{mg} / \mathrm{L} \text { as } \mathrm{NO}_{2}\right)\end{array}$ & $\begin{array}{c}\text { Nitrogen, } \\
\mathrm{NO}_{2}+\mathrm{NO}_{3} \\
\text { total } \\
\text { (mg/L as } \mathrm{N} \text { ) }\end{array}$ & $\begin{array}{c}\text { Nitrogen, } \\
\mathrm{NO}_{2}+\mathrm{NO}_{3} \\
\text { dissolved } \\
\text { (mg/L as } \mathrm{N})\end{array}$ \\
\hline \multirow[t]{2}{*}{$\mathrm{J} 1$} & $08-28-91$ & 1400 & - & - & 3.5 & 3.5 & 15 & .01 & .03 & 3.5 & 3.5 \\
\hline & $03-17-94$ & 1110 & .02 & .03 & - & - & - & - & - & - & - \\
\hline J1A & 03-17-94 & 1100 & .02 & .03 & - & - & - & - & - & - & - \\
\hline \multirow[t]{2}{*}{$\mathrm{J} 2$} & $08-27-91$ & 1640 & - & - & .25 & .25 & 1.1 & .02 & .07 & .27 & .27 \\
\hline & $03-17-94$ & 1220 & .04 & .05 & - & - & - & - & - & - & - \\
\hline \multirow[t]{2}{*}{$\mathrm{J} 3$} & $08-28-91$ & 1530 & - & - & 3.9 & 3.9 & 17 & .03 & .10 & 3.9 & 3.9 \\
\hline & 03-17-94 & 1240 & .03 & .04 & - & - & - & - & - & - & - \\
\hline \multirow[t]{2}{*}{$\mathrm{J} 4$} & $08-28-91$ & 1600 & - & - & 4.2 & 4.2 & 19 & .01 & .03 & 4.2 & 4.2 \\
\hline & $03-17-94$ & 1400 & .03 & .04 & - & - & - & - & - & - & - \\
\hline
\end{tabular}

Table 9. Concentration of selected radiochemicals in water from irrigation drains J3 and J4 near Jensen, Utah, 1992

[Data from the U.S. Geological Survey; pCi/L, picocuries per liter; $\mu \mathrm{g} / \mathrm{L}$, micrograms per liter]

\begin{tabular}{|c|c|c|c|c|c|c|c|c|c|c|}
\hline Drain name & Date & Time & $\begin{array}{l}\text { Alpha radio, } \\
\text { dissolved as } \\
\text { Th-230 } \\
\text { (pCi/L) }\end{array}$ & $\begin{array}{c}\text { Gross alpha, } \\
\text { dissolved as } \\
\text { Nat U } \\
(\mu \mathrm{g} / \mathrm{L})\end{array}$ & $\begin{array}{c}\text { Alpha, count, } 2 \\
\text { sigma } \\
\text { dissolved as } \\
\text { Nat U } \\
(\mu \mathrm{g} / \mathrm{L})\end{array}$ & $\begin{array}{c}\text { Alpha count, } 2 \\
\text { sigma } \\
\text { dissolved as } \\
\text { Th-230 } \\
\text { (pCi/L) }\end{array}$ & $\begin{array}{l}\text { Gross beta, } \\
\text { dissolved } \\
\text { (pCi/L as } \\
\mathrm{Sr} / \mathrm{Y}-90 \text { ) }\end{array}$ & $\begin{array}{c}\text { Beta, } \\
2 \text { sigma } \\
\text { dissolved as } \\
\text { Sr90/Y90 } \\
(\mathrm{pCi} / \mathrm{L})\end{array}$ & $\begin{array}{l}\text { Gross beta, } \\
\text { dissolved } \\
\text { (pCi/L as } \\
\text { Cs-137) }\end{array}$ & $\begin{array}{c}\text { Beta, } \\
2 \text { sigma } \\
\text { dissolved as } \\
\text { Cs-137 } \\
(\mathrm{pCi} / \mathrm{L})\end{array}$ \\
\hline$\overline{J 3}$ & $07-15-92$ & 1010 & 36 & 49 & 6.0 & 4.4 & 26 & 6.5 & 35 & 8.8 \\
\hline $\mathrm{J} 4$ & $07-15-92$ & 0940 & 26 & 38 & 5.0 & 3.3 & 25 & 6.2 & 32 & 8.2 \\
\hline
\end{tabular}


Table 10. Physical properties and chemical analyses of water from wells in the Stewart Lake Waterfowl Management Area near Jensen, Utah, water years 1996-2000

[Data from the U.S. Geological Survey; ${ }^{\circ} \mathrm{C}$, degrees Celsius; $\mu \mathrm{S} / \mathrm{cm}$, microsiemens per centimeter at $25^{\circ} \mathrm{C}$; ANC, acid neutralizing capacity; mg/L, milligrams per liter; $\mu \mathrm{g} / \mathrm{L}$, micrograms per liter; —, not determined; <, less than; E, estimated]

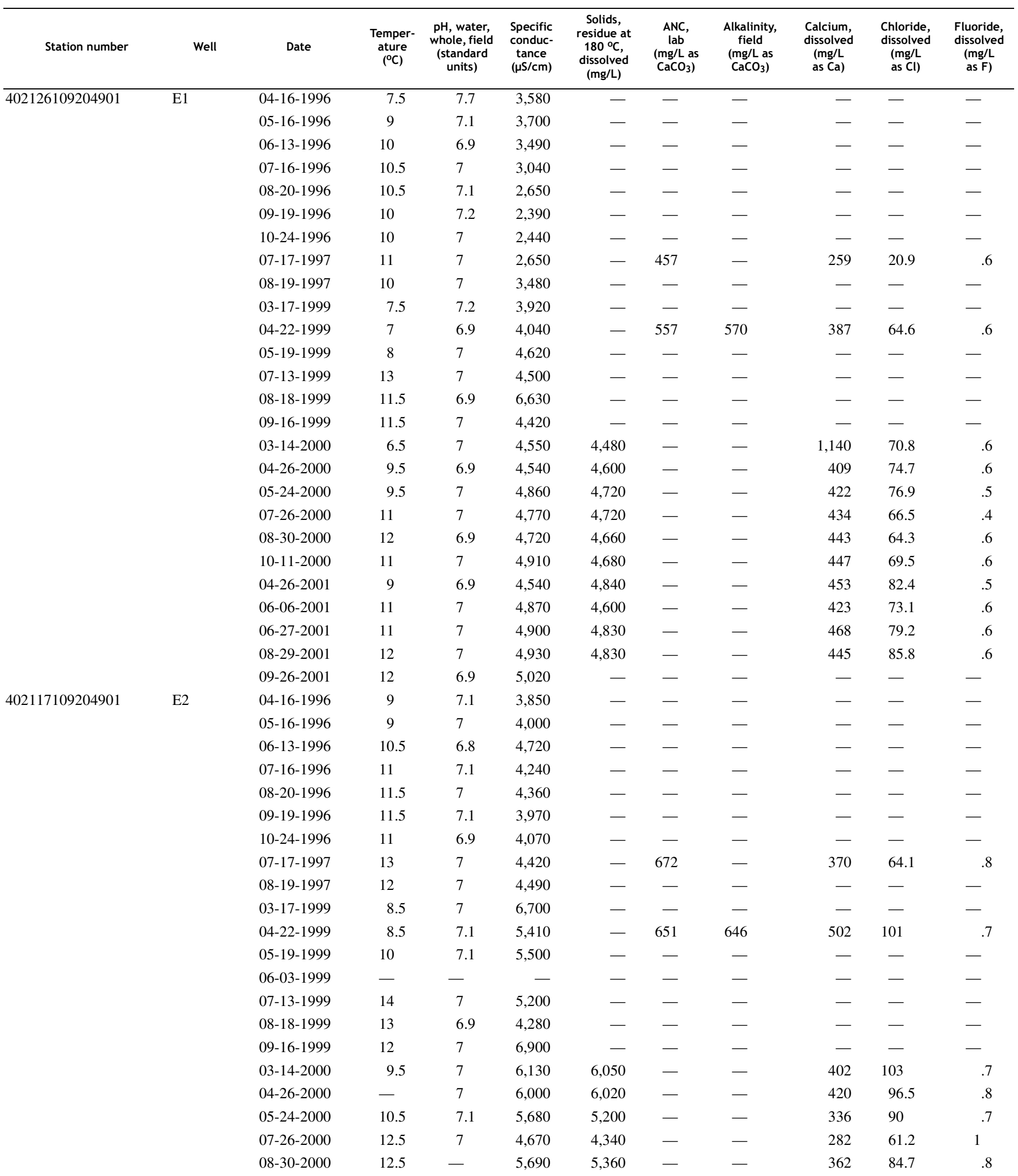


Table 10. Physical properties and chemical analyses of water from wells in the Stewart Lake Waterfowl Management Area near Jensen, Utah, water years, 1996-2000-Continued

\begin{tabular}{|c|c|c|c|c|c|c|c|c|c|c|c|c|}
\hline Station number & $\begin{array}{c}\text { Iron, } \\
\text { dissolved } \\
(\mu \mathrm{g} / \mathrm{L} \\
\text { as Fe) }\end{array}$ & $\begin{array}{c}\text { Magne- } \\
\text { sium, } \\
\text { dissolved } \\
(\mathrm{mg} / \mathrm{L} \text { as } \\
\mathrm{Mg})\end{array}$ & $\begin{array}{c}\text { Manga- } \\
\text { nese, } \\
\text { dissolved } \\
(\mu \mathrm{g} / \mathrm{L} \text { as } \mathrm{Mn})\end{array}$ & $\begin{array}{l}\text { Potas-sium, } \\
\text { dissolved } \\
\text { (mg/L as K) }\end{array}$ & $\begin{array}{c}\text { Silica, } \\
\text { dissolved } \\
(\mathrm{mg} / \mathrm{L} \text { as } \\
\left.\mathrm{SiO}_{2}\right)\end{array}$ & $\begin{array}{l}\text { Sodium, } \\
\text { dissolved } \\
(\mathrm{mg} / \mathrm{L} \text { as } \\
\mathrm{Na})\end{array}$ & $\begin{array}{l}\text { Sulfate, } \\
\text { dissolved } \\
\text { (mg/L as } \\
\left.\mathrm{SO}_{4}\right)\end{array}$ & $\begin{array}{c}\text { Arsenic, } \\
\text { dissolved } \\
(\mu \mathrm{g} / \mathrm{L} \\
\text { as As) }\end{array}$ & $\begin{array}{c}\text { Nitrogen } \\
\mathrm{NO}_{2}+\mathrm{NO}_{3} \\
\text { dis- } \\
\text { solved } \\
(\mathrm{mg} / \mathrm{L} \text { as } \mathrm{N})\end{array}$ & \multicolumn{2}{|c|}{$\begin{array}{c}\text { Selenium, } \\
\text { dissolved } \\
\text { ( } \mu \mathrm{g} / \mathrm{L} \text { as } \mathrm{Se} \text { ) }\end{array}$} & $\begin{array}{c}\text { Selenium, } \\
\text { total } \\
(\mu \mathrm{g} / \mathrm{L} \\
\text { as Se) }\end{array}$ \\
\hline \multirow[t]{26}{*}{402126109204901} & - & - & - & - & - & - & - & - & - & \multicolumn{2}{|l|}{$<1$} & - \\
\hline & - & - & - & - & - & - & - & - & - & \multicolumn{2}{|l|}{$<1$} & - \\
\hline & - & - & - & - & - & - & - & - & - & \multicolumn{2}{|l|}{$<1$} & - \\
\hline & - & - & - & - & - & - & - & - & - & \multicolumn{2}{|l|}{$<1$} & - \\
\hline & - & - & - & - & - & - & - & - & - & \multicolumn{2}{|l|}{$<1$} & - \\
\hline & - & - & - & - & - & - & - & - & - & \multicolumn{2}{|l|}{$<1$} & - \\
\hline & - & - & - & - & - & - & - & - & - & \multicolumn{2}{|l|}{$<1$} & - \\
\hline & - & 150 & - & 4.52 & 15.8 & 216 & 1,090 & - & - & \multicolumn{2}{|l|}{$<1$} & $<1$ \\
\hline & - & - & - & - & - & - & - & - & - & \multicolumn{2}{|l|}{$<1$} & - \\
\hline & - & - & - & - & - & - & - & - & - & \multicolumn{2}{|l|}{1.6} & - \\
\hline & 2,090 & 239 & 3,310 & 5.81 & 17.1 & 443 & 2,120 & - & - & \multicolumn{2}{|l|}{-} & - \\
\hline & - & - & - & - & - & - & - & - & - & \multicolumn{2}{|l|}{1.1} & - \\
\hline & - & - & - & - & - & - & - & - & - & \multicolumn{2}{|l|}{$<1$} & - \\
\hline & - & - & - & - & - & - & - & - & - & \multicolumn{2}{|l|}{2.4} & - \\
\hline & - & - & - & - & - & - & - & - & - & \multicolumn{2}{|l|}{$<2.4$} & - \\
\hline & 3,680 & 242 & 9,330 & 6.88 & 47.4 & 457 & 2,490 & - & $<.050$ & 1.9 & $\mathrm{E}$ & - \\
\hline & $<30$ & 154 & 1,570 & .58 & 19.9 & 1,600 & 2,500 & - & $<.050$ & \multicolumn{2}{|l|}{10} & - \\
\hline & 520 & 236 & 3,430 & 6.86 & 16.4 & 464 & 2,590 & - & $<.050$ & 1.2 & $\mathrm{E}$ & - \\
\hline & 90 & 259 & 3,860 & 8.35 & 18.3 & 511 & 2,560 & - & $<.050$ & 1.5 & E & - \\
\hline & 460 & 266 & 3,880 & 8.24 & 18.6 & 526 & 2,540 & - & $<.050$ & 1.6 & $\mathrm{E}$ & - \\
\hline & 730 & 249 & 3,730 & 8.12 & 18.3 & 486 & 2,590 & - & $<.047$ & 2.2 & $\mathrm{E}$ & - \\
\hline & 2,110 & 266 & 3,830 & 7.74 & 17.6 & 518 & 2,610 & - & $<.047$ & \multicolumn{2}{|l|}{$<2.4$} & - \\
\hline & $<30$ & 258 & 3,290 & 7.37 & 17.8 & 503 & 2,610 & - & $<.050$ & 1.3 & & - \\
\hline & 90 & 272 & 3,830 & 7.28 & 18.8 & 518 & 2,630 & - & $<.050$ & 1.7 & & - \\
\hline & 730 & 255 & 3,710 & 6.82 & 17.8 & 511 & 2,660 & - & $.007 \mathrm{E}$ & $<2$ & & - \\
\hline & - & - & - & - & - & - & - & - & $<.050$ & - & & - \\
\hline 402117109204901 & - & - & - & - & - & - & - & - & - & $<1$ & & - \\
\hline & - & - & - & - & - & - & - & - & - & $<1$ & & - \\
\hline & - & - & - & - & - & - & - & - & - & $<1$ & & - \\
\hline & - & - & - & - & - & - & - & - & - & - & & - \\
\hline & - & - & - & - & - & - & - & - & - & $<1$ & & - \\
\hline & - & - & - & - & - & - & - & - & - & $<1$ & & - \\
\hline & - & - & - & - & - & - & - & - & - & $<1$ & & - \\
\hline & - & 252 & - & 6.65 & 19.8 & 520 & 2,310 & - & - & $<1$ & & $<1$ \\
\hline & - & - & - & - & - & - & - & - & - & $<1$ & & - \\
\hline & - & - & - & - & - & - & - & - & - & 3.7 & & - \\
\hline & 830 & 358 & 2,900 & 8.56 & 14.3 & 767 & 3,080 & - & - & - & & - \\
\hline & - & - & - & - & - & - & - & - & - & $<1$ & & - \\
\hline & - & - & - & - & - & - & - & - & - & - & & $<1$ \\
\hline & - & - & - & - & - & - & - & - & - & $<1$ & & - \\
\hline & - & - & - & - & - & - & - & - & - & 1.4 & & - \\
\hline & - & - & - & - & - & - & - & - & - & $<2.4$ & & - \\
\hline & 2,540 & 321 & 3,680 & 8.78 & 19.4 & 814 & 3,450 & - & $<.050$ & 2.3 & E & - \\
\hline & 1,230 & 304 & 3,230 & 8.16 & 19.1 & 789 & 3,430 & 3 & $<.050$ & - & & - \\
\hline & 740 & 249 & 2,490 & 8.67 & 18 & 752 & 2,900 & - & $<.050$ & 1.6 & $\mathrm{E}$ & - \\
\hline & 410 & 228 & 2,570 & 7.79 & 19.3 & 641 & 2,400 & - & $<.050$ & $<2.4$ & & - \\
\hline & 960 & 285 & 3,210 & 8.53 & 19.9 & 778 & 2,970 & - & $<.050$ & 1.4 & $\mathrm{E}$ & - \\
\hline
\end{tabular}


Table 10. Physical properties and chemical analyses of water from wells in the Stewart Lake Waterfowl Management Area near Jensen, Utah, water years, 1996-2000-Continued

\begin{tabular}{|c|c|c|c|c|c|c|c|c|c|c|c|}
\hline Station number & Well & Date & $\begin{array}{l}\text { Temper- } \\
\text { ature } \\
\left({ }^{\circ} \mathrm{C}\right)\end{array}$ & $\begin{array}{l}\mathrm{pH}, \text { water, } \\
\text { whole, field } \\
\text { (standard } \\
\text { units) }\end{array}$ & $\begin{array}{c}\text { Specific } \\
\text { conduc- } \\
\text { tance } \\
(\mu \mathrm{S} / \mathrm{cm})\end{array}$ & $\begin{array}{l}\text { Solids, } \\
\text { residue at } \\
180^{\circ} \mathrm{C}, \\
\text { dissolved } \\
\text { (mg/L) }\end{array}$ & $\begin{array}{c}\mathrm{ANC}, \\
\mathrm{lab} \\
(\mathrm{mg} / \mathrm{L} \text { as } \\
\left.\mathrm{CaCO}_{3}\right)\end{array}$ & $\begin{array}{c}\text { Alkalinity, } \\
\text { field } \\
(\mathrm{mg} / \mathrm{L} \text { as } \\
\left.\mathrm{CaCO}_{3}\right)\end{array}$ & $\begin{array}{l}\text { Calcium, } \\
\text { dissolved } \\
\text { (mg/L } \\
\text { as Ca) }\end{array}$ & $\begin{array}{l}\text { Chloride, } \\
\text { dissolved } \\
\text { (mg/L } \\
\text { as Cl) }\end{array}$ & $\begin{array}{l}\text { Fluoride, } \\
\text { dissolved } \\
\text { (mg/L } \\
\text { as F) }\end{array}$ \\
\hline \multirow{6}{*}{$\begin{array}{c}402117109204901 \\
\text {-Continued }\end{array}$} & \multirow[t]{6}{*}{ E2 } & $10-11-2000$ & 12.5 & 7 & 5,500 & 5,130 & - & - & 350 & 85.7 & .7 \\
\hline & & 04-26-2001 & 10 & 7.1 & 4,530 & 4,430 & - & - & 296 & 68.5 & .8 \\
\hline & & 06-06-2001 & 11 & 7.1 & 4,830 & 4,380 & - & - & 279 & 66.2 & .8 \\
\hline & & 06-27-2001 & 12 & 7.2 & 4,770 & 4,220 & - & - & 277 & 70.1 & .8 \\
\hline & & 08-29-2001 & 12 & 7.2 & 4,190 & 3,560 & - & - & 218 & 59.4 & .9 \\
\hline & & 09-26-2001 & 12.5 & 7.1 & 4,030 & - & - & - & - & - & - \\
\hline \multirow[t]{27}{*}{402111109210701} & \multirow[t]{27}{*}{ E3 } & 04-16-1996 & 7.5 & 7.1 & 5,970 & - & - & - & - & - & - \\
\hline & & 05-16-1996 & 8.5 & 7 & 4,050 & - & - & - & - & - & - \\
\hline & & 06-13-1996 & 10.5 & 6.8 & 3,750 & - & - & - & - & - & - \\
\hline & & 07-16-1996 & 11 & 7.1 & 4,010 & - & - & - & - & - & - \\
\hline & & 08-20-1996 & 11.5 & 7.1 & 3,170 & - & - & - & - & - & - \\
\hline & & 09-19-1996 & 11.5 & 7.2 & 2,970 & - & - & - & - & - & - \\
\hline & & $10-24-1996$ & 11 & 7 & 3,030 & - & - & - & - & - & - \\
\hline & & 07-17-1997 & 13 & 7.1 & 3,060 & - & 534 & - & 245 & 36.7 & .9 \\
\hline & & 08-19-1997 & 12.5 & 7 & 3,260 & - & - & - & - & - & - \\
\hline & & 03-17-1999 & 8.5 & 7.1 & 3,520 & - & - & - & - & - & - \\
\hline & & 04-22-1999 & 8.5 & 7 & 3,240 & - & 582 & 654 & 288 & 43.9 & .9 \\
\hline & & 05-19-1999 & 9 & 7.1 & 3650 & - & - & - & - & - & - \\
\hline & & 06-03-1999 & - & - & - & - & - & - & - & - & - \\
\hline & & 07-13-1999 & 20 & 7 & 4,900 & - & - & - & - & - & - \\
\hline & & 08-18-1999 & 16 & 7 & 3,920 & - & - & - & - & - & - \\
\hline & & 09-16-1999 & 14.5 & 7 & 4,790 & - & - & - & - & - & - \\
\hline & & $03-14-2000$ & 9 & 7.1 & 4,110 & 3,860 & - & - & 291 & 50.5 & 1 \\
\hline & & $04-26-2000$ & 10.5 & 7.1 & 4,090 & 3,980 & - & - & 269 & 48 & 1 \\
\hline & & $05-24-2000$ & 10.5 & 7 & 4,150 & 4,730 & - & - & 309 & 47.8 & 1 \\
\hline & & $07-26-2000$ & 15.5 & 7 & 4,680 & 4,340 & - & - & 340 & 52.4 & 1.1 \\
\hline & & 08-30-2000 & 15 & 7 & 4,980 & 4,820 & - & - & 366 & 60.1 & 1 \\
\hline & & $10-11-2000$ & - & 7.1 & 4,370 & 3,990 & - & - & 330 & 56.5 & 1 \\
\hline & & 04-26-2001 & 10 & 7 & 4,050 & 4,230 & - & - & 348 & 52.5 & .9 \\
\hline & & 06-06-2001 & 12 & 7.1 & 4,730 & 4,560 & - & - & 365 & 52.1 & .9 \\
\hline & & $06-27-2001$ & 13 & 7.1 & 4,510 & - & - & - & - & - & - \\
\hline & & 08-29-2001 & 13 & 7 & 4,390 & 4,040 & - & - & 331 & 56.2 & 1.1 \\
\hline & & 09-26-2001 & 13.5 & 7 & 4,230 & - & - & - & - & - & - \\
\hline \multirow[t]{13}{*}{402111109215901} & \multirow[t]{13}{*}{ W1 } & 04-16-1996 & 7.5 & 7.1 & 7,780 & - & - & - & - & - & - \\
\hline & & 05-16-1996 & 8.5 & 7 & 7,940 & - & - & - & - & - & - \\
\hline & & 06-13-1996 & - & 6.8 & 7,920 & - & - & - & - & - & - \\
\hline & & 07-16-1996 & 9.5 & 7.1 & 7,910 & - & - & - & - & - & - \\
\hline & & 08-20-1996 & 11 & 7 & 8,080 & - & - & - & - & - & - \\
\hline & & 09-19-1996 & 10 & 7.1 & 7,700 & - & - & - & - & - & - \\
\hline & & $10-24-1996$ & 10.5 & 7 & 8,110 & - & - & - & - & - & - \\
\hline & & 08-19-1997 & - & 7.1 & 8,860 & - & - & - & - & - & - \\
\hline & & 03-17-1999 & 7.5 & 7 & 6,540 & - & - & - & - & - & - \\
\hline & & 04-22-1999 & 8.5 & 7 & 4,060 & - & 678 & 788 & 292 & 72.8 & .2 \\
\hline & & 05-20-1999 & 10 & 7 & 7,010 & - & - & - & - & - & - \\
\hline & & 07-13-1999 & 20 & 7 & 4,690 & - & - & - & - & - & - \\
\hline & & 08-18-1999 & 12.5 & 7 & 6,810 & - & - & - & - & - & - \\
\hline
\end{tabular}


Table 10. Physical properties and chemical analyses of water from wells in the Stewart Lake Waterfowl Management Area near Jensen, Utah, water years, 1996-2000-Continued

\begin{tabular}{|c|c|c|c|c|c|c|c|c|c|c|c|c|}
\hline Station number & $\begin{array}{l}\text { Iron, } \\
\text { dissolved } \\
(\mu \mathrm{g} / \mathrm{L} \\
\text { as Fe })\end{array}$ & $\begin{array}{l}\text { Magne- } \\
\text { sium, } \\
\text { dissolved } \\
(\mathrm{mg} / \mathrm{L} \text { as } \\
\mathrm{Mg})\end{array}$ & $\begin{array}{c}\text { Manga- } \\
\text { nese, } \\
\text { dissolved } \\
(\mu \mathrm{g} / \mathrm{L} \text { as } \mathrm{Mn})\end{array}$ & $\begin{array}{l}\text { Potas-sium, } \\
\text { dissolved } \\
\text { (mg/L as K) }\end{array}$ & $\begin{array}{l}\text { Silica, } \\
\text { dissolved } \\
(\mathrm{mg} / \mathrm{L} \text { as } \\
\left.\mathrm{SiO}_{2}\right)\end{array}$ & $\begin{array}{l}\text { Sodium, } \\
\text { dissolved } \\
\text { (mg/L as } \\
\mathrm{Na})\end{array}$ & $\begin{array}{l}\text { Sulfate, } \\
\text { dissolved } \\
(\mathrm{mg} / \mathrm{L} \text { as } \\
\left.\mathrm{SO}_{4}\right)\end{array}$ & $\begin{array}{c}\text { Arsenic, } \\
\text { dissolved } \\
(\mu \mathrm{g} / \mathrm{L} \\
\text { as As) }\end{array}$ & $\begin{array}{c}\text { Nitrogen } \\
\mathrm{NO}_{2}+\mathrm{NO}_{3} \\
\text { dis- } \\
\text { solved } \\
\text { (mg/L as } \mathrm{N} \text { ) }\end{array}$ & \multicolumn{2}{|c|}{$\begin{array}{l}\text { Selenium, } \\
\text { dissolved } \\
(\mu \mathrm{g} / \mathrm{L} \text { as } \mathrm{Se})\end{array}$} & $\begin{array}{l}\text { Selenium, } \\
\text { total } \\
(\mu \mathrm{g} / \mathrm{L} \\
\text { as Se) }\end{array}$ \\
\hline \multirow{6}{*}{$\begin{array}{c}02117109204901 \\
\text { —Continued }\end{array}$} & 1,920 & 255 & 2,970 & 8.15 & 20.5 & 764 & 2,860 & - & $<.047$ & 1.4 & $E$ & - \\
\hline & 1,270 & 216 & 2,450 & 7.32 & 18.8 & 698 & 2,410 & - & $<.047$ & $<2.4$ & & - \\
\hline & $<30$ & 213 & 1,840 & 6.78 & 17.5 & 659 & 2,430 & - & $<.050$ & 1.4 & $\mathrm{E}$ & - \\
\hline & E30 & 206 & 2,190 & 6.95 & 19.6 & 660 & 2,300 & - & $<.050$ & 1.3 & $\mathrm{E}$ & - \\
\hline & 310 & 160 & 1,790 & 7.09 & 18.2 & 599 & 1,960 & - & $.006 \mathrm{E}$ & $<2.0$ & & - \\
\hline & - & - & - & - & - & - & - & - & $.023 \mathrm{E}$ & - & & - \\
\hline \multirow[t]{27}{*}{402111109210701} & - & - & - & - & - & - & - & - & - & $<1$ & & - \\
\hline & - & - & - & - & - & - & - & - & - & $<1$ & & - \\
\hline & - & - & - & - & - & - & - & - & - & $<1$ & & - \\
\hline & - & - & - & - & - & - & - & - & - & $<1$ & & - \\
\hline & - & - & - & - & - & - & - & - & - & $<1$ & & - \\
\hline & - & - & - & - & - & - & - & - & - & $<1$ & & - \\
\hline & - & - & - & - & - & - & - & - & - & $<1$ & & - \\
\hline & - & 166 & - & 5.58 & 26.3 & 340 & 1,340 & - & - & $<1$ & & $<1$ \\
\hline & - & - & - & - & - & - & - & - & - & $<1$ & & - \\
\hline & - & - & - & - & - & - & - & - & - & 1.7 & & - \\
\hline & 280 & 197 & 3,120 & 3.45 & 22.8 & 351 & 1,530 & - & - & - & & - \\
\hline & - & - & - & - & - & - & - & - & - & 1.1 & & - \\
\hline & - & - & - & - & - & - & - & - & - & - & & $<1$ \\
\hline & - & - & - & - & - & - & - & - & - & 1.4 & & - \\
\hline & - & - & - & - & - & - & - & - & - & $<1$ & & - \\
\hline & - & - & - & - & - & - & - & - & - & $<2.4$ & & - \\
\hline & 3,340 & 231 & 2,730 & 7.44 & 22.9 & 417 & 2,050 & - & $<.050$ & 1.7 & E & \\
\hline & 910 & 197 & 2,560 & 6.05 & 20.6 & 362 & 2,100 & - & $<.050$ & $<2.4$ & & - \\
\hline & 1,570 & 219 & 2,960 & 9.29 & 24.7 & 408 & 1,970 & - & $<.050$ & $<2.4$ & & - \\
\hline & 1,660 & 254 & 3,370 & 9.8 & 22.7 & 497 & 2,310 & - & $<.050$ & $<2.4$ & & - \\
\hline & 1,300 & 298 & 3,560 & 10.8 & 24.1 & 585 & 2,640 & - & $<.050$ & 1.9 & $\mathrm{E}$ & - \\
\hline & 1,970 & 244 & 3,040 & 10.2 & 25.1 & 475 & 2,190 & - & $<.047$ & $<2.4$ & & - \\
\hline & 2,160 & 253 & 2,850 & 12.6 & 23 & 449 & 2,170 & - & $<.047$ & $<2.4$ & & - \\
\hline & $<30$ & 278 & 1,650 & 13.7 & 17.4 & 496 & 2,490 & - & $<.050$ & 2.1 & E & - \\
\hline & - & - & - & - & - & - & - & - & - & - & & - \\
\hline & 1,790 & 239 & 2,670 & 11.3 & 25.4 & 461 & 2,120 & - & $.005 \mathrm{E}$ & $<2$ & & - \\
\hline & - & - & - & - & - & - & - & - & - & - & & - \\
\hline \multirow[t]{13}{*}{402111109215901} & - & - & - & - & - & - & - & - & - & $<1$ & & - \\
\hline & - & - & - & - & - & - & - & - & - & $<1$ & & - \\
\hline & - & - & - & - & - & - & - & - & - & $<1$ & & - \\
\hline & - & - & - & - & - & - & - & - & - & $<1$ & & - \\
\hline & - & - & - & - & - & - & - & - & - & $<1$ & & - \\
\hline & - & - & - & - & - & - & - & - & - & $<1$ & & - \\
\hline & - & - & - & - & - & - & - & - & - & $<1$ & & - \\
\hline & - & - & - & - & - & - & - & - & - & 1.1 & & - \\
\hline & - & - & - & - & - & - & - & - & - & 12 & & - \\
\hline & 20,200 & 240 & 3,130 & 4.47 & 23.3 & 513 & 1,910 & - & - & - & & - \\
\hline & - & - & - & - & - & - & - & - & - & 4.8 & & - \\
\hline & - & - & - & - & - & - & - & - & - & 1.7 & & - \\
\hline & - & - & - & - & - & - & - & - & - & 2.3 & & - \\
\hline
\end{tabular}


Table 10. Physical properties and chemical analyses of water from wells in the Stewart Lake Waterfowl Management Area near Jensen, Utah, water years, 1996-2000-Continued

\begin{tabular}{|c|c|c|c|c|c|c|c|c|c|c|c|}
\hline Station number & Well & Date & $\begin{array}{l}\text { Temper- } \\
\text { ature } \\
\left({ }^{\circ} \mathrm{C}\right)\end{array}$ & $\begin{array}{l}\mathrm{pH}, \text { water, } \\
\text { whole, field } \\
\text { (standard } \\
\text { units) }\end{array}$ & $\begin{array}{c}\text { Specific } \\
\text { conduc- } \\
\text { tance } \\
(\mu \mathrm{S} / \mathrm{cm})\end{array}$ & $\begin{array}{l}\text { Solids, } \\
\text { residue at } \\
180^{\circ} \mathrm{C}, \\
\text { dissolved } \\
\text { (mg/L) }\end{array}$ & $\begin{array}{c}\mathrm{ANC}, \\
\mathrm{lab} \\
(\mathrm{mg} / \mathrm{L} \text { as } \\
\left.\mathrm{CaCO}_{3}\right)\end{array}$ & $\begin{array}{l}\text { Alkalinity, } \\
\text { field } \\
(\mathrm{mg} / \mathrm{L} \text { as } \\
\left.\mathrm{CaCO}_{3}\right)\end{array}$ & $\begin{array}{l}\text { Calcium, } \\
\text { dissolved } \\
\text { (mg/L } \\
\text { as Ca) }\end{array}$ & $\begin{array}{l}\text { Chloride, } \\
\text { dissolved } \\
\text { (mg/L } \\
\text { as Cl) }\end{array}$ & $\begin{array}{l}\text { Fluoride, } \\
\text { dissolved } \\
\text { (mg/L } \\
\text { as F) }\end{array}$ \\
\hline \multirow{12}{*}{$\begin{array}{c}402111109215901 \\
\text {-Continued }\end{array}$} & \multirow[t]{12}{*}{ W1 } & 09-16-1999 & 12 & 7 & 6,780 & - & - & - & - & - & - \\
\hline & & 03-14-2000 & 6 & 7.1 & 6,760 & 6,580 & - & - & 360 & 134 & .7 \\
\hline & & 04-26-2000 & 8.5 & 7 & 6,660 & 6,520 & - & - & 302 & 130 & .7 \\
\hline & & 05-24-2000 & 9.5 & 7 & 6,950 & 6,570 & - & - & 377 & 135 & .7 \\
\hline & & $07-26-2000$ & - & 7 & 6,840 & 6,550 & - & - & 423 & 138 & 6 \\
\hline & & 08-30-2000 & 12 & 7 & 6,720 & 6,420 & - & - & 413 & 138 & .7 \\
\hline & & $10-11-2000$ & 11 & 7 & 6,990 & 6,510 & - & - & 428 & 148 & .7 \\
\hline & & 04-26-2001 & 11 & 7 & 6,690 & 7,110 & - & - & 427 & 175 & .7 \\
\hline & & 06-07-2001 & 10 & 7.1 & 7,480 & 7,300 & - & - & 416 & 194 & .5 \\
\hline & & 06-28/2001 & 10 & 7.1 & 7,140 & 7,030 & - & - & 431 & 174 & .4 \\
\hline & & 08-29-2001 & 10.5 & 6.9 & 7,390 & 7,050 & - & - & 439 & 191 & .7 \\
\hline & & 09-26-2001 & 10.5 & 6.9 & 7,250 & - & - & - & - & - & - \\
\hline \multirow[t]{26}{*}{402106109220601} & \multirow[t]{26}{*}{ W2 } & 04-16-1996 & 8 & 7 & 8,910 & - & - & - & - & - & - \\
\hline & & 05-16-1996 & 9 & 6.9 & 8,720 & - & - & - & - & - & - \\
\hline & & 06-13-1996 & 10 & 6.9 & 6,540 & - & - & - & - & - & - \\
\hline & & 07-16-1996 & 10 & 7.1 & 8,100 & - & - & - & - & - & - \\
\hline & & 08-20-1996 & 12 & 7 & 8,330 & - & - & - & - & - & - \\
\hline & & 09-19-1996 & 10.5 & 7.1 & 8,160 & - & - & - & - & - & - \\
\hline & & 10-24-1996 & 10 & 6.9 & 8,200 & - & - & - & - & - & - \\
\hline & & 08-19-1997 & - & 7.3 & 2,640 & - & - & - & - & - & - \\
\hline & & 03-17-1999 & 8.5 & 7.1 & 7,780 & - & - & - & - & - & - \\
\hline & & 04-22-1999 & 8 & 7 & 7,500 & - & 716 & 760 & 507 & 320 & .4 \\
\hline & & 05-20-1999 & 9.5 & 7.1 & 5,400 & - & - & - & - & - & - \\
\hline & & 07-13-1999 & 12.5 & 7 & 7,500 & - & - & - & - & - & - \\
\hline & & 08-18-1999 & 11.5 & 7 & 6,820 & - & - & - & - & - & - \\
\hline & & 09-16-1999 & 11 & 7 & 7,380 & - & - & - & - & - & - \\
\hline & & $03-14-2000$ & 7.5 & 7 & 7,560 & 7,370 & - & - & 380 & 257 & .4 \\
\hline & & 04-26-2000 & 9 & 7.1 & 7,490 & 7,260 & - & - & 400 & 235 & .5 \\
\hline & & 05-24-2000 & 10 & 7 & 7,830 & 7,410 & - & - & 403 & 243 & .4 \\
\hline & & $07-26-2000$ & - & 7 & 7,670 & 7,270 & - & - & 418 & 218 & .4 \\
\hline & & $08-30-2000$ & 10 & 7 & 7,530 & 7,240 & - & - & 425 & 220 & .4 \\
\hline & & $10-11-2000$ & 10 & 7 & 7,650 & 6,970 & - & - & 432 & 224 & .2 \\
\hline & & $04-26-2001$ & 10.5 & 7 & 7,020 & 7,320 & - & - & 419 & 212 & .4 \\
\hline & & 06-07-2001 & 10 & 7 & 7,380 & 6,890 & - & - & 414 & 201 & .4 \\
\hline & & $06-28-2001$ & 10 & 7 & 7,240 & 6,660 & - & - & 415 & 197 & .5 \\
\hline & & 08-29-2001 & 10 & 7 & 7,600 & 7,200 & - & - & 410 & 216 & .5 \\
\hline & & 08-29-2001 & 10 & 7 & 7,600 & - & - & - & - & - & - \\
\hline & & 09-26-2001 & 10 & 7 & 7,570 & - & - & - & - & - & - \\
\hline \multirow[t]{9}{*}{402056109220301} & \multirow[t]{9}{*}{ W3 } & 04-16-1996 & 5.5 & 7.4 & 8,110 & - & - & - & - & - & - \\
\hline & & 05-16-1996 & 8 & 7 & 7,420 & - & - & - & - & - & - \\
\hline & & 06-13-1996 & 9 & 6.8 & 7,530 & - & - & - & - & - & - \\
\hline & & 07-16-1996 & 10 & 6.9 & 8,070 & - & - & - & - & - & - \\
\hline & & 08-20-1996 & 11 & 7 & 7,560 & - & - & - & - & - & - \\
\hline & & 09-19-1996 & 12 & 7 & 7,180 & - & - & - & - & - & - \\
\hline & & $10-24-1996$ & 10 & 7 & 6,950 & - & - & - & - & - & - \\
\hline & & 07-17-1997 & 11 & 7 & 6,000 & - & 722 & - & 387 & 178 & .3 \\
\hline & & 03-17-1999 & 8 & 7.1 & 4,230 & - & - & - & - & - & - \\
\hline
\end{tabular}


Table 10. Physical properties and chemical analyses of water from wells in the Stewart Lake Waterfowl Management Area near Jensen, Utah, water years, 1996-2000-Continued

\begin{tabular}{|c|c|c|c|c|c|c|c|c|c|c|c|c|}
\hline Station number & $\begin{array}{l}\text { Iron, } \\
\text { dissolved } \\
(\mu \mathrm{g} / \mathrm{L} \\
\text { as Fe })\end{array}$ & $\begin{array}{l}\text { Magne- } \\
\text { sium, } \\
\text { dissolved } \\
(\mathrm{mg} / \mathrm{L} \text { as } \\
\mathrm{Mg})\end{array}$ & $\begin{array}{c}\text { Manga- } \\
\text { nese, } \\
\text { dissolved } \\
(\mu \mathrm{g} / \mathrm{L} \text { as } \mathrm{Mn})\end{array}$ & $\begin{array}{l}\text { Potas-sium, } \\
\text { dissolved } \\
\text { (mg/L as K) }\end{array}$ & $\begin{array}{l}\text { Silica, } \\
\text { dissolved } \\
(\mathrm{mg} / \mathrm{L} \text { as } \\
\left.\mathrm{SiO}_{2}\right)\end{array}$ & $\begin{array}{c}\text { Sodium, } \\
\text { dissolved } \\
(\mathrm{mg} / \mathrm{L} \text { as } \\
\mathrm{Na})\end{array}$ & $\begin{array}{l}\text { Sulfate, } \\
\text { dissolved } \\
(\mathrm{mg} / \mathrm{L} \text { as } \\
\left.\mathrm{SO}_{4}\right)\end{array}$ & $\begin{array}{c}\text { Arsenic, } \\
\text { dissolved } \\
(\mu \mathrm{g} / \mathrm{L} \\
\text { as As) }\end{array}$ & $\begin{array}{c}\text { Nitrogen } \\
\mathrm{NO}_{2}+\mathrm{NO}_{3} \\
\text { dis- } \\
\text { solved } \\
\text { (mg/L as } \mathrm{N} \text { ) }\end{array}$ & \multicolumn{2}{|c|}{$\begin{array}{l}\text { Selenium, } \\
\text { dissolved } \\
\text { ( } \mu \mathrm{g} / \mathrm{L} \text { as } \mathrm{Se})\end{array}$} & $\begin{array}{l}\text { Selenium, } \\
\text { total } \\
(\mu \mathrm{g} / \mathrm{L} \\
\text { as Se) }\end{array}$ \\
\hline \multirow{12}{*}{$\begin{array}{c}402111109215901 \\
\text { —Continued }\end{array}$} & - & - & - & - & - & - & - & - & - & $<2.4$ & & - \\
\hline & 930 & 327 & 3,060 & 7.11 & 23.6 & 906 & 3,570 & - & $<.050$ & 2.7 & & - \\
\hline & 140 & 258 & 2,480 & 5.11 & 21.4 & 721 & 3,520 & - & $<.050$ & 2.4 & $\mathrm{E}$ & - \\
\hline & 330 & 329 & 3,220 & 7.53 & 25.4 & 957 & 3,580 & - & $<.050$ & 2.1 & $\mathrm{E}$ & - \\
\hline & 1,790 & 346 & 3,570 & 10.7 & 24.3 & 935 & 3,490 & - & $<.050$ & 2.0 & $\mathrm{E}$ & - \\
\hline & 860 & 351 & 3,360 & 10.2 & 25.8 & 973 & 3,470 & - & $<.050$ & 3.1 & & - \\
\hline & 1,990 & 363 & 3,410 & 8.77 & 27.8 & 973 & 3,550 & - & $<.047$ & 3.0 & & - \\
\hline & 280 & 363 & 3,490 & 7.96 & 26.8 & 1,040 & 3,650 & - & $<.047$ & 1.5 & $\mathrm{E}$ & - \\
\hline & $<50$ & 396 & 1,480 & 10.8 & 20.3 & 1,060 & 3,950 & - & .084 & 34 & & - \\
\hline & $<10$ & 376 & 3,040 & 8.41 & 24.9 & 1,030 & 3,700 & - & $<.050$ & 4.7 & & - \\
\hline & 660 & 364 & 3,390 & 9.4 & 27.3 & 1,060 & 3,720 & - & $.007 \mathrm{E}$ & 2.6 & & - \\
\hline & - & - & - & - & - & - & - & - & $.027 \mathrm{E}$ & - & & - \\
\hline \multirow[t]{26}{*}{402106109220601} & - & - & - & - & - & - & - & - & - & $<1$ & & - \\
\hline & - & - & - & - & - & - & - & - & - & $<1$ & & - \\
\hline & - & - & - & - & - & - & - & - & - & 2.0 & & - \\
\hline & - & - & - & - & - & - & - & - & - & 1.0 & & - \\
\hline & - & - & - & - & - & - & - & - & - & $<1$ & & - \\
\hline & - & - & - & - & - & - & - & - & - & $<1$ & & - \\
\hline & - & - & - & - & - & - & - & - & - & $<1$ & & - \\
\hline & - & - & - & - & - & - & - & - & - & 1.3 & & - \\
\hline & - & - & - & - & - & - & - & - & - & 4.0 & & - \\
\hline & 16,100 & 406 & 2,410 & 9.17 & 17.7 & 1,300 & 4,110 & - & - & - & & - \\
\hline & - & - & - & - & - & - & - & - & - & 7.9 & & - \\
\hline & - & - & - & - & - & - & - & - & - & 2.3 & & - \\
\hline & - & - & - & - & - & - & - & - & - & 2.4 & & - \\
\hline & - & - & - & - & - & - & - & - & - & $<2.4$ & & - \\
\hline & 8,930 & 333 & 1,910 & 8.72 & 18 & 1,120 & 4,080 & - & $<.050$ & 2.7 & & - \\
\hline & 3,600 & 327 & 1,930 & 8.63 & 18.2 & 1,130 & 4,040 & - & .052 & 6.5 & & - \\
\hline & 7,710 & 330 & 2,000 & 8.16 & 18.7 & 1,160 & 4,060 & - & $<.050$ & 1.2 & E & - \\
\hline & 8,750 & 348 & 2,110 & 10.3 & 20.3 & 1,240 & 3,990 & - & $<.050$ & 1.6 & E & - \\
\hline & 1,710 & 356 & 2,090 & 9.72 & 19.8 & 1,230 & 3,990 & - & $<.050$ & 3.2 & & - \\
\hline & 11,800 & 352 & 2,060 & 10.1 & 21 & 1,140 & 3,910 & - & $<.047$ & 3.6 & & - \\
\hline & 6,110 & 335 & 2,030 & 8.82 & 20.3 & 1,160 & 3,880 & - & $<.047$ & 2.0 & $\mathrm{E}$ & - \\
\hline & $<50$ & 338 & 1,660 & 10.3 & 18.4 & 1,100 & 3,850 & - & $<.050$ & 1.8 & E & - \\
\hline & 950 & 340 & 1,830 & 8.62 & 20.4 & 1,150 & 3,850 & - & $<.050$ & 4.0 & & - \\
\hline & 4,020 & 333 & 1,970 & 10.4 & 20 & 1,190 & 3,940 & - & .018 & 4.1 & & - \\
\hline & - & - & - & - & - & - & - & - & - & 3.4 & & - \\
\hline & - & - & - & - & - & - & - & - & .035 & - & & - \\
\hline \multirow[t]{9}{*}{402056109220301} & - & - & - & - & - & - & - & - & - & 2.0 & & - \\
\hline & - & - & - & - & - & - & - & - & - & 1.0 & & - \\
\hline & - & - & - & - & - & - & - & - & - & 1.0 & & - \\
\hline & - & - & - & - & - & - & - & - & - & 1.0 & & - \\
\hline & - & - & - & - & - & - & - & - & - & $<1$ & & - \\
\hline & - & - & - & - & - & - & - & - & - & $<1$ & & - \\
\hline & - & - & - & - & - & - & - & - & - & $<1$ & & - \\
\hline & - & 308 & - & 6.54 & 17.7 & 904 & 2,420 & - & - & 1.8 & & 3.7 \\
\hline & - & - & - & - & - & - & - & - & - & 2.6 & & - \\
\hline
\end{tabular}


Table 10. Physical properties and chemical analyses of water from wells in the Stewart Lake Waterfowl Management Area near Jensen, Utah, water years, 1996-2000-Continued

\begin{tabular}{|c|c|c|c|c|c|c|c|c|c|c|c|}
\hline Station number & Well & Date & $\begin{array}{l}\text { Temper- } \\
\text { ature } \\
\left({ }^{\circ} \mathrm{C}\right)\end{array}$ & $\begin{array}{l}\mathrm{pH}, \text { water, } \\
\text { whole, field } \\
\text { (standard } \\
\text { units) }\end{array}$ & $\begin{array}{c}\text { Specific } \\
\text { conduc- } \\
\text { tance } \\
(\mu \mathrm{S} / \mathrm{cm})\end{array}$ & $\begin{array}{l}\text { Solids, } \\
\text { residue at } \\
180^{\circ} \mathrm{C}, \\
\text { dissolved } \\
\text { (mg/L) }\end{array}$ & $\begin{array}{c}\text { ANC, } \\
\text { lab } \\
(\mathrm{mg} / \mathrm{L} \text { as } \\
\left.\mathrm{CaCO}_{3}\right)\end{array}$ & $\begin{array}{c}\text { Alkalinity, } \\
\text { field } \\
(\mathrm{mg} / \mathrm{L} \text { as } \\
\left.\mathrm{CaCO}_{3}\right)\end{array}$ & $\begin{array}{l}\text { Calcium, } \\
\text { dissolved } \\
\text { (mg/L } \\
\text { as Ca) }\end{array}$ & $\begin{array}{c}\text { Chloride, } \\
\text { dissolved } \\
\text { (mg/L } \\
\text { as Cl) }\end{array}$ & $\begin{array}{c}\text { Fluoride, } \\
\text { dissolved } \\
\text { (mg/L } \\
\text { as F) }\end{array}$ \\
\hline \multirow{16}{*}{$\begin{array}{c}402056109220301 \\
\text {-Continued }\end{array}$} & \multirow[t]{16}{*}{ W3 } & 04-22-1999 & 7.5 & 7.1 & 6,380 & - & 836 & 752 & 474 & 151 & .6 \\
\hline & & 05-20-1999 & - & 7.1 & 4,160 & - & - & - & - & - & - \\
\hline & & 07-13-1999 & 19 & 7 & 5,320 & - & - & - & - & - & - \\
\hline & & 08-18-1999 & 14 & 6.9 & 5,680 & - & - & - & - & - & - \\
\hline & & 09-16-1999 & 11 & 7 & 4,400 & - & - & - & - & - & - \\
\hline & & $03-14-2000$ & 9.5 & 7.2 & 3,830 & 3,440 & - & - & 226 & 60.2 & .2 \\
\hline & & 04-26-2000 & 10.5 & 7.1 & 3,840 & 3,410 & - & - & 205 & 56.6 & .3 \\
\hline & & $05-24-2000$ & 11.5 & 7.1 & 4,020 & 3,520 & - & - & 230 & 57 & .3 \\
\hline & & $07-26-2000$ & 13 & 7 & 5,100 & 4,670 & - & - & 318 & 114 & .3 \\
\hline & & $08-30-2000$ & 12 & 7.1 & 3,850 & 3,260 & - & - & 243 & 59.7 & .3 \\
\hline & & $10-11-2000$ & 9 & 7.1 & 3,400 & 2,930 & - & - & 225 & 52.5 & .3 \\
\hline & & $04-26-2001$ & 10 & 7.5 & 3,600 & 3,240 & - & - & 234 & 55 & .3 \\
\hline & & 06-07-2001 & 10 & 7.2 & 3,410 & 2,900 & - & - & 231 & 51.4 & .4 \\
\hline & & $06-28 / 2001$ & 12 & 7.1 & 4,160 & 3,820 & - & - & 284 & 78.7 & .4 \\
\hline & & 08-29-2001 & 12.5 & 7 & 6,570 & 6,130 & - & - & 351 & 168 & .3 \\
\hline & & $09-26-2001$ & 10.5 & 7.1 & 3,760 & - & - & - & - & - & - \\
\hline
\end{tabular}


Table 10. Physical properties and chemical analyses of water from wells in the Stewart Lake Waterfowl Management Area near Jensen, Utah, water years, 1996-2000—Continued

\begin{tabular}{|c|c|c|c|c|c|c|c|c|c|c|c|c|}
\hline Station number & $\begin{array}{c}\text { Iron, } \\
\text { dissolved } \\
\text { ( } \mu \mathrm{g} / \mathrm{L} \\
\text { as Fe) }\end{array}$ & $\begin{array}{c}\text { Magne- } \\
\text { sium, } \\
\text { dissolved } \\
\text { (mg/L as } \\
\text { Mg) }\end{array}$ & $\begin{array}{l}\text { Manga- } \\
\text { nese, } \\
\text { dissolved } \\
(\mu \mathrm{g} / \mathrm{L} \text { as } \mathrm{Mn})\end{array}$ & $\begin{array}{l}\text { Potas-sium, } \\
\text { dissolved } \\
\text { (mg/L as K) }\end{array}$ & $\begin{array}{c}\text { Silica, } \\
\text { dissolved } \\
(\mathrm{mg} / \mathrm{L} \text { as } \\
\left.\mathrm{SiO}_{2}\right)\end{array}$ & $\begin{array}{l}\text { Sodium, } \\
\text { dissolved } \\
\text { (mg/L as } \\
\mathrm{Na})\end{array}$ & $\begin{array}{l}\text { Sulfate, } \\
\text { dissolved } \\
(\mathrm{mg} / \mathrm{L} \text { as } \\
\left.\mathrm{SO}_{4}\right)\end{array}$ & $\begin{array}{c}\text { Arsenic, } \\
\text { dissolved } \\
(\mu \mathrm{g} / \mathrm{L} \\
\text { as As })\end{array}$ & $\begin{array}{c}\text { Nitrogen } \\
\mathrm{NO}_{2}+\mathrm{NO}_{3} \\
\text { dis- } \\
\text { solved } \\
\text { (mg/L as } \mathrm{N})\end{array}$ & \multicolumn{2}{|c|}{$\begin{array}{l}\text { Selenium, } \\
\text { dissolved } \\
\text { ( } \mu \mathrm{g} / \mathrm{L} \text { as } \mathrm{Se})\end{array}$} & $\begin{array}{c}\text { Selenium, } \\
\text { total } \\
(\mu \mathrm{g} / \mathrm{L} \\
\text { as Se })\end{array}$ \\
\hline 402056109220301 & 740 & 381 & 3,480 & 7.53 & 21.9 & 1,030 & 3,450 & - & - & \multicolumn{2}{|l|}{-} & - \\
\hline —Continued & - & - & - & - & - & - & - & - & - & \multicolumn{2}{|l|}{1.8} & - \\
\hline & - & - & - & - & - & - & - & - & - & \multicolumn{2}{|l|}{$<1$} & - \\
\hline & - & - & - & - & - & - & - & - & - & \multicolumn{2}{|l|}{2.1} & - \\
\hline & - & - & - & - & - & - & - & - & - & \multicolumn{2}{|l|}{$<2.4$} & - \\
\hline & 13,800 & 197 & 2,650 & 4.06 & 24.5 & 441 & 1,730 & - & $<.050$ & 1.7 & $\mathrm{E}$ & - \\
\hline & 7,300 & 167 & 2,320 & 3.75 & 22.5 & 394 & 1,730 & - & $<.050$ & 1.5 & $\mathrm{E}$ & - \\
\hline & 13,400 & 193 & 2,670 & 4.19 & 24.5 & 451 & 1,800 & - & $<.050$ & 1.3 & $\mathrm{E}$ & - \\
\hline & 10,100 & 251 & 1,920 & 7.38 & 21.1 & 658 & 2,490 & - & $<.050$ & 2.3 & $\mathrm{E}$ & - \\
\hline & 10,900 & 199 & 2,400 & 5.37 & 24.6 & 458 & 1,680 & - & $<.050$ & \multicolumn{2}{|l|}{$<2.4$} & - \\
\hline & 15,400 & 180 & 2,740 & 3.96 & 26.7 & 388 & 1,420 & - & $<.047$ & \multicolumn{2}{|l|}{$<2.4$} & - \\
\hline & 5,790 & 200 & 2,380 & 4.32 & 24.6 & 435 & 1,580 & - & $<.047$ & \multicolumn{2}{|l|}{$<2.4$} & - \\
\hline & $<30$ & 175 & 2,100 & 5.07 & 20.6 & 369 & 1,440 & - & $.023 \mathrm{E}$ & 2.3 & E & - \\
\hline & 4,750 & 229 & 2,940 & 5.12 & 23.7 & 469 & 1,920 & - & $<.050$ & 1.6 & E & - \\
\hline & 18,500 & 360 & 2,680 & 6.21 & 23 & 913 & 3,280 & - & $.008 \mathrm{E}$ & 2.0 & E & - \\
\hline & - & - & - & - & - & - & - & - & $.028 \mathrm{E}$ & \multicolumn{2}{|l|}{-} & - \\
\hline
\end{tabular}


Table 11. Water levels for selected wells in the Stewart Lake Waterfowl Management Area near Jensen, Utah, water years 1996-2000

\begin{tabular}{|c|c|c|c|c|c|c|}
\hline Station number & Well & Date & $\begin{array}{l}\text { Land-surface } \\
\text { altitude } \\
\text { (feet) }\end{array}$ & $\begin{array}{l}\text { Well depth } \\
\text { below land } \\
\text { surface } \\
\text { (feet) }\end{array}$ & $\begin{array}{l}\text { Water level } \\
\text { below land } \\
\text { surface } \\
\text { (feet) }\end{array}$ & $\begin{array}{l}\text { Water-level } \\
\text { altitude } \\
\text { (feet) }\end{array}$ \\
\hline \multirow[t]{23}{*}{402126109204901} & \multirow[t]{23}{*}{ E1 } & $02-26-1996$ & $4,722.4$ & 24.8 & 2.30 & $4,720.10$ \\
\hline & & 04-16-1996 & $4,722.4$ & 24.8 & 1.98 & $4,720.42$ \\
\hline & & 05-16-1996 & $4,722.4$ & 24.8 & 1.14 & $4,721.26$ \\
\hline & & 06-13-1996 & $4,722.4$ & 24.8 & 1.84 & $4,720.56$ \\
\hline & & 07-16-1996 & $4,722.4$ & 24.8 & 2.81 & $4,719.59$ \\
\hline & & 08-20-1996 & $4,722.4$ & 24.8 & 2.92 & $4,719.48$ \\
\hline & & 09-19-1996 & $4,722.4$ & 24.8 & 2.50 & $4,719.90$ \\
\hline & & $10-24-1996$ & $4,722.4$ & 24.8 & 2.75 & $4,719.65$ \\
\hline & & 07-17-1997 & $4,722.4$ & 24.8 & 3.30 & $4,719.10$ \\
\hline & & 08-19-1997 & $4,722.4$ & 24.8 & 4.34 & $4,718.06$ \\
\hline & & 09-16-1997 & $4,722.4$ & 24.8 & 2.91 & $4,719.49$ \\
\hline & & 03-17-1999 & $4,722.4$ & 24.8 & 4.11 & $4,718.29$ \\
\hline & & 04-21-1999 & $4,722.4$ & 24.8 & 3.96 & $4,718.44$ \\
\hline & & 05-19-1999 & $4,722.4$ & 24.8 & 3.18 & $4,719.22$ \\
\hline & & 07-13-1999 & $4,722.4$ & 24.8 & 1.24 & $4,721.16$ \\
\hline & & 08-18-1999 & $4,722.4$ & 24.8 & 5.31 & $4,717.09$ \\
\hline & & 09-15-1999 & $4,722.4$ & 24.8 & 5.67 & $4,716.73$ \\
\hline & & $03-14-2000$ & $4,722.4$ & 24.8 & 4.66 & $4,717.74$ \\
\hline & & 04-26-2000 & $4,722.4$ & 24.8 & 3.96 & $4,718.44$ \\
\hline & & $05-24-2000$ & $4,722.4$ & 24.8 & 3.62 & $4,718.78$ \\
\hline & & $07-26-2000$ & $4,722.4$ & 24.8 & 4.81 & $4,717.59$ \\
\hline & & $08-30-2000$ & $4,722.4$ & 24.8 & 6.49 & $4,715.91$ \\
\hline & & $10-11-2000$ & $4,722.4$ & 24.8 & 5.95 & $4,716.45$ \\
\hline \multirow[t]{21}{*}{402117109204901} & \multirow[t]{21}{*}{ E2 } & 03-13-1996 & $4,722.6$ & 28.6 & 3.60 & $4,719.00$ \\
\hline & & 04-16-1996 & $4,722.6$ & 28.6 & 2.40 & $4,720.20$ \\
\hline & & 05-16-1996 & $4,722.6$ & 28.6 & 1.16 & $4,721.44$ \\
\hline & & 06-13-1996 & $4,722.6$ & 28.6 & 1.70 & $4,720.90$ \\
\hline & & 07-15-1996 & $4,722.6$ & 28.6 & 4.05 & $4,718.55$ \\
\hline & & 08-20-1996 & $4,722.6$ & 28.6 & 4.71 & $4,717.89$ \\
\hline & & 09-19-1996 & $4,722.6$ & 28.6 & 4.13 & $4,718.47$ \\
\hline & & 10-24-1996 & $4,722.6$ & 28.6 & 4.28 & $4,718.32$ \\
\hline & & 07-17-1997 & $4,722.6$ & 28.6 & 3.80 & $4,718.80$ \\
\hline & & 08-19-1997 & $4,722.6$ & 28.6 & 5.02 & $4,717.58$ \\
\hline & & 09-16-1997 & $4,722.6$ & 28.6 & 4.49 & $4,718.11$ \\
\hline & & 03-17-1999 & $4,722.6$ & 28.6 & 4.42 & $4,718.18$ \\
\hline & & 04-21-1999 & $4,722.6$ & 28.6 & 4.31 & $4,718.29$ \\
\hline & & 05-19-1999 & $4,722.6$ & 28.6 & 3.30 & $4,719.30$ \\
\hline & & 07-13-1999 & $4,722.6$ & 28.6 & 1.42 & $4,721.18$ \\
\hline & & 08-18-1999 & $4,722.6$ & 28.6 & 4.46 & $4,718.14$ \\
\hline & & 09-15-1999 & $4,722.6$ & 28.6 & 5.83 & $4,716.77$ \\
\hline & & 03-14-2000 & $4,722.6$ & 28.6 & 5.09 & $4,717.51$ \\
\hline & & 04-26-2000 & $4,722.6$ & 28.6 & 4.06 & $4,718.54$ \\
\hline & & $05-24-2000$ & $4,722.6$ & 28.6 & 2.93 & $4,719.67$ \\
\hline & & $07-26-2000$ & $4,722.6$ & 28.6 & 5.28 & $4,717.32$ \\
\hline
\end{tabular}


Table 11. Water levels for selected wells in the Stewart Lake Waterfowl Management Area near Jensen, Utah, water years 1996-2000—Continued

\begin{tabular}{|c|c|c|c|c|c|c|}
\hline Station number & Well & Date & $\begin{array}{l}\text { Land-surface } \\
\text { altitude } \\
\text { (feet) }\end{array}$ & $\begin{array}{c}\text { Well depth } \\
\text { below land } \\
\text { surface } \\
\text { (feet) }\end{array}$ & $\begin{array}{l}\text { Water level } \\
\text { below land } \\
\text { surface } \\
\text { (feet) }\end{array}$ & $\begin{array}{l}\text { Water-level } \\
\text { altitude } \\
\text { (feet) }\end{array}$ \\
\hline \multirow{2}{*}{$\begin{array}{l}402117109204901- \\
\text { Continued }\end{array}$} & \multirow[t]{2}{*}{ E2 } & $08-30-2000$ & $4,722.6$ & 28.6 & 6.58 & $4,716.02$ \\
\hline & & $10-11-2000$ & $4,722.6$ & 28.6 & 6.28 & $4,716.32$ \\
\hline \multirow[t]{24}{*}{402111109210701} & \multirow[t]{24}{*}{ E3 } & 03-13-1996 & $4,721.7$ & 27.8 & 3.00 & $4,718.70$ \\
\hline & & 04-16-1996 & $4,721.7$ & 27.8 & 2.10 & $4,719.60$ \\
\hline & & 05-17-1996 & $4,721.7$ & 27.8 & 0.56 & $4,721.14$ \\
\hline & & 06-13-1996 & $4,721.7$ & 27.8 & 1.20 & $4,720.50$ \\
\hline & & 07-16-1996 & $4,721.7$ & 27.8 & 2.76 & $4,718.94$ \\
\hline & & 08-20-1996 & $4,721.7$ & 27.8 & 3.75 & $4,717.95$ \\
\hline & & 09-19-1996 & $4,721.7$ & 27.8 & 3.53 & $4,718.17$ \\
\hline & & $10-24-1996$ & $4,721.7$ & 27.8 & 3.53 & $4,718.17$ \\
\hline & & 07-17-1997 & $4,721.7$ & 27.8 & 2.80 & $4,718.90$ \\
\hline & & 08-19-1997 & $4,721.7$ & 27.8 & 3.70 & $4,718.00$ \\
\hline & & 09-16-1997 & $4,721.7$ & 27.8 & 3.79 & $4,717.91$ \\
\hline & & 03-25-1998 & $4,721.7$ & 27.8 & 3.94 & $4,717.76$ \\
\hline & & 03-17-1999 & $4,721.7$ & 27.8 & 4.08 & $4,717.62$ \\
\hline & & 04-21-1999 & $4,721.7$ & 27.8 & 3.83 & $4,717.87$ \\
\hline & & 05-19-1999 & $4,721.7$ & 27.8 & 2.87 & $4,718.83$ \\
\hline & & 07-13-1999 & $4,721.7$ & 27.8 & .24 & $4,721.46$ \\
\hline & & 08-18-1999 & $4,721.7$ & 27.8 & 3.85 & $4,717.85$ \\
\hline & & 09-16-1999 & $4,721.7$ & 27.8 & 5.17 & $4,716.53$ \\
\hline & & $03-14-2000$ & $4,721.7$ & 27.8 & 4.65 & $4,717.05$ \\
\hline & & $04-26-2000$ & $4,721.7$ & 27.8 & 4.08 & $4,717.62$ \\
\hline & & $05-24-2000$ & $4,721.7$ & 27.8 & 3.27 & $4,718.43$ \\
\hline & & $07-26-2000$ & $4,721.7$ & 27.8 & 3.68 & $4,718.02$ \\
\hline & & $08-30-2000$ & $4,721.7$ & 27.8 & 5.97 & $4,715.73$ \\
\hline & & $10-11-2000$ & $4,721.7$ & 27.8 & 6.20 & $4,715.50$ \\
\hline \multirow[t]{20}{*}{402111109215901} & \multirow[t]{20}{*}{ W1 } & 03-13-1996 & $4,721.2$ & 27.7 & 1.30 & $4,719.90$ \\
\hline & & 04-16-1996 & $4,721.2$ & 27.7 & 1.52 & $4,719.68$ \\
\hline & & 05-16-1996 & $4,721.2$ & 27.7 & .28 & $4,720.92$ \\
\hline & & 06-13-1996 & $4,721.2$ & 27.7 & 1.05 & $4,720.15$ \\
\hline & & 07-16-1996 & $4,721.2$ & 27.7 & 2.27 & $4,718.93$ \\
\hline & & 08-20-1996 & $4,721.2$ & 27.7 & 2.59 & $4,718.61$ \\
\hline & & 09-19-1996 & $4,721.2$ & 27.7 & 2.25 & $4,718.95$ \\
\hline & & $10-24-1996$ & $4,721.2$ & 27.7 & 2.20 & $4,719.00$ \\
\hline & & 08-19-1997 & $4,721.2$ & 27.7 & .99 & $4,720.21$ \\
\hline & & 09-16-1997 & $4,721.2$ & 27.7 & .75 & $4,720.45$ \\
\hline & & 03-17-1999 & $4,721.2$ & 27.7 & 3.68 & $4,717.52$ \\
\hline & & 05-20-1999 & $4,721.2$ & 27.7 & 2.59 & $4,718.61$ \\
\hline & & 08-18-1999 & $4,721.2$ & 27.7 & 3.75 & $4,717.45$ \\
\hline & & 09-15-1999 & $4,721.2$ & 27.7 & 3.65 & $4,717.55$ \\
\hline & & $03-14-2000$ & $4,721.2$ & 27.7 & 3.95 & $4,717.25$ \\
\hline & & $04-27-2000$ & $4,721.2$ & 27.7 & 3.36 & $4,717.84$ \\
\hline & & $05-24-2000$ & $4,721.2$ & 27.7 & 2.89 & $4,718.31$ \\
\hline & & $07-26-2000$ & $4,721.2$ & 27.7 & 3.45 & $4,717.75$ \\
\hline & & $08-30-2000$ & $4,721.2$ & 27.7 & 5.57 & $4,715.63$ \\
\hline & & $10-11-2000$ & $4,721.2$ & 27.7 & 5.82 & $4,715.38$ \\
\hline
\end{tabular}


Table 11. Water levels for selected wells in the Stewart Lake Waterfowl Management Area near Jensen, Utah, water years 1996-2000—Continued

\begin{tabular}{|c|c|c|c|c|c|c|}
\hline Station number & Well & Date & $\begin{array}{l}\text { Land-surface } \\
\text { altitude } \\
\text { (feet) }\end{array}$ & $\begin{array}{l}\text { Well depth } \\
\text { below land } \\
\text { surface } \\
\text { (feet) }\end{array}$ & $\begin{array}{l}\text { Water level } \\
\text { below land } \\
\text { surface } \\
\text { (feet) }\end{array}$ & $\begin{array}{l}\text { Water-level } \\
\text { altitude } \\
\text { (feet) }\end{array}$ \\
\hline \multirow[t]{21}{*}{402106109220601} & \multirow[t]{21}{*}{ W2 } & $02-26-1996$ & $4,722.3$ & 30 & 2.20 & $4,720.10$ \\
\hline & & 04-16-1996 & $4,722.3$ & 30 & 2.16 & $4,720.14$ \\
\hline & & 05-16-1996 & $4,722.3$ & 30 & 1.05 & $4,721.25$ \\
\hline & & 06-13-1996 & $4,722.3$ & 30 & 1.94 & $4,720.36$ \\
\hline & & 07-16-1996 & $4,722.3$ & 30 & 3.10 & $4,719.20$ \\
\hline & & 08-20-1996 & $4,722.3$ & 30 & 3.47 & $4,718.83$ \\
\hline & & 09-19-1996 & $4,722.3$ & 30 & 3.06 & $4,719.24$ \\
\hline & & $10-24-1996$ & $4,722.3$ & 30 & 3.05 & $4,719.25$ \\
\hline & & 08-19-1997 & $4,722.3$ & 30 & .61 & $4,721.69$ \\
\hline & & 03-17-1999 & $4,722.3$ & 30 & 4.11 & $4,718.19$ \\
\hline & & 04-21-1999 & $4,722.3$ & 30 & 4.15 & $4,718.15$ \\
\hline & & 05-20-1999 & $4,722.3$ & 30 & 3.02 & $4,719.28$ \\
\hline & & 07-13-1999 & $4,722.3$ & 30 & .90 & $4,721.40$ \\
\hline & & 08-18-1999 & $4,722.3$ & 30 & 4.14 & $4,718.16$ \\
\hline & & 09-15-1999 & $4,722.3$ & 30 & 3.67 & $4,718.63$ \\
\hline & & 03-14-2000 & $4,722.3$ & 30 & 4.49 & $4,717.81$ \\
\hline & & 04-26-2000 & $4,722.3$ & 30 & 3.93 & $4,718.37$ \\
\hline & & $05-24-2000$ & $4,722.3$ & 30 & 3.60 & $4,718.70$ \\
\hline & & $07-26-2000$ & $4,722.3$ & 30 & 3.84 & $4,718.46$ \\
\hline & & 08-30-2000 & $4,722.3$ & 30 & 6.13 & $4,716.17$ \\
\hline & & $10-11-2000$ & $4,722.3$ & 30 & 6.36 & $4,715.94$ \\
\hline \multirow[t]{21}{*}{402056109220301} & \multirow[t]{21}{*}{ W3 } & 02-26-1996 & $4,721.4$ & 30 & 1.90 & $4,719.50$ \\
\hline & & 04-16-1996 & $4,721.4$ & 30 & 1.45 & $4,719.95$ \\
\hline & & 06-13-1996 & $4,721.4$ & 30 & 1.00 & $4,720.40$ \\
\hline & & 07-16-1996 & $4,721.4$ & 30 & 2.55 & $4,718.85$ \\
\hline & & 08-20-1996 & $4,721.4$ & 30 & 3.85 & $4,717.55$ \\
\hline & & 09-19-1996 & $4,721.4$ & 30 & 3.39 & $4,718.01$ \\
\hline & & $10-24-1996$ & $4,721.4$ & 30 & 3.14 & $4,718.26$ \\
\hline & & 07-17-1997 & $4,721.4$ & 30 & 1.73 & $4,719.67$ \\
\hline & & 09-16-1997 & $4,721.4$ & 30 & 1.22 & $4,720.18$ \\
\hline & & 03-17-1999 & $4,721.4$ & 30 & 3.25 & $4,718.15$ \\
\hline & & 04-21-1999 & $4,721.4$ & 30 & 3.52 & $4,717.88$ \\
\hline & & 05-20-1999 & $4,721.4$ & 30 & 1.85 & $4,719.55$ \\
\hline & & 07-13-1999 & $4,721.4$ & 30 & .21 & $4,721.19$ \\
\hline & & 08-18-1999 & $4,721.4$ & 30 & 3.40 & $4,718.00$ \\
\hline & & 09-15-1999 & $4,721.4$ & 30 & 3.61 & $4,717.79$ \\
\hline & & 03-14-2000 & $4,721.4$ & 30 & 3.67 & $4,717.73$ \\
\hline & & $04-26-2000$ & $4,721.4$ & 30 & 3.28 & $4,718.12$ \\
\hline & & $05-24-2000$ & $4,721.4$ & 30 & 2.98 & $4,718.42$ \\
\hline & & $06-26-2000$ & $4,721.4$ & 30 & 1.36 & $4,720.04$ \\
\hline & & $08-30-2000$ & $4,721.4$ & 30 & 5.47 & $4,715.93$ \\
\hline & & $10-11-2000$ & $4,721.4$ & 30 & 5.43 & $4,715.97$ \\
\hline
\end{tabular}


Table 12. Pedologic descriptions of borehole samples collected during installation of six monitoring wells in the Stewart Lake Waterfowl Management Area near Jensen, Utah, February 1996

[Data from Bureau of Reclamation]

\begin{tabular}{|c|c|c|c|c|c|}
\hline Well & $\begin{array}{l}\text { Depth below } \\
\text { land surface } \\
\text { (inches) }\end{array}$ & Soil description & Well & $\begin{array}{l}\text { Depth below } \\
\text { land surface } \\
\text { (inches) }\end{array}$ & Soil description \\
\hline \multirow[t]{11}{*}{ E1 } & $0-10$ & sandy loam & W1 & $0-5$ & silty clay loam \\
\hline & $10-13$ & silty clay & & $5-29$ & clay \\
\hline & $13-21$ & silty clay loam & & $29-42$ & sandy loam \\
\hline & $21-28$ & fine loamy sand & & $42-60$ & silty clay loam \\
\hline & $28-35$ & silty clay loam & & $60-65$ & fine sandy loam \\
\hline & $35-60$ & fine sandy loam & & $65-76$ & clay \\
\hline & $60-68$ & fine loamy sand & & $76-92$ & clay \\
\hline & $68-73$ & fine sandy clay loam & & $92-109$ & clay \\
\hline & $73-120$ & sandy loam & & $109-116$ & silty clay loam \\
\hline & $120-360$ & sandy loam, sand, and gravel & & $116-158$ & sand \\
\hline & & intermixed & & $158-240$ & sand, gravel, cobbles \\
\hline \multirow[t]{16}{*}{ E2 } & $0-6$ & loamy sand & & $240-360$ & sandy loam, sand, gravel, and \\
\hline & $6-24$ & loam & & & large cobbles \\
\hline & $24-32$ & loam & W2 & $0-25$ & silty clay loam \\
\hline & $32-38$ & loam & & $25-29$ & loamy sand \\
\hline & $38-43$ & fine sandy loam & & $29-43$ & silty clay \\
\hline & $43-49$ & sandy loam & & $43-53$ & silty clay \\
\hline & $49-58$ & silty clay loam & & $53-58$ & silty clay \\
\hline & $58-66$ & sandy loam & & $58-72$ & clay \\
\hline & $66-106$ & fine to medium sand & & $72-81$ & silty clay \\
\hline & $106-112$ & sandy loam & & $81-163$ & clay \\
\hline & $112-143$ & loamy sand & & $163-166$ & silty clay \\
\hline & $143-150$ & fine to medium sand and gravel & & $166-176$ & clay \\
\hline & $150-168$ & sand & & $176-180$ & sandy clay loam \\
\hline & $168-319$ & sandy loam, sand, gravel, and & & $180-360$ & sand and gravel \\
\hline & & cobbles & W3 & $0-24$ & silty clay loam \\
\hline & $319-343$ & shale & & $24-34$ & fine sandy loam \\
\hline \multirow[t]{10}{*}{ E3 } & $0-5$ & sandy loam & & $34-120$ & loamy sand \\
\hline & $5-8$ & silty clay loam & & $120-360$ & loamy sand, sand and gravel \\
\hline & $8-13$ & silty clay & & & intermixed \\
\hline & $13-18$ & silt loam & & & \\
\hline & $18-33$ & fine to medium sand & & & \\
\hline & $33-50$ & fine sandy loam & & & \\
\hline & $50-55$ & silty clay loam & & & \\
\hline & $55-60$ & $\begin{array}{l}\text { intermixed lenses of fine sand and } \\
\text { loamy sand }\end{array}$ & & & \\
\hline & $60-240$ & sand & & & \\
\hline & $240-360$ & sand, gravel, cobbles & & & \\
\hline
\end{tabular}


Table 13. Summary of discharge, physical properties, selenium concentration, and dissolved-solids concentration of water in Ashley Creek and selected tributaries near Vernal and Naples, Utah, water years 1991-2000

[Data from U.S. Geological Survey; $\mathrm{ft} \mathrm{t}^{3} / \mathrm{s}$, cubic feet per second; ${ }^{\circ} \mathrm{C}$, degrees Celsius; $\mu \mathrm{S} / \mathrm{cm}$, microsiemens per centimeter at $25^{\circ} \mathrm{C} ; \mu \mathrm{g} / \mathrm{L}$, micrograms per liter; $\mathrm{mg} / \mathrm{L}$, milligrams per liter; refer to fig. 3 and table 1 for site designation, in parentheses; <, less than; —, no data; >, greater than; E, estimated]

\begin{tabular}{|c|c|c|c|c|c|c|c|c|}
\hline Date & Time & $\begin{array}{l}\text { Discharge, } \\
\text { instant- } \\
\text { aneous } \\
\left(\mathrm{ft}^{3} / \mathrm{s}\right)\end{array}$ & $\begin{array}{c}\text { Temperature } \\
\left({ }^{\circ} \mathrm{C}\right)\end{array}$ & $\begin{array}{c}\text { Specific } \\
\text { conductance } \\
(\mu \mathrm{S} / \mathrm{cm})\end{array}$ & $\begin{array}{l}\text { pH, water, } \\
\text { whole, field } \\
\text { (standard } \\
\text { units) }\end{array}$ & $\begin{array}{l}\text { Selenium, } \\
\text { dissolved } \\
(\mu \mathrm{g} / \mathrm{L} \text { as } \mathrm{Se})\end{array}$ & $\begin{array}{l}\text { Selenium, } \\
\text { total } \\
(\mu \mathrm{g} / \mathrm{L} \text { as } \mathrm{Se})\end{array}$ & $\begin{array}{c}\text { Solids, } \\
\text { residue } \\
\text { at } 180^{\circ} \mathrm{C} \text {, } \\
\text { dissolved } \\
(\mathrm{mg} / \mathrm{L})\end{array}$ \\
\hline \multicolumn{9}{|c|}{ Ashley Creek at 500 North, near Steinaker Draw (AC 500) } \\
\hline $08-12-91$ & 1700 & 0.13 & 25.0 & 780 & 7.5 & $<1$ & - & 525 \\
\hline 04-23-92 & 1455 & 1.0 & 17.0 & 850 & 8.2 & $<1$ & - & - \\
\hline 08-03-92 & 2040 & .03 & 22.0 & 870 & 8.0 & 5.0 & - & - \\
\hline $03-25-93$ & 1130 & .10 & 3.5 & 886 & 8.2 & $<1$ & - & 540 \\
\hline 04-21-93 & 0900 & .37 & 6.0 & 1,020 & 7.9 & $<1$ & - & - \\
\hline $05-26-93$ & 0740 & $>200 \mathrm{E}$ & 6.5 & 96 & 8.3 & $<1$ & - & - \\
\hline $06-23-93$ & 1835 & 135 & 15.5 & 220 & 8.3 & $<1$ & - & - \\
\hline $07-28-93$ & 0715 & 11 & 17.0 & 312 & 8.1 & $<1$ & - & - \\
\hline $08-24-93$ & 1800 & 5.1 & 26.5 & 375 & 8.5 & $<1$ & - & - \\
\hline $10-13-93$ & 0800 & 2.0 & 10.5 & 610 & 8.1 & $<1$ & - & - \\
\hline $03-17-94$ & 0830 & 1.5 & 5.0 & 700 & 8.1 & $<1$ & - & - \\
\hline 04-13-94 & 0800 & .02 & 5.0 & 795 & 8.0 & $<1$ & - & - \\
\hline $05-18-94$ & 0800 & .51 & 11.0 & 430 & 8.0 & $<1$ & - & - \\
\hline $06-16-94$ & 0740 & .10 & 15.0 & 840 & 7.8 & $<1$ & - & - \\
\hline 07-14-94 & 0630 & .05 & 15.5 & 860 & 7.5 & $<1$ & - & - \\
\hline 08-16-94 & 1420 & .05 & 25.0 & 810 & 8.0 & $<1$ & - & - \\
\hline \multicolumn{9}{|c|}{ Unnamed tributary (East Bank), below Sewer Lagoon Spring (AT SL) } \\
\hline $10-23-91$ & 1015 & - & 12.0 & 3,400 & - & 240 & 240 & - \\
\hline \multicolumn{9}{|c|}{ Ashley Central Canal Return Flow, at Ashley Creek (ACC AC) } \\
\hline $08-13-91$ & 0900 & .09 & 16.5 & 1,730 & 7.7 & - & - & - \\
\hline \multicolumn{9}{|c|}{4930 tributary (West Bank) above Sadlier Draw (AT4930) } \\
\hline 08-13-91 & 1400 & .82 & 19.0 & 1,700 & 6.8 & 6.0 & - & 1,460 \\
\hline \multicolumn{9}{|c|}{ Sadlier Draw at mouth-Sunshine/Burns Bench runoff (SD mouth) } \\
\hline $08-13-91$ & 1610 & - & - & 7,000 & - & 1,300 & - & 6,580 \\
\hline $10-23-91$ & 1120 & - & 10.5 & 7,000 & - & 1,100 & 1,300 & - \\
\hline $06-22-95$ & 1205 & 26 & 20.0 & 3,780 & 8.1 & 13 & - & - \\
\hline \multicolumn{9}{|c|}{ Ashley Creek at 6550 East, near Naples, Utah (AC 6550) } \\
\hline $08-13-91$ & 1615 & - & 28.0 & 2,250 & - & 38 & - & - \\
\hline $05-18-93$ & 1600 & - & - & 108 & - & 6.0 & - & 752 \\
\hline $06-17-93$ & 1255 & - & - & 470 & - & 6.0 & - & 308 \\
\hline \multicolumn{9}{|c|}{ Unnamed tributary 14 (West Bank), north of Highway 40 (AT 14) } \\
\hline $08-13-91$ & 1300 & .09 & 21.5 & 5,250 & 7.4 & 22 & - & 5,250 \\
\hline \multicolumn{9}{|c|}{ Unnamed tributary 12 (East Bank), north of Highway 40 (AT 12) } \\
\hline 08-13-91 & 1205 & .02 & 27.0 & 3,420 & 8.0 & 7.0 & - & 3,050 \\
\hline \multicolumn{9}{|c|}{ Unnamed tributary 4 (East Bank), north of Highway 40 (AT 4) } \\
\hline $08-14-91$ & 1100 & .01 & 15.5 & 4,500 & 7.5 & 57 & - & 4,360 \\
\hline \multicolumn{9}{|c|}{ Unnamed tributary 3 (East Bank), north of Highway 40 (AT 3) } \\
\hline $08-14-91$ & 1030 & .05 & 18.0 & 2,720 & 7.8 & - & - & - \\
\hline
\end{tabular}


Table 13. Summary of discharge, physical properties, selenium concentration, and dissolved-solids concentration of water in Ashley Creek and selected tributaries near Vernal and Naples, Utah, water years 1991-2000—Continued

\begin{tabular}{|c|c|c|c|c|c|c|c|c|}
\hline Date & Time & $\begin{array}{c}\text { Discharge, } \\
\text { instant- } \\
\text { aneous } \\
\left(\mathrm{ft}^{3} / \mathrm{s}\right)\end{array}$ & $\begin{array}{c}\text { Temperature } \\
\left({ }^{\circ} \mathrm{C}\right)\end{array}$ & $\begin{array}{c}\text { Specific } \\
\text { conductance } \\
(\mu \mathrm{S} / \mathrm{cm})\end{array}$ & $\begin{array}{l}\mathrm{pH} \text {, water, } \\
\text { whole, field } \\
\text { (standard } \\
\text { units) }\end{array}$ & $\begin{array}{l}\text { Selenium, } \\
\text { dissolved } \\
(\mu \mathrm{g} / \mathrm{L} \text { as } \mathrm{Se})\end{array}$ & $\begin{array}{l}\text { Selenium, } \\
\text { total } \\
(\mu \mathrm{g} / \mathrm{L} \text { as } \mathrm{Se})\end{array}$ & $\begin{array}{c}\text { Solids, } \\
\text { residue } \\
\text { at } 180^{\circ} \mathrm{C} \text {, } \\
\text { dissolved } \\
(\mathrm{mg} / \mathrm{L})\end{array}$ \\
\hline \multicolumn{9}{|c|}{ Mantle Gulch at mouth, inflow to Ashley Creek (MG mouth) } \\
\hline $08-14-91$ & 1000 & .11 & 14.0 & 3,700 & 7.6 & 150 & - & 3,530 \\
\hline \multicolumn{9}{|c|}{ Ashley Creek near Jensen, Utah (AC 40) } \\
\hline $08-14-91$ & 0930 & 6.8 & 20.5 & 2,330 & 7.7 & 57 & - & 1,890 \\
\hline $10-23-91$ & 0915 & - & 10.5 & 2,100 & - & 44 & 55 & - \\
\hline
\end{tabular}

Ashley Creek below Union Canal Diversion near Jensen, Utah (AC 6750) (table 5)

Ashley Creek at mouth at the Green River (AC mouth)

$\begin{array}{lllllllll}07-30-91 & 1230 & - & 25.5 & 1,800 & - & 24 & -\end{array}$


Table 14. Discharge, physical properties, and chemical analyses of water from ponds, canals, and drains in the Ashley Creek drainage near Vernal and Naples, Utah, 1991-93

[Data from U.S. Geological Survey; $\mathrm{ft}^{3} / \mathrm{s}$, cubic feet per second; ${ }^{\circ} \mathrm{C}$, degrees Celsius; $\mu \mathrm{S} / \mathrm{cm}$, microsiemens per centimeter at $25^{\circ} \mathrm{C} ; \mathrm{mg} / \mathrm{L}$, milligrams per liter; $\mu \mathrm{g} / \mathrm{g}$, micrograms per gram; - , no data; <, less than; E, estimated value]

\begin{tabular}{|c|c|c|c|c|c|c|c|c|}
\hline Site designation & Date & Time & $\begin{array}{l}\text { Discharge, } \\
\text { instan- } \\
\text { taneous } \\
\left(\mathrm{ft}^{3} / \mathrm{s}\right)\end{array}$ & $\begin{array}{c}\text { Temper- } \\
\text { ature } \\
\left({ }^{\circ} \mathrm{C}\right)\end{array}$ & $\begin{array}{c}\text { Specific } \\
\text { conduc- } \\
\text { tance } \\
(\mu \mathrm{S} / \mathrm{cm})\end{array}$ & $\begin{array}{c}\mathrm{pH}, \\
\text { water, whole, } \\
\text { field } \\
\text { (standard } \\
\text { units) }\end{array}$ & $\begin{array}{l}\text { Selenium, } \\
\text { dissolved } \\
\text { (mg/L as } \\
\text { Se) }\end{array}$ & $\begin{array}{c}\text { Sele- } \\
\text { nium, } \\
\text { total in } \\
\text { bottom } \\
\text { material } \\
(\mu \mathrm{g} / \mathrm{g})\end{array}$ \\
\hline Rock Point Canal at 1500 West in Vernal & $06-18-92$ & 0820 & - & 10.0 & 350 & 8.1 & $<1$ & - \\
\hline 17A2 Drain near Vernal & 07-24-91 & 1620 & .09 & 12.0 & 920 & - & - & - \\
\hline 17B1 Drain, Vernal & $07-25-91$ & 0915 & .10 & 13.0 & 685 & - & $<1$ & - \\
\hline 17B2 Drain, Vernal & $07-25-91$ & 0920 & .10 & 12.5 & 800 & - & 2.0 & - \\
\hline 17A1 Drain, Vernal & $07-25-91$ & 0910 & .20 & 11.0 & 780 & - & 3.0 & - \\
\hline 16A1 Drain, Vernal & $07-25-91$ & 0845 & .04 & 10.0 & 810 & - & 2.0 & - \\
\hline Steinaker Service Canal at 500 North in Vernal & $06-18-92$ & 0800 & - & 11.0 & 290 & 7.7 & $<1$ & - \\
\hline 20A2 Drain, Vernal & $07-25-91$ & 0950 & .05 & 18.0 & 640 & - & $<1$ & - \\
\hline Amos/Merkley Drain in Maeser & $07-24-91$ & 1610 & 2.2 & 13.0 & 920 & - & 3.0 & - \\
\hline 22A1 Drain near Vernal & $07-24-91$ & 1555 & 2.4 & 14.0 & 860 & - & 2.0 & - \\
\hline 27A2 Drain, Vernal & $07-25-91$ & 0815 & 2.1 & 13.0 & 1,440 & - & 2.0 & - \\
\hline 27C2 Drain, Vernal & $07-24-91$ & 1530 & .54 & - & 1,240 & - & 2.0 & - \\
\hline 26A2 Drain, Vernal & $07-24-91$ & 1515 & .64 & 12.0 & 1,550 & - & 3.0 & - \\
\hline 26B2 Drain near Vernal & 07-24-91 & 1445 & .46 & - & 1,300 & - & 2.0 & - \\
\hline \multirow[t]{2}{*}{ 30B1 Drain near Naples } & $07-24-91$ & 1015 & .21 & 16.0 & 1,260 & - & 4.0 & - \\
\hline & $06-18-92$ & 1315 & 1.4 & 10.5 & 1,310 & 7.2 & 6.0 & $<1$ \\
\hline 26C1 Drain, Vernal & $07-24-91$ & 1430 & .05 & 13.0 & 1,260 & - & 1.0 & - \\
\hline 26C2 Drain near Vernal & $07-24-91$ & 1415 & .05 & 13.0 & 1,160 & - & 3.0 & - \\
\hline 25A2 Drain, Vernal & 07-24-91 & 1345 & .60 & 12.0 & 1,280 & - & 2.0 & - \\
\hline Pond 30A near Naples & 08-10-93 & 1210 & - & - & 1,400 & - & $<1$ & 5 \\
\hline 29A1 Drain near Naples & $07-24-91$ & 1045 & .10 & 14.5 & 1,900 & - & 3.0 & - \\
\hline \multirow[t]{2}{*}{ Open Pilot Drain near Vernal } & $08-12-91$ & 1500 & .22 & 13.0 & 1,380 & 7.1 & 6.0 & - \\
\hline & $06-18-92$ & 0900 & .01 & 11.5 & 1,400 & 7.5 & 5.0 & 3 \\
\hline \multirow[t]{2}{*}{ Pilot Drain near Vernal } & $07-24-91$ & 1200 & .11 & 13.5 & 1,800 & - & 4.0 & - \\
\hline & $06-18-92$ & 1100 & $<.01$ & 12.0 & 1,750 & 7.2 & 5.0 & 2 \\
\hline Pond 31A1 at 1830 E., 1700 South in Naples & $08-10-93$ & 1300 & - & - & 3,200 & - & $<1$ & 1 \\
\hline \multirow[t]{2}{*}{ 31A1 Drain, Vernal } & $07-24-91$ & 1150 & .08 & 14.5 & 2,600 & - & 8.0 & - \\
\hline & $06-18-92$ & 1145 & .09 & 12.0 & 2,800 & 7.1 & 9.0 & 1 \\
\hline \multirow[t]{2}{*}{ 32A1 Drain near Naples } & $07-24-91$ & 1100 & .09 & 14.0 & 1,650 & - & 3.0 & - \\
\hline & $06-18-92$ & 1250 & $<.01$ & 12.0 & 1,600 & 7.3 & 4.0 & 2 \\
\hline Ashley Central Canal at flume 2500 South, Naples & $06-18-92$ & 1410 & 1.9 & 12.0 & 490 & 8.4 & $<1$ & - \\
\hline Steinaker Service Canal at 2500 South near 500 West & $06-18-92$ & 1730 & - & 11.0 & 285 & 8.5 & $<1$ & - \\
\hline \multirow[t]{3}{*}{ 5A1 Drain near Naples } & $07-24-91$ & 1130 & .07 & 13.5 & 2,600 & - & 4.0 & - \\
\hline & $06-18-92$ & 1355 & $<.01$ & 11.0 & 3,500 & 7.4 & 10 & 2 \\
\hline & 08-11-93 & 0950 & - & - & 1,060 & - & 3.0 & - \\
\hline Pond on Ashley Central Canal at 2500 South Street, Naples & 08-10-93 & 1430 & - & - & 1,060 & - & $<1$ & $<1$ \\
\hline Ashley Central Canal return flow at Ashley Creek & 08-13-91 & 0900 & .09 & 16.5 & 1,730 & 7.7 & - & - \\
\hline Steinaker Service Canal at 4500 South in Vernal & $06-18-92$ & 1620 & 22 & 11.0 & 290 & 8.6 & $<1$ & - \\
\hline River Irrigation Canal at diversion, 6800 South in Naples & $06-18-92$ & 1500 & 2.6 & 11.0 & 1,820 & 8.2 & 47 & - \\
\hline Jackson Farm Pond at 5000 South Street, near Naples & 08-10-93 & 1715 & - & - & 1,300 & - & $<1$ & 2 \\
\hline Mantle Gulch at mouth, inflow to Ashley Creek & $08-14-91$ & 1000 & .11 & 14.0 & 3,700 & 7.6 & 150 & - \\
\hline \multirow[t]{2}{*}{ Highline Canal near Asphalt Ridge near Vernal } & $08-13-91$ & 1600 & 2.1 & 23.0 & 265 & 7.5 & $<1$ & - \\
\hline & $06-18-92$ & 1655 & 7.9 & 11.0 & 280 & 8.6 & $<1$ & - \\
\hline \multirow[t]{2}{*}{ Squires Farm pond on Steinaker Service Canal } & 08-10-93 & 1100 & - & - & - & - & - & 2 \\
\hline & $08-11-93$ & 1100 & - & - & 1,550 & - & 6.0 & - \\
\hline Steinaker Service Canal at Mantle Gulch, near Vernal & 08-13-91 & 1700 & .09 & 20.0 & 2,350 & 7.1 & 12 & - \\
\hline Upper Mantle Gulch at US 45 road to Bonanza & $05-19-93$ & 1120 & .01 & - & 3,350 & - & $<1$ & - \\
\hline Mantle Gulch at Oilfield Road East, Naples & 05-19-93 & 1330 & .69 & 18.0 & 4,100 & - & 500 & - \\
\hline
\end{tabular}


Table 14. Discharge, physical properties, and chemical analyses of water from ponds, canals, and drains in the Ashley Creek drainage near Vernal and Naples, Utah, 1991-93-Continued

\begin{tabular}{|c|c|c|c|c|c|c|c|c|c|c|c|c|c|}
\hline $\begin{array}{c}\text { Hardness, } \\
\text { total } \\
(\mathrm{mg} / \mathrm{L} \text { as } \\
\left.\mathrm{CaCO}_{3}\right)\end{array}$ & $\begin{array}{l}\text { Calcium, } \\
\text { dissolved } \\
\text { (mg/L as } \\
\text { Ca) }\end{array}$ & $\begin{array}{l}\text { Magne- } \\
\text { sium, } \\
\text { dis- } \\
\text { solved } \\
(\mathrm{mg} / \mathrm{L} \text { as } \\
\mathrm{Mg})\end{array}$ & $\begin{array}{c}\text { Sodium, } \\
\text { dissolved } \\
(\mathrm{mg} / \mathrm{L} \\
\text { as } \mathrm{Na})\end{array}$ & $\begin{array}{l}\text { Potassium, } \\
\text { dissolved } \\
\text { (mg/L as } \\
\mathrm{K} \text { ) }\end{array}$ & $\begin{array}{l}\text { Chloride, } \\
\text { dissolved } \\
\text { (mg/L as } \\
\text { Cl) }\end{array}$ & $\begin{array}{c}\text { Sulfate, } \\
\text { dis- } \\
\text { solved } \\
\left(\mathrm{mg}^{2} \mathrm{~L} \text { as }\right. \\
\left.\mathrm{SO}_{4}\right)\end{array}$ & $\begin{array}{l}\text { Fluoride, } \\
\text { dis- } \\
\text { solved } \\
\text { (mg/L as F) }\end{array}$ & $\begin{array}{l}\text { Silica, } \\
\text { dissolved } \\
(\mathrm{mg} / \mathrm{L} \text { as } \\
\left.\mathrm{SiO}_{2}\right)\end{array}$ & $\begin{array}{l}\text { Solids, } \\
\text { residue at } \\
180^{\circ} \mathrm{C}, \\
\text { dissolved } \\
\text { (mg/L) }\end{array}$ & $\begin{array}{l}\text { Solids, sum } \\
\text { of consti- } \\
\text { tuents, } \\
\text { dissolved } \\
\text { (mg/L) }\end{array}$ & $\begin{array}{l}\text { Solids, } \\
\text { dissolved } \\
\text { (tons per } \\
\text { day) }\end{array}$ & $\begin{array}{l}\text { Solids, } \\
\text { dissolved } \\
\text { (tons per } \\
\text { acre-foot) }\end{array}$ & $\begin{array}{c}\text { Alkalinity, } \\
\text { lab } \\
(\mathrm{mg} / \mathrm{L} \text { as } \\
\left.\mathrm{CaCO}_{3}\right)\end{array}$ \\
\hline- & - & - & - & - & - & - & - & - & - & - & - & - & - \\
\hline- & - & - & - & - & - & - & - & - & - & - & - & - & - \\
\hline- & - & - & - & - & - & - & - & - & 381 & - & - & - & - \\
\hline 410 & 88 & 45 & 15 & 1.1 & 8.3 & 110 & .90 & 36 & 507 & 500 & .14 & .69 & 327 \\
\hline 390 & 84 & 43 & 10 & .70 & 7.0 & 89 & .80 & 30 & 486 & 457 & .26 & .66 & 321 \\
\hline 430 & 90 & 49 & 15 & .50 & 7.3 & 110 & 1.3 & 33 & 516 & 506 & .06 & .70 & 334 \\
\hline- & - & - & - & - & - & - & - & - & - & - & - & - & - \\
\hline- & - & - & - & - & - & - & - & - & 394 & - & - & - & - \\
\hline 480 & 110 & 51 & 16 & .80 & 6.6 & 180 & .80 & 28 & 580 & 596 & 3.43 & .79 & 339 \\
\hline 460 & 110 & 45 & 15 & 2.2 & 7.5 & 170 & .70 & 22 & 550 & 562 & 3.52 & .75 & 316 \\
\hline 800 & 220 & 61 & 15 & 1.1 & 7.0 & 580 & .70 & 21 & 1,120 & 1,070 & 6.41 & 1.52 & 272 \\
\hline- & - & - & - & - & - & - & - & - & 908 & - & - & - & - \\
\hline 920 & 240 & 78 & 17 & 1.6 & 10 & 410 & 1.2 & 23 & 916 & 882 & .52 & 1.25 & 326 \\
\hline 700 & 170 & 66 & 18 & 1.1 & - & - & - & - & 902 & - & - & - & - \\
\hline 660 & 150 & 69 & 21 & 2.4 & 8.0 & 440 & .80 & 19 & 894 & 897 & 1.45 & 1.22 & 296 \\
\hline- & - & - & - & - & - & - & - & - & 790 & - & - & - & - \\
\hline- & - & - & - & - & - & - & - & - & - & - & - & - & - \\
\hline- & - & - & - & - & - & - & - & - & - & - & - & - & - \\
\hline 690 & 160 & 71 & 19 & 1.4 & 11 & 830 & 1.5 & 30 & 1,610 & 1,490 & .43 & 2.19 & 347 \\
\hline- & - & - & - & - & - & - & - & - & - & - & - & - & - \\
\hline 1,100 & 290 & 93 & 28 & 3.1 & - & - & - & - & 1,140 & - & - & - & - \\
\hline- & - & - & - & - & 12 & 790 & .90 & 18 & 1,420 & 1,390 & .42 & 1.93 & 310 \\
\hline- & - & - & - & - & - & - & - & - & - & - & - & - & - \\
\hline 1,000 & 240 & 100 & 43 & 2.5 & - & - & - & - & - & - & - & - & - \\
\hline- & - & - & - & - & - & - & - & - & - & - & - & - & - \\
\hline- & - & - & - & - & - & - & - & - & - & - & - & - & - \\
\hline 1,500 & 340 & 150 & 84 & 4.9 & - & - & - & - & - & - & - & - & - \\
\hline- & - & - & - & - & 22 & 1,500 & .80 & 23 & 2,380 & 2,240 & .51 & 3.24 & 200 \\
\hline 950 & 230 & 90 & 25 & 3.7 & - & - & - & - & - & - & - & - & - \\
\hline- & - & - & - & - & 9.5 & 610 & 1.2 & 31 & 1,270 & 1,240 & $.31 \mathrm{E}$ & $1.73 \mathrm{E}$ & 400 \\
\hline- & - & - & - & - & - & - & - & - & - & - & - & - & - \\
\hline- & - & - & - & - & - & - & - & - & - & - & - & - & - \\
\hline 1,700 & 500 & 100 & 32 & .80 & - & - & - & - & - & - & - & - & - \\
\hline- & - & - & - & - & 5.7 & 1,700 & 2.0 & 58 & 2,620 & 2,590 & $.50 \mathrm{E}$ & $3.56 \mathrm{E}$ & 321 \\
\hline- & - & - & - & - & - & - & - & - & - & - & - & - & - \\
\hline- & - & - & - & - & - & - & - & - & - & - & - & - & - \\
\hline- & - & - & - & - & - & - & - & - & - & - & - & - & - \\
\hline- & - & - & - & - & - & - & - & - & - & - & - & - & - \\
\hline- & - & - & - & - & - & - & - & - & - & - & - & - & - \\
\hline- & - & - & - & - & - & - & - & - & - & - & - & - & - \\
\hline- & - & - & - & - & - & - & - & - & 3,530 & - & - & - & - \\
\hline- & - & - & - & - & - & - & - & - & 158 & - & - & - & - \\
\hline- & - & - & - & - & - & - & - & - & - & - & - & - & - \\
\hline- & - & - & - & - & - & - & - & - & - & - & - & - & - \\
\hline- & - & - & - & - & - & - & - & - & - & - & - & - & - \\
\hline- & - & - & - & - & - & - & - & - & 2,240 & - & - & - & - \\
\hline- & - & - & - & - & - & - & - & - & - & - & - & - & - \\
\hline- & - & - & - & - & - & - & - & - & - & - & - & - & - \\
\hline
\end{tabular}


Table 15. Discharge, physical properties, and chemical and isotopic analyses of water from lagoons and seeps in the vicinity of the Vernal Sewage Lagoons and Winter Storage Pond near Naples, Utah, 1991-95

[Data from the U.S. Geological Survey; $\mathrm{ft}^{3} / \mathrm{s}$, cubic feet per second; ${ }^{\circ} \mathrm{C}$, degrees Celsius; $\mu \mathrm{S} / \mathrm{cm}$, microsiemens per centimeter at $25^{\circ} \mathrm{C} ; \mu \mathrm{g} / \mathrm{L}$, micrograms per liter; $\mathrm{mg} / \mathrm{L}$, milligrams per liter; permil, parts per thousand; —, no data; <, less than]

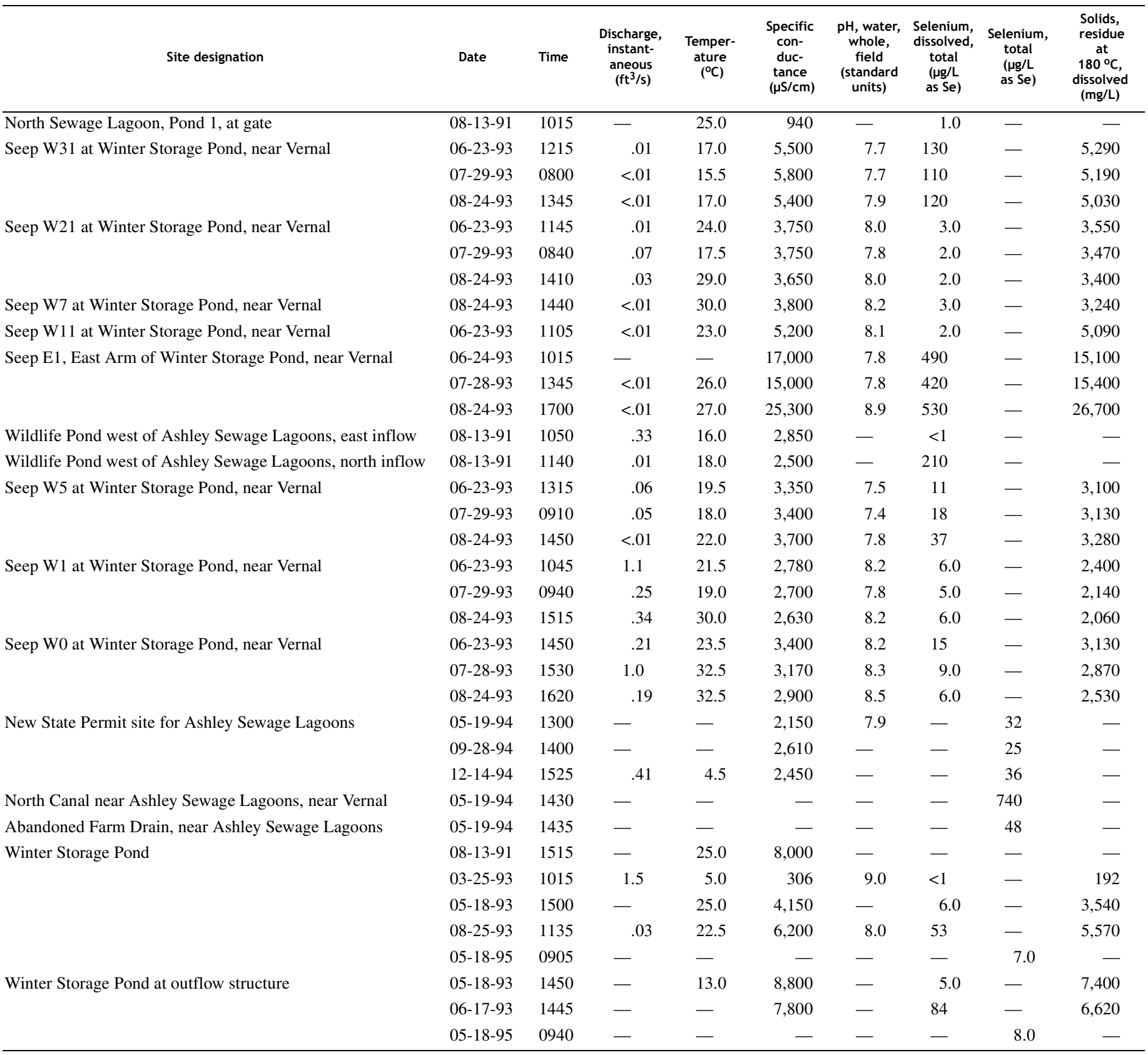


Table 15. Discharge, physical properties, and chemical and isotopic analyses of water from lagoons and seeps in the vicinity of the Vernal Sewage Lagoons and Winter Storage Pond near Naples, Utah, 1991-95—Continued

\begin{tabular}{|c|c|c|c|c|c|c|c|c|c|c|c|c|c|c|}
\hline $\begin{array}{c}\text { Solids, } \\
\text { sum of } \\
\text { constitu- } \\
\text { ents, } \\
\text { dissolved } \\
\text { (mg/L) }\end{array}$ & $\begin{array}{l}\text { Solids, } \\
\text { dissolved } \\
\text { (tons per } \\
\text { acre-foot) }\end{array}$ & $\begin{array}{l}\text { Hard- } \\
\text { ness, } \\
\text { total } \\
\left(\mathrm{mg}^{\prime} \mathrm{L} \text { as }\right. \\
\left.\mathrm{CaCO}_{3}\right)\end{array}$ & $\begin{array}{l}\text { Calcium } \\
\text { (mg/L as } \\
\text { Ca) }\end{array}$ & $\begin{array}{l}\text { Magne- } \\
\text { sium } \\
(\mathrm{mg} / \mathrm{L} \text { as } \\
\mathrm{Mg})\end{array}$ & $\begin{array}{l}\text { Sodium } \\
(\mathrm{mg} / \mathrm{L} \text { as } \\
\mathrm{Na})\end{array}$ & $\begin{array}{l}\text { Potas- } \\
\text { sium } \\
(\mathrm{mg} / \mathrm{L} \text { as } \\
\mathrm{K})\end{array}$ & $\begin{array}{l}\text { Chlo- } \\
\text { ride } \\
(\mathrm{mg} / \mathrm{L} \text { as } \\
\mathrm{Cl})\end{array}$ & $\begin{array}{l}\text { Sulfate } \\
(\mathrm{mg} / \mathrm{L} \text { as } \\
\left.\mathrm{SO}_{4}\right)\end{array}$ & $\begin{array}{l}\text { Flou- } \\
\text { ride } \\
\text { (mg/L as F) }\end{array}$ & $\begin{array}{c}\text { Silica } \\
(\mathrm{mg} / \mathrm{L} \text { as } \\
\left.\mathrm{SiO}_{2}\right)\end{array}$ & $\begin{array}{l}\text { Boron } \\
(\mu g / L \\
\text { as B) }\end{array}$ & $\begin{array}{c}\mathrm{H}-2 / \\
\mathrm{H}-1 \\
\text { stable } \\
\text { isotope ratio } \\
\text { of hydrogen } \\
\text { (permil) }\end{array}$ & $\begin{array}{c}\text { 0-18/ } \\
\text { O-16 stable } \\
\text { isotope } \\
\text { ratio of } \\
\text { oxygen } \\
\text { (permil) }\end{array}$ & $\begin{array}{c}\text { Alka-linity, } \\
\text { labor- } \\
\text { atory } \\
(\mathrm{mg} / \mathrm{L} \text { as } \\
\left.\mathrm{CaCO}_{3}\right)\end{array}$ \\
\hline 631 & 0.86 & 430 & 110 & 37 & 44 & 6.1 & 34 & 230 & 0.50 & 17 & - & -110.0 & -14.05 & 255 \\
\hline- & - & - & - & - & - & - & - & - & - & - & 2,200 & - & - & - \\
\hline- & - & - & - & - & - & - & - & - & - & - & 2,400 & - & - & - \\
\hline- & - & - & - & - & - & - & - & - & - & - & 2,300 & - & - & - \\
\hline- & - & - & - & - & - & - & - & - & - & - & 2,000 & - & - & - \\
\hline- & - & - & - & - & - & - & - & - & - & - & 1,900 & - & - & - \\
\hline- & - & - & - & - & - & - & - & - & - & - & 2,000 & - & - & - \\
\hline- & - & - & - & - & - & - & - & - & - & - & 2,600 & - & - & - \\
\hline- & - & - & - & - & - & - & - & - & - & - & 3,200 & - & - & - \\
\hline- & - & - & - & - & - & - & - & - & - & - & 2,600 & - & - & - \\
\hline- & - & - & - & - & - & - & - & - & - & - & 2,700 & - & - & - \\
\hline- & - & - & - & - & - & - & - & - & - & - & 2,800 & - & - & - \\
\hline 2,480 & 3.37 & 1,800 & 520 & 110 & 120 & 4.0 & 54 & 1,500 & 1.1 & 21 & - & -101.0 & -12.40 & 248 \\
\hline 1,930 & 2.63 & 1,300 & 350 & 93 & 150 & 4.6 & 64 & 1,100 & 1.0 & 22 & - & - & - & 250 \\
\hline- & - & - & - & - & - & - & - & - & - & - & 2,000 & - & - & - \\
\hline- & - & - & - & - & - & - & - & - & - & - & 2,000 & - & - & - \\
\hline- & - & - & - & - & - & - & - & - & - & - & 2,300 & - & - & - \\
\hline- & - & - & - & - & - & - & - & - & - & - & 1,500 & - & - & - \\
\hline- & - & - & - & - & - & - & - & - & - & - & 1,400 & - & - & - \\
\hline- & - & - & - & - & - & - & - & - & - & - & 1,500 & - & - & - \\
\hline- & - & - & - & - & - & - & - & - & - & - & 2,100 & - & - & - \\
\hline- & - & - & - & - & - & - & - & - & - & - & 2,100 & - & - & - \\
\hline- & - & - & - & - & - & - & - & - & - & - & 1,900 & - & - & - \\
\hline- & - & - & - & - & - & - & - & - & - & - & - & - & - & - \\
\hline- & - & - & - & - & - & - & - & - & - & - & - & - & - & - \\
\hline- & - & - & - & - & - & - & - & - & - & - & - & - & - & - \\
\hline- & - & - & - & - & - & - & - & - & - & - & - & - & - & - \\
\hline- & - & - & - & - & - & - & - & - & - & - & - & - & - & - \\
\hline 7,410 & 10.1 & 3,000 & 550 & 390 & 1,100 & 11 & 350 & 4,900 & 1.2 & 2.3 & - & -54.0 & -2.20 & 171 \\
\hline- & - & - & - & - & - & - & - & - & - & - & 60 & - & - & - \\
\hline- & - & - & - & - & - & - & - & - & - & - & 2,300 & - & - & - \\
\hline- & - & - & - & - & - & - & - & - & - & - & 3,100 & - & - & - \\
\hline- & - & - & - & - & - & - & - & - & - & - & - & - & - & - \\
\hline- & - & - & - & - & - & - & - & - & - & - & 4,900 & - & - & - \\
\hline- & - & - & - & - & - & - & - & - & - & - & 2,900 & - & - & - \\
\hline- & - & - & - & - & - & - & - & - & - & - & - & - & - & - \\
\hline
\end{tabular}

Table 16. Concentration of selected radiochemicals in bottom-sediment samples collected near irrigation drains J3 and J4 in the Stewart Lake Waterfowl Management Area near Jensen, Utah, 1992

[Data from the U.S. Geological Survey; pCi/g, picocuries per gram; $\mu \mathrm{g} / \mathrm{g}$, micrograms per gram; <, less than]

\begin{tabular}{|c|c|c|c|c|c|c|c|c|c|c|c|c|}
\hline $\begin{array}{l}\text { Drain } \\
\text { name }\end{array}$ & Date & Time & $\begin{array}{c}\text { Alpha as } \\
\text { Th-230 } \\
\text { (pCi/g) }\end{array}$ & $\begin{array}{l}\text { Alpha, } 2 \\
\text { sigma } \\
\text { Th-230 } \\
(\mathrm{pCi} / \mathrm{g})\end{array}$ & $\begin{array}{l}\text { Alpha, } \\
2 \text { sigma } \\
\text { as U } \\
\text { (ug/g) }\end{array}$ & $\begin{array}{c}\text { Beta, } \\
\text { Sr-90/Y90 } \\
\text { (pCi/L) }\end{array}$ & $\begin{array}{c}\text { Uranium } \\
-234 \\
(\mathrm{pCi} / \mathrm{g})\end{array}$ & $\begin{array}{c}\text { U-234 } \\
2 \text { Sigma } \\
(\mathrm{pCi} / \mathrm{g})\end{array}$ & $\begin{array}{l}\text { Uranium } \\
-235 \\
(\mathrm{pCi} / \mathrm{g})\end{array}$ & $\begin{array}{c}\text { U-235 } \\
2 \text { Sigma } \\
(\mathrm{pCi} / \mathrm{g})\end{array}$ & $\begin{array}{l}\text { Uranium } \\
-238 \\
(\mathrm{pCi} / \mathrm{g})\end{array}$ & $\begin{array}{c}\text { U-238 } \\
2 \text { Sigma } \\
(\mathrm{pCi} / \mathrm{g})\end{array}$ \\
\hline J3 & $07-15-92$ & 1010 & 20 & 9.1 & 12 & 25 & 11 & 1.1 & $<0$ & 0.07 & 8.1 & 0.8 \\
\hline $\mathrm{J} 4$ & $07-15-92$ & 0940 & 11 & 6.6 & 9.2 & 18 & 2.8 & .39 & $<0$ & .05 & 2.3 & .3 \\
\hline
\end{tabular}


Table 17. Concentration of selenium in bottom-sediment samples collected in and near Stewart Lake Waterfowl Management Area near Jensen, Utah, July 1994

[Data from the U.S. Geological Survey and Bureau of Reclamation; - , no data]

\begin{tabular}{|c|c|c|c|}
\hline \multirow{3}{*}{ Site ID number } & \multicolumn{3}{|c|}{ Depth below land surface (inches) } \\
\hline & $0-3$ & $3-6$ & $0-6$ \\
\hline & \multicolumn{3}{|c|}{$\begin{array}{c}\text { Selenium, total } \\
\text { (micrograms per gram, dry weight) }\end{array}$} \\
\hline $\mathrm{J} 1-0$ & 1 & 3 & - \\
\hline $\mathrm{J} 1-700$ & 11 & 11 & - \\
\hline $\mathrm{J} 1-900$ & 4 & 1 & - \\
\hline $\mathrm{J} 1-1200$ & 9 & 3 & - \\
\hline $\mathrm{J} 1-1700$ & 23 & 18 & - \\
\hline $\mathrm{J} 1-2000$ & 13 & 88 & - \\
\hline $\mathrm{J} 1-2200$ & 13 & 88 & - \\
\hline $\mathrm{J} 2$ Pond & 3 & 8 & - \\
\hline $\mathrm{J} 2-100$ & 2 & 2 & - \\
\hline $\mathrm{J} 4-10$ & - & - & 480 \\
\hline $\mathrm{J} 4-20$ & - & - & 340 \\
\hline $\mathrm{J} 4-60$ & 19 & 720 & - \\
\hline $\mathrm{J} 4-75$ & 360 & 44 & - \\
\hline J4 Pond 0 & - & - & 320 \\
\hline J4-100 Right & - & - & 160 \\
\hline J4-100 Left & - & - & 200 \\
\hline J4 Pond 200 & 150 & 73 & - \\
\hline $\mathrm{J} 4-200$ & 99 & 470 & - \\
\hline J4-400W & 360 & 20 & - \\
\hline $\mathrm{J} 4-400 \mathrm{E}$ & 160 & 28 & - \\
\hline J4-600W & 230 & 84 & - \\
\hline $\mathrm{J} 4-600 \mathrm{E}$ & 60 & 22 & - \\
\hline $\mathrm{J} 4-800 \mathrm{E}$ & 140 & 5 & - \\
\hline $\mathrm{J} 4-1600$ & 120 & 71 & - \\
\hline J4-1830-A & 130 & 28 & - \\
\hline J4-1830-B & 44 & 6 & - \\
\hline J4-1830-C & 55 & 3 & - \\
\hline $\mathrm{J} 4-2150-\mathrm{A}$ & 77 & 160 & - \\
\hline J4-2150-B & 85 & 23 & - \\
\hline
\end{tabular}

\begin{tabular}{|c|c|c|c|}
\hline \multirow{3}{*}{ Site ID number } & \multicolumn{3}{|c|}{ Depth below land surface (inches) } \\
\hline & $0-3$ & $3-6$ & $0-6$ \\
\hline & \multicolumn{3}{|c|}{$\begin{array}{c}\text { Selenium, total } \\
\text { (micrograms per gram, dry weight) }\end{array}$} \\
\hline $\mathrm{J} 4-2150-\mathrm{C}$ & 20 & 3 & - \\
\hline J4-2230-A & 59 & 6 & - \\
\hline J4-2230-B & 120 & 22 & - \\
\hline $\mathrm{J} 4-2230-\mathrm{C}$ & 22 & 3 & - \\
\hline $\mathrm{J} 4-2600$ & 160 & 27 & - \\
\hline $\mathrm{J} 4-3000$ & 49 & 2 & - \\
\hline J4-3200 Seep & 35 & 27 & - \\
\hline $\mathrm{J} 4-3200$ & 36 & 24 & - \\
\hline $\mathrm{J} 4-3500$ & 10 & 36 & - \\
\hline $\mathrm{J} 4-3800$ & 14 & 1 & - \\
\hline $\mathrm{J} 4-4600$ & 18 & 5 & - \\
\hline $\mathrm{J} 4-4200$ & 3 & 2 & - \\
\hline N1 E23 & 4 & 1 & - \\
\hline N2 E24 & 10 & 1 & - \\
\hline N5 E24 & 3 & 7 & - \\
\hline N6 E22 & 1 & 6 & - \\
\hline N13 E3 & 7 & 3 & - \\
\hline N13 E14 & 4 & 10 & - \\
\hline N13 E24 & 7 & 5 & - \\
\hline N13 E32 & 9 & 7 & - \\
\hline N20 E26 & 7 & 5 & - \\
\hline N26 E22 & 5 & 22 & - \\
\hline N27 E3 & 12 & 12 & - \\
\hline N27 E14 & 11 & 15 & - \\
\hline N27 E20 & 17 & 4 & - \\
\hline N28 E22 & 7 & 5 & - \\
\hline N32 E0 & 14 & 12 & - \\
\hline N32 E7 & 15 & 9 & - \\
\hline N35 E2 & 8 & 22 & - \\
\hline N38 E22 & 7 & 25 & - \\
\hline N39 E2 & 19 & 7 & - \\
\hline
\end{tabular}


Table 18. Concentration of selenium in soil and bottom-sediment samples collected from the east side of Stewart Lake Waterfowl

Management Area near Jensen, Utah, June 1995

[Data from Bureau of Reclamation; $\mu \mathrm{g} / \mathrm{g}$, micrograms per gram, dry weight; -, not determined]

\begin{tabular}{lcc}
\hline Site ID & $\begin{array}{c}\text { Depth of sample } \\
\text { below land surface } \\
\text { (inches) }\end{array}$ & $\begin{array}{c}\text { Total selenium } \\
(\boldsymbol{\mu g} / \mathrm{g})\end{array}$ \\
\hline B2 & $0-12$ & 0.4 \\
& $12-60$ & .3 \\
B3 & $0-18$ & .4 \\
& $18-60$ & .3 \\
B5 & $0-6$ & .3 \\
& $6-60$ & .3 \\
B6 & $0-12$ & 1.3 \\
B7 & $0-12$ & .5 \\
& $12-60$ & .4 \\
& $60-102$ & .2 \\
B8 & $0-24$ & .2 \\
& $24-60$ & .2 \\
\hline
\end{tabular}

Table 19. Concentration of selenium in bottom-sediment samples collected

near irrigation drains $\mathrm{J} 3$ and $\mathrm{J} 4$ in the Stewart Lake Waterfowl Management

Area near Jensen, Utah, June 1995

[Data from Bureau of Reclamation; - , no data]

\begin{tabular}{lcccccc}
\hline \multirow{5}{*}{$\begin{array}{c}\text { Site ID } \\
\text { number }\end{array}$} & \multicolumn{7}{c}{ Depth below land surface (inches) } \\
\cline { 2 - 7 } & $\mathbf{0 - 6}$ & $\mathbf{6 - 1 2}$ & $\mathbf{1 2 - 1 8}$ & $\mathbf{1 8 - 2 4}$ & $\mathbf{2 4 - 3 0}$ & $\mathbf{3 0 - 3 6}$ \\
\cline { 2 - 7 } Selenium, total & (micrograms & per gram, dry & weight) \\
\hline J3-30 & 30 & 16 & 4.1 & 2.7 & 2.9 & 2.9 \\
J3-60 & 46 & 21 & 2.3 & - & - & - \\
J3-90 & 14 & 2.2 & 2.4 & 2.4 & 2.4 & - \\
J3-150 & 30 & 2.7 & 1.8 & - & - & - \\
J3-210 & 68 & 10 & 1.7 & - & - & - \\
J3-270 & 4.3 & 1.2 & 3.5 & 8.8 & - & - \\
J3-330 & 23 & 7.1 & 4.8 & 9.3 & - & - \\
J3-390 & 3.5 & 1.8 & & - & - & - \\
J4-30 & 12 & 65 & 7 & 1.8 & - & - \\
J4-90 & 11 & .4 & & - & - & - \\
J4-150 & 10 & 3.3 & 3.9 & - & - & - \\
J4-210 & 9.4 & 2.9 & 1.8 & 2.2 & - & - \\
J4-270 & 9.4 & 4 & 1.7 & 1.5 & - & - \\
J4-330 & 1.9 & 6.4 & 2.4 & - & - & - \\
\hline
\end{tabular}


Table 20. Concentration of selenium in bottom-sediment samples collected from Stewart Lake Waterfowl Management Area near Jensen, Utah, August 1995

[Data from Bureau of Reclamation; - , no data]

\begin{tabular}{|c|c|c|c|c|c|c|c|}
\hline \multirow{3}{*}{$\begin{array}{l}\text { Site ID } \\
\text { number }\end{array}$} & \multirow{3}{*}{$\begin{array}{l}\text { Permanent } \\
\text { Site ID }\end{array}$} & \multicolumn{6}{|c|}{ Depth below land surface (inches) } \\
\hline & & $0-6$ & 6-12 & $12-18$ & 18-24 & $24-30$ & $30-36$ \\
\hline & & \multicolumn{6}{|c|}{ Selenium, total (micrograms per gram, dry weight) } \\
\hline $2048-2148$ & S1 & 14 & 8.2 & 10 & 12 & - & - \\
\hline $2049-2157$ & & 11 & 10 & 1.2 & 7 & - & - \\
\hline 2051-2131 & & 6.4 & 2.3 & .4 & - & - & - \\
\hline 2053-2143 & S2 & 8.8 & 22 & 3.2 & - & - & - \\
\hline $2055-2118$ & & 1.4 & .8 & .3 & - & - & - \\
\hline $2055-2154$ & S3 & 11 & 4 & 15 & - & - & - \\
\hline 2056-2144 & S4 & 13 & 12 & 2.4 & - & - & - \\
\hline 2057-2201 & S5 & 11 & .9 & - & - & - & - \\
\hline $2100-2127$ & S6 & 7.6 & 1.3 & 1.1 & - & - & - \\
\hline $2101-2218$ & & 1.2 & 2.1 & 3.1 & - & - & - \\
\hline $2102-2210$ & & 3.5 & 3.5 & 4 & - & - & - \\
\hline $2105-2122$ & S7 & 10 & 1.3 & .8 & - & - & - \\
\hline $2105-2145$ & S8 & 24 & 16 & 5.1 & - & - & - \\
\hline $2108-2225$ & & 2.7 & 2.5 & 4.4 & - & - & - \\
\hline $2110-2105$ & & 1 & .6 & .2 & .1 & - & - \\
\hline $2110-2130$ & S9 & 19 & 2.4 & .8 & .7 & - & - \\
\hline 2110-2202 & & 3.1 & 2.4 & - & - & - & - \\
\hline $2110-2202$ & & 3.3 & 5.1 & 2.8 & - & - & - \\
\hline $2112-2100$ & & 1.2 & .7 & - & - & - & - \\
\hline $2112-2158$ & & 11 & 22 & 5.5 & - & - & - \\
\hline $2112-2212$ & & 2.3 & .2 & & - & - & - \\
\hline $2113-2120$ & & 11 & 11 & 13 & 3.9 & - & - \\
\hline $2114-2211$ & & 3.5 & 2.6 & .5 & .4 & - & - \\
\hline $2116-2150$ & S11 & 26 & 4.5 & 1.7 & - & - & - \\
\hline $2117-2108$ & & 15 & 11 & 1 & - & - & - \\
\hline $2117-2132$ & S12 & 11 & 1.3 & .6 & - & - & - \\
\hline $2119-2158$ & & 2.6 & 1.7 & 1.6 & - & - & - \\
\hline $2120-2136$ & & 16 & 12 & .9 & - & - & - \\
\hline $2121-2120$ & & 27 & 11 & - & - & - & - \\
\hline $2121-2136$ & S14 & 21 & 3.3 & 2.8 & - & - & - \\
\hline $2123-2111$ & & 3.4 & 1.8 & 2.4 & - & - & - \\
\hline $2123-2130$ & & 18 & 5.4 & 1.6 & 3.2 & - & - \\
\hline $2124-2149$ & & 18 & 5.1 & 1.8 & - & - & - \\
\hline $2124-2203$ & & 2.1 & 1.1 & 1.5 & - & - & - \\
\hline $2125-2110$ & & 16 & 9.5 & 1.2 & - & - & - \\
\hline $2125-2113$ & & 22 & 3.4 & - & - & - & - \\
\hline $2126-2100$ & S17 & 11 & 1.7 & .5 & - & - & - \\
\hline $2126-2136$ & S18 & 18 & 12 & 4.7 & 19 & - & - \\
\hline $2127-2159$ & & 5 & 2.1 & - & - & - & - \\
\hline 2129-2059 & & 3.2 & 1.3 & .3 & 1.3 & - & - \\
\hline $2130-2146$ & & 21 & 3.7 & 7 & - & - & - \\
\hline $2131-2159$ & & 5.3 & 10 & - & - & - & - \\
\hline $2134-2158$ & & 20 & 36 & 40 & - & - & - \\
\hline $2135-2153$ & & .4 & .3 & 5.3 & 44 & 27 & 3.9 \\
\hline $2138-2043$ & & 4 & 8 & 10 & - & - & - \\
\hline
\end{tabular}


Table 21. Concentration of selenium species in bottom-sediment samples collected from Stewart Lake Waterfowl Management Area near Jensen, Utah, April 1996

[Data from Bureau of Reclamation; $\mu \mathrm{g} / \mathrm{g}$, micrograms per gram, dry weight]

\begin{tabular}{|c|c|c|c|c|c|c|c|}
\hline Site designation & $\begin{array}{l}\text { Depth of } \\
\text { sample below } \\
\text { land surface } \\
\text { (inches) }\end{array}$ & $\begin{array}{c}\text { Soluble } \\
\text { selenium } \\
(\mathrm{VI}) \\
(\mu \mathrm{g} / \mathrm{g})\end{array}$ & $\begin{array}{c}\text { Adsorbed } \\
\text { selenium } \\
(\mathrm{IV}) \\
(\mu \mathrm{g} / \mathrm{g})\end{array}$ & $\begin{array}{c}\text { Selenium } \\
(0) \\
(\mu \mathrm{g} / \mathrm{g})\end{array}$ & $\begin{array}{c}\text { Organic } \\
\text { selenium } \\
(\mu \mathrm{g} / \mathrm{g})\end{array}$ & $\begin{array}{c}\text { Selenium } \\
\text { oxides } \\
(\mu \mathrm{g} / \mathrm{g})\end{array}$ & $\begin{array}{l}\text { Selenium in } \\
\text { organic } \\
\text { material } \\
\text { separated } \\
\text { from } \\
\text { sediment } \\
(\mu \mathrm{g} / \mathrm{g})\end{array}$ \\
\hline \multirow[t]{10}{*}{ Drain Channel at east end at lake } & $0-0.39$ & 0.644 & 1.41 & 13.6 & 6.12 & 6.02 & 26.5 \\
\hline & $.39-.79$ & .727 & 1.31 & 13.9 & 10.3 & 5.65 & 30.4 \\
\hline & $.79-1.18$ & .647 & 1.24 & 14.0 & 12.2 & 5.50 & 31.2 \\
\hline & $1.18-1.97$ & 1.20 & 1.65 & 18.4 & 13.7 & 5.78 & 39.7 \\
\hline & $1.97-2.76$ & 1.40 & 2.05 & 18.2 & 11.0 & 6.60 & 40.4 \\
\hline & $2.76-3.94$ & 1.25 & 1.81 & 18.0 & 11.9 & 6.44 & 37.3 \\
\hline & $3.94-5.12$ & 1.06 & 1.61 & 13.3 & 15.8 & 5.66 & 37.2 \\
\hline & $5.12-6.30$ & .870 & 1.21 & 13.6 & 10.5 & 4.02 & 28.8 \\
\hline & $6.30-8.27$ & .207 & .380 & 6.12 & 6.73 & 1.54 & 15.5 \\
\hline & $8.27-10.2$ & .060 & .136 & .676 & 1.36 & .106 & 2.62 \\
\hline \multirow[t]{7}{*}{ Edge of lake at end of dike road } & 0-.39 & .111 & .260 & .692 & .836 & .080 & 2.14 \\
\hline & $.39-1.97$ & .081 & .151 & .771 & .914 & .069 & 2.13 \\
\hline & $.97-3.54$ & .138 & .251 & .520 & .683 & .064 & 1.75 \\
\hline & $3.54-4.72$ & .024 & .044 & .578 & .461 & .033 & 1.25 \\
\hline & $4.72-7.48$ & .047 & .108 & .620 & .544 & .031 & 1.43 \\
\hline & $7.48-10.2$ & .051 & .359 & .940 & 2.00 & .081 & 3.65 \\
\hline & $10.2-15.7$ & .062 & .181 & .163 & .089 & .017 & .59 \\
\hline $\mathrm{J} 3$ & $0-3.94$ & 1.15 & 1.65 & 36.8 & 23.0 & 8.78 & 70.4 \\
\hline $\mathrm{J} 4$ & $0-3.94$ & .101 & .132 & 1.93 & 1.98 & .125 & 3.8 \\
\hline S19 & $0-3.94$ & 2.366 & 2.45 & 48.5 & 62.9 & 5.91 & 124.0 \\
\hline West end of drain channel & $0-3.94$ & .540 & 1.15 & 12.2 & 10.3 & 5.13 & 27.9 \\
\hline Central reach of drain channel & $0-3.94$ & 1.47 & 1.91 & 12.9 & 14.5 & 3.95 & 34.5 \\
\hline West end of dike near end of road & $0-3.94$ & .095 & .221 & 4.41 & 3.68 & .272 & 8.6 \\
\hline Stewart Lake outlet (SLO) & $0-3.94$ & .172 & .334 & 4.36 & 4.41 & .440 & 9.6 \\
\hline
\end{tabular}


Table 22. Concentration of total selenium in suspended-sediment samples collected from Stewart Lake Waterfowl Management Area near Jensen, Utah, May 1997

[Data from Bureau of Reclamation; $\mu \mathrm{g} / \mathrm{g}$, micrograms per gram, dry weight]

\begin{tabular}{|c|c|c|c|c|}
\hline Site ID & Site description & Sample type & Date & $\begin{array}{l}\text { Total selenium } \\
\qquad(\mu \mathrm{g} / \mathrm{g})\end{array}$ \\
\hline SS1 & Center of first breach west of outlet bridge & Depth integrated & $05-20-97$ & 1.30 \\
\hline SS1 & Center of first breach west of outlet bridge & Grab sample & $05-20-97$ & .87 \\
\hline $\mathrm{SS} 2$ & Near west abutment south of outlet bridge & Depth integrated & $05-20-97$ & 1.10 \\
\hline $\mathrm{SS} 2$ & Near west abutment south of outlet bridge & Grab sample & $05-20-97$ & 1.00 \\
\hline SS3 & Center of second breach west of outlet bridge & Depth integrated & $05-20-97$ & .78 \\
\hline SS3 & Center of second breach west of outlet bridge & Grab sample & $05-20-97$ & .94 \\
\hline SS4 & Ashley Creek overflow at west boundary fence & Depth integrated & $05-20-97$ & .52 \\
\hline SS4 & Ashley Creek overflow at west boundary fence & Grab sample & $05-20-97$ & .51 \\
\hline SS5 & Center of inlet channel 100 feet downstream of Green River & Depth integrated & $05-21-97$ & .90 \\
\hline SS5 & Center of inlet channel 100 feet downstream of Green River & Grab sample & $05-21-97$ & .80 \\
\hline SS6 & Southwest of SS5 & Hand scoop (bed material) & $05-21-97$ & .14 \\
\hline SS6 & Southwest of SS5 & Hand scoop (bed material) & $05-21-97$ & .15 \\
\hline SS7 & Near west bank of Green River 120 feet upstream of inlet & Depth integrated & $05-21-97$ & .78 \\
\hline SS7 & Near west bank of Green River 120 feet upstream of inlet & Grab sample & $05-21-97$ & .81 \\
\hline
\end{tabular}

Table 23. Concentration of selenium in bottom-sediment samples collected from Green River backwaters near Stewart Lake Waterfowl Management Area near Jensen, Utah, July 1997

to July 1999

[Data from U.S. Fish and Wildlife Service; $\mu \mathrm{g} / \mathrm{g}$, micrograms per gram, dry weight; —, not determined]

\begin{tabular}{|c|c|c|c|c|}
\hline Site ID & Date & $\begin{array}{l}\text { Weight } \\
\text { (grams) }\end{array}$ & $\begin{array}{l}\text { Percent } \\
\text { moisture }\end{array}$ & $\begin{array}{l}\text { Selenium } \\
(\mu g / g)\end{array}$ \\
\hline \multirow[t]{4}{*}{$\overline{\text { GR1 }}$} & 07-29-1997 & 2,249 & 2.0 & $<0.40$ \\
\hline & 06-17-1998 & 774 & 36.0 & $<1.00$ \\
\hline & 07-14-1998 & 658 & 23.1 & $<1.00$ \\
\hline & 07-27-1999 & 574 & 31.6 & $<1.00$ \\
\hline \multirow[t]{2}{*}{ GR2 } & 07-29-1997 & 1,654 & - & $<.40$ \\
\hline & 07-14-1998 & 684 & 30.6 & $<1.00$ \\
\hline \multirow[t]{5}{*}{ GR3 } & 07-29-1997 & 1,594 & - & $<.40$ \\
\hline & 06-17-1998 & 769 & 37.3 & $<1.00$ \\
\hline & 07-14-1998 & 798 & 17.2 & $<1.00$ \\
\hline & 08-10-1998 & 844 & 36.2 & $<1.00$ \\
\hline & 07-21-1999 & 607 & 46.0 & $<1.00$ \\
\hline \multirow[t]{5}{*}{ GR4 } & 07-29-1997 & 673 & - & 1.8 \\
\hline & 06-17-1998 & 700 & 31.1 & 1.6 \\
\hline & 07-14-1998 & 589 & 26.2 & 1.4 \\
\hline & 07-27-1999 & 554 & 31.1 & $<1.00$ \\
\hline & 07-27-1999 & 407 & 31.0 & $<1.00$ \\
\hline \multirow[t]{2}{*}{ GR5 } & 07-29-1997 & 1325 & - & $<.40$ \\
\hline & 07-27-1999 & 547 & 27.4 & $<1.00$ \\
\hline \multirow[t]{3}{*}{ GR6 } & 06-17-1998 & 618 & 41.0 & 1.2 \\
\hline & 08-11-1998 & 876 & 25.8 & $<1.00$ \\
\hline & 07-27-1999 & 581 & 35.5 & $<1.00$ \\
\hline $\mathrm{J} 1 \mathrm{~B}$ & $06-28-2000$ & 257 & 41.2 & $<1.00$ \\
\hline
\end{tabular}


Table 24. Concentration of selenium in bottom-sediment samples collected from Stewart Lake Waterfowl Management Area near Jensen, Utah, July 1997-December 2000

[Data from Bureau of Reclamation; - , no data]

\begin{tabular}{|c|c|c|c|c|c|c|c|c|c|c|}
\hline \multirow{3}{*}{$\begin{array}{c}\text { Site ID } \\
\text { number }\end{array}$} & \multirow{3}{*}{$\begin{array}{l}\text { Permanent } \\
\text { site ID }\end{array}$} & \multirow{3}{*}{ Date } & \multicolumn{8}{|c|}{ Depth below land surface (inches) } \\
\hline & & & $0-3$ & $3-6$ & $6-12$ & $12-18$ & $18-24$ & $24-30$ & $30-36$ & $8-24$ \\
\hline & & & \multicolumn{8}{|c|}{ Selenium, total (micrograms per gram, dry weight) } \\
\hline $2048-2148$ & S1 & July 1997 & 13 & 7.1 & - & - & - & - & - & - \\
\hline $2048-2148$ & S1 & September 1998 & 17 & 23 & - & - & - & - & - & - \\
\hline $2048-2148$ & S1 & November 1999 & 16 & 26 & - & - & - & - & - & - \\
\hline $2048-2148$ & S1 & April 2000 & 9.9 & 10 & - & - & - & - & - & - \\
\hline $2048-2148$ & S1 & December 2000 & 12 & 21 & 16 & 5.2 & 2.6 & 2.6 & 2.1 & \\
\hline $2053-2143$ & S2 & July 1997 & 9.6 & 4.8 & - & - & - & - & - & - \\
\hline $2053-2143$ & S2 & September 1998 & 8.7 & 12.0 & - & - & - & - & - & - \\
\hline $2053-2143$ & S2 & November 1999 & 18 & 7.6 & - & - & - & - & - & - \\
\hline $2053-2143$ & S2 & December 2000 & 7.8 & 6.2 & - & - & - & - & - & - \\
\hline $2055-2154$ & S3 & July 1997 & 13 & 13 & - & - & - & - & - & - \\
\hline $2055-2154$ & S3 & September 1998 & 13.0 & 14.0 & - & - & - & - & - & - \\
\hline $2055-2154$ & S3 & November 1999 & 13 & 13 & - & - & - & - & - & - \\
\hline $2055-2154$ & S3 & December 2000 & 9.1 & 10 & - & - & - & - & - & - \\
\hline $2056-2144$ & S4 & July 1997 & 6.5 & 9.4 & - & - & - & - & - & - \\
\hline $2056-2144$ & S4 & September 1998 & 14.0 & 14.0 & - & - & - & - & - & - \\
\hline $2056-2144$ & S4 & November 1999 & 10 & 9 & - & - & - & - & - & - \\
\hline $2056-2144$ & S4 & December 2000 & 7.5 & 11 & - & - & - & - & - & - \\
\hline $2057-2201$ & S5 & July 1997 & 5.1 & 5.4 & - & - & - & - & - & - \\
\hline $2057-2201$ & S5 & September 1998 & 6.9 & 7.1 & - & - & - & - & - & - \\
\hline $2057-2201$ & S5 & November 1999 & 12 & 8.6 & - & - & - & - & - & - \\
\hline $2057-2201$ & S5 & December 2000 & 7.2 & 8 & 6.7 & 2.8 & 1.7 & .8 & .6 & \\
\hline $2100-2127$ & S6 & July 1997 & 14 & 10 & - & - & - & - & - & - \\
\hline $2100-2127$ & S6 & September 1998 & 18.0 & 6.0 & - & - & - & - & - & - \\
\hline $2100-2127$ & S6 & November 1999 & 14 & 7.1 & - & - & - & - & - & - \\
\hline $2100-2127$ & S6 & December 2000 & 12 & 5.2 & - & - & - & - & - & - \\
\hline $2105-2122$ & S7 & July 1997 & 8.1 & 8.2 & - & - & - & - & - & - \\
\hline $2105-2122$ & S7 & September 1998 & 11.0 & 20.0 & - & - & - & - & - & - \\
\hline $2105-2122$ & S7 & November 1999 & 16 & 17 & - & - & - & - & - & - \\
\hline $2105-2122$ & S7 & December 2000 & 13 & 14 & - & - & - & - & - & - \\
\hline $2105-2145$ & S8 & July 1997 & 10 & 4.9 & - & - & - & - & - & - \\
\hline $2105-2145$ & S8 & September 1998 & 8.4 & 6.3 & - & - & - & - & - & - \\
\hline $2105-2145$ & S8 & November 1999 & 7.7 & 9.8 & - & - & - & - & - & - \\
\hline $2105-2145$ & S8 & December 2000 & 7 & 9.8 & - & - & - & - & - & - \\
\hline $2110-2130$ & S9 & July 1997 & 12 & 20 & - & - & - & - & - & - \\
\hline $2110-2130$ & S9 & September 1998 & 13.0 & 21.0 & - & - & - & - & - & - \\
\hline $2110-2130$ & S9 & November 1999 & 19 & 32 & - & - & - & - & - & - \\
\hline $2110-2130$ & S9 & November 1999 & 19 & & - & - & - & - & - & - \\
\hline 2113-2119 & $\mathrm{S} 10$ & September 1998 & 8.6 & 11.0 & - & - & - & - & - & - \\
\hline $2113-2119$ & S10 & November 1999 & 10 & 23 & - & - & - & - & - & - \\
\hline 2113-2119 & $\mathrm{S} 10$ & December 2000 & 6.2 & 14 & 13 & 5.1 & 1.4 & .64 & .56 & \\
\hline $2116-2150$ & S11 & July 1997 & 14 & 5.1 & - & - & - & - & - & - \\
\hline $2116-2150$ & S11 & September 1998 & 8.0 & 12.0 & - & - & - & - & - & - \\
\hline $2116-2150$ & S11 & November 1999 & 6.8 & 16 & - & - & - & - & - & - \\
\hline $2116-2150$ & S11 & December 2000 & 8.6 & 11 & - & - & - & - & - & - \\
\hline $2117-2132$ & $\mathrm{~S} 12$ & July 1997 & 7.1 & 27 & - & - & - & - & - & - \\
\hline $2117-2132$ & $\mathrm{~S} 12$ & September 1998 & 11.0 & 20.0 & - & - & - & - & - & - \\
\hline
\end{tabular}


Table 24. Concentration of selenium in bottom-sediment samples collected from Stewart Lake Waterfowl Management Area near Jensen, Utah, July 1997-December 2000-Continued

\begin{tabular}{|c|c|c|c|c|c|c|c|c|c|c|}
\hline \multirow{3}{*}{$\begin{array}{c}\text { Site ID } \\
\text { number }\end{array}$} & \multirow{3}{*}{$\begin{array}{l}\text { Permanent } \\
\text { site ID }\end{array}$} & \multirow{3}{*}{ Date } & \multicolumn{8}{|c|}{ Depth below land surface (inches) } \\
\hline & & & $0-3$ & $3-6$ & $6-12$ & $12-18$ & $18-24$ & $24-30$ & $30-36$ & $8-24$ \\
\hline & & & \multicolumn{8}{|c|}{ Selenium, total (micrograms per gram, dry weight) } \\
\hline $2117-2132$ & S12 & November 1999 & 15 & 31 & - & - & - & - & - & - \\
\hline $2117-2132$ & S12 & December 2000 & 10 & 23 & - & - & - & - & - & - \\
\hline $2118-2105$ & S13 & September 1998 & 6.5 & 16.0 & - & - & - & - & - & - \\
\hline $2118-2105$ & S13 & November 1999 & 5.7 & 15 & - & - & - & - & - & 6.2 \\
\hline $2118-2105$ & $\mathrm{~S} 13$ & December 2000 & 5.4 & 13 & - & - & - & - & - & - \\
\hline $2121-2136$ & S14 & July 1997 & 15 & 7.5 & - & - & - & - & - & - \\
\hline $2121-2136$ & S14 & September 1998 & 15.0 & 37.0 & - & - & - & - & - & - \\
\hline $2121-2136$ & S14 & November 1999 & 19 & 20 & - & - & - & - & - & 9.8 \\
\hline $2121-2136$ & S14 & December 2000 & 15 & 26 & - & - & - & - & - & - \\
\hline $2123-2144$ & S15 & September 1998 & 29.0 & 32.0 & - & - & - & - & - & - \\
\hline $2123-2144$ & S15 & November 1999 & 28 & 22 & - & - & - & - & - & - \\
\hline $2123-2144$ & S15 & December 2000 & 31 & 26 & 7.4 & 5.9 & 5.6 & 9 & 3.9 & - \\
\hline $2125-2112$ & S16 & September 1998 & 39.0 & 23.0 & - & - & - & - & - & - \\
\hline $2125-2112$ & S16 & November 1999 & 26 & 19 & - & - & - & - & - & - \\
\hline $2125-2112$ & S16 & December 2000 & 22 & 20 & - & - & - & - & - & - \\
\hline $2126-2100$ & S17 & July 1997 & 7.3 & 0.85 & - & - & - & - & - & - \\
\hline $2126-2100$ & S17 & September 1998 & 15.0 & 14.0 & - & - & - & - & - & - \\
\hline $2126-2100$ & S17 & November 1999 & 17 & 12 & - & - & - & - & - & - \\
\hline $2126-2100$ & S17 & November 1999 & 16 & & - & - & - & - & - & - \\
\hline $2126-2100$ & S17 & December 2000 & 18 & 15 & - & - & - & - & - & - \\
\hline $2126-2136$ & S18 & July 1997 & 15 & 3.9 & - & - & - & - & - & - \\
\hline $2126-2136$ & S18 & September 1998 & 22.0 & 29.0 & - & - & - & - & - & - \\
\hline $2126-2136$ & S18 & November 1999 & 20 & 24 & - & - & - & - & - & - \\
\hline $2126-2136$ & $\mathrm{~S} 18$ & December 2000 & 19 & 26 & - & - & - & - & - & - \\
\hline $2129-2203$ & S19 & September 1998 & 99.0 & 95.0 & - & - & - & - & - & - \\
\hline $2129-2203$ & S19 & November 1999 & 31 & 100 & - & - & - & - & - & 2.9 \\
\hline $2129-2203$ & S19 & December 2000 & 54 & 63 & 26 & 5 & 2.3 & 2 & 1.9 & \\
\hline $2132-2144$ & $\mathrm{~S} 20$ & September 1998 & 13.0 & 21.0 & - & - & - & - & - & - \\
\hline $2132-2144$ & S20 & November 1999 & 11 & 21 & - & - & - & - & - & - \\
\hline $2132-2144$ & S20 & December 2000 & 12 & 11 & - & - & - & - & - & - \\
\hline $2123-2130$ & & July 1997 & 15 & 8.8 & - & - & - & - & - & - \\
\hline $2051-2158$ & & July 1997 & 9.3 & 8.4 & - & - & - & - & - & - \\
\hline $2113-2120$ & & July 1997 & 8.1 & 13 & - & - & - & - & - & - \\
\hline $2117-2107$ & & July 1997 & 12 & 14 & - & - & - & - & - & - \\
\hline $2121-2120$ & & July 1997 & 16 & 7.1 & - & - & - & - & - & - \\
\hline $2123-2411$ & & July 1997 & 50 & 13 & - & - & - & - & - & - \\
\hline $2124-2211$ & & July 1997 & 92 & 65 & - & - & - & - & - & - \\
\hline $2125-2113$ & & July 1997 & 7.8 & 4.1 & - & - & - & - & - & - \\
\hline $2128-2145$ & & July 1997 & 18 & 14 & - & - & - & - & - & - \\
\hline $2131-2159$ & & July 1997 & 4.5 & 2.5 & - & - & - & - & - & - \\
\hline J3-4097 & & July 1997 & 19 & 24 & - & - & - & - & - & - \\
\hline J3-8097 & & July 1997 & 18 & 8.8 & - & - & - & - & - & - \\
\hline J4-3097 & & July 1997 & 54 & 310 & - & - & - & - & - & - \\
\hline J4-6097 & & July 1997 & 67 & 54 & - & - & - & - & - & - \\
\hline
\end{tabular}


Table 25. Concentration of selenium species in bottom-sediment samples collected from experimental test plots and selected sites at Stewart Lake near Jensen, Utah, April 2000-August 2000

[Data from Bureau of Reclamation; All selenium values in micrograms per gram, dry weight; - , no data]

\begin{tabular}{|c|c|c|c|c|c|c|c|}
\hline $\begin{array}{c}\text { Site ID } \\
\text { number }\end{array}$ & $\begin{array}{l}\text { Permanent } \\
\text { site } \\
\text { ID }\end{array}$ & Date & $\begin{array}{c}\text { Depth } \\
\text { below } \\
\text { land } \\
\text { surface } \\
\text { (inches) }\end{array}$ & $\begin{array}{c}\text { Selenite, } \\
\text { soluble } \\
(\mathrm{Se}(\mathrm{IV}))\end{array}$ & $\begin{array}{c}\text { Selenate, } \\
\text { soluble } \\
(\mathrm{Se}(\mathrm{VI}))\end{array}$ & $\begin{array}{c}\text { Selenium, } \\
\text { soluble } \\
\text { organic } \\
\text { (Se) }\end{array}$ & $\begin{array}{c}\text { Selenite, } \\
\text { insoluble } \\
(\mathrm{Se}(\mathrm{IV}))\end{array}$ \\
\hline \multirow[t]{11}{*}{$2048-2149$} & S-1 Till plot & April 2000 & $0-6$ & 0.34 & 0.28 & 0.07 & 4.20 \\
\hline & S-1 Till plot & April 2000 & $4-14$ & .21 & .57 & .04 & 2.64 \\
\hline & S-1 Till plot & April 2000 & $18-24$ & .06 & .15 & .01 & .41 \\
\hline & S-1 Till plot & August 2000 & $0-6$ & .39 & .46 & .02 & 4.40 \\
\hline & S-1 Till plot & August 2000 & $4-14$ & .14 & .27 & .02 & 1.80 \\
\hline & S-1 Till plot & August 2000 & $18-24$ & .10 & .12 & .01 & .64 \\
\hline & S-1 Box Plot & August 2000 & $0-3$ & .31 & .38 & .03 & 5.24 \\
\hline & S-1 Box Plot & August 2000 & $3-6$ & .29 & .35 & .03 & 5.53 \\
\hline & S-1 Control & August 2000 & $0-6$ & .32 & .36 & .04 & 5.41 \\
\hline & S-1 Control & August 2000 & $6-14$ & .12 & .24 & .03 & 1.95 \\
\hline & S-1 Control & August 2000 & $18-24$ & .08 & .11 & .01 & .80 \\
\hline \multirow[t]{7}{*}{ 2057-2201 } & S-5 & April 2000 & $0-4$ & .18 & 2.07 & .06 & 2.29 \\
\hline & S-5 & April 2000 & $4-10$ & .06 & .61 & .05 & 1.10 \\
\hline & S-5 & April 2000 & $4-10$ & .06 & .62 & .06 & 1.54 \\
\hline & S-5 & April 2000 & 40 & .02 & .02 & .01 & .16 \\
\hline & S-5 & August 2000 & $0-4$ & .07 & .34 & .02 & 1.59 \\
\hline & S-5 & August 2000 & $4-10$ & .09 & .38 & .04 & 1.41 \\
\hline & S-5 & August 2000 & 40 & .03 & .03 & .00 & .13 \\
\hline \multirow[t]{11}{*}{ 2113-2119 } & S-10 Till plot & April 2000 & $0-2$ & .28 & .53 & .04 & 1.80 \\
\hline & S-10 Till plot & April 2000 & $4-8$ & .28 & .62 & .03 & 2.50 \\
\hline & S-10 Till plot & April 2000 & $8-12$ & .48 & .21 & .02 & 3.52 \\
\hline & S-10 Till plot & August 2000 & $0-2$ & .29 & .51 & .03 & 1.66 \\
\hline & S-10 Till plot & August 2000 & $4-8$ & .34 & .31 & .02 & 4.53 \\
\hline & S-10 Till plot & August 2000 & $8-12$ & .07 & .05 & .01 & .74 \\
\hline & S-10 Box plot & August 2000 & $0-3$ & .35 & .69 & .01 & 2.06 \\
\hline & S-10 Box plot & August 2000 & $3-6$ & .33 & .42 & .02 & 2.84 \\
\hline & S-10 Control & August 2000 & $0-3$ & .31 & .58 & .06 & 1.47 \\
\hline & S-10 Control & August 2000 & $12-15$ & .17 & .24 & .03 & 2.83 \\
\hline & S-10 Control & August 2000 & $18-21$ & .11 & .12 & .01 & 1.02 \\
\hline \multirow[t]{14}{*}{$2133-2144$} & S-15 Till plot & April 2000 & $0-4$ & 1.31 & 3.56 & .25 & 5.90 \\
\hline & S-15 Till plot & April 2000 & $0-4$ & .83 & 4.38 & .16 & 5.56 \\
\hline & S-15 Till plot & April 2000 & 4-12 & .53 & 1.53 & .07 & 4.44 \\
\hline & S-15 Till plot & April 2000 & $15-30$ & .22 & .14 & .04 & 1.73 \\
\hline & S-15 Till plot & April 2000 & $15-30$ & .59 & 1.25 & .08 & 4.16 \\
\hline & S-15 Till plot & August 2000 & $0-4$ & 1.04 & 1.46 & .15 & 5.50 \\
\hline & S-15 Till plot & August 2000 & $4-12$ & .48 & 1.21 & .06 & 4.39 \\
\hline & S-15 Till plot & August 2000 & $0-4$ & .89 & 1.68 & .17 & 5.79 \\
\hline & S-15 Till plot & August 2000 & $15-30$ & .43 & .23 & .04 & 3.45 \\
\hline & S-15 Box plot & August 2000 & $0-3$ & .91 & 1.57 & .1 & 6.20 \\
\hline & S-15 Box plot & August 2000 & $3-6$ & .82 & 1.45 & .10 & 6.28 \\
\hline & S-15 Control & August 2000 & $0-4$ & .64 & 1.36 & .08 & 5.05 \\
\hline & S-15 Control & August 2000 & 4-10 & .53 & .61 & .04 & 5.03 \\
\hline & S-15 Control & August 2000 & $15-30$ & .42 & .22 & .04 & 3.04 \\
\hline \multirow[t]{6}{*}{$2129-2203$} & S-19 & April 2000 & $0-4$ & 1.57 & 2.34 & .26 & 11.78 \\
\hline & S-19 & April 2000 & $6-18$ & .53 & .16 & .04 & 2.81 \\
\hline & S-19 & April 2000 & $18-30$ & .07 & .04 & .03 & .52 \\
\hline & S-19 & August 2000 & $0-4$ & 1.40 & 2.83 & .33 & 6.81 \\
\hline & S-19 & August 2000 & 6-18 & .67 & .5 & .06 & 4.84 \\
\hline & S-19 & August 2000 & $18-30$ & .41 & .09 & .03 & 2.31 \\
\hline
\end{tabular}


Table 25. Concentration of selenium species in bottom-sediment samples collected from experimental test plots and selected sites at Stewart Lake near Jensen, Utah, April 2000-August 2000-Continued

\begin{tabular}{|c|c|c|c|c|c|c|c|c|}
\hline $\begin{array}{l}\text { Permanent site } \\
\text { ID }\end{array}$ & $\begin{array}{c}\text { Selenite, } \\
\text { insoluble } \\
(\mathrm{Se}(\mathrm{VI}))\end{array}$ & $\begin{array}{c}\text { Selenium, } \\
\text { insoluble } \\
\text { organic } \\
\text { (Se) }\end{array}$ & $\begin{array}{l}\text { Selenide and } \\
\text { Elemental } \\
\text { Selenium } \\
(\mathrm{Se}(-\mathrm{II})+ \\
\mathrm{Se}(0))\end{array}$ & $\begin{array}{l}\text { Selenium, } \\
\text { related } \\
\text { organic } \\
\text { materials } \\
(\mathrm{Se})\end{array}$ & $\begin{array}{l}\text { Selenium, } \\
\text { residue } \\
\text { (Se) }\end{array}$ & $\begin{array}{l}\text { Selenium, } \\
\text { total } \\
\text { soluble } \\
\text { (Se) }\end{array}$ & $\begin{array}{c}\text { Selenium, } \\
\text { total } \\
\text { insoluble } \\
\text { (Se) }\end{array}$ & $\begin{array}{c}\text { Selenium, } \\
\text { total } \\
\text { (Se) }\end{array}$ \\
\hline S-1 Till plot & 0.18 & 1.98 & 4.01 & & 0.37 & 0.69 & 10.36 & 11.42 \\
\hline S-1 Till plot & .00 & .71 & 3.11 & & .02 & .82 & 6.46 & 7.30 \\
\hline S-1 Till plot & .03 & .10 & .49 & & 0 & .22 & 1.02 & 1.24 \\
\hline S-1 Till plot & .50 & .59 & 5.16 & 5.80 & .47 & .87 & 11.56 & 12.62 \\
\hline S-1 Till plot & .06 & .22 & 2.37 & 2.60 & .01 & .42 & 8.76 & 5.10 \\
\hline S-1 Till plot & .03 & .07 & 1.15 & 1.23 & .08 & .23 & 7.55 & 2.27 \\
\hline S-1 Box Plot & .44 & .72 & 4.54 & 5.29 & .24 & .71 & 10.94 & 12.63 \\
\hline S-1 Box Plot & .36 & .81 & 4.51 & 5.36 & .05 & .67 & 10.91 & 12.77 \\
\hline S-1 Control & .37 & 1.58 & 4.50 & 6.12 & .22 & .72 & 10.90 & 14.42 \\
\hline S-1 Control & - & .15 & 2.61 & 2.79 & .15 & .39 & 9.01 & 5.42 \\
\hline S-1 Control & .18 & .12 & 1.16 & 1.30 & .01 & .21 & 7.56 & 2.61 \\
\hline S-5 & .00 & .77 & 3.17 & & .01 & 2.31 & 6.23 & 8.55 \\
\hline S-5 & .07 & .18 & 2.49 & & .11 & .71 & 3.85 & 4.67 \\
\hline S-5 & .14 & .30 & 3.01 & & .30 & .74 & 4.98 & 6.02 \\
\hline S-5 & .02 & .00 & .10 & & .05 & .05 & .28 & .38 \\
\hline S-5 & .09 & .25 & 2.83 & 3.10 & .18 & .43 & 9.22 & 5.63 \\
\hline S-5 & - & .46 & 3.05 & 3.55 & .70 & .52 & 9.45 & 6.6 \\
\hline S-5 & .00 & .01 & .13 & .15 & .03 & .07 & 6.53 & .39 \\
\hline S-10 Till plot & .26 & .04 & 2.87 & & .01 & .86 & 4.96 & 5.83 \\
\hline S-10 Till plot & .14 & .17 & 3.83 & & .02 & .93 & 6.64 & 7.59 \\
\hline S-10 Till plot & .00 & .44 & 9.92 & & .92 & .71 & 13.88 & 15.51 \\
\hline S-10 Till plot & .07 & .03 & 2.69 & 2.75 & .38 & .83 & 9.08 & 5.71 \\
\hline S-10 Till plot & .13 & .29 & 12.05 & 12.36 & .57 & .67 & 18.44 & 18.55 \\
\hline S-10 Till plot & .06 & .04 & .81 & .87 & .34 & .14 & 7.20 & 2.19 \\
\hline S-10 Box plot & .22 & .05 & 3.64 & 3.70 & .34 & 1.05 & 10.03 & 7.42 \\
\hline S-10 Box plot & .44 & .08 & 6.02 & 6.12 & .19 & .76 & 12.41 & 10.43 \\
\hline S-10 Control & - & .05 & 2.86 & 2.97 & .27 & .94 & 9.26 & 5.70 \\
\hline S-10 Control & .00 & .25 & 5.73 & 6.00 & .39 & .44 & 12.12 & 9.90 \\
\hline S-10 Control & .18 & 0 & 1.36 & 1.37 & .03 & .23 & 7.75 & 2.83 \\
\hline S-15 Till plot & .57 & 1.36 & 9.32 & & 1.37 & 5.14 & 17.15 & 23.67 \\
\hline S-15 Till plot & .05 & 1.41 & 11.08 & & 2.22 & 5.36 & 18.10 & 25.68 \\
\hline S-15 Till plot & .00 & .69 & 8.42 & & 1.63 & 2.13 & 13.55 & 17.31 \\
\hline S-15 Till plot & .20 & .19 & 1.38 & & .25 & .40 & 3.49 & 4.15 \\
\hline S-15 Till plot & .18 & .50 & 8.94 & & 1.03 & 1.91 & 13.78 & 16.72 \\
\hline S-15 Till plot & .39 & .52 & 11.17 & 11.84 & .59 & 2.65 & 17.56 & 21.49 \\
\hline S-15 Till plot & .16 & .64 & 9.94 & 10.64 & .28 & 1.76 & 16.33 & 17.86 \\
\hline S-15 Till plot & .31 & .31 & 11.02 & 11.51 & .01 & 2.74 & 17.42 & 20.66 \\
\hline S-15 Till plot & .28 & .32 & 3.28 & 3.64 & .03 & .70 & 9.68 & 8.42 \\
\hline S-15 Box plot & .39 & .31 & 11.33 & 11.74 & .99 & 2.58 & 17.72 & 22.22 \\
\hline S-15 Box plot & .39 & .64 & 11.36 & 12.09 & 1.23 & 2.37 & 17.75 & 22.99 \\
\hline S-15 Control & - & .48 & 11.44 & 12.01 & .69 & 2.07 & 17.83 & 20.28 \\
\hline S-15 Control & .12 & .62 & 11.58 & 12.25 & .77 & 1.18 & 17.98 & 19.96 \\
\hline S-15 Control & .45 & .48 & 2.60 & 3.12 & .45 & .67 & 8.99 & 8.21 \\
\hline S-19 & .00 & 2.80 & 17.75 & & 4.00 & 4.17 & 32.32 & 40.49 \\
\hline S-19 & .01 & .13 & 4.33 & & .22 & .73 & 7.28 & 8.23 \\
\hline S-19 & .07 & .01 & .42 & & .09 & .13 & 1.02 & 1.24 \\
\hline S-19 & .08 & .98 & 17.22 & 18.53 & .14 & 4.56 & 23.61 & 31.09 \\
\hline S-19 & .12 & .10 & 9.09 & 9.25 & .57 & 1.23 & 15.48 & 16.12 \\
\hline S-19 & .02 & .05 & 2.81 & 2.89 & .44 & .53 & 9.2 & \\
\hline
\end{tabular}


Table 26. Concentration of total selenium in bottom-sediment samples collected from experimental test plots and selected sites at Stewart Lake near Jensen, Utah, April 2000-December 2000

[Data from Bureau of Reclamation; - , no data]

\begin{tabular}{|c|c|c|c|c|c|c|c|c|}
\hline \multirow{3}{*}{ Permanent site ID } & \multirow{3}{*}{ Date } & \multicolumn{7}{|c|}{ Depth below land surface (inches) } \\
\hline & & $0-3$ & $3-6$ & $6-12$ & $12-18$ & $18-24$ & $24-30$ & $30-36$ \\
\hline & & \multicolumn{7}{|c|}{ Selenium, total (micrograms per gram, dry weight) } \\
\hline \multirow[t]{3}{*}{ S-1 Till plot } & April 2000 & 11 & 7.6 & 5.9 & 6.1 & 2.7 & 1.8 & 2.9 \\
\hline & August 2000 & 14 & 12 & 7.6 & 6.6 & 2.5 & 1.9 & 1.7 \\
\hline & December 2000 & 12 & 14 & 9.6 & 5.1 & 1.9 & 2 & 2.2 \\
\hline \multirow[t]{2}{*}{ S-1 Box plot } & August 2000 & 15 & 15 & - & - & - & - & - \\
\hline & December 2000 & 16 & 16 & - & - & - & - & - \\
\hline \multirow[t]{3}{*}{ S-1 Control } & April 2000 & 9.9 & 10 & 4.6 & 4.6 & 2.7 & 2.5 & 1.7 \\
\hline & August 2000 & 14 & 18 & 12 & 6.2 & 2.6 & 2.2 & 1.7 \\
\hline & December 2000 & 12 & 21 & 16 & 5.2 & 2.6 & 2.6 & 2.1 \\
\hline \multirow[t]{3}{*}{ S-5 } & April 2000 & 7.7 & 6.6 & 5.2 & 3.2 & 2.2 & 1.1 & .68 \\
\hline & August 2000 & 7.8 & 8.1 & 5.5 & 2.6 & 1.8 & 1.1 & .61 \\
\hline & December 2000 & 7.2 & 8 & 6.7 & 2.8 & 1.7 & .8 & .6 \\
\hline \multirow[t]{3}{*}{ S-10 Till plot } & April 2000 & 8.9 & 13 & 22 & 15 & 3.3 & 1.1 & .68 \\
\hline & August 2000 & 8.3 & 15 & 24 & 19 & 4.2 & 1 & .68 \\
\hline & December 2000 & 9.2 & 10 & 20 & 13 & 2.8 & 1.2 & .58 \\
\hline \multirow[t]{2}{*}{ S-10 Box plot } & August 2000 & 9.7 & 10 & - & - & - & - & - \\
\hline & December 2000 & 8.7 & 11 & - & - & - & - & - \\
\hline \multirow[t]{2}{*}{ S-10 Control } & August 2000 & 8.9 & 16 & 18 & 9.8 & 3 & 1.4 & .94 \\
\hline & December 2000 & 6.2 & 14 & 13 & 5.1 & 1.4 & .64 & .56 \\
\hline \multirow[t]{3}{*}{ S-15 Till plot } & April 2000 & 16 & 10 & 8.2 & 2.9 & 2.9 & 9.4 & 1.8 \\
\hline & August 2000 & 28 & 18 & 13 & 3.2 & 3.1 & 8.2 & 4.2 \\
\hline & December 2000 & 32 & 25 & 8.6 & 4.5 & 5.6 & 8.2 & 3.1 \\
\hline \multirow[t]{2}{*}{ S-15 Box plot } & August 2000 & 27 & 28 & - & - & - & - & - \\
\hline & December 2000 & 27 & 28 & - & - & - & - & - \\
\hline \multirow[t]{2}{*}{ S-15 Control } & August 2000 & 32 & 28 & 20 & 7.9 & 6.6 & 11 & 4.5 \\
\hline & December 2000 & 31 & 26 & 7.4 & 5.9 & 5.6 & 9 & 3.9 \\
\hline \multirow[t]{3}{*}{ S-19 } & April 2000 & 19 & 59 & 11 & 1.7 & 1.7 & 1.5 & 1.9 \\
\hline & August 2000 & 32 & 53 & 53 & 13 & 3.3 & 2.3 & 2.1 \\
\hline & December 2000 & 54 & 63 & 26 & 5 & 2.3 & 2 & 1.9 \\
\hline
\end{tabular}


Table 27. Trace-element concentration in whole body tissue composite samples of common carp collected from the Green River near Jensen, Utah, 1995 [Data from U.S. Fish and Wildlife Service; g, grams; $\mu \mathrm{g} / \mathrm{g}$, micrograms per gram, dry weight; <, less than; -, not reported]

\begin{tabular}{|c|c|c|c|c|c|c|c|c|c|c|c|c|}
\hline $\begin{array}{l}\text { Abbreviated } \\
\text { site name }\end{array}$ & Date & $\begin{array}{l}\text { Number of } \\
\text { fish }\end{array}$ & $\begin{array}{l}\text { Total weight } \\
\text { (g) }\end{array}$ & $\begin{array}{l}\text { Percent } \\
\text { moisture }\end{array}$ & $\begin{array}{l}\text { Aluminum } \\
(\mu \mathrm{g} / \mathrm{g})\end{array}$ & $\begin{array}{l}\text { Arsenic } \\
(\mu \mathrm{g} / \mathrm{g})\end{array}$ & $\begin{array}{l}\text { Boron } \\
(\mu \mathrm{g} / \mathrm{g})\end{array}$ & $\begin{array}{c}\text { Barium } \\
(\mu \mathrm{g} / \mathrm{g})\end{array}$ & $\begin{array}{l}\text { Beryllium } \\
\quad(\mu \mathrm{g} / \mathrm{g})\end{array}$ & $\begin{array}{c}\text { Cadmium } \\
(\mu \mathrm{g} / \mathrm{g})\end{array}$ & $\begin{array}{l}\text { Chromium } \\
(\mu \mathrm{g} / \mathrm{g})\end{array}$ & $\begin{array}{l}\text { Copper } \\
(\mu \mathrm{g} / \mathrm{g})\end{array}$ \\
\hline SA & $7-18-1995$ & 3 & 3,944 & 74.8 & 67.3 & $<1.00$ & $<4.02$ & 7.06 & $<0.201$ & 0.232 & 18.6 & 1.43 \\
\hline SA & 7-18-1995 & 4 & 3,807 & 74.4 & & & & & & & & \\
\hline SA & 8-28-1995 & 3 & 5,401 & 74.4 & 81.6 & $<.988$ & $<3.95$ & 6.52 & $<.198$ & .363 & 3.58 & 3.49 \\
\hline SA & 8-28-1995 & 3 & 3,811 & 72.8 & & & & & & & & \\
\hline $\mathrm{BB}$ & 8-29-1995 & 3 & 3,607 & 74.3 & 146 & $<.965$ & $<3.86$ & 1.36 & $<.193$ & .464 & 8.32 & 4.42 \\
\hline $\mathrm{BB}$ & $8-29-1995$ & 3 & 4,934 & 72.9 & - & - & - & - & - & - & - & - \\
\hline $\mathrm{BB}$ & 8-29-1995 & 3 & 4,847 & 74.7 & - & - & - & - & - & - & - & - \\
\hline $\mathrm{BC}$ & 7-18-1995 & 5 & 4,935 & 74.5 & 46.8 & $<.965$ & $<3.86$ & 1.24 & $<.193$ & .604 & 5.29 & 3.67 \\
\hline $\mathrm{BC}$ & 7-18-1995 & 5 & 4,417 & 77.2 & & & & & & & & \\
\hline $\mathrm{BC}$ & 8-28-1995 & 3 & 2,670 & 75.1 & 127.8 & $<1.01$ & $<4.05$ & 9.64 & $<.202$ & .356 & 4.45 & 3.40 \\
\hline $\mathrm{BC}$ & 8-28-1995 & 3 & 5,246 & 73.4 & & & & & & & & \\
\hline $\mathrm{BO}$ & $7-19-1995$ & 3 & 2,428 & 74.2 & - & - & - & - & - & - & - & - \\
\hline $\mathrm{BO}$ & 7-19-1995 & 3 & 2,077 & 74.8 & 8.1 & $<.962$ & $<3.85$ & 9.68 & $<.192$ & $<.192$ & 5.54 & 2.74 \\
\hline $\mathrm{BO}$ & 8-28-1995 & 3 & 3,455 & 74.9 & - & - & - & - & - & - & - & - \\
\hline $\mathrm{BO}$ & 8-28-1995 & 3 & 1,506 & 75.2 & 74.8 & $<.977$ & $<3.91$ & 2.34 & $<.195$ & $<.195$ & 9.19 & 1.24 \\
\hline $\mathrm{BO}$ & 8-28-1995 & 4 & 4,094 & 73.2 & 93.7 & $<.992$ & $<3.97$ & 8.44 & $<.198$ & .327 & 4.54 & 3.31 \\
\hline $\mathrm{CD}$ & 8-29-1995 & 3 & 4,457 & 72.2 & 89.1 & $<.965$ & $<3.86$ & 7.35 & $<.193$ & .449 & 19.2 & 3.43 \\
\hline $\mathrm{CD}$ & 8-29-1995 & 3 & 5,401 & 74.4 & - & - & - & - & - & - & - & - \\
\hline $\mathrm{CD}$ & $8-29-1995$ & 3 & 6,230 & 72.0 & - & - & - & - & - & - & - & - \\
\hline EB & $7-18-1995$ & 5 & 4,702 & 76.4 & 58.0 & $<.988$ & $<3.95$ & 6.43 & $<.198$ & .566 & 67.8 & 3.66 \\
\hline EB & 7-18-1995 & 5 & 5,834 & 74.7 & - & - & - & - & - & - & - & - \\
\hline $\mathrm{EB}$ & 8-28-1995 & 3 & 5,247 & 72.8 & 72.2 & $<.969$ & $<3.88$ & 8.36 & $<.194$ & .362 & 4.13 & 2.56 \\
\hline $\mathrm{EB}$ & 8-28-1995 & 3 & 4,637 & 73.0 & & & & & & & & - \\
\hline HA & 8-31-1995 & 3 & 3,713 & 72.9 & 120 & $<.984$ & $<3.94$ & 10.0 & $<.197$ & .695 & 9.54 & 4.74 \\
\hline HA & 8-31-1995 & 3 & 5,047 & 70.8 & - & - & - & - & - & - & - & - \\
\hline HA & 8-31-1995 & 3 & 5,458 & 71.4 & - & - & - & - & - & - & - & - \\
\hline $\mathrm{HB}$ & 8-29-1995 & 3 & 4,865 & 73.1 & 123 & $<.977$ & $<3.91$ & 9.46 & $<.195$ & .432 & 18.1 & 4.09 \\
\hline $\mathrm{HB}$ & $8-29-1995$ & 3 & 6,326 & 72.1 & - & - & - & - & - & - & - & - \\
\hline LB & 8-30-1995 & 3 & 1,513 & 77.6 & 386 & $<.973$ & $<3.89$ & 11.2 & $<.195$ & $<.195$ & 12.3 & 5.64 \\
\hline LB & 8-30-1995 & 3 & 3,916 & 72.3 & - & - & - & - & - & - & - & - \\
\hline LB & 8-30-1995 & 3 & 3,772 & 73.8 & - & - & - & - & - & - & - & - \\
\hline SB & $7-18-1995$ & 3 & 4,282 & 74.8 & 170 & $<.969$ & $<3.88$ & 11.3 & $<.194$ & .465 & 9.83 & 4.39 \\
\hline SB & 7-18-1995 & 3 & 5,690 & 72.9 & - & - & - & - & - & - & - & - \\
\hline SB & 7-18-1995 & 3 & 6,268 & 71.7 & - & - & - & - & - & - & - & - \\
\hline
\end{tabular}


Table 27. Trace-element concentration in whole body tissue composite samples of common carp collected from the Green River near Jensen, Utah, 1995 -Continued

\begin{tabular}{|c|c|c|c|c|c|c|c|c|c|c|c|}
\hline $\begin{array}{l}\text { Abbreviated } \\
\text { site name }\end{array}$ & $\begin{array}{l}\text { Iron } \\
(\mu \mathrm{g} / \mathrm{g})\end{array}$ & $\begin{array}{c}\text { Mercury } \\
(\mu \mathrm{g} / \mathrm{g})\end{array}$ & $\underset{(\mu \mathrm{g} / \mathrm{g})}{\text { Magnesium }}$ & $\begin{array}{c}\text { Manganese } \\
(\mu \mathrm{g} / \mathrm{g})\end{array}$ & $\begin{array}{l}\text { Molybdenum } \\
(\mu \mathrm{g} / \mathrm{g})\end{array}$ & $\begin{array}{l}\text { Nickel } \\
(\mu \mathrm{g} / \mathrm{g})\end{array}$ & $\begin{array}{l}\text { Lead } \\
(\mu \mathrm{g} / \mathrm{g})\end{array}$ & $\begin{array}{c}\text { Selenium } \\
(\mu \mathrm{g} / \mathrm{g})\end{array}$ & $\begin{array}{c}\text { Strontium } \\
(\mu \mathrm{g} / \mathrm{g})\end{array}$ & $\begin{array}{c}\text { Vanadium } \\
(\mu \mathrm{g} / \mathrm{g})\end{array}$ & $\begin{array}{l}\text { Zinc } \\
(\mu \mathrm{g} / \mathrm{g})\end{array}$ \\
\hline SA & 298 & $<0.201$ & 1,150 & 51.7 & $<1.00$ & 3.00 & $<2.01$ & 36.1 & 160 & $<1.00$ & 284 \\
\hline SA & & & & & & & & 11.4 & & & \\
\hline SA & 268 & $<.198$ & 1,220 & 12.2 & $<.988$ & $<.988$ & $<1.98$ & 18.0 & 128 & $<.988$ & 215 \\
\hline SA & & & & & & & & 19.9 & & & \\
\hline BB & 397 & .384 & 1,380 & 15.4 & $<.965$ & 1.44 & $<1.93$ & 13.2 & 127 & $<.965$ & 258 \\
\hline BB & - & - & - & - & - & - & - & 9.45 & - & - & - \\
\hline BB & - & - & - & - & - & - & - & 16.2 & - & - & - \\
\hline $\mathrm{BC}$ & 213 & .343 & 1,300 & 9.40 & $<.965$ & 1.01 & $<1.93$ & 6.24 & 136 & $<.965$ & 231 \\
\hline $\mathrm{BC}$ & & & & & & & & 8.03 & & & \\
\hline $\mathrm{BC}$ & 305 & .252 & 1,490 & 15.7 & $<1.01$ & $<1.01$ & $<2.02$ & 13.7 & 174 & $<1.01$ & 236 \\
\hline $\mathrm{BC}$ & & & & & & & & 6.80 & & & \\
\hline BO & - & - & - & - & - & - & - & 13.9 & - & - & - \\
\hline $\mathrm{BO}$ & 168 & $<.192$ & 1,730 & 11.9 & $<.962$ & $<.962$ & $<1.92$ & 27.2 & 256 & $<.962$ & 281 \\
\hline $\mathrm{BO}$ & - & - & - & - & - & - & - & 14.0 & - & - & - \\
\hline BO & 186 & .484 & 1,070 & 8.71 & $<.977$ & $<.977$ & $<1.95$ & 2.93 & 66.3 & $<.977$ & 90.0 \\
\hline BO & 245 & .271 & 1,300 & 12.3 & $<.992$ & $<.992$ & $<1.98$ & 13.8 & 132 & $<.992$ & 242 \\
\hline $\mathrm{CD}$ & 348 & .334 & 1,080 & 10.9 & $<.965$ & 2.06 & $<1.93$ & 7.34 & 103 & $<.965$ & 196 \\
\hline $\mathrm{CD}$ & - & - & - & - & - & - & - & 12.5 & - & - & - \\
\hline $\mathrm{CD}$ & - & - & - & - & - & - & - & 9.22 & - & - & - \\
\hline EB & 800 & .232 & 1,120 & 14.6 & 1.40 & 11.8 & $<1.98$ & 27.7 & 116 & $<.988$ & 380 \\
\hline EB & - & - & - & - & - & - & - & 6.04 & - & - & - \\
\hline EB & 196 & .422 & 1,250 & 8.58 & $<.969$ & $<.969$ & $<1.94$ & 7.93 & 126 & $<.969$ & 264 \\
\hline EB & - & - & - & - & - & - & - & 15.7 & - & - & - \\
\hline HA & 302 & .425 & 1,680 & 11.9 & $<.984$ & 1.16 & $<1.97$ & 5.47 & 144 & $<.984$ & 265 \\
\hline HA & - & - & - & - & - & - & - & 3.33 & - & - & - \\
\hline HA & - & - & - & - & - & - & - & 5.26 & - & - & - \\
\hline HB & 331 & .358 & 1,260 & 10.9 & $<.977$ & 2.02 & $<1.96$ & 5.43 & 104 & $<.977$ & 292 \\
\hline HB & - & - & - & - & - & - & - & 3.82 & - & - & - \\
\hline LB & 621 & $<.195$ & 1,970 & 20.7 & $<.973$ & 2.90 & $<1.96$ & 20.9 & 190 & 1.18 & 334 \\
\hline LB & - & - & - & - & - & - & - & 4.10 & - & - & - \\
\hline LB & - & - & - & - & - & - & - & 3.95 & - & - & - \\
\hline SB & 428 & .349 & 1,530 & 15.4 & $<.969$ & 1.83 & $<1.94$ & 8.39 & 140 & $<.969$ & 427 \\
\hline SB & - & - & - & - & - & - & - & 8.68 & - & - & - \\
\hline SB & - & - & - & - & - & - & - & 4.90 & - & - & - \\
\hline
\end{tabular}


Table 28. Concentration of selenium in whole body tissue composite samples of common carp collected from the Green River near Jensen, Utah, 1996-2000

[Data from U.S. Fish and Wildlife Service; $\mu \mathrm{g} / \mathrm{g}$, micrograms per gram, dry weight]

\begin{tabular}{|c|c|c|c|c|c|}
\hline $\begin{array}{l}\text { Abbreivated } \\
\text { site name }\end{array}$ & Date & $\begin{array}{l}\text { Number of } \\
\text { fish }\end{array}$ & $\begin{array}{l}\text { Total weight } \\
\text { (grams) }\end{array}$ & $\begin{array}{l}\text { Percent } \\
\text { moisture }\end{array}$ & $\begin{array}{l}\text { Selenium } \\
(\mu \mathrm{g} / \mathrm{g})\end{array}$ \\
\hline $\mathrm{BB}$ & 08-06-1997 & 3 & 4,857 & 71.6 & 4.12 \\
\hline BB & 08-06-1997 & 3 & 3,017 & 73.7 & 11.5 \\
\hline $\mathrm{BB}$ & 08-06-1997 & 3 & 1,983 & 76.5 & 19.8 \\
\hline $\mathrm{BB}$ & 08-12-1998 & 4 & 4,916 & 73.7 & 7.6 \\
\hline $\mathrm{BB}$ & 08-12-1998 & 3 & 2,771 & 75.7 & 11.4 \\
\hline $\mathrm{BB}$ & 08-12-1998 & 4 & 1,529 & 76.9 & 12.3 \\
\hline $\mathrm{BB}$ & 03-17-1999 & 5 & 2,109 & 79.4 & 13.4 \\
\hline BB & 03-17-1999 & 5 & 6,732 & 74.7 & 7.18 \\
\hline BB & 03-17-1999 & 4 & 3,299 & 77.4 & 13.1 \\
\hline BB & 08-04-1999 & 5 & 6,203 & 71.5 & 6.06 \\
\hline BB & 08-04-1999 & 5 & 2,259 & 75.6 & 9.73 \\
\hline $\mathrm{BB}$ & 08-04-1999 & 5 & 3,958 & 74.6 & 4.04 \\
\hline BB & 03-08-2000 & 5 & 2,329 & 74.7 & 7.68 \\
\hline BB & 03-08-2000 & 4 & 1,828 & 76.4 & 5.29 \\
\hline BB & 03-08-2000 & 5 & 1,949 & 78.4 & 2.36 \\
\hline $\mathrm{BB}$ & 08-08-2000 & 5 & 3,257 & 74.9 & 6.38 \\
\hline $\mathrm{BB}$ & 08-08-2000 & 5 & 5,284 & 71.8 & 4.01 \\
\hline BB & 08-08-2000 & 5 & 5,939 & 72.2 & 3.98 \\
\hline $\mathrm{BC}$ & 07-30-1996 & 5 & 4,922 & 71.0 & 5.0 \\
\hline $\mathrm{BC}$ & 07-30-1996 & 5 & 8,727 & 69.3 & 4.2 \\
\hline $\mathrm{BC}$ & 07-30-1996 & 5 & 2,812 & 74.8 & 24.1 \\
\hline $\mathrm{BC}$ & 08-05-1997 & 4 & 4,654 & 74.6 & 8.65 \\
\hline $\mathrm{BC}$ & 08-05-1997 & 4 & 3,152 & 75.2 & 14.2 \\
\hline $\mathrm{BC}$ & 08-05-1997 & 4 & 2,151 & 76.1 & 16.0 \\
\hline $\mathrm{BC}$ & 08-13-1998 & 3 & 3,107 & 78.9 & 8.43 \\
\hline $\mathrm{BC}$ & 08-13-1998 & 3 & 1,775 & 76.7 & 15.3 \\
\hline $\mathrm{BC}$ & 08-13-1998 & 5 & 3,483 & 79.7 & 18.3 \\
\hline $\mathrm{BC}$ & 03-17-1999 & 4 & 3,537 & 78.1 & 16.7 \\
\hline $\mathrm{BC}$ & 03-17-1999 & 4 & 4,571 & 77.6 & 5.80 \\
\hline $\mathrm{BC}$ & 03-17-1999 & 4 & 5,262 & 75.7 & 4.37 \\
\hline $\mathrm{BC}$ & 08-03-1999 & 5 & 6,100 & 72.1 & 7.79 \\
\hline $\mathrm{BC}$ & 08-03-1999 & 5 & 2,494 & 76.6 & 5.86 \\
\hline $\mathrm{BC}$ & 08-03-1999 & 5 & 4,274 & 76.4 & 3.77 \\
\hline $\mathrm{BC}$ & 03-07-2000 & 5 & 1,755 & 80.0 & 11.5 \\
\hline $\mathrm{BC}$ & 03-07-2000 & 5 & 7,191 & 78.1 & 5.18 \\
\hline $\mathrm{BC}$ & 03-07-2000 & 5 & 4,475 & 79.0 & 3.46 \\
\hline $\mathrm{BC}$ & 08-08-2000 & 5 & 2,778 & 76.7 & 11.2 \\
\hline $\mathrm{BC}$ & 08-08-2000 & 5 & 5,932 & 74.7 & 5.48 \\
\hline $\mathrm{BC}$ & 08-08-2000 & 5 & 4,712 & 75.1 & 13.4 \\
\hline BO & 08-05-1997 & 3 & 4,519 & 73.0 & 13.3 \\
\hline BO & 08-05-1997 & 3 & 3,229 & 71.3 & 9.08 \\
\hline BO & 08-05-1997 & 3 & 2,066 & 75.6 & 10.2 \\
\hline $\mathrm{BO}$ & 08-11-1998 & 4 & 4,771 & 68.5 & 2.83 \\
\hline $\mathrm{BO}$ & 08-11-1998 & 4 & 3,208 & 75.9 & 12.5 \\
\hline $\mathrm{BO}$ & 08-11-1998 & 4 & 2,765 & 75.8 & 15.0 \\
\hline $\mathrm{BO}$ & 03-17-1999 & 5 & 3,299 & 78.3 & 14.6 \\
\hline
\end{tabular}


Table 28. Concentration of selenium in whole body tissue composite samples of common carp collected from the Green River near Jensen, Utah, 1996-2000-Continued

\begin{tabular}{|c|c|c|c|c|c|}
\hline $\begin{array}{l}\text { Abbreivated } \\
\text { site name }\end{array}$ & Date & $\begin{array}{l}\text { Number of } \\
\text { fish }\end{array}$ & $\begin{array}{l}\text { Total weight } \\
\text { (grams) }\end{array}$ & $\begin{array}{l}\text { Percent } \\
\text { moisture }\end{array}$ & $\begin{array}{l}\text { Selenium } \\
(\mu \mathrm{g} / \mathrm{g})\end{array}$ \\
\hline $\mathrm{BO}$ & 03-17-1999 & 5 & 3,859 & 81.8 & 14.0 \\
\hline $\mathrm{BO}$ & 03-17-1999 & 5 & 5,896 & 77.0 & 9.74 \\
\hline $\mathrm{BO}$ & 08-05-1999 & 4 & 3,323 & 75.3 & 8.87 \\
\hline $\mathrm{BO}$ & 08-05-1999 & 4 & 4,566 & 75.3 & 5.47 \\
\hline $\mathrm{BO}$ & 03-08-2000 & 5 & 2,527 & 78.1 & 10.3 \\
\hline $\mathrm{BO}$ & $03-08-2000$ & 5 & 2,149 & 75.8 & 12.6 \\
\hline $\mathrm{BO}$ & 03-08-2000 & 5 & 2,075 & 76.9 & 13.6 \\
\hline $\mathrm{BO}$ & 08-08-2000 & 5 & 3,042 & 75.9 & 13.1 \\
\hline $\mathrm{BO}$ & 08-08-2000 & 5 & 4,317 & 76.4 & 20.9 \\
\hline $\mathrm{BO}$ & 08-08-2000 & 5 & 5,654 & 74.1 & 21.1 \\
\hline BY & $03-15-2000$ & 4 & 3,956 & 76.7 & 8.52 \\
\hline BY & $03-15-2000$ & 4 & 5,603 & 76.4 & 7.65 \\
\hline BY & $03-15-2000$ & 2 & 1,324 & 79.1 & 1.39 \\
\hline BY & $08-10-2000$ & 5 & 3,144 & 76.0 & 6.22 \\
\hline BY & $08-10-2000$ & 5 & 4,653 & 72.5 & 8.86 \\
\hline BY & $08-10-2000$ & 5 & 5,889 & 71.4 & 6.05 \\
\hline $\mathrm{CD}$ & 08-07-1997 & 5 & 5,242 & 72.8 & 5.51 \\
\hline $\mathrm{CD}$ & 08-07-1997 & 5 & 6,429 & 73.6 & 8.06 \\
\hline $\mathrm{CD}$ & 08-07-1997 & 5 & 3,755 & 75.7 & 12.1 \\
\hline $\mathrm{CD}$ & 08-07-1997 & 4 & 1,193 & 77.7 & 6.46 \\
\hline $\mathrm{CD}$ & 08-12-1998 & 3 & 2,908 & 74.0 & 2.75 \\
\hline $\mathrm{CD}$ & 08-12-1998 & 3 & 2,039 & 76.5 & 6.87 \\
\hline $\mathrm{CD}$ & 08-12-1998 & 3 & 882 & 77.7 & 2.28 \\
\hline $\mathrm{CD}$ & 03-16-1999 & 5 & 5,947 & 75.5 & 6.89 \\
\hline $\mathrm{CD}$ & 03-16-1999 & 5 & 3,059 & 78.1 & 9.52 \\
\hline $\mathrm{CD}$ & 03-16-1999 & 5 & 1,713 & 78.8 & 11.6 \\
\hline $\mathrm{CD}$ & 08-04-1999 & 3 & 3,522 & 73.8 & 2.81 \\
\hline $\mathrm{CD}$ & 08-04-1999 & 5 & 4,252 & 75.4 & 2.25 \\
\hline $\mathrm{CD}$ & 08-04-1999 & 4 & 1,999 & 76.5 & 5.34 \\
\hline $\mathrm{CD}$ & $03-08-2000$ & 5 & 1,405 & 80.2 & 5.38 \\
\hline $\mathrm{CD}$ & $03-08-2000$ & 4 & 3,008 & 77.9 & 8.85 \\
\hline $\mathrm{CD}$ & $03-08-2000$ & 5 & 6,358 & 73.0 & 2.37 \\
\hline $\mathrm{CD}$ & 08-09-2000 & 5 & 2,728 & 74.4 & 9.05 \\
\hline $\mathrm{CD}$ & 08-09-2000 & 5 & 6,673 & 71.8 & 3.76 \\
\hline $\mathrm{CD}$ & 08-09-2000 & 5 & 4,732 & 69.8 & 6.63 \\
\hline EB & 08-05-1997 & 4 & 4,073 & 73.0 & 6.59 \\
\hline EB & 08-05-1997 & 3 & 4,053 & 69.1 & 2.71 \\
\hline EB & 08-05-1997 & 4 & 2,141 & 76.4 & 15.4 \\
\hline EB & 08-11-1998 & 3 & 2,813 & 75.5 & 8.30 \\
\hline EB & 08-11-1998 & 3 & 2,462 & 74.7 & 7.27 \\
\hline EB & 08-11-1998 & 3 & 1,788 & 76.1 & 10.2 \\
\hline EB & 03-17-1999 & 5 & 7,541 & 74.5 & 6.20 \\
\hline EB & 03-17-1999 & 5 & 3,933 & 78.0 & 18.8 \\
\hline EB & 03-17-1999 & 5 & 5,790 & 76.5 & 9.24 \\
\hline EB & 08-03-1999 & 5 & 6,127 & 73.2 & 9.73 \\
\hline EB & 08-03-1999 & 3 & 1,040 & 78.2 & 10.5 \\
\hline EB & 08-03-1999 & 5 & 3,469 & 74.9 & 7.50 \\
\hline EB & 03-07-2000 & 4 & 2,407 & 77.6 & 3.90 \\
\hline
\end{tabular}


Table 28. Concentration of selenium in whole body tissue composite samples of common carp collected from the Green River near Jensen, Utah, 1996-2000-Continued

\begin{tabular}{|c|c|c|c|c|c|}
\hline $\begin{array}{l}\text { Abbreivated } \\
\text { site name }\end{array}$ & Date & $\begin{array}{l}\text { Number of } \\
\text { fish }\end{array}$ & $\begin{array}{l}\text { Total weight } \\
\text { (grams) }\end{array}$ & $\begin{array}{l}\text { Percent } \\
\text { moisture }\end{array}$ & $\begin{array}{l}\text { Selenium } \\
(\mu \mathrm{g} / \mathrm{g})\end{array}$ \\
\hline EB & $03-07-2000$ & 5 & 4,336 & 78.2 & 4.48 \\
\hline $\mathrm{EB}$ & 03-07-2000 & 5 & 5,810 & 77.6 & 5.43 \\
\hline $\mathrm{EB}$ & $08-08-2000$ & 4 & 2,415 & 75.3 & 10.4 \\
\hline $\mathrm{EB}$ & 08-08-2000 & 4 & 3,460 & 75.4 & 7.76 \\
\hline EB & $08-08-2000$ & 3 & 3,758 & 71.7 & 7.84 \\
\hline HA & 08-07-1997 & 5 & 6,331 & 71.5 & 2.57 \\
\hline HA & 08-07-1997 & 5 & 2,567 & 76.0 & 11.9 \\
\hline HA & 08-07-1997 & 5 & 1,457 & 78.8 & 11.2 \\
\hline HA & 08-12-1998 & 3 & 4,112 & 74.8 & 4.57 \\
\hline HA & 08-12-1998 & 4 & 4,150 & 75.8 & 3.22 \\
\hline HA & 08-12-1998 & 3 & 1,943 & 72.8 & 2.68 \\
\hline HA & 08-12-1998 & 4 & 526 & 79.6 & 8.01 \\
\hline HA & 03-16-1999 & 4 & 8,391 & 76.7 & 3.89 \\
\hline HA & 03-16-1999 & 5 & 7,573 & 75.8 & 4.86 \\
\hline HA & 03-16-1999 & 5 & 4,196 & 77.8 & 4.48 \\
\hline HA & 08-04-1999 & 5 & 2,987 & 74.4 & 3.73 \\
\hline HA & 08-04-1999 & 5 & 4,673 & 75.2 & 2.72 \\
\hline HA & 08-04-1999 & 5 & 6,189 & 71.6 & 5.10 \\
\hline HA & $03-14-2000$ & 5 & 2,905 & 79.1 & 2.49 \\
\hline HA & $03-14-2000$ & 5 & 6,715 & 76.5 & 4.05 \\
\hline HA & $03-14-2000$ & 5 & 4,939 & 77.3 & 3.03 \\
\hline HA & 08-09-2000 & 3 & 1,521 & 73.5 & 8.52 \\
\hline HA & 08-09-2000 & 4 & 3,106 & 72.5 & 3.65 \\
\hline HA & 08-09-2000 & 3 & 3,618 & 67.8 & 3.09 \\
\hline $\mathrm{HB}$ & 08-07-1997 & 4 & 5,760 & 72.3 & 3.37 \\
\hline $\mathrm{HB}$ & 08-07-1997 & 5 & 4,270 & 74.2 & 7.58 \\
\hline $\mathrm{HB}$ & 08-07-1997 & 5 & 2,580 & 75.2 & 4.36 \\
\hline $\mathrm{HB}$ & 08-07-1997 & 5 & 1,005 & 78.5 & 8.92 \\
\hline $\mathrm{HB}$ & 08-12-1998 & 5 & 2,410 & 77.4 & 5.82 \\
\hline $\mathrm{HB}$ & 08-12-1998 & 5 & 3,688 & 75.2 & 5.77 \\
\hline $\mathrm{HB}$ & 08-12-1998 & 5 & 4,225 & 75.0 & 2.28 \\
\hline $\mathrm{HB}$ & 03-16-1999 & 5 & 4,288 & 76.5 & 6.79 \\
\hline $\mathrm{HB}$ & 03-16-1999 & 5 & 2,760 & 78.0 & 2.81 \\
\hline $\mathrm{HB}$ & 03-16-1999 & 5 & 1,991 & 78.9 & 9.47 \\
\hline $\mathrm{HB}$ & 08-04-1999 & 3 & 3,959 & 74.1 & 4.23 \\
\hline $\mathrm{HB}$ & 08-04-1999 & 5 & 4,148 & 73.4 & 5.21 \\
\hline $\mathrm{HB}$ & 08-04-1999 & 4 & 1,462 & 76.4 & 3.41 \\
\hline $\mathrm{HB}$ & $03-08-2000$ & 5 & 6,631 & 75.8 & 3.76 \\
\hline $\mathrm{HB}$ & $03-08-2000$ & 5 & 4,405 & 75.6 & 2.71 \\
\hline $\mathrm{HB}$ & 03-08-2000 & 5 & 3,216 & 76.6 & 3.29 \\
\hline $\mathrm{HB}$ & 08-09-2000 & 5 & 2,522 & 75.2 & 9.89 \\
\hline HB & 08-09-2000 & 5 & 5,150 & 71.3 & 2.72 \\
\hline HB & 08-09-2000 & 3 & 5,807 & 69.5 & 4.02 \\
\hline LB & 08-06-1997 & 5 & 6,972 & 71.5 & 2.77 \\
\hline LB & 080-6-1997 & 5 & 6,685 & 70.8 & 6.16 \\
\hline LB & 08-06-1997 & 5 & 4,804 & 72.1 & 3.09 \\
\hline LB & 08-13-1998 & 5 & 3,678 & 76.6 & 2.17 \\
\hline
\end{tabular}


Table 28. Concentration of selenium in whole body tissue composite samples of common carp collected from the Green River near Jensen, Utah, 1996-2000-Continued

\begin{tabular}{|c|c|c|c|c|c|}
\hline $\begin{array}{l}\text { Abbreivated } \\
\text { site name }\end{array}$ & Date & $\begin{array}{l}\text { Number of } \\
\text { fish }\end{array}$ & $\begin{array}{l}\text { Total weight } \\
\text { (grams) }\end{array}$ & $\begin{array}{l}\text { Percent } \\
\text { moisture }\end{array}$ & $\begin{array}{c}\text { Selenium } \\
(\mu \mathrm{g} / \mathrm{g})\end{array}$ \\
\hline LB & 08-13-1998 & 5 & 3,219 & 77.8 & 1.69 \\
\hline LB & 08-13-1998 & 5 & 2,248 & 76.5 & 6.81 \\
\hline LB & 08-13-1998 & 3 & 493 & 80.2 & 3.70 \\
\hline LB & 03-18-1999 & 5 & 10,197 & 73.9 & 5.64 \\
\hline LB & 03-18-1999 & 5 & 3,489 & 78.7 & 5.72 \\
\hline LB & 03-18-1999 & 5 & 6,071 & 77.3 & 4.50 \\
\hline LB & 08-05-1999 & 5 & 2,995 & 75.8 & 1.52 \\
\hline LB & 08-05-1999 & 5 & 4,851 & 74.9 & 1.52 \\
\hline LB & 08-05-1999 & 5 & 6,114 & 73.4 & 1.62 \\
\hline LB & 03-14-2000 & 5 & 3,836 & 76.9 & 2.11 \\
\hline LB & 03-14-2000 & 5 & 6,633 & 75.4 & 2.84 \\
\hline LB & 03-14-2000 & 5 & 4,738 & 74.9 & 1.49 \\
\hline LB & 08-09-2000 & 5 & 3,540 & 77.9 & 1.80 \\
\hline LB & 08-09-2000 & 4 & 6,165 & 71.1 & 4.51 \\
\hline LB & 08-09-2000 & 5 & 5,389 & 70.2 & 4.30 \\
\hline SA & 08-01-1996 & 5 & 3,541 & 74.1 & 34.6 \\
\hline SA & 08-01-1996 & 5 & 3,892 & 72.9 & 11.6 \\
\hline SA & 08-01-1996 & 5 & 6,060 & 71.1 & 12.1 \\
\hline SA & 08-05-1997 & 5 & 6,635 & 74.8 & 17.8 \\
\hline SA & 08-05-1997 & 5 & 2,857 & 77.5 & 13.3 \\
\hline SA & 08-05-1997 & 5 & 4,290 & 74.1 & 24.3 \\
\hline SA & 08-11-1998 & 3 & 909 & 77.4 & 4.76 \\
\hline SA & 08-11-1998 & 3 & 3,195 & 77.0 & 12.2 \\
\hline SA & 08-11-1998 & 4 & 2,451 & 77.6 & 15.0 \\
\hline SA & 03-17-1999 & 5 & 2,830 & 78.1 & 20.4 \\
\hline SA & 03-17-1999 & 5 & 3,853 & 76.5 & 20.6 \\
\hline SA & 03-17-1999 & 5 & 6,041 & 76.7 & 12.4 \\
\hline SA & 08-03-1999 & 3 & 3,593 & 74.4 & 5.69 \\
\hline SA & 08-03-1999 & 4 & 3,551 & 72.5 & 2.64 \\
\hline SA & 08-03-1999 & 3 & 2,107 & 75.6 & 9.87 \\
\hline SA & 03-08-2000 & 5 & 6,789 & 78.1 & 18.8 \\
\hline SA & 03-08-2000 & 5 & 2,665 & 80.2 & 9.26 \\
\hline SA & 03-08-2000 & 5 & 4,402 & 77.2 & 15.3 \\
\hline SA & $08-08-2000$ & 5 & 3,164 & 74.9 & 12.1 \\
\hline SA & 08-08-2000 & 5 & 6,954 & 75.5 & 17.8 \\
\hline SA & 08-08-2000 & 5 & 4,475 & 73.7 & 14.9 \\
\hline SB & 07-31-1996 & 5 & 4,429 & 73.1 & 3.4 \\
\hline SB & 07-31-1996 & 5 & 4,678 & 70.1 & 4.8 \\
\hline SB & 07-31-1996 & 5 & 5,734 & 71.2 & 2.7 \\
\hline SB & 08-06-1997 & 5 & 5,168 & 73.9 & 2.97 \\
\hline SB & 08-06-1997 & 5 & 9,249 & 70.1 & 3.43 \\
\hline SB & 08-06-1997 & 5 & 6,640 & 71.9 & 4.43 \\
\hline SB & 08-13-1998 & 5 & 6,721 & 73.1 & 3.57 \\
\hline SB & 08-13-1998 & 5 & 5,286 & 74.7 & 3.08 \\
\hline SB & 08-13-1998 & 5 & 3,139 & 77.3 & 6.02 \\
\hline SB & 03-18-1999 & 5 & 8,394 & 77.2 & 4.73 \\
\hline SB & 03-18-1999 & 4 & 3,154 & 76.6 & 3.64 \\
\hline
\end{tabular}


Table 28. Concentration of selenium in whole body tissue composite samples of common carp collected from the Green River near Jensen, Utah, 1996-2000-Continued

\begin{tabular}{lccccc}
\hline $\begin{array}{l}\text { Abbreivated } \\
\text { site name }\end{array}$ & Date & $\begin{array}{c}\text { Number of } \\
\text { fish }\end{array}$ & $\begin{array}{c}\text { Total weight } \\
\text { (grams) }\end{array}$ & $\begin{array}{c}\text { Percent } \\
\text { moisture }\end{array}$ & $\begin{array}{c}\text { Selenium } \\
(\boldsymbol{\mu g} / \mathbf{g})\end{array}$ \\
\hline SB & $03-18-1999$ & 5 & 6,019 & 76.0 & 4.64 \\
SB & $08-05-1999$ & 5 & 3,834 & 75.4 & 1.68 \\
SB & $08-05-1999$ & 5 & 5,468 & 75.0 & 2.83 \\
SB & $08-05-1999$ & 5 & 4,314 & 74.1 & 1.52 \\
SB & $03-14-2000$ & 5 & 3,755 & 78.7 & 2.36 \\
SB & $03-14-2000$ & 5 & 5,405 & 75.4 & 1.93 \\
SB & $03-14-2000$ & 5 & 6,965 & 76.2 & 3.45 \\
SB & $08-09-2000$ & 4 & 3,354 & 73.1 & 2.84 \\
SB & $08-09-2000$ & 3 & 2,912 & 75.0 & 2.08 \\
SB & $08-09-2000$ & 3 & 3,833 & 70.3 & 5.25 \\
SLOC & $03-17-1999$ & 4 & 2,968 & 76.6 & 23.3 \\
SM & $03-15-2000$ & 3 & 2,752 & 78.6 & 3.43 \\
SM & $03-15-2000$ & 4 & 3,587 & 70.8 & 5.03 \\
SM & $03-15-2000$ & 4 & 3,995 & 72.3 & 1.84 \\
SM & $08-10-2000$ & 4 & 3,430 & 70.1 & 6.82 \\
SM & $08-10-2000$ & 4 & 4,098 & 76.8 & 7.14 \\
SM & $08-10-2000$ & 5 & 7,330 & 78.8 & 8.15 \\
SM & $08-10-2000$ & 3 & 3,228 & & 2.23 \\
\hline
\end{tabular}


Table 29. Selenium concentration in crayfish and fish samples collected from Green River backwaters near Stewart Lake Waterfowl Management Area near Jensen, Utah, 1997-2000

[Data from U.S. Fish and Wildlife Service; $\mu \mathrm{g} / \mathrm{g}$, micrograms per gram, dry weight; —, not determined; <, less than]

\begin{tabular}{|c|c|c|c|c|c|c|}
\hline Abbreviated site name & Date & Species & $\begin{array}{l}\text { Number } \\
\text { of fish }\end{array}$ & $\begin{array}{l}\text { Total weight } \\
\text { (grams) }\end{array}$ & $\begin{array}{l}\text { Percent } \\
\text { moisture }\end{array}$ & $\begin{array}{l}\text { Selenium } \\
(\mu \mathrm{g} / \mathrm{g})\end{array}$ \\
\hline \multirow[t]{10}{*}{ GR1 } & 07-29-1997 & Common Carp & - & 32 & 81.0 & 3.71 \\
\hline & 08-26-1997 & Mixed Fish & - & 41 & 78.9 & 4.06 \\
\hline & 07-14-1998 & Green Sunfish & - & 13 & 79.3 & 2.37 \\
\hline & 06-17-1998 & Shiners & - & 19 & 75.0 & 3.97 \\
\hline & 07-14-1998 & Shiners & - & 79 & 76.4 & 2.88 \\
\hline & 07-27-1999 & Common Carp & 45 & 56 & 81.8 & 4.49 \\
\hline & 07-27-1999 & Shiners & 16 & 23 & 77.5 & 4.67 \\
\hline & 03-06-2000 & Fathead Minnow & 15 & 33 & 80.0 & 8.09 \\
\hline & 03-06-2000 & Shiners & 20 & 29 & 74.7 & 5.10 \\
\hline & $05-17-2000$ & Mixed Fish & 26 & 47 & 84.2 & 7.16 \\
\hline \multirow[t]{17}{*}{ GR2 } & 07-29-1997 & Black Bullhead & 5 & 306 & 78.3 & 6.66 \\
\hline & 07-29-1997 & Common Carp & - & 90 & 80.6 & 3.94 \\
\hline & 08-26-1997 & Crayfish & 6 & 27 & 74.0 & .90 \\
\hline & 07-29-1997 & Fathead Minnow & - & 31 & 82.3 & 6.27 \\
\hline & 08-26-1997 & Mixed Fish & - & 39 & 79.4 & 4.55 \\
\hline & 07-14-1998 & Black Bullhead & - & 47 & 78.4 & 4.50 \\
\hline & 07-14-1998 & Black Bullhead & - & 90 & 80.1 & 9.56 \\
\hline & 07-14-1998 & Crappie sp. & 1 & 30 & 72.8 & 2.63 \\
\hline & 07-14-1998 & Crayfish & - & 298 & 78.8 & 4.28 \\
\hline & 07-14-1998 & Northern Pike & - & 229 & 78.1 & 7.82 \\
\hline & 07-09-1999 & Black Bullhead & 3 & 112 & 79.5 & 5.14 \\
\hline & 07-09-1999 & Common Carp & 21 & 40 & 77.6 & 5.21 \\
\hline & 07-28-1999 & Common Carp & 25 & 73 & 80.4 & 6.36 \\
\hline & 07-09-1999 & Crayfish & 1 & 12 & 78.4 & 2.64 \\
\hline & 07-09-1999 & Fathead Minnow & 9 & 20 & 77.6 & 8.80 \\
\hline & 04-11-2000 & Shiners & 13 & 31 & 77.7 & 12.5 \\
\hline & $05-17-2000$ & Fathead Minnow & 34 & 38 & 83.9 & 9.04 \\
\hline GR2 -SL2 & 07-28-1999 & Common Carp & 25 & 85 & 81.0 & 19.3 \\
\hline \multirow[t]{16}{*}{ GR3 } & 07-29-1997 & Common Carp & - & 62 & 77.8 & 4.69 \\
\hline & 08-26-1997 & Common Carp & - & 49 & 79.5 & 3.74 \\
\hline & 07-29-1997 & Green Sunfish & - & 63 & 78.4 & 4.18 \\
\hline & 08-26-1997 & Green Sunfish & - & 45 & 77.4 & 3.27 \\
\hline & 06-17-1998 & Common Carp & - & 10 & 87.3 & 12.1 \\
\hline & 08-10-1998 & Common Carp & - & 53 & 78.7 & 2.73 \\
\hline & 06-17-1998 & Fathead Minnow & - & 59 & 77.7 & 2.34 \\
\hline & 08-10-1998 & Fathead Minnow & - & 43 & 77.5 & 3.04 \\
\hline & 07-14-1998 & Mixed Fish & - & 61 & 79.2 & 3.08 \\
\hline & 06-17-1998 & Shiners & - & 41 & 74.9 & 4.00 \\
\hline & 08-10-1998 & Shiners & - & 10 & 72.5 & 2.26 \\
\hline & 07-21-1999 & Common Carp & 50 & 70 & 79.6 & 7.12 \\
\hline & 07-21-1999 & Shiners & 50 & 66 & 78.0 & 5.65 \\
\hline & 03-06-2000 & Fathead Minnow & 10 & 20 & 78.3 & 16.0 \\
\hline & 03-06-2000 & Shiners & 20 & 26 & 76.7 & 10.8 \\
\hline & $05-16-2000$ & Fathead Minnow & 20 & 32 & 79.2 & 12.5 \\
\hline
\end{tabular}


Table 29. Selenium concentration in crayfish and fish samples collected from Green River backwaters near Stewart Lake Waterfowl Management Area near Jensen, Utah, 1997-2000-Continued

\begin{tabular}{|c|c|c|c|c|c|c|}
\hline Abbreviated site name & Date & Species & $\begin{array}{l}\text { Number } \\
\text { of fish }\end{array}$ & $\begin{array}{l}\text { Total weight } \\
\text { (grams) }\end{array}$ & $\begin{array}{l}\text { Percent } \\
\text { moisture }\end{array}$ & $\begin{array}{c}\text { Selenium } \\
(\mu \mathrm{g} / \mathrm{g})\end{array}$ \\
\hline \multirow[t]{3}{*}{ GR3-Continued } & $05-16-2000$ & Shiners & 8 & 11 & 76.9 & 6.37 \\
\hline & 08-10-2000 & Common Carp & 20 & 27 & 79.9 & 13.0 \\
\hline & 08-10-2000 & Fathead Minnow & 25 & 41 & 75.0 & 12.2 \\
\hline \multirow[t]{9}{*}{ GR4 } & 07-29-1997 & Common Carp & - & 29 & 81.3 & 7.45 \\
\hline & 07-29-1997 & Shiners & - & 66 & 80.8 & 2.98 \\
\hline & 08-26-1997 & Shiners & - & 85 & 76.2 & 6.90 \\
\hline & 06-17-1998 & Fathead Minnow & - & 34 & 81.1 & 3.81 \\
\hline & 06-17-1998 & Green Sunfish & - & 23 & 78.7 & 14.0 \\
\hline & 07-21-1999 & Mixed Fish & 11 & 4 & 80.9 & 10.9 \\
\hline & $05-17-2000$ & Fathead Minnow & 20 & 25 & 77.9 & 23.4 \\
\hline & $05-17-2000$ & Green Sunfish & 11 & 29 & 84.2 & 9.00 \\
\hline & $05-17-2000$ & Shiners & 20 & 33 & 79.9 & 10.9 \\
\hline \multirow[t]{10}{*}{ GR5 } & 07-29-1997 & Common Carp & - & 48 & 77.0 & 4.48 \\
\hline & 07-29-1997 & Green Sunfish & - & 40 & 75.9 & 4.98 \\
\hline & 07-27-1999 & Common Carp & 35 & 50 & 81.9 & 4.91 \\
\hline & 07-27-1999 & Shiners & 9 & 16 & 75.1 & 4.23 \\
\hline & 03-07-2000 & Fathead Minnow & 10 & 10 & 76.7 & 9.71 \\
\hline & 03-07-2000 & Shiners & 20 & 12 & 77.4 & 5.95 \\
\hline & $05-17-2000$ & Fathead Minnow & 20 & 34 & 80.1 & 23.4 \\
\hline & $05-17-2000$ & Green Sunfish & 8 & 23 & 81.9 & 22.0 \\
\hline & 08-10-2000 & Common Carp & 11 & 31 & 82.6 & 13.0 \\
\hline & 08-10-2000 & Green Sunfish & 13 & 27 & 80.4 & 8.38 \\
\hline \multirow[t]{15}{*}{ GR6 } & 08-06-1997 & Common Carp & - & 57 & 79.4 & 6.32 \\
\hline & 08-26-1997 & Common Carp & - & 64 & 78.9 & 4.31 \\
\hline & 08-26-1997 & Common Carp & 1 & 308 & 78.0 & 13.7 \\
\hline & 08-06-1997 & Fathead Minnow & - & 21 & 74.3 & 3.33 \\
\hline & 08-26-1997 & Green Sunfish & - & 58 & 75.0 & 5.00 \\
\hline & 08-26-1997 & Green Sunfish & - & 45 & 74.7 & 4.92 \\
\hline & 08-11-1998 & Common Carp & - & 11 & 77.1 & 8.18 \\
\hline & 06-17-1998 & Mixed Fish & - & 59 & 79.1 & 6.12 \\
\hline & 08-11-1998 & Shiners & - & 47 & 75.9 & 3.92 \\
\hline & 07-27-1999 & Common Carp & 33 & 43 & 83.0 & 7.28 \\
\hline & 07-27-1999 & Shiners & 5 & 6 & 76.7 & 4.60 \\
\hline & 03-07-2000 & Fathead Minnow & 20 & 25 & 79.6 & 20.8 \\
\hline & 03-07-2000 & Shiners & 20 & 32 & 82.5 & 6.50 \\
\hline & 08-10-2000 & Mixed Fish & 9 & 19 & 78.2 & 9.31 \\
\hline & $08-10-2000$ & Green Sunfish & 4 & 11 & 84.4 & 7.34 \\
\hline SLOC & 07-21-1999 & Mixed Fish & 14 & 17 & 77.6 & 9.37 \\
\hline \multirow[t]{4}{*}{$\mathrm{J} 1 \mathrm{~B}$} & $06-28-2000$ & Common Carp & 13 & 17 & 82.0 & 5.05 \\
\hline & $06-28-2000$ & Shiners & 30 & 36 & 75.2 & 7.87 \\
\hline & 08-10-2000 & Common Carp & 4 & 9 & 80.5 & 15.0 \\
\hline & 08-10-2000 & Shiners & 10 & 11 & 77.2 & 13.3 \\
\hline
\end{tabular}


Table 30. Concentration of selenium in common carp samples collected from Stewart Lake near Jensen, Utah, 1991-2000

[Data from U.S. Fish and Wildlife Service; $\mu \mathrm{g} / \mathrm{g}$, micrograms per gram, dry weight; —, not recorded]

\begin{tabular}{|c|c|c|c|c|c|}
\hline $\begin{array}{l}\text { Abbreviated } \\
\text { site name }\end{array}$ & Date & Number of Fish & $\begin{array}{l}\text { Total weight } \\
\text { (grams) }\end{array}$ & $\begin{array}{l}\text { Percent } \\
\text { moisture }\end{array}$ & $\begin{array}{c}\text { Selenium } \\
(\mu \mathrm{g} / \mathrm{g})\end{array}$ \\
\hline \multirow[t]{47}{*}{ SLN } & 08-08-1991 & - & 2,579 & 76.3 & 53.0 \\
\hline & 08-08-1991 & - & 2,677 & 77.3 & 47.0 \\
\hline & 05-17-1995 & 5 & 4,607 & 74.5 & 24.7 \\
\hline & 07-13-1995 & 5 & 893 & 78.4 & 31.5 \\
\hline & 07-13-1995 & 5 & 5,042 & 75.2 & 14.7 \\
\hline & 11/28-1995 & 3 & 1,710 & 75.1 & 40.7 \\
\hline & 11/28-1995 & 3 & 2,657 & 74.0 & 37.3 \\
\hline & 04-23-1996 & 5 & 3,692 & 76.7 & 34.5 \\
\hline & 04-23-1996 & 3 & 6,290 & 76.9 & 34.2 \\
\hline & 04-23-1996 & 5 & 1,080 & 78.5 & 19.8 \\
\hline & 10-24-1996 & 5 & 830 & 76.5 & 22.7 \\
\hline & 04-15-1997 & 3 & 989 & 79.7 & 37.0 \\
\hline & 04-15-1997 & 4 & 1,127 & 78.2 & 31.7 \\
\hline & 04-15-1997 & 5 & 868 & 77.9 & 29.3 \\
\hline & 05-28-1997 & 5 & 1,272 & 79.3 & 30.9 \\
\hline & 05-28-1997 & 5 & 5,385 & 76.0 & 27.3 \\
\hline & 05-28-1997 & 5 & 4,702 & 78.0 & 23.3 \\
\hline & 06-10-1997 & 5 & 5,448 & 77.8 & 29.0 \\
\hline & 06-10-1997 & 5 & 3,678 & 77.7 & 29.0 \\
\hline & 06-10-1997 & 5 & 1,320 & 79.0 & 26.6 \\
\hline & 07-08-1997 & 5 & 4,165 & 76.9 & 32.1 \\
\hline & 07-08-1997 & 5 & 2,676 & 75.3 & 26.0 \\
\hline & 07-08-1997 & 5 & 987 & 78.6 & 24.2 \\
\hline & 08-27-1997 & 5 & 1,199 & 77.2 & 27.1 \\
\hline & 08-27-1997 & 5 & 4,204 & 78.2 & 25.7 \\
\hline & 08-27-1997 & 5 & 2,649 & 76.2 & 21.8 \\
\hline & 04-22-1998 & 5 & 500 & 83.7 & 22.5 \\
\hline & 04-22-1998 & 5 & 2,509 & 78.5 & 19.2 \\
\hline & 04-22-1998 & 3 & 3,013 & 79.3 & 17.5 \\
\hline & 04-22-1998 & 5 & 3,360 & 79.0 & 15.7 \\
\hline & 05-20-1998 & 5 & 724 & 79.6 & 29.2 \\
\hline & 05-20-1998 & 3 & 2,697 & 79.1 & 10.4 \\
\hline & 06-02-1998 & 5 & 838 & 81.2 & 32.9 \\
\hline & 06-02-1998 & 3 & 2,424 & 79.0 & 31.7 \\
\hline & 06-02-1998 & 5 & 1,829 & 78.9 & 19.7 \\
\hline & 06-16-1998 & 5 & 3,081 & 79.5 & 27.8 \\
\hline & 06-16-1998 & 5 & 4,343 & 76.8 & 25.4 \\
\hline & 06-16-1998 & 3 & 303 & 79.4 & 24.7 \\
\hline & 06-16-1998 & 2 & 47 & 80.8 & 21.2 \\
\hline & 08-27-1998 & 5 & 2,945 & 80.2 & 30.0 \\
\hline & 06-03-1999 & 4 & 837 & 80.5 & 24.0 \\
\hline & 06-03-1999 & 5 & 3,853 & 78.1 & 19.8 \\
\hline & 06-03-1999 & 4 & 4,423 & 76.6 & 14.6 \\
\hline & 06-22-1999 & 5 & 1,880 & 79.2 & 19.6 \\
\hline & 06-22-1999 & 5 & 1,299 & 78.9 & 19.1 \\
\hline & 06-22-1999 & 5 & 5,702 & 75.1 & 10.7 \\
\hline & 07-08-1999 & 4 & 56 & 82.2 & 31.7 \\
\hline
\end{tabular}


Table 30. Concentration of selenium in common carp samples collected from Stewart Lake near Jensen, Utah, 1991-2000—Continued

\begin{tabular}{|c|c|c|c|c|c|}
\hline $\begin{array}{l}\text { Abbreviated } \\
\text { site name }\end{array}$ & Date & Number of Fish & $\begin{array}{l}\text { Total weight } \\
\text { (grams) }\end{array}$ & $\begin{array}{l}\text { Percent } \\
\text { moisture }\end{array}$ & $\begin{array}{c}\text { Selenium } \\
(\mu \mathrm{g} / \mathrm{g})\end{array}$ \\
\hline \multirow[t]{10}{*}{ SLN-Continued } & 07-08-1999 & 5 & 1,781 & 78.8 & 23.9 \\
\hline & 07-08-1999 & 5 & 1,367 & 79.7 & 19.1 \\
\hline & 07-08-1999 & 5 & 3,270 & 77.7 & 16.5 \\
\hline & 07-20-1999 & 5 & 1,810 & 80.1 & 23.8 \\
\hline & 07-20-1999 & 5 & 3,352 & 75.9 & 16.5 \\
\hline & $05-31-2000$ & 3 & 3,650 & 77.9 & 23.9 \\
\hline & $05-31-2000$ & 3 & 1,060 & 79.2 & 19.1 \\
\hline & 06-14-2000 & 3 & 2,449 & 76.4 & 21.1 \\
\hline & 06-14-2000 & 3 & 4,217 & 76.3 & 26.0 \\
\hline & $06-27-2000$ & 4 & 3,360 & 73.6 & 20.3 \\
\hline \multirow[t]{36}{*}{ SLO } & 08-08-1991 & - & 2,400 & 76.0 & 38.0 \\
\hline & 08-09-1991 & - & 2,655 & 76.6 & 35.0 \\
\hline & 05-17-1995 & 5 & 2,725 & 74.9 & 27.2 \\
\hline & $07-12-1995$ & 5 & 933 & 79.2 & 19.7 \\
\hline & 07-12-1995 & 5 & 887 & 79.1 & 10.3 \\
\hline & 04-23-1996 & 3 & 2,507 & 76.0 & 17.3 \\
\hline & $10-24-1996$ & 5 & 313 & 74.7 & 28.0 \\
\hline & 10-24-1996 & 4 & 344 & 75.8 & 24.7 \\
\hline & 10-24-1996 & 4 & 944 & 76.8 & 23.2 \\
\hline & 04-15-1997 & 2 & 134 & 82.0 & 29.1 \\
\hline & 04-15-1997 & 5 & 376 & 79.9 & 29.0 \\
\hline & 04-15-1997 & 5 & 620 & 79.5 & 23.6 \\
\hline & 05-28-1997 & 5 & 1,173 & 78.4 & 36.2 \\
\hline & 05-28-1997 & 5 & 4,510 & 79.3 & 15.2 \\
\hline & 06-10-1997 & 4 & 785 & 78.5 & 29.0 \\
\hline & 06-10-1997 & 5 & 4,075 & 78.4 & 26.2 \\
\hline & 06-10-1997 & 2 & 2,465 & 78.4 & 10.5 \\
\hline & 07-08-1997 & 5 & 3,185 & 77.8 & 23.0 \\
\hline & 07-08-1997 & 5 & 4,583 & 78.2 & 23.0 \\
\hline & 07-08-1997 & 5 & 969 & 78.2 & 21.2 \\
\hline & 08-27-1997 & 2 & 1,672 & 75.9 & 22.0 \\
\hline & 08-27-1997 & 2 & 1,092 & 76.1 & 11.2 \\
\hline & 04-21-1998 & 5 & 3,219 & 77.7 & 19.2 \\
\hline & 04-21-1998 & 5 & 3,933 & 78.1 & 18.6 \\
\hline & 04-21-1998 & 5 & 1,081 & 81.2 & 15.9 \\
\hline & 05-20-1998 & 5 & 955 & 80.1 & 15.9 \\
\hline & 05-20-1998 & 5 & 2,861 & 77.7 & 13.0 \\
\hline & 05-20-1998 & 5 & 5,150 & 77.3 & 7.82 \\
\hline & 06-02-1998 & 5 & 3,928 & 79.0 & 9.48 \\
\hline & 06-02-1998 & 5 & 1,161 & 82.9 & 7.27 \\
\hline & 06-02-1998 & 5 & 2,473 & 79.2 & 2.12 \\
\hline & 06-16-1998 & 4 & 3,521 & 75.0 & 20.0 \\
\hline & 06-16-1998 & 5 & 922 & 79.1 & 15.2 \\
\hline & 06-16-1998 & 5 & 1,710 & 78.2 & 8.02 \\
\hline & 07-15-1998 & 5 & 1,072 & 82.8 & 21.5 \\
\hline & 07-15-1998 & 5 & 3,809 & 77.6 & 20.0 \\
\hline
\end{tabular}


Table 30. Concentration of selenium in common carp samples collected from Stewart Lake near Jensen, Utah, 1991-2000—Continued

\begin{tabular}{|c|c|c|c|c|c|}
\hline $\begin{array}{l}\text { Abbreviated } \\
\text { site name }\end{array}$ & Date & Number of Fish & $\begin{array}{l}\text { Total weight } \\
\text { (grams) }\end{array}$ & $\begin{array}{l}\text { Percent } \\
\text { moisture }\end{array}$ & $\begin{array}{l}\text { Selenium } \\
(\mu \mathrm{g} / \mathrm{g})\end{array}$ \\
\hline \multirow[t]{26}{*}{ SLO-Continued } & 08-27-1998 & 3 & 2,436 & 81.3 & 23.5 \\
\hline & 08-27-1998 & 3 & 1,425 & 81.3 & 23.3 \\
\hline & 08-27-1998 & 3 & 1,939 & 86.9 & 19.0 \\
\hline & 05-18-1999 & 4 & 2,211 & 79.2 & 16.8 \\
\hline & 05-18-1999 & 4 & 3,872 & 75.5 & 7.22 \\
\hline & 05-18-1999 & 5 & 6,629 & 73.4 & 5.94 \\
\hline & 06-02-1999 & 4 & 3,223 & 79.0 & 22.8 \\
\hline & 06-02-1999 & 2 & 374 & 80.2 & 3.31 \\
\hline & 06-22-1999 & 5 & 1,542 & 78.7 & 12.6 \\
\hline & 06-22-1999 & 5 & 4,419 & 72.8 & 9.29 \\
\hline & 06-22-1999 & 4 & 5,266 & 77.5 & 8.70 \\
\hline & 07-07-1999 & 4 & 2,979 & 76.1 & 20.7 \\
\hline & 07-07-1999 & 3 & 984 & 78.8 & 19.0 \\
\hline & 07-07-1999 & 3 & 4,160 & 70.5 & 7.36 \\
\hline & 07-20-1999 & 4 & 1,708 & 78.8 & 18.1 \\
\hline & 07-20-1999 & 3 & 811 & 80.4 & 17.0 \\
\hline & 07-20-1999 & 4 & 4,284 & 75.4 & 10.0 \\
\hline & $05-17-2000$ & 3 & 941 & 81.5 & 15.9 \\
\hline & 06-01-2000 & 5 & 2,445 & 79.1 & 14.0 \\
\hline & $06-13-2000$ & 3 & 2,421 & 76.9 & 10.3 \\
\hline & $06-13-2000$ & 3 & 3,605 & 76.1 & 12.8 \\
\hline & $06-28-2000$ & 3 & 1,878 & 75.5 & 13.1 \\
\hline & $06-28-2000$ & 3 & 3,855 & 74.7 & 22.7 \\
\hline & $07-10-2000$ & 5 & 3,587 & 74.1 & 17.9 \\
\hline & $07-10-2000$ & 3 & 1,957 & 73.6 & 14.1 \\
\hline & $07-10-2000$ & 3 & 2,917 & 73.7 & 13.6 \\
\hline \multirow[t]{20}{*}{ SLJ1 } & 10-24-1996 & 4 & 836 & 78.0 & 18.7 \\
\hline & 04-15-1997 & 2 & 1,488 & 76.9 & 29.3 \\
\hline & 04-15-1997 & 3 & 626 & 78.1 & 20.6 \\
\hline & 04-15-1997 & 5 & 882 & 80.3 & 17.8 \\
\hline & 04-15-1997 & 5 & 276 & 79.5 & 24.4 \\
\hline & 04-15-1997 & 5 & 540 & 79.8 & 22.6 \\
\hline & 06-10-1997 & 3 & 642 & 78.1 & 24.7 \\
\hline & 06-10-1997 & 5 & 1,082 & 78.7 & 23.2 \\
\hline & 06-10-1997 & 3 & 1,839 & 76.1 & 17.6 \\
\hline & 07-08-1997 & 5 & 1,025 & 77.3 & 20.5 \\
\hline & 07-08-1997 & 2 & 2,130 & 77.8 & 19.4 \\
\hline & 07-08-1997 & 5 & 1,153 & 77.5 & 15.1 \\
\hline & 08-27-1997 & 5 & 1,400 & 78.3 & 16.9 \\
\hline & 08-27-1997 & 5 & 373 & 78.7 & 16.2 \\
\hline & 05-19-1998 & 5 & 1,037 & 81.5 & 10.5 \\
\hline & 05-19-1998 & 5 & 3,026 & 80.0 & 7.82 \\
\hline & 06-02-1998 & 5 & 1,048 & 79.6 & 16.2 \\
\hline & 06-02-1998 & 5 & 4,681 & 78.1 & 14.7 \\
\hline & 06-02-1998 & 5 & 3,265 & 76.2 & 8.60 \\
\hline & 06-16-1998 & 5 & 4,471 & 79.8 & 17.1 \\
\hline SLJ1-Continued & 06-16-1998 & 5 & 2,028 & 76.7 & 16.9 \\
\hline
\end{tabular}


Table 30. Concentration of selenium in common carp samples collected from Stewart Lake near Jensen, Utah, 1991-2000—Continued

\begin{tabular}{cccccc}
\hline $\begin{array}{c}\text { Abbreviated } \\
\text { site name }\end{array}$ & Date & Number of Fish & $\begin{array}{c}\text { Total weight } \\
\text { (grams) }\end{array}$ & $\begin{array}{c}\text { Percent } \\
\text { moisture }\end{array}$ & $\begin{array}{c}\text { Selenium } \\
(\mu \mathbf{g} / \mathbf{g})\end{array}$ \\
\hline & $06-16-1998$ & 5 & 970 & 78.2 & 13.8 \\
& $06-03-1999$ & 2 & 375 & 78.0 & 40.9 \\
& $06-22-1999$ & 4 & 4,420 & 76.7 & 12.3 \\
& $06-22-1999$ & 3 & 5,214 & 73.8 & 8.59 \\
& $06-22-1999$ & 5 & 3,662 & 77.2 & 3.95 \\
& $07-07-1999$ & 5 & 1,327 & 79.1 & 19.9 \\
& $07-07-1999$ & 5 & 5,855 & 74.5 & 17.3 \\
& $07-07-1999$ & 5 & 2,048 & 77.2 & 5.72 \\
& $05-31-2000$ & 3 & 3,950 & 76.9 & 22.8 \\
& $05-31-2000$ & 3 & 3,146 & 76.2 & 9.30 \\
& $05-31-2000$ & 4 & 2,753 & 77.1 & 8.77 \\
& $06-13-2000$ & 4 & 4,121 & 76.2 & 16.5 \\
& $06-13-2000$ & 3 & 1,540 & 77.4 & 12.1 \\
& $06-28-2000$ & 4 & 4,571 & 73.0 & 12.5 \\
SLM & $06-28-2000$ & 4 & 2,765 & 73.4 & 24.2 \\
& $03-18-1999$ & 5 & 3,462 & 79.3 & 22.4 \\
& $03-18-1999$ & 4 & 2,168 & 79.3 & 20.9 \\
& $03-18-1999$ & 4 & 1,274 & 79.4 & 22.6 \\
& $09-27-1999$ & 5 & 5,682 & 80.2 & 22.8 \\
& $09-27-1999$ & 5 & 3,724 & 77.8 & 31.5 \\
& $09-27-1999$ & 5 & 2,965 & 76.2 & 62.0 \\
\hline
\end{tabular}


Table 31. Concentration of selenium in samples of small fish collected from Stewart Lake Waterfowl Management Area near Jensen, Utah, 1994-2000 [Data from U.S. Fish and Wildlife Service; $\mu \mathrm{g} / \mathrm{g}$, micrograms per gram, dry weight; —, not determined]

\begin{tabular}{|c|c|c|c|c|c|c|}
\hline $\begin{array}{l}\text { Abbreviated } \\
\text { site } \\
\text { name }\end{array}$ & Species & Date & Number of fish & $\begin{array}{l}\text { Total weight } \\
\text { (grams) }\end{array}$ & $\begin{array}{l}\text { Percent } \\
\text { moisture }\end{array}$ & $\begin{array}{c}\text { Selenium } \\
(\mu \mathrm{g} / \mathrm{g})\end{array}$ \\
\hline \multirow[t]{11}{*}{ SLN } & Common Carp & $10-26-1994$ & 1 & - & 73.8 & 33.5 \\
\hline & Common Carp & 07-13-1998 & 1 & 46 & 84.1 & 18.2 \\
\hline & Common Carp & 07-08-1999 & 43 & 74 & 82.9 & 31.5 \\
\hline & Common Carp & 07-08-1999 & 46 & 73 & 83.8 & 29.8 \\
\hline & Common Carp & 07-20-1999 & 62 & 108 & 82.0 & 37.5 \\
\hline & Fathead Minnow & 05-20-1998 & - & 13 & 80.6 & 14.4 \\
\hline & Fathead Minnow & 07-13-1998 & - & 15 & 76.2 & 9.27 \\
\hline & Fathead Minnow & 05-18-1999 & 40 & 151 & 77.7 & 50.7 \\
\hline & Fathead Minnow & $05-31-2000$ & - & 11 & 78.9 & 33.2 \\
\hline & Green Sunfish & 06-01-2000 & - & 89 & 76.9 & 20.7 \\
\hline & Red Shiner & 07-12-1995 & - & 6 & 80.8 & 28.3 \\
\hline \multirow[t]{15}{*}{ SLO } & Common Carp & 07-08-1999 & 40 & 115 & 83.4 & 19.9 \\
\hline & Common Carp & 07-08-1999 & 40 & 87 & 82.9 & 18.5 \\
\hline & Common Carp & 07-20-1999 & 140 & 423 & 82.6 & 17.6 \\
\hline & Common Carp & $06-28-2000$ & 50 & 39 & 83.2 & 21.5 \\
\hline & Fathead Minnow & 05-18-1999 & 40 & 164 & 77.9 & 42.4 \\
\hline & Fathead Minnow & $05-17-2000$ & - & 82 & 83.1 & 25.1 \\
\hline & Fathead Minnow & 06-01-2000 & - & 14 & 75.9 & 20.6 \\
\hline & Green Sunfish & 05-18-1999 & 5 & 79 & 74.7 & 30.5 \\
\hline & Green Sunfish & 07-20-1999 & 100 & 35 & 80.5 & 13.0 \\
\hline & Green Sunfish & 06-01-2000 & - & 41 & 75.9 & 21.8 \\
\hline & Black Bullhead & 07-08-1997 & 100 & 6 & 89.6 & 2.45 \\
\hline & Larval fish & 06-01-2000 & - & 13 & 90.5 & 12.1 \\
\hline & Mixed fish & 06-22-1999 & 7 & 11 & 73.1 & 7.62 \\
\hline & Mixed fish & 07-09-1999 & 15 & 6 & 86.6 & 15.0 \\
\hline & Red Shiner & 04-15-1997 & 23 & 19 & 81.7 & 16.0 \\
\hline \multirow[t]{12}{*}{ SLI } & Common Carp & 07-21-1999 & 50 & 94 & 82.3 & 15.9 \\
\hline & Fathead Minnow & $05-31-2000$ & - & 42 & 74.9 & 19.9 \\
\hline & Green Sunfish & 07-21-1999 & 20 & 50 & 77.9 & 13.4 \\
\hline & Larval fish & 06-24-1997 & 151 & 5 & 84.8 & 3.60 \\
\hline & Larval fish & 06-25-1997 & 133 & 5 & 86.8 & 4.50 \\
\hline & Larval fish & 06-06-2000 & - & 4 & 93.6 & 8.33 \\
\hline & Larval fish & 06-06-2000 & - & 4 & 81.5 & 8.94 \\
\hline & Mixed fish & 06-03-1999 & 4 & 15 & 79.5 & 12.3 \\
\hline & Mixed fish & 06-08-2000 & - & 11 & 81.9 & 9.67 \\
\hline & Red Shiner & 06-02-1999 & - & 49 & 72.8 & 28.8 \\
\hline & Red Shiner & 06-22-1999 & 9 & 21 & 71.9 & 11.9 \\
\hline & Red Shiner & 07-21-1999 & 50 & 152 & 77.9 & 14.9 \\
\hline \multirow[t]{2}{*}{ SLJ1 } & Common Carp & 06-30-1998 & 25 & 159 & 71.2 & 13.1 \\
\hline & Fathead Minnow & 04-21-1998 & - & 21 & 77.2 & 7.08 \\
\hline \multirow[t]{2}{*}{ SSD } & Common Carp & 07-26-1999 & - & 21 & 80.0 & 16.5 \\
\hline & Larval fish & 07-26-1999 & - & 2 & 75.5 & 12.2 \\
\hline SFP & Larval fish & 06-09-2000 & - & 5 & 87.4 & 13.3 \\
\hline \multirow[t]{3}{*}{ SLM } & Larval fish & 06-01-2000 & - & 2 & 93.0 & 11.1 \\
\hline & Larval fish & 06-01-2000 & - & 2 & 86.8 & 12.8 \\
\hline & Larval fish & $06-06-2000$ & - & 3 & 88.3 & 10.1 \\
\hline
\end{tabular}


Table 32. Concentration of selenium in nonendangered fish samples except common carp collected from Stewart Lake Waterfowl Management Area near Jensen, Utah, 1994-2000

[Data from U.S. Fish and Wildlife Service; $\mu \mathrm{g} / \mathrm{g}$, micrograms per gram, dry weight; 一, not recorded; see table 30 for common carp]

\begin{tabular}{|c|c|c|c|c|c|c|}
\hline $\begin{array}{l}\text { Abbreviated } \\
\text { site name }\end{array}$ & Species & Date & Number of fish & $\begin{array}{l}\text { Weight } \\
\text { (grams) }\end{array}$ & $\begin{array}{l}\text { Percent } \\
\text { moisture }\end{array}$ & $\begin{array}{l}\text { Selenium } \\
(\mu \mathrm{g} / \mathrm{g})\end{array}$ \\
\hline \multirow[t]{20}{*}{ SLN } & Black Bullhead & 06-16-1998 & 7 & 661 & 80.3 & 9.3 \\
\hline & Black Bullhead & 07-08-1999 & 5 & 354 & 80.4 & 13.0 \\
\hline & Channel Catfish & 10-26-1994 & - & 650 & 74.5 & 12.0 \\
\hline & Channel Catfish & 05-17-1995 & - & 3,671 & 74.3 & 15.6 \\
\hline & Channel Catfish & 07-13-1995 & - & 2,233 & 76.5 & 14.2 \\
\hline & Channel Catfish & 07-13-1995 & - & 615 & 73.6 & 8.03 \\
\hline & Channel Catfish & 04-23-1996 & 1 & 244 & 78.2 & 18.2 \\
\hline & Channel Catfish & 04-23-1996 & 1 & 778 & 72.4 & 15.0 \\
\hline & Channel Catfish & 05-28-1997 & 2 & 877 & 76.1 & 10.2 \\
\hline & Channel Catfish & 06-10-1997 & 2 & 1,811 & 73.2 & 10.2 \\
\hline & Channel Catfish & 06-22-1999 & 3 & 1,289 & 76.5 & 7.14 \\
\hline & Channel Catfish & 07-08-1999 & 3 & 1,284 & 77.5 & 7.00 \\
\hline & Channel Catfish & 07-20-1999 & 3 & 2,748 & 75.5 & 7.91 \\
\hline & Channel Catfish & $05-31-2000$ & 5 & 1,444 & 77.0 & 8.45 \\
\hline & Northern Pike & 04-29-1998 & 4 & 5,391 & 75.8 & 5.46 \\
\hline & White Sucker & 10-26-1994 & - & 2,203 & 75.2 & 18.1 \\
\hline & White Sucker & 05-17-1995 & - & 2,248 & 73.3 & 16.4 \\
\hline & White Sucker & 07-13-1995 & - & 2,444 & 74.6 & 13.2 \\
\hline & White Sucker & $11-28-1995$ & 4 & 1,987 & 72.7 & 22.1 \\
\hline & White Sucker & $10-24-1996$ & 1 & 184 & 79.8 & 24.8 \\
\hline \multirow[t]{13}{*}{ SLO } & Black Bullhead & 04-15-1997 & 4 & 131 & 82.6 & 18.0 \\
\hline & Black Bullhead & 07-15-1998 & 5 & 681 & 81.2 & 5.37 \\
\hline & Black Bullhead & 07-20-1999 & 25 & 51 & 83.6 & 21.1 \\
\hline & Channel Catfish & 05-17-1995 & - & 3,671 & 73.8 & 11.1 \\
\hline & Channel Catfish & 07-12-1995 & - & 2,501 & 74.4 & 9.17 \\
\hline & Channel Catfish & 07-20-1999 & 2 & 740 & 80.2 & 7.59 \\
\hline & Channel Catfish & 06-01-2000 & 3 & 580 & 79.1 & 20.3 \\
\hline & Channel Catfish & 06-02-2000 & 3 & 629 & 76.9 & 11.0 \\
\hline & Northern Pike & 04-21-1998 & 3 & 4,426 & 75.2 & 6.92 \\
\hline & White Sucker & 05-17-1995 & 一 & 2,455 & 72.8 & 20.0 \\
\hline & White Sucker & 04-23-1996 & 5 & 2,095 & 72.3 & 28.6 \\
\hline & White Sucker & 10-24-1996 & 1 & 337 & 74.8 & 17.0 \\
\hline & White Sucker & 06-10-1997 & 3 & 1,802 & 73.7 & 3.72 \\
\hline SLI & Channel Catfish & $06-28-2000$ & 4 & 2,172 & 78.0 & 9.66 \\
\hline SLJ1 & Black Bullhead & 08-28-1997 & 4 & 135 & 83.3 & 9.60 \\
\hline SSD & Black Bullhead & 07-26-1999 & - & 17 & 84.1 & 8.70 \\
\hline \multirow[t]{2}{*}{ SLM } & Channel Catfish & 06-18-1997 & muscle plug & - & - & 19.4 \\
\hline & Black Bullhead & 07-20-1999 & 50 & 29 & 84.6 & 22.0 \\
\hline
\end{tabular}


Table 33. Concentration of selenium in bird eggs collected from Stewart Lake Waterfowl Management Area near Jensen, Utah, 1995-2000

[Data from U.S. Fish and Wildlife Service; $\mu \mathrm{g} / \mathrm{g}$, micrograms per gram, dry weight]

\begin{tabular}{|c|c|c|c|c|c|}
\hline $\begin{array}{c}\text { Abbreviated site } \\
\text { name }\end{array}$ & Species & Date & $\begin{array}{l}\text { Total weight } \\
\text { (grams) }\end{array}$ & $\begin{array}{c}\text { Percent } \\
\text { moisture }\end{array}$ & $\begin{array}{c}\text { Selenium } \\
(\mu \mathrm{g} / \mathrm{g})\end{array}$ \\
\hline \multirow[t]{2}{*}{$\mathrm{BB}$} & American Coot & $06-24-1997$ & 19 & 73.1 & 2.21 \\
\hline & American Coot & 06-24-1997 & 27 & 76.4 & 1.23 \\
\hline \multirow[t]{15}{*}{ SLM } & American Coot & 06-07-1995 & 30 & 76.5 & 19.0 \\
\hline & American Coot & 06-07-1995 & 22 & 72.5 & 13.6 \\
\hline & American Coot & 06-22-1999 & 26 & 74.5 & 13.5 \\
\hline & Black-necked Stilt & $06-27-2000$ & 16 & 73.0 & 15.5 \\
\hline & Black-necked Stilt & $06-27-2000$ & 18 & 73.6 & 14.6 \\
\hline & Black-necked Stilt & $06-27-2000$ & 10 & 73.8 & 8.01 \\
\hline & Black-necked Stilt & $06-27-2000$ & 17 & 71.8 & 15.7 \\
\hline & Canada Goose & 05-03-1995 & 135 & 70.1 & 4.13 \\
\hline & Canada Goose & 05-03-1995 & 124 & 68.2 & 3.90 \\
\hline & Canada Goose & 05-03-1995 & 123 & 68.0 & 1.63 \\
\hline & Redhead & 05-13-1995 & 48 & 68.3 & 3.63 \\
\hline & Western Grebe & 06-07-1995 & 35 & 75.7 & 24.4 \\
\hline & Western Grebe & 06-07-1995 & 36 & 76.7 & 24.1 \\
\hline & Western Grebe & 06-07-1995 & 35 & 76.0 & 21.0 \\
\hline & Western Grebe & 06-07-1995 & 36 & 74.7 & 19.9 \\
\hline \multirow[t]{6}{*}{ SLN } & American Coot & 07-07-1999 & 20 & 75.2 & 23.6 \\
\hline & American Coot & 07-21-1999 & 19 & 78.0 & 22.3 \\
\hline & American Coot & $06-28-2000$ & 26 & 77.4 & 25.4 \\
\hline & Forster's Tern & $06-28-2000$ & 16 & 79.2 & 14.4 \\
\hline & Redhead & 06-11-1997 & 47 & 67.1 & 14.6 \\
\hline & Western Grebe & $06-27-2000$ & 45 & 79.3 & 15.7 \\
\hline \multirow[t]{6}{*}{ SLO } & American Avocet & 06-16-1998 & 23 & 74.0 & 24.5 \\
\hline & American Avocet & 06-16-1998 & 26 & 74.2 & 16.6 \\
\hline & American Coot & $06-27-2000$ & 18 & 77.2 & 15.8 \\
\hline & Eared Grebe & $06-27-2000$ & 19 & 77.0 & 26.6 \\
\hline & Redhead & 06-10-1997 & 40 & 71.4 & 3.60 \\
\hline & Redhead & 06-11-1997 & 41 & 69.1 & 6.90 \\
\hline \multirow[t]{3}{*}{ SSD } & Black-necked Stilt & 06-22-1999 & 18 & 73.6 & 14.8 \\
\hline & Killdeer & 06-23-1999 & 13 & 72.1 & 12.9 \\
\hline & Killdeer & 07-08-1999 & 11 & 72.8 & 13.7 \\
\hline
\end{tabular}

Table 34. Concentration of selenium in bird livers collected from Stewart Lake Waterfowl Management Area near Jensen, Utah, 1995

[Data from U.S. Fish and Wildlife Service; $\mu \mathrm{g} / \mathrm{g}$, micrograms per gram, dry weight]

\begin{tabular}{cccccc}
\hline $\begin{array}{c}\text { Abbreviated site } \\
\text { name }\end{array}$ & Species & Date & $\begin{array}{c}\text { Total weight } \\
(\text { grams })\end{array}$ & $\begin{array}{c}\text { Percent } \\
\text { moisture }\end{array}$ & $\begin{array}{c}\text { Selenium } \\
(\mu \mathbf{g} / \mathbf{g})\end{array}$ \\
\hline SLM & American Coot & $11-28-1995$ & 17 & 69.2 & 49.1 \\
& American Coot & $11-28-1995$ & 15 & 68.5 & 38.7 \\
& American Coot & $11-28-1995$ & 12 & 69.7 & 32.3 \\
& American Coot & $11-28-1995$ & 44 & 72.0 & 18.0 \\
& American Coot & $11-28-1995$ & 44 & 70.0 & 15.6 \\
& American Coot & $11-28-1995$ & 33 & 66.3 & 15.1 \\
& Canada Goose & $11-28-1995$ & 31 & 78.7 & 15.3 \\
\hline
\end{tabular}


Table 35. Concentration of trace elements in biological samples collected from selected sites in the middle Green River basin, eastern Utah (except Stewart Lake Waterfowl Management Area), 1991-93

[Data from U.S. Fish and Wildlife Service; g, grams; $\mu \mathrm{g} / \mathrm{g}$, micrograms per gram, dry weight; <, less than; WB, Whole body; -, not determined]

\begin{tabular}{|c|c|c|c|c|c|c|c|c|c|c|c|}
\hline $\begin{array}{c}\text { Short site } \\
\text { name }\end{array}$ & Species & Date & Sample type & $\begin{array}{l}\text { Total } \\
\text { weight } \\
\text { (g) }\end{array}$ & $\begin{array}{l}\text { Percent } \\
\text { moisture }\end{array}$ & $\begin{array}{c}\text { Alumi- } \\
\text { num } \\
(\mu \mathrm{g} / \mathrm{g})\end{array}$ & $\begin{array}{c}\text { Arsenic } \\
(\mu \mathrm{g} / \mathrm{g})\end{array}$ & $\begin{array}{l}\text { Boron } \\
(\mu \mathrm{g} / \mathrm{g})\end{array}$ & $\begin{array}{c}\text { Barium } \\
(\mu g / g)\end{array}$ & $\begin{array}{c}\text { Beryllium } \\
(\mu \mathrm{g} / \mathrm{g})\end{array}$ & $\underset{(\mu \mathrm{g} / \mathrm{g})}{\text { Cadmium }}$ \\
\hline $30 \mathrm{~A}$ & Filamentous Green Algae & 08-10-1993 & Vegetation & 54 & 93.2 & 3,220 & 10.0 & 14.9 & 699 & $<0.240$ & 0.479 \\
\hline $30 \mathrm{~A}$ & Stonewart & 08-10-1993 & Vegetation & 82 & 89.2 & 539 & 2.11 & 7.28 & 60.8 & $<.242$ & $<.242$ \\
\hline $30 \mathrm{~A}$ & Pondweed & 08-10-1993 & Vegetation & 104 & 90.8 & 843 & 3.28 & 21.8 & 93.3 & $<.246$ & .2703 \\
\hline $30 \mathrm{~A}$ & Watercress & 08-10-1993 & Vegetation & 89 & 95.5 & 389 & 1.80 & 19.9 & 86.0 & $<.235$ & $<.235$ \\
\hline $30 \mathrm{~A}$ & Filamentous Green Algae & 08-10-1993 & Vegetation & 40 & 87.8 & 321 & 6.55 & 192 & 11.5 & $<.244$ & $<.244$ \\
\hline $30 \mathrm{~A}$ & Filamentous Green Algae & 08-10-1993 & Vegetation & 56 & 88.6 & 400 & 6.25 & 104 & 16.3 & $<.238$ & $<.238$ \\
\hline $30 \mathrm{~A}$ & Watercress & 08-10-1993 & Vegetation & 46 & 96.5 & 562 & 2.64 & 32.8 & 14.8 & $<.243$ & .387 \\
\hline $\mathrm{AC} 40$ & Cliff Swallow & 07-09-1991 & WB & 25 & 76.3 & 549 & .33 & 6 & 36.7 & .02 & .19 \\
\hline $\mathrm{AC} 40$ & Cliff Swallow & 07-09-1991 & WB & 67 & 79.5 & 260 & $<.1$ & 5 & 9.7 & $<.01$ & .12 \\
\hline $\mathrm{AC} 40$ & Waterboatmen & 08-21-1991 & Invertebrate & 3 & 89.8 & & & & & & \\
\hline AC40 & Crayfish & 08-15-1991 & Invertebrate & 145 & 77.3 & 521 & 2 & 5 & 35.6 & .02 & .38 \\
\hline $\mathrm{AC} 40$ & Red Shiner & 08-15-1991 & Fish, WB & 56 & 76.0 & 270 & .4 & $<2$ & 3.7 & $<.01$ & .06 \\
\hline $\mathrm{AC} 40$ & Dace, probably speckled & 08-15-1991 & Fish, WB & 55 & 69.5 & 76 & .3 & $<2$ & 2.2 & $<.01$ & .086 \\
\hline AC40 & White Sucker & 08-15-1991 & Fish, WB & 534 & 75.4 & 98 & .3 & $<2$ & 1.9 & $<.01$ & $<.03$ \\
\hline ACBJ & Crayfish & 08-16-1991 & Invertebrate & 49 & 72.5 & 120 & 2 & 4 & 15.7 & $<.03$ & .36 \\
\hline ACBJ & Fathead Minnow & 08-16-1991 & Fish, WB & 27 & 76.3 & 2,650 & 1 & 4 & 27.9 & .093 & .11 \\
\hline ACBJ & Red Shiner & 08-16-1991 & Fish, WB & 52 & 75.0 & 42 & .6 & $<2$ & 1.4 & $<.01$ & .06 \\
\hline ACBR & Crayfish & 08-15-1991 & Invertebrate & 105 & 76.7 & 429 & 2.8 & 4 & 33.8 & $<.03$ & .4 \\
\hline ACBR & Red Shiner & 08-15-1991 & Fish, WB & 75 & 76.0 & 907 & .6 & $<2$ & 8.1 & .03 & .19 \\
\hline ACBR & Dace, probably speckled & 08-15-1991 & Fish, WB & 19 & 71.0 & 671 & .3 & $<2$ & 6 & .02 & .42 \\
\hline ACBR & Cattail & 05-09-1991 & Vegetation & 185 & 85.9 & 466 & .87 & 13 & 52.5 & .03 & .06 \\
\hline ACBR & Red Shiner & 08-20-1991 & Fish, WB & 935 & 92.9 & 734 & 2.3 & 20 & 21.5 & .03 & .38 \\
\hline ACBR & White Sucker & 08-15-1991 & Fish, WB & 519 & 75.0 & 240 & .3 & $<2$ & 2.9 & $<.01$ & .04 \\
\hline $\mathrm{ACN}$ & Cliff Swallow & 07-09-1991 & WB & 23 & 56.2 & 260 & $<.1$ & $<2$ & 4.3 & $<.01$ & .14 \\
\hline $\mathrm{ACN}$ & Cliff Swallow & 07-09-1991 & WB & 56 & 64.4 & 280 & $<.1$ & 2 & 6.5 & $<.01$ & .11 \\
\hline $\mathrm{ACN}$ & Canada Goose & 05-09-1991 & Not Recorded & 26 & 72.1 & 120 & .1 & 2 & 7 & $<.01$ & .04 \\
\hline ACS & Cliff Swallow & 07-09-1991 & WB & 88 & 65.2 & 190 & .1 & $<2$ & 10.8 & $<.01$ & .093 \\
\hline $\mathrm{ACV}$ & Corixids & 08-21-1991 & Invertebrate & 21 & 85.3 & 65 & .68 & 4 & 1.4 & $<.01$ & $<.03$ \\
\hline $\mathrm{ACV}$ & Pondweed & 05-09-1991 & Vegetation & 50 & 91.4 & 1,870 & 5.6 & 21 & 46.5 & .074 & .2 \\
\hline $\mathrm{ACV}$ & Cattail & 05-09-1991 & Vegetation & 197 & 87.2 & 709 & 2.9 & 12 & 22.8 & .037 & .099 \\
\hline $\mathrm{ACV}$ & Crayfish & 08-15-1991 & Invertebrate & 134 & 78.2 & 240 & 2.3 & $<2$ & 52.4 & $<.01$ & 1.7 \\
\hline $\mathrm{ACV}$ & Fathead Minnow & 08-15-1991 & Fish, WB & 17 & 76.4 & 490 & .83 & $<2$ & 7.5 & .01 & .44 \\
\hline $\mathrm{ACV}$ & Dace, probably speckled & 08-15-1991 & Fish, WB & 10 & 76.5 & 120 & .3 & $<2$ & 4.6 & $<.01$ & .37 \\
\hline ACV & Pondweed & 08-21-1991 & Vegetation & 100 & 90.3 & 1,620 & 2.6 & 28 & 36 & .065 & .13 \\
\hline $\mathrm{ACV}$ & Cattail & 08-21-1991 & Vegetation & 388 & 90.8 & 660 & 1.6 & 14 & 10.9 & .02 & .07 \\
\hline $\mathrm{AVCP}$ & Filamentous Green Algae & 08-10-1993 & Vegetation & 35 & 94.0 & 313 & 1.4 & 136 & 53.5 & $<.249$ & $<.249$ \\
\hline $\mathrm{AVCP}$ & Filamentous Green Algae & 08-10-1993 & Vegetation & 61 & 89.6 & 1,190 & 6.46 & 52.3 & 1,190 & $<.234$ & $<.234$ \\
\hline $\mathrm{AVCP}$ & Black Bullhead & $08-10-1993$ & Fish, WB & 643 & 78.2 & 100 & $<.485$ & 1.14 & 23.5 & $<.099$ & $<.099$ \\
\hline $\mathrm{AVCP}$ & Stonewart & 08-10-1993 & Vegetation & 38 & 88.5 & 1,010 & 4.29 & 12.0 & 381.4 & .256 & .278 \\
\hline $\mathrm{AVCP}$ & Fathead Minnow & 08-10-1993 & Fish, WB & 13 & 81.2 & 607 & 1.42 & 3.16 & 20.5 & $<.206$ & $<.206$ \\
\hline $\mathrm{AVCP}$ & Green Sunfish & 08-10-1993 & Fish, WB & 49 & 80.1 & 48.5 & $<.499$ & 1.62 & 3.18 & $<.097$ & $<.097$ \\
\hline $\mathrm{AVCP}$ & White Sucker & 08-10-1993 & Fish, WB & 706 & 75.7 & 134 & .589 & .744 & 10.3 & $<.100$ & $<.098$ \\
\hline $\mathrm{AVCP}$ & White Sucker & 08-10-1993 & Fish, WB & 642 & 75.2 & 80.2 & .553 & $<.477$ & 12.2 & $<.095$ & $<.095$ \\
\hline $\mathrm{AVCP}$ & Cattail & 08-10-1993 & Vegetation & 378 & 89.9 & 447 & 1.81 & 12.7 & 23.3 & $<.249$ & $<.249$ \\
\hline ACGR & Pondweed & 08-20-1991 & Vegetation & 50 & 90.0 & 3,380 & 2.6 & 280 & 38.1 & .12 & .21 \\
\hline ACGR & Cattail & 05-09-1991 & Vegetation & 55 & 91.9 & 1,130 & 3.7 & 19 & 18.7 & .053 & .18 \\
\hline ACGR & Cattail & 08-20-1991 & Vegetation & 39 & 92.5 & 1,010 & .97 & 27 & 14.3 & .036 & .21 \\
\hline ACGR & Crayfish & 08-16-1991 & Invertebrate & 7 & 99.2 & 690 & $<2$ & $<30$ & 18 & $<.4$ & $<1$ \\
\hline ACGR & Fathead Minnow & 08-16-1991 & Fish, WB & 5 & 79.4 & 310 & .36 & 3 & 15.3 & $<.03$ & .2 \\
\hline ACGR & Green Sunfish & 08-16-1991 & Fish, WB & 145 & 75.8 & 43 & $<.2$ & $<2$ & 2.4 & $<.01$ & .03 \\
\hline ACGR & Red Shiner & 08-16-1991 & Fish, WB & 7 & 77.7 & 810 & .5 & $<2$ & 14 & .03 & .16 \\
\hline ACGR & Algae & 08-11-1993 & Vegetation & 40 & 45.2 & 6,020 & 5.94 & 7.45 & 133 & .383 & .406 \\
\hline $\mathrm{BC}$ & Waterboatmen & 07-13-1993 & Invertebrate & 2 & 74.6 & 618 & 1.91 & 1.26 & 16.5 & $<.235$ & 1.00 \\
\hline $\mathrm{BCD}$ & Crayfish & 08-27-1991 & Invertebrate & 164 & 72.3 & 846 & 2.1 & 3 & 133 & .034 & .45 \\
\hline $\mathrm{BCD}$ & Fathead Minnow & 08-27-1991 & Fish, WB & 49 & 78.2 & 2,320 & 1.2 & 2 & 32.4 & .083 & .53 \\
\hline
\end{tabular}


Table 35. Concentration of trace elements in biological samples collected from selected sites in the middle Green River basin, eastern Utah (except Stewart Lake Waterfowl Management Area), 1991-93_Continued

\begin{tabular}{|c|c|c|c|c|c|c|c|c|c|c|c|c|c|}
\hline $\begin{array}{l}\text { Short site } \\
\text { name }\end{array}$ & $\underset{(\mu \mathrm{g} / \mathrm{g})}{\text { Chromium }}$ & $\begin{array}{c}\text { Copper } \\
(\mu \mathrm{g} / \mathrm{g})\end{array}$ & $\begin{array}{l}\text { Iron } \\
(\mu \mathrm{g} / \mathrm{g})\end{array}$ & $\begin{array}{c}\text { Mercury } \\
(\mu \mathrm{g} / \mathrm{g})\end{array}$ & $\underset{(\mu \mathrm{g} / \mathrm{g})}{\text { Magnesium }}$ & $\begin{array}{c}\text { Manga- } \\
\text { nese } \\
(\mu \mathrm{g} / \mathrm{g})\end{array}$ & $\begin{array}{c}\text { Molyb- } \\
\text { denum } \\
(\mu \mathrm{g} / \mathrm{g})\end{array}$ & $\begin{array}{l}\text { Nickel } \\
(\mu \mathrm{g} / \mathrm{g})\end{array}$ & $\begin{array}{l}\text { Lead } \\
(\mu \mathrm{g} / \mathrm{g})\end{array}$ & $\begin{array}{c}\text { Selenium } \\
(\mu \mathrm{g} / \mathrm{g})\end{array}$ & $\begin{array}{c}\text { Strontium } \\
(\mu \mathrm{g} / \mathrm{g})\end{array}$ & $\underset{(\mu \mathrm{g} / \mathrm{g})}{\text { Vanadium }}$ & $\begin{array}{l}\text { Zinc } \\
(\mu \mathrm{g} / \mathrm{g})\end{array}$ \\
\hline $30 \mathrm{~A}$ & 11.91 & 12.2 & 5,880 & $<0.245$ & 6,400 & 753 & $<1.20$ & 7.46 & 9.74 & 5.86 & 381 & 13.7 & 68.6 \\
\hline $30 \mathrm{~A}$ & 3.46 & 3.84 & 962 & $<.246$ & 6,500 & 1,410 & $<1.21$ & 3.18 & 4.79 & $<1.23$ & 1,570 & 2.82 & 20.4 \\
\hline $30 \mathrm{~A}$ & 6.46 & 6.15 & 2,380 & $<.25$ & 3,030 & 1,140 & 2.09 & 4.66 & 2.86 & 3.28 & 285 & 7.17 & 34.7 \\
\hline $30 \mathrm{~A}$ & 4.64 & 7.95 & 1,040 & $<.235$ & 3,940 & 362 & 2.72 & 2.46 & 2.65 & 2.58 & 240 & 2.71 & 56.2 \\
\hline $30 \mathrm{~A}$ & 4.09 & 2.89 & 1,100 & $<.246$ & 4,210 & 211 & $<1.22$ & 1.80 & 2.41 & 2.00 & 753 & $<1.22$ & 20.9 \\
\hline $30 \mathrm{~A}$ & 5.72 & 3.21 & 2,740 & $<.242$ & 3,760 & 372 & $<1.19$ & 4.26 & 1.92 & 4.37 & 671 & 1.65 & 27.5 \\
\hline $30 \mathrm{~A}$ & 5.49 & 5.30 & 3,320 & $<.248$ & 8,023 & 170 & 1.64 & 5.77 & 2.89 & 5.17 & 349 & 2.88 & 69.8 \\
\hline $\mathrm{AC} 40$ & 2.9 & 12 & 475 & .02 & 1,770 & 20.2 & $<1$ & 1.6 & .73 & 4.4 & 45.9 & 1.5 & 109 \\
\hline $\mathrm{AC} 40$ & 5.2 & 14 & 368 & .02 & 1,300 & 11 & $<1$ & 2.9 & .46 & 3.4 & 26.8 & 1 & 112 \\
\hline AC40 & - & - & - & - & - & - & - & - & - & 22.0 & - & - & - \\
\hline AC40 & 2.1 & 40.5 & 317 & .018 & 2,380 & 131 & $<1$ & 2.1 & .5 & 13.0 & 1,120 & 2.6 & 52 \\
\hline AC40 & 1.9 & 4.5 & 237 & .051 & 1,530 & 33.5 & $<1$ & 1.1 & $<.4$ & 41.0 & 212 & 1.1 & 196 \\
\hline $\mathrm{AC} 40$ & .57 & 2.4 & 111 & .037 & 1,160 & 26.7 & $<.9$ & .3 & $<.4$ & 38.0 & 172 & .4 & 141 \\
\hline $\mathrm{AC} 40$ & 1.2 & 3.3 & 144 & .032 & 1,330 & 28 & $<1$ & .62 & $<.5$ & 64.0 & 143 & .6 & 72.6 \\
\hline ACBJ & 1.7 & 48.8 & 231 & .04 & 3,020 & 122 & $<1$ & 2.2 & $<1$ & 12.0 & 1,130 & .4 & 54.2 \\
\hline ACBJ & 6.7 & 4.6 & 1,430 & .025 & 2,590 & 67.8 & $<.9$ & 3.3 & 1 & 26.0 & 163 & 7.7 & 121 \\
\hline ACBJ & 1.2 & 2.6 & 145 & .053 & 1,310 & 19.7 & $<.9$ & .6 & $<.4$ & 34.0 & 178 & $<.3$ & 160 \\
\hline ACBR & 1.1 & 56.6 & 255 & .027 & 2,870 & 171 & $<1$ & 1.6 & $<1$ & 16.0 & 926 & 1.6 & 57.7 \\
\hline ACBR & 2.4 & 2.9 & 558 & .037 & 1,790 & 69.6 & $<.9$ & 1.4 & .5 & 41.0 & 221 & 3.3 & 170 \\
\hline ACBR & .88 & 1.9 & 413 & .076 & 1,410 & 61.1 & $<1$ & .4 & $<.5$ & 35.0 & 152 & 2.4 & 106 \\
\hline ACBR & 12.2 & 2.2 & 1,170 & .01 & 2,690 & 139 & 1 & 6.4 & .7 & 13.0 & 84.5 & 3.5 & 15 \\
\hline ACBR & 3.6 & 2.8 & 2,120 & .3 & 3,910 & 130 & $<1$ & 2.2 & .7 & 5.2 & 139 & 3.2 & 18 \\
\hline ACBR & 1.2 & 3.7 & 227 & .046 & 1,280 & 25.1 & $<1$ & .65 & 2.4 & 64.0 & 119 & .87 & 63.5 \\
\hline $\mathrm{ACN}$ & 3.1 & 6 & 240 & .082 & 796 & 8.8 & $<1$ & 1.9 & .2 & 13 & 38 & .9 & 72.7 \\
\hline $\mathrm{ACN}$ & 6.8 & 5.8 & 342 & .062 & 1,030 & 8.4 & $<1$ & 3.1 & .2 & 14 & 49.3 & .92 & 82.3 \\
\hline $\mathrm{ACN}$ & 11 & 8.6 & 409 & .13 & 850 & 10 & $<1$ & 5.5 & $<.1$ & 24 & 59.3 & .5 & 87.7 \\
\hline ACS & 8.9 & 6 & 334 & .035 & 857 & 7.6 & $<1$ & 4.1 & 1.1 & 11 & 55 & .8 & 73.6 \\
\hline $\mathrm{ACV}$ & $<.1$ & 8.4 & 243 & .087 & 1,270 & 43.4 & $<1$ & .4 & $<.4$ & 4.2 & 27.4 & $<.3$ & 113 \\
\hline $\mathrm{ACV}$ & 3.4 & 6.2 & 6,580 & .027 & 4,100 & 629 & 2 & 3.5 & 1.5 & 4.1 & 357 & 7.5 & 49 \\
\hline $\mathrm{ACV}$ & 2.1 & 2.7 & 3,330 & .009 & 3,170 & 267 & $<1$ & 1.9 & .5 & 1.1 & 120 & 3.3 & 25 \\
\hline $\mathrm{ACV}$ & .57 & 40.6 & 329 & .069 & 2,660 & 84.1 & $<1$ & .77 & $<.4$ & 2.2 & 965 & .6 & 62.9 \\
\hline $\mathrm{ACV}$ & .75 & 3.5 & 404 & .16 & 1,600 & 38.3 & $<1$ & .3 & 1 & 7.9 & 163 & 1.3 & 141 \\
\hline ACV & .3 & 3.3 & 124 & .42 & 100 & 18 & $<1$ & $<.1$ & $<.4$ & 11.0 & 295 & $<.3$ & 189 \\
\hline $\mathrm{ACV}$ & 3.9 & 5.8 & 3,720 & .027 & 4,020 & 239 & 3 & 3.1 & 1 & 3.0 & 332 & 6.7 & 22 \\
\hline $\mathrm{ACV}$ & 1.7 & 3.2 & 1,540 & .01 & 3,370 & 169 & $<1$ & 1.5 & 2.6 & 1.2 & 142 & 3.3 & 15 \\
\hline $\mathrm{AVCP}$ & 1.85 & 2.98 & 467 & $<.240$ & 12,500 & 344 & $<1.24$ & 1.93 & 2.42 & 1.64 & 442 & 1.24 & 10.1 \\
\hline $\mathrm{AVCP}$ & 5.06 & 7.27 & 2,610 & $<.242$ & 4,920 & 764 & $<1.17$ & 5.29 & 7.07 & 1.26 & 650 & 4.43 & 35.7 \\
\hline $\mathrm{AVCP}$ & 4.09 & 3.53 & 151 & .118 & 1,560 & 27.4 & $<.494$ & .59 & .755 & 2.90 & 124 & $<.494$ & 88.8 \\
\hline $\mathrm{AVCP}$ & 3.50 & 5.63 & 2,650 & $<.245$ & 5,520 & 513 & $<1.19$ & 5.24 & 8.53 & 2.38 & 1,245 & 4.76 & 24.8 \\
\hline $\mathrm{AVCP}$ & 5.77 & 8.00 & 598 & $<.206$ & 1,640 & 31.1 & $<1.03$ & $<1.03$ & $<1.03$ & 2.94 & 142 & 2.09 & 169 \\
\hline AVCP & 9.46 & 2.72 & 168 & .141 & 1,470 & 12.5 & $<.486$ & .784 & .643 & 4.88 & 123 & $<.486$ & 124 \\
\hline $\mathrm{AVCP}$ & 18.0 & 4.73 & 287 & $<.098$ & 1,460 & 15.1 & .568 & .609 & .553 & 2.94 & 126 & .513 & 86.3 \\
\hline $\mathrm{AVCP}$ & 4.12 & 4.32 & 143 & $<.097$ & 1,569 & 8.23 & $<.477$ & $<.477$ & $<.477$ & 3.30 & 147 & $<.477$ & 94.2 \\
\hline $\mathrm{AVCP}$ & 6.54 & 3.60 & 2,470 & $<.242$ & 3,160 & 134 & $<1.24$ & 2.29 & $<1.24$ & 1.79 & 139 & 3.03 & 21.6 \\
\hline ACGR & 4.6 & 5.8 & 2,910 & .016 & 8,010 & 1,910 & 4.9 & 5.1 & 2 & 13.0 & 250 & 13 & 40.5 \\
\hline ACGR & 8 & 6.9 & 4,290 & .01 & 4,220 & 185 & $<1$ & 4.4 & .9 & 4.5 & 134 & 5.3 & 61.7 \\
\hline ACGR & 2.9 & 7.8 & 1,330 & .02 & 6,000 & 384 & 2 & 2.3 & .7 & 3.5 & 171 & 4.5 & 71.8 \\
\hline ACGR & $<4$ & 120 & 680 & .1 & 2,520 & 150 & $<20$ & $<6$ & $<20$ & 19.0 & 383 & $<5$ & 120 \\
\hline ACGR & .7 & 3.1 & 541 & .098 & 1,640 & 68.9 & $<1$ & $<.3$ & $<1$ & 24.0 & 183 & 1.3 & 167 \\
\hline ACGR & 1.1 & 1.2 & 76 & .19 & 1,530 & 13 & $<1$ & .55 & $<.4$ & 20.0 & 145 & $<.3$ & 83.8 \\
\hline ACGR & 2.4 & 3.5 & 504 & .073 & 18,80 & 27.7 & $<.9$ & 1.4 & .5 & 19.0 & 181 & 2.5 & 195 \\
\hline ACGR & 101 & 12.5 & 10,400 & $<.098$ & 11,300 & 538 & $<4.89$ & 14.0 & 8.87 & 6.63 & 224 & 17.4 & 86.3 \\
\hline $\mathrm{BC}$ & 2.04 & 19.5 & 1,540 & $<.239$ & 1,550 & 90.1 & $<1.18$ & 1.82 & $<1.17$ & 4.95 & 11.6 & 2.31 & 118 \\
\hline $\mathrm{BCD}$ & 1.7 & 80.1 & 488 & .077 & 2,090 & 153 & $<1$ & 1.6 & $<.5$ & 4.6 & 565 & 2.1 & 59.8 \\
\hline $\mathrm{BCD}$ & 2.9 & 3.8 & 1,320 & .13 & 2,290 & 45 & $<1$ & 1.6 & 1 & 18.0 & 90.4 & 6.2 & 123 \\
\hline
\end{tabular}


Table 35. Concentration of trace elements in biological samples collected from selected sites in the middle Green River basin, eastern Utah (except Stewart Lake Waterfowl Management Area), 1991-93_Continued

\begin{tabular}{|c|c|c|c|c|c|c|c|c|c|c|c|}
\hline $\begin{array}{c}\text { Short site } \\
\text { name }\end{array}$ & Species & Date & Sample type & $\begin{array}{l}\text { Total } \\
\text { weight } \\
\text { (g) }\end{array}$ & $\begin{array}{l}\text { Percent } \\
\text { moisture }\end{array}$ & $\begin{array}{l}\text { Alumi- } \\
\text { num } \\
(\mu \mathrm{g} / \mathrm{g})\end{array}$ & $\begin{array}{c}\text { Arsenic } \\
(\mu \mathrm{g} / \mathrm{g})\end{array}$ & $\begin{array}{l}\text { Boron } \\
(\mu \mathrm{g} / \mathrm{g})\end{array}$ & $\begin{array}{c}\text { Barium } \\
(\mu \mathrm{g} / \mathrm{g})\end{array}$ & $\begin{array}{l}\text { Beryllium } \\
(\mu \mathrm{g} / \mathrm{g})\end{array}$ & $\underset{(\mu \mathrm{g} / \mathrm{g})}{\text { Cadmium }}$ \\
\hline $\mathrm{BCD}$ & Green Sunfish & $08-27-1991$ & Fish, WB & 169 & 75.3 & 93 & .3 & $<2$ & 6 & $<.01$ & .05 \\
\hline $\mathrm{BCD}$ & Mountain Sucker & 08-27-1991 & Fish, WB & 148 & 76.2 & 2,450 & .69 & $<2$ & 23 & .087 & .11 \\
\hline BC149 & Pondweed & 08-21-1991 & Vegetation & 60 & 87.7 & 3,110 & 9.1 & 257 & 103 & .12 & .53 \\
\hline BC149 & Speckled Dace & 08-27-1991 & Fish, WB & 40 & 77.0 & 336 & .3 & $<2$ & 10.3 & .01 & .38 \\
\hline BC149 & Cattail & 08-21-1991 & Vegetation & 538 & 85.2 & 1,110 & .5 & 11 & 17.4 & .037 & .05 \\
\hline BC149 & White Sucker & 08-27-1991 & Fish, WB & 624 & 78.1 & 375 & .5 & $<2$ & 8.3 & .01 & .097 \\
\hline BIP & Green Sunfish & 07-24-1991 & Fish, WB & 314 & 75.3 & $<3$ & .1 & 2 & .69 & $<.01$ & $<.02$ \\
\hline BIP & Green Sunfish & 07-24-1991 & Fish, WB & 206 & 74.2 & 8 & .1 & $<2$ & .38 & $<.01$ & $<.02$ \\
\hline BIP & Corixids, mixed & 08-01-1991 & Invertebrate & 20 & 83.5 & 180 & 6 & 15 & 3.7 & $<.01$ & .44 \\
\hline BO & Algae & 08-11-1993 & Vegetation & 22 & 69.6 & 9,560 & 4.55 & $<4.69$ & 181 & .691 & .238 \\
\hline DP & Common Carp & $06-25-1991$ & Fish, WB & 3,094 & 78.0 & 120 & .3 & $<2$ & 5.2 & $<.01$ & $<.02$ \\
\hline DP & Common Carp & 06-25-1991 & Fish, WB & 2,506 & 78.2 & 180 & .32 & $<2$ & 5.2 & $<.01$ & $<.02$ \\
\hline DP & Flannelmouth Sucker & $06-25-1991$ & Fish, WB & 741 & 76.4 & 572 & .62 & $<2$ & 5.1 & .03 & $<.02$ \\
\hline DP & Corixids, mixed & 08-01-1991 & Invertebrate & 9 & 85.9 & 160 & .58 & 6 & 42.1 & $<.03$ & .45 \\
\hline KSP & Filamentous Green Algae & 08-10-1993 & Vegetation & 26 & 87.8 & 770 & 8.57 & 186 & 80.5 & $<.239$ & 2.20 \\
\hline KSP & Stonewart & 08-10-1993 & Vegetation & 26 & 81.9 & 281 & 3.15 & 11.6 & 136 & $<.210$ & .669 \\
\hline KSP & Green Sunfish & 08-10-1993 & Fish, WB & 177 & 72.6 & 48.9 & $<.491$ & 6.46 & .803 & $<.099$ & $<.099$ \\
\hline KSP & Pondweed & 08-10-1993 & Vegetation & 30 & 89.3 & 247 & 2.34 & 584 & 40.6 & $<.238$ & 3.23 \\
\hline KSP & Cattail & 08-10-1993 & Vegetation & 329 & 92.0 & 1,240 & 4.84 & 20.4 & 32.3 & $<.242$ & 1.03 \\
\hline LJP & Filamentous Green Algae & 08-10-1993 & Vegetation & 28 & 86.2 & 517 & 10.1 & 280 & 54.8 & $<.216$ & $<.216$ \\
\hline LJP & Stonewart & 08-10-1993 & Vegetation & 84 & 81.2 & 296 & 5.56 & 42.1 & 65.2 & $<.196$ & $<.196$ \\
\hline LJP & Ruppia & 08-10-1993 & Vegetation & 41 & 85.8 & 204 & 3.75 & 323 & 45.1 & $<.238$ & $<.238$ \\
\hline L6 & Corixids & 08-01-1991 & Invertebrate & 17 & 78.8 & 63 & .4 & $<2$ & 9.9 & $<.01$ & .089 \\
\hline L6 & Red Shiner & 07-31-1991 & Fish, WB & 137 & 71.3 & 5 & .1 & $<2$ & 7 & $<.01$ & $<.02$ \\
\hline MG & Watercress & 08-03-1993 & Vegetation & 163 & 91.9 & 528 & 3.3 & 34.6 & 19.3 & $<.243$ & .958 \\
\hline MMM & Coleoptera & 08-21-1991 & Invertebrate & 27 & 72.7 & 81 & .3 & 11 & 1.8 & $<.01$ & .07 \\
\hline MSR & Domestic Goose & 05-15-1991 & Egg & 104 & 68.8 & $<3$ & $<.1$ & 3 & 1.8 & $<.01$ & $<.03$ \\
\hline NR & Cattail & 06-25-1991 & Vegetation & 650 & 90.7 & - & - & - & - & - & - \\
\hline NR & Cattail & 06-25-1991 & Vegetation & 781 & 91.7 & - & - & - & - & - & - \\
\hline NR & Cattail & 06-25-1991 & Vegetation & 716 & 88.8 & - & - & - & - & - & - \\
\hline NR & Cattail & 06-25-1991 & Vegetation & 186 & 81.2 & - & - & - & - & - & - \\
\hline NR & Cattai & 06-25-1991 & Vegetation & 602 & 91.4 & - & - & - & - & - & - \\
\hline NR & Cattail & 06-25-1991 & Vegetation & 647 & 79.9 & - & - & - & - & - & - \\
\hline NR & Cattail & 06-25-1991 & Vegetation & 494 & 90.9 & - & - & - & - & - & - \\
\hline NR & Cattail & $06-25-1991$ & Vegetation & 437 & 92.5 & - & - & - & - & - & - \\
\hline NR & Cattail & $06-25-1991$ & Vegetation & 549 & 92.1 & - & - & - & - & - & - \\
\hline NR & Cattail & 06-25-1991 & Vegetation & 590 & 93.1 & - & - & - & - & - & - \\
\hline NR & Cattail & 06-25-1991 & Vegetation & 676 & 92.1 & - & - & - & - & - & - \\
\hline NR & Cattail & $06-25-1991$ & Vegetation & 563 & 90.7 & - & - & - & - & - & - \\
\hline NR & Fathead Minnow & 07-30-1991 & Fish, WB & 25 & 81.3 & 24 & .1 & $<2$ & 20.2 & $<.01$ & .03 \\
\hline NR & Red Shiner & 07-30-1991 & Fish, WB & 35 & 80.2 & 74 & .2 & $<2$ & 26.9 & $<.01$ & .05 \\
\hline NR & Corixids & 08-06-1991 & Invertebrate & 24 & 85.0 & 28 & .4 & $<2$ & 44.4 & $<.01$ & .49 \\
\hline GWP & Common Carp & 06-24-1991 & Fish, WB & 3,145 & 78.6 & 35 & .2 & $<2$ & 3.8 & $<.01$ & $<.02$ \\
\hline GWP & Common Carp & 06-24-1991 & Fish, WB & 6,123 & 73.8 & 52 & .2 & $<2$ & 2.4 & $<.01$ & $<.02$ \\
\hline GPJ & American Coot & 06-26-1991 & Egg & 23 & 79.9 & $<3$ & $<.1$ & 4 & 6.5 & $<.01$ & $<.02$ \\
\hline GWP & Corixids, mixed & 08-01-1991 & Invertebrate & 14 & 82.4 & 190 & .4 & 5 & 5.1 & $<.01$ & .23 \\
\hline S3 & Common Carp & 07-25-1991 & Fish, WB & 250 & 78.6 & 48 & .52 & $<2$ & 4.2 & $<.01$ & .078 \\
\hline S3 & Common Carp & $07-25-1991$ & Fish, WB & 255 & 79.7 & 180 & .8 & $<2$ & 3.2 & .01 & .1 \\
\hline S3 & Corixids & 08-06-1991 & Invertebrate & 13 & 86.3 & 260 & 1.4 & 2 & 10.3 & .01 & 2.45 \\
\hline S5 & Cattail & 08-01-1991 & Vegetation & 95 & 94.0 & - & - & - & - & - & - \\
\hline S5 & Cattail & 08-01-1991 & Vegetation & 191 & 90.9 & - & - & - & - & - & - \\
\hline S5 & Cattail & 08-01-1991 & Vegetation & 129 & 94.9 & - & - & - & - & - & - \\
\hline S5 & Cattail & 08-01-1991 & Vegetation & 100 & 93.9 & - & - & - & - & - & - \\
\hline
\end{tabular}


Table 35. Concentration of trace elements in biological samples collected from selected sites in the middle Green River basin,eastern Utah (except Stewart Lake Waterfowl Management Area), 1991-93_Continued

\begin{tabular}{|c|c|c|c|c|c|c|c|c|c|c|c|c|c|}
\hline $\begin{array}{l}\text { Short site } \\
\text { name }\end{array}$ & $\begin{array}{c}\text { Chromium } \\
(\mu \mathrm{g} / \mathrm{g})\end{array}$ & $\begin{array}{l}\text { Copper } \\
(\mu \mathrm{g} / \mathrm{g})\end{array}$ & $\begin{array}{l}\text { Iron } \\
(\mu \mathrm{g} / \mathrm{g})\end{array}$ & $\begin{array}{c}\text { Mercury } \\
(\mu \mathrm{g} / \mathrm{g})\end{array}$ & $\begin{array}{l}\text { Magnesium } \\
\quad(\mu \mathrm{g} / \mathrm{g})\end{array}$ & $\begin{array}{c}\text { Manga- } \\
\text { nese } \\
(\mu \mathrm{g} / \mathrm{g})\end{array}$ & $\begin{array}{c}\text { Molyb- } \\
\text { denum } \\
(\mu \mathrm{g} / \mathrm{g})\end{array}$ & $\begin{array}{l}\text { Nickel } \\
(\mu \mathrm{g} / \mathrm{g})\end{array}$ & $\begin{array}{l}\text { Lead } \\
(\mu \mathrm{g} / \mathrm{g})\end{array}$ & $\begin{array}{l}\text { Selenium } \\
(\mu \mathrm{g} / \mathrm{g})\end{array}$ & $\begin{array}{l}\text { Strontium } \\
(\mu \mathrm{g} / \mathrm{g})\end{array}$ & $\begin{array}{c}\text { Vanadium } \\
(\mu \mathrm{g} / \mathrm{g})\end{array}$ & $\begin{array}{c}\text { Zinc } \\
(\mu \mathrm{g} / \mathrm{g})\end{array}$ \\
\hline BCD & 1.6 & 3.4 & 101 & .3 & 1,410 & 21.2 & $<1$ & .75 & $<.4$ & 9.8 & 107 & .3 & 88.1 \\
\hline BCD & 4.1 & 3.9 & 1,390 & .16 & 2,030 & 68.7 & $<1$ & 2 & 1 & 12.0 & 36.3 & 6.8 & 78.9 \\
\hline BC149 & 3.3 & 5.3 & 6,340 & .02 & 4,850 & 2,710 & 2 & 7.9 & 1 & 8.7 & 287 & 11 & 44.9 \\
\hline BC149 & 1.1 & 3.8 & 236 & .26 & 1,510 & 28 & $<1$ & .57 & $<.5$ & 19.0 & 135 & 1 & 129 \\
\hline BC149 & 3.6 & 2.6 & 1,190 & .007 & 2,440 & 257 & $<1$ & 2.2 & .6 & .7 & 96.9 & 3.3 & 15 \\
\hline BC149 & 2 & 7 & 335 & .11 & 1,490 & 18 & $<1$ & 1.2 & $<.4$ & 43.0 & 67.3 & 1.1 & 61.5 \\
\hline BIP & 2.1 & 2.1 & 55 & .44 & 1,410 & 21.9 & $<1$ & 1.2 & $<.5$ & 9.4 & 405 & $<.3$ & 87 \\
\hline BIP & .66 & 2.5 & 42 & .64 & 1,360 & 20.8 & $<1$ & .4 & $<.5$ & 9.8 & 297 & $<.3$ & 75.5 \\
\hline BIP & .45 & 19.4 & 238 & .2 & 1,500 & 27.9 & $<1$ & .4 & $<.5$ & 5.2 & 158 & .5 & 208 \\
\hline BO & 22.6 & 17.0 & 12,300 & $<.094$ & 9,780 & 440 & $<4.69$ & 16.2 & 13.2 & .61 & 155 & 8.14 & 48.0 \\
\hline DIP & .89 & 3.4 & 165 & .25 & 1,470 & 5.9 & $<1$ & .55 & $<.5$ & 17 & 368 & .6 & 180 \\
\hline DIP & .62 & 3.2 & 220 & .28 & 1,390 & 6.9 & $<1$ & .4 & $<.5$ & 14 & 271 & .7 & 182 \\
\hline DIP & 1.4 & 2.7 & 491 & .25 & 1,480 & 13 & $<1$ & .92 & $<.5$ & 13 & 163 & 1.6 & 60.2 \\
\hline DIP & $<.3$ & 11 & 290 & .11 & 1,430 & 33.6 & $<1$ & .8 & $<1$ & 8.1 & 37.2 & .5 & 363 \\
\hline KSP & 3.15 & 3.89 & 1,460 & $<.25$ & 5,530 & 492 & $<1.20$ & 3.29 & 1.50 & 3.55 & 622 & 2.99 & 25.6 \\
\hline KSP & 1.51 & 2.87 & 294 & $<.197$ & 6,470 & 222 & $<1.05$ & 2.49 & 7.02 & 2.99 & 1220 & $<1.05$ & 8.06 \\
\hline KSP & 20.2 & 6.67 & 233 & .208 & 1,540 & 8.51 & $<.495$ & 1.05 & 1.14 & 29.7 & 159 & $<.495$ & 209 \\
\hline KSP & 3.76 & 5.10 & 630 & $<.237$ & 5,260 & 582 & 1.78 & 4.12 & $<1.19$ & 3.41 & 267 & 2.13 & 38.6 \\
\hline KSP & 8.76 & 6.56 & 6,730 & $<.249$ & 5,080 & 143 & $<1.21$ & 5.24 & 2.01 & 3.21 & 111 & 7.36 & 63.7 \\
\hline LJP & 2.56 & 2.66 & 1,670 & $<.223$ & 14,200 & 1,340 & $<1.08$ & 3.77 & 4.64 & 1.99 & 842 & 3.8 & 16.6 \\
\hline LJP & 2.24 & 1.58 & 787 & $<.219$ & 8,820 & 961 & $<.980$ & 1.47 & 5.03 & 2.92 & 1,920 & 1.45 & 9.52 \\
\hline LJP & 1.90 & 1.99 & 565 & $<.238$ & 12,200 & 2,040 & 1.25 & 2.43 & 3.20 & 1.69 & 775 & 2.02 & 23.4 \\
\hline L6 & .2 & 15 & 132 & .13 & 844 & 27 & $<1$ & .3 & .6 & 1.7 & 52.1 & $<.3$ & 143 \\
\hline L6 & 1 & 2.4 & 49 & .23 & 1,250 & 11 & $<1$ & .71 & $<.4$ & 4.3 & 103 & $<.3$ & 163 \\
\hline MG & 3.02 & 11.3 & 3,430 & $<.214$ & 5,680 & 270 & 1.60 & 4.28 & $<1.21$ & 58.1 & 216 & 5.87 & 59.1 \\
\hline MMM & 1.5 & 14 & 170 & .031 & 1,240 & 66 & $<1$ & .97 & $<.4$ & 9.5 & 55.9 & $<.3$ & 184 \\
\hline MSR & .55 & 3.2 & 106 & $<.01$ & 539 & .5 & $<1$ & .4 & $<.1$ & 6.7 & 10 & $<.3$ & 51.9 \\
\hline NR & - & - & - & - & - & - & - & - & - & 13 & - & - & - \\
\hline NR & - & - & - & - & - & - & - & - & - & 39 & - & - & - \\
\hline NR & - & - & - & - & - & - & - & - & - & 7.4 & - & - & - \\
\hline NR & - & - & - & - & - & - & - & - & - & 15 & - & - & - \\
\hline NR & - & - & - & - & - & - & - & - & - & 11 & - & - & - \\
\hline NR & - & - & - & - & - & - & - & - & - & $<.2$ & - & - & - \\
\hline NR & - & - & - & - & - & - & - & - & - & 17 & - & - & - \\
\hline NR & - & - & - & - & - & - & - & - & - & 15 & - & - & - \\
\hline NR & - & - & - & - & - & - & - & - & - & 23 & - & - & - \\
\hline NR & - & - & - & - & - & - & - & - & - & 12 & - & - & - \\
\hline NR & - & - & - & - & - & - & - & - & - & 13 & - & - & - \\
\hline NR & - & - & - & - & - & - & - & - & - & 18 & - & - & - \\
\hline NR & 2.5 & 4.6 & 106 & .01 & 1,530 & 9 & $<1$ & 1.5 & $<.4$ & 100 & 299 & $<.3$ & 190 \\
\hline NR & 2.2 & 7.4 & 133 & .01 & 1,570 & 15 & $<1$ & 1.2 & $<.4$ & 81 & 307 & .3 & 175 \\
\hline NR & $<.1$ & 25 & 132 & .055 & 1,210 & 46.4 & $<1$ & .2 & $<.5$ & 35 & 49.2 & $<.3$ & 118 \\
\hline GWP & 2.1 & 2.8 & 112 & .18 & 1,360 & 7.2 & $<1$ & 1.2 & $<.5$ & 12 & 364 & .5 & 156 \\
\hline GWP & .95 & 3.6 & 127 & .31 & 1,200 & 10 & $<1$ & .5 & $<.5$ & 7.7 & 343 & .6 & 193 \\
\hline GPJ & 3 & 4.3 & 113 & .055 & 876 & 2.2 & $<1$ & 1.6 & $<.1$ & 18 & 39.1 & $<.3$ & 65.7 \\
\hline GWP & .3 & 18 & 253 & .25 & 1,240 & 23.9 & $<1$ & .4 & $<.4$ & 8.1 & 79.5 & .5 & 137 \\
\hline S3 & 5.3 & 4.6 & 121 & .04 & 1,270 & 6.9 & $<1$ & 2.5 & $<.4$ & 7.1 & 311 & .4 & 178 \\
\hline S3 & 1.3 & 4.2 & 245 & .036 & 1,120 & 8.7 & $<1$ & .65 & $<.4$ & 8.2 & 132 & .5 & 152 \\
\hline S3 & .43 & 19.3 & 399 & .074 & 1,420 & 35.2 & $<1$ & .77 & $<.4$ & 5.2 & 290 & .6 & 194 \\
\hline S5 & - & - & - & - & - & - & - & - & - & .3 & - & - & - \\
\hline S5 & - & - & - & - & - & - & - & - & - & .5 & - & - & - \\
\hline S5 & - & - & - & - & - & - & - & - & - & .75 & - & - & - \\
\hline S5 & - & - & - & - & - & - & - & - & - & .78 & - & - & - \\
\hline
\end{tabular}


Table 35. Concentration of trace elements in biological samples collected from selected sites in the middle Green River basin,eastern Utah (except Stewart Lake Waterfowl Management Area), 1991-93-Continued

\begin{tabular}{|c|c|c|c|c|c|c|c|c|c|c|c|}
\hline $\begin{array}{c}\text { Short site } \\
\text { name }\end{array}$ & Species & Date & Sample type & $\begin{array}{l}\text { Total } \\
\text { weight } \\
\text { (g) }\end{array}$ & $\begin{array}{l}\text { Percent } \\
\text { moisture }\end{array}$ & $\begin{array}{c}\text { Alumi- } \\
\text { num } \\
(\mu \mathrm{g} / \mathrm{g})\end{array}$ & $\begin{array}{c}\text { Arsenic } \\
(\mu \mathrm{g} / \mathrm{g})\end{array}$ & $\begin{array}{l}\text { Boron } \\
(\mu \mathrm{g} / \mathrm{g})\end{array}$ & $\begin{array}{c}\text { Barium } \\
(\mu \mathrm{g} / \mathrm{g})\end{array}$ & $\begin{array}{l}\text { Beryllium } \\
(\mu \mathrm{g} / \mathrm{g})\end{array}$ & $\underset{(\mu \mathrm{g} / \mathrm{g})}{\text { Cadmium }}$ \\
\hline$\overline{\text { S5 }}$ & Cattail & 08-01-1991 & Vegetation & 193 & 92.9 & - & - & - & - & - & - \\
\hline S5 & Cattail & 08-01-1991 & Vegetation & 289 & 93.0 & - & - & - & - & - & - \\
\hline S5 & Cattail & 08-01-1991 & Vegetation & 137 & 77.5 & - & - & - & - & - & - \\
\hline S5 & Cattail & 08-01-1991 & Vegetation & 207 & 90.3 & - & - & - & - & - & - \\
\hline S5 & Cattail & 08-01-1991 & Vegetation & 88 & 83.7 & - & - & - & - & - & - \\
\hline S5 & Cattail & 08-01-1991 & Vegetation & 146 & 92.6 & - & - & - & - & - & - \\
\hline S5 & Common Carp & 07-31-1991 & Fish, WB & 158 & 78.4 & 85 & .46 & $<2$ & 4.2 & .01 & .05 \\
\hline S5 & Corixids & 08-06-1991 & Invertebrate & 5 & 73.6 & 170 & 1.5 & $<2$ & 10.9 & $<.01$ & .15 \\
\hline S5 & Common Carp & 08-08-1991 & Fish, WB & 212 & 79.4 & 58 & .4 & $<2$ & 4 & $<.01$ & .13 \\
\hline SR & Cattail & 06-25-1991 & Vegetation & 701 & 91.3 & - & - & - & - & - & - \\
\hline SR & Cattail & $06-25-1991$ & Vegetation & 1,182 & 88.4 & - & - & - & - & - & - \\
\hline SR & Cattail & 06-25-1991 & Vegetation & 965 & 84.6 & - & - & - & - & - & - \\
\hline SR & Cattail & $06-25-1991$ & Vegetation & 869 & 91.3 & - & - & - & - & - & - \\
\hline SR & Cattail & $06-25-1991$ & Vegetation & 623 & 90.8 & - & - & - & - & - & - \\
\hline SR & Cattail & $06-25-1991$ & Vegetation & 561 & 93.8 & - & - & - & - & - & - \\
\hline SR & Cattail & 06-25-1991 & Vegetation & 395 & 93 & - & - & - & - & - & - \\
\hline SR & Red Shiner & 07-30-1991 & Fish, WB & 22 & 77.9 & 56 & .3 & $<2$ & 48.4 & $<.01$ & .03 \\
\hline SR & Coleoptera larvae & 08-06-1991 & Invertebrate & 9 & 84.2 & 38 & 3.2 & $<2$ & 1.6 & $<.01$ & .02 \\
\hline SR & Corixids & 08-06-1991 & Invertebrate & 28 & 78.9 & 63 & .3 & $<2$ & 5.9 & $<.01$ & .16 \\
\hline SB & Colorado Pikeminnow & 08-10-1993 & Fish, WB & 11 & 76.5 & 135 & $<.5$ & $<.548$ & 6.81 & $<.110$ & .179 \\
\hline WSP & Mixed, mostly amphipoda & 08-21-1991 & Invertebrate & 28 & 95.5 & 1,860 & 2.4 & 81 & 15.5 & .05 & .81 \\
\hline WSP & American Coot & 07-12-1991 & Egg & 21 & 77.0 & $<3$ & $<.1$ & 6 & .63 & $<.01$ & $<.02$ \\
\hline WSP & Gadwall & 06-06-1991 & Egg & 42 & 65.2 & $<3$ & $<.1$ & $<2$ & 2.6 & $<.01$ & $<.02$ \\
\hline WSP & Gadwall & 06-06-1991 & Egg & 36 & 68.7 & $<3$ & $<.1$ & $<2$ & 2.1 & $<.01$ & .02 \\
\hline WSP & Gadwall & 06-11-1991 & Egg & 40 & 68.2 & $<3$ & $<.1$ & $<2$ & 6 & $<.01$ & $<.02$ \\
\hline WSP & Gadwall & 06-11-1991 & Egg & 34 & 69.0 & $<3$ & $<.1$ & $<2$ & 4.8 & $<.01$ & $<.03$ \\
\hline WSP & Gadwall & 06-20-1991 & Egg & 34 & 67.9 & $<3$ & $<.1$ & $<2$ & 4.8 & $<.01$ & $<.02$ \\
\hline WSP & Gadwall & 06-27-1991 & Egg & 37 & 68.4 & $<3$ & $<.1$ & $<2$ & 9.2 & $<.01$ & $<.03$ \\
\hline WSP & Gadwall & 07-07-1991 & Egg & 39 & 68.5 & $<3$ & $<.1$ & $<2$ & 1.5 & $<.01$ & $<.03$ \\
\hline WSP & Gadwall & 07-10-1991 & Egg & 39 & 67.9 & $<3$ & $<.1$ & $<2$ & 4.1 & $<.01$ & $<.03$ \\
\hline WSP & Gadwall & 07-10-1991 & Egg & 39 & 68.5 & $<3$ & $<.1$ & $<2$ & 6.4 & $<.01$ & $<.02$ \\
\hline WSP & Mallard & 05-14-1991 & Egg & 43 & 69.9 & $<3$ & $<.1$ & $<2$ & 2.9 & $<.01$ & $<.02$ \\
\hline WSP & Mallard & 05-14-1991 & Egg & 48 & 69.0 & $<3$ & $<.1$ & $<2$ & 2.6 & $<.01$ & $<.02$ \\
\hline WSP & Green Sunfish & 07-23-1991 & Fish, WB & 166 & 80.7 & 38 & $<.2$ & 8 & .48 & $<.01$ & $<.02$ \\
\hline WSP & Common Carp & 08-12-1991 & Fish, WB & 858 & 76.0 & 200 & .2 & 4 & 2 & $<.01$ & .03 \\
\hline WSP & Common Carp & 07-23-1991 & Fish, WB & 3,384 & 76.1 & 110 & .3 & 4 & 1.2 & $<.01$ & .04 \\
\hline WSP & Common Carp & 07-23-1991 & Fish, WB & 3,622 & 73.5 & 150 & .4 & 2 & 1.7 & $<.01$ & $<.02$ \\
\hline WSP & Pondweed & 08-21-1991 & Vegetation & 95 & 84.0 & 2,160 & 1.5 & 1,650 & 25 & .079 & .13 \\
\hline
\end{tabular}


Table 35. Concentration of trace elements in biological samples collected from selected sites in the middle Green River basin,eastern Utah (except Stewart Lake Waterfowl Management Area), 1991-93-Continued

\begin{tabular}{|c|c|c|c|c|c|c|c|c|c|c|c|c|c|}
\hline $\begin{array}{c}\text { Short site } \\
\text { name }\end{array}$ & $\begin{array}{l}\text { Chromium } \\
(\mu \mathrm{g} / \mathrm{g})\end{array}$ & $\begin{array}{c}\text { Copper } \\
(\mu \mathrm{g} / \mathrm{g})\end{array}$ & $\begin{array}{l}\text { Iron } \\
(\mu \mathrm{g} / \mathrm{g})\end{array}$ & $\begin{array}{c}\text { Mercury } \\
(\mu \mathrm{g} / \mathrm{g})\end{array}$ & $\begin{array}{l}\text { Magnesium } \\
(\mu \mathrm{g} / \mathrm{g})\end{array}$ & $\begin{array}{c}\text { Manga- } \\
\text { nese } \\
(\mu \mathrm{g} / \mathrm{g})\end{array}$ & $\begin{array}{l}\text { Molyb- } \\
\text { denum } \\
(\mu \mathrm{g} / \mathrm{g})\end{array}$ & $\begin{array}{l}\text { Nickel } \\
(\mu \mathrm{g} / \mathrm{g})\end{array}$ & $\begin{array}{l}\text { Lead } \\
(\mu \mathrm{g} / \mathrm{g})\end{array}$ & $\begin{array}{l}\text { Selenium } \\
(\mu \mathrm{g} / \mathrm{g})\end{array}$ & $\begin{array}{l}\text { Strontium } \\
(\mu \mathrm{g} / \mathrm{g})\end{array}$ & $\underset{(\mu \mathrm{g} / \mathrm{g})}{\text { Vanadium }}$ & $\begin{array}{c}\text { Zinc } \\
(\mu g / g)\end{array}$ \\
\hline$\overline{\text { S5 }}$ & - & - & - & - & - & - & - & - & - & .5 & - & - & - \\
\hline S5 & - & - & - & - & - & - & - & - & - & .81 & - & - & - \\
\hline S5 & - & - & - & - & - & - & - & - & - & .5 & - & - & - \\
\hline S5 & - & - & - & - & - & - & - & - & - & .2 & - & - & - \\
\hline S5 & - & - & - & - & - & - & - & - & - & $<.2$ & - & - & - \\
\hline S5 & - & - & - & - & - & - & - & - & - & $<.2$ & - & - & - \\
\hline S5 & 4 & 3.5 & 152 & .042 & 1,290 & 7.3 & $<1$ & 2.2 & $<.5$ & 8.5 & 343 & .5 & 155 \\
\hline S5 & .49 & 16 & 212 & .049 & 891 & 55.3 & $<1$ & .53 & 1.6 & 8 & 277 & .4 & 72.5 \\
\hline S5 & 2.2 & 4.8 & 172 & .045 & 1,210 & 16 & $<1$ & 1.3 & $<.5$ & 12 & 194 & .4 & 131 \\
\hline SR & - & - & - & - & - & - & - & - & - & 27 & - & - & - \\
\hline SR & - & - & - & - & - & - & - & - & - & 12 & - & - & - \\
\hline SR & - & - & - & - & - & - & - & - & - & 12 & - & - & - \\
\hline SR & - & - & - & - & - & - & - & - & - & 14 & - & - & - \\
\hline SR & - & - & - & - & - & - & - & - & - & 9.9 & - & - & - \\
\hline SR & - & - & - & - & - & - & - & - & - & 41 & - & - & - \\
\hline SR & - & - & - & - & - & - & - & - & - & 9 & - & - & - \\
\hline SR & 4.5 & 6.5 & 124 & .009 & 1,350 & 9.3 & $<1$ & 2.4 & $<.4$ & 87 & 231 & $<.3$ & 144 \\
\hline SR & .2 & 23.4 & 79 & .02 & 2,020 & 34.3 & $<1$ & .2 & $<.4$ & 44 & 13.1 & $<.3$ & 180 \\
\hline SR & .2 & 20.5 & 127 & .11 & 877 & 19 & $<1$ & .3 & .5 & 12 & 93.2 & $<.3$ & 224 \\
\hline SB & 27.3 & 28.5 & 406 & .283 & 1,700 & 19.8 & $<.548$ & 2.15 & 2.22 & 3.28 & 129 & $<.548$ & 168 \\
\hline WSP & 2.5 & 11 & 1,200 & .078 & 6,810 & 45.7 & $<1$ & 2.2 & $<2$ & 60.0 & 274 & 5.6 & 529 \\
\hline WSP & $<.1$ & 3.6 & 137 & .17 & 431 & 2.2 & $<1$ & $<.1$ & $<.1$ & 27 & 14.1 & $<.3$ & 70.4 \\
\hline WSP & $<.1$ & 3.3 & 109 & .2 & 337 & 4.8 & $<1$ & $<.1$ & $<.1$ & 9 & 7.7 & $<.3$ & 61.1 \\
\hline WSP & $<.1$ & 5 & 162 & .86 & 388 & 2 & 2 & $<.1$ & $<.1$ & 9.5 & 8.1 & $<.3$ & 64 \\
\hline WSP & $<.1$ & 4.3 & 138 & .32 & 383 & 1 & $<1$ & $<.1$ & $<.1$ & 2.8 & 9.73 & $<.3$ & 64.8 \\
\hline WSP & $<.1$ & 3.4 & 127 & .34 & 369 & 1.7 & $<1$ & $<.2$ & $<.1$ & 8 & 11.3 & $<.3$ & 58.5 \\
\hline WSP & $<.1$ & 3.9 & 101 & .57 & 311 & 1.1 & $<1$ & .2 & .1 & 12 & 11.9 & $<.3$ & 69 \\
\hline WSP & $<.1$ & 4.2 & 147 & .38 & 358 & 1 & $<1$ & $<.2$ & .1 & 3.7 & 14.9 & $<.3$ & 64.3 \\
\hline WSP & $<.1$ & 3.7 & 132 & .96 & 348 & .94 & $<1$ & .2 & $<.1$ & 9.5 & 8.3 & $<.3$ & 60.3 \\
\hline WSP & $<.1$ & 3.9 & 126 & .42 & 373 & 1.1 & $<1$ & $<.2$ & $<.1$ & 5.5 & 12.4 & $<.3$ & 58.9 \\
\hline WSP & $<.1$ & 3.7 & 111 & .63 & 345 & 1.2 & $<1$ & $<.2$ & $<.1$ & 3.8 & 13 & $<.3$ & 53.7 \\
\hline WSP & $<.1$ & 4 & 121 & .092 & 437 & 1 & $<1$ & $<.1$ & $<.1$ & 20 & 15.5 & $<.3$ & 59.7 \\
\hline WSP & $<.1$ & 3.1 & 104 & .12 & 407 & 1.7 & $<1$ & $<.1$ & $<.1$ & 19 & 14.8 & $<.3$ & 55.1 \\
\hline WSP & .59 & 1.3 & 103 & .13 & 1,500 & 21.8 & $<1$ & .5 & $<.4$ & 100.0 & 87 & $<.3$ & 107 \\
\hline WSP & .96 & 4.7 & 272 & .031 & 1,460 & 22.3 & $<1$ & .52 & $<.4$ & 77.0 & 182 & .5 & 219 \\
\hline WSP & 1.9 & 3.3 & 162 & .031 & 1,300 & 12 & $<1$ & 1 & $<.4$ & 78.0 & 147 & .4 & 214 \\
\hline WSP & 1.8 & 2.6 & 189 & .026 & 1,280 & 14 & $<1$ & 1 & $<.4$ & 83.0 & 159 & .4 & 205 \\
\hline WSP & 3.6 & 4.4 & 1,430 & .02 & 8,800 & 421 & 1 & 12 & 1 & 6.5 & 530 & 7.3 & 22 \\
\hline
\end{tabular}


Table 36. Concentration of selenium in muscle plugs from endangered fish from Stewart Lake Waterfowl Management Area near Jensen, Utah, 19972000

[Data from U.S. Fish and Wildlife Service; mm, millimeters; g, grams; $\mu \mathrm{g} / \mathrm{g}$, micrograms per gram, dry weight; —, no data]

\begin{tabular}{|c|c|c|c|c|c|c|}
\hline Site name & $\begin{array}{l}\text { Abbreviated } \\
\text { site name }\end{array}$ & Date & Species & $\begin{array}{l}\text { Length } \\
(\mathrm{mm})\end{array}$ & $\begin{array}{l}\text { Weight } \\
\text { (g) }\end{array}$ & $\begin{array}{l}\text { Selenium } \\
(\mu \mathrm{g} / \mathrm{g})\end{array}$ \\
\hline Stewart Inlet & SLI & $05-31-2000$ & Colorado Pikeminnow & 460 & 941 & 4.75 \\
\hline Stewart Lake & SLM & 06-02-1999 & Colorado Pikeminnow & - & - & 5.31 \\
\hline Stewart Lake & SLM & 06-02-1999 & Colorado Pikeminnow & - & - & 8.40 \\
\hline Stewart Center & SLM & 06-19-1997 & Razorback Sucker & 568 & 1,720 & 12.1 \\
\hline Stewart Lake & SLM & 06-22-1999 & Razorback Sucker & 403 & 703 & 3.18 \\
\hline J3 Drain & SLN & 06-10-1997 & Colorado Pikeminnow & - & - & 8.14 \\
\hline Stewart North Overlook & SLN & $06-27-2000$ & Razorback Sucker & 315 & 391 & 5.76 \\
\hline Stewart Outlet & SLO & 06-02-1998 & Colorado Pikeminnow & 422 & 618 & 5.84 \\
\hline Stewart Outlet & SLO & 06-10-1997 & Colorado Pikeminnow & 575 & - & 7.40 \\
\hline Stewart Outlet & SLO & 06-18-1997 & Razorback Sucker & 485 & 1,014 & 13.8 \\
\hline Stewart Outlet & SLO & 06-19-1997 & Razorback Sucker & 529 & 1,420 & 18.2 \\
\hline Stewart Outlet & SLO & 06-19-1997 & Razorback Sucker & 505 & 1,410 & 21.5 \\
\hline Stewart Outlet & SLO & $06-28-2000$ & Razorback Sucker & 315 & 356 & 5.02 \\
\hline Stewart Outlet & SLO & $06-28-2000$ & Razorback Sucker & 312 & 382 & 6.56 \\
\hline Stewart Outlet & SLO & $06-28-2000$ & Razorback Sucker & 271 & 250 & 9.84 \\
\hline Stewart Outlet & SLO & 07/10-2000 & Razorback Sucker & 330 & 421 & 9.44 \\
\hline Stewart Outlet & SLO & 07/10-2000 & Razorback Sucker & 340 & 469 & 9.48 \\
\hline Stewart Outlet & SLO & $07 / 12-2000$ & Razorback Sucker & 330 & 392 & 8.17 \\
\hline Stewart Outlet & SLO & 07/12-2000 & Razorback Sucker & 368 & 565 & 9.06 \\
\hline
\end{tabular}


Table 37. Concentration of selenium in muscle plugs from endangered fish from selected sites in the middle Green River basin, eastern Utah (except Stewart Lake Waterfowl Management Area), 1995-2000

[mm, millimeters; $\mu \mathrm{g} / \mathrm{g}$, micrograms per gram, dry weight; - , no data]

\begin{tabular}{|c|c|c|c|c|c|c|}
\hline Site name & $\begin{array}{l}\text { Abbreviated } \\
\text { site name }\end{array}$ & Date & Species & $\begin{array}{l}\text { Length } \\
(\mathrm{mm})\end{array}$ & $\begin{array}{l}\text { Weight } \\
\text { (grams) }\end{array}$ & $\begin{array}{c}\text { Selenium } \\
(\mu \mathrm{g} / \mathrm{g})\end{array}$ \\
\hline Bonanza Bridge & BB & 03-17-1999 & Colorado Pikeminnow & 563 & 1,319 & 3.05 \\
\hline Bonanza Bridge & BB & 08-04-1999 & Razorback Sucker & 420 & 740 & .069 \\
\hline Bonanza Bridge & BB & 08-04-1999 & Colorado Pikeminnow & 534 & 1,040 & 2.73 \\
\hline Bonanza Bridge & BB & 08-04-1999 & Colorado Pikeminnow & 497 & 848 & 3.56 \\
\hline Bonanza Bridge & BB & 08-04-1999 & Colorado Pikeminnow & 552 & 1,085 & \\
\hline Bonanza Bridge & BB & 08-12-1998 & Colorado Pikeminnow & 448 & 561 & 3.38 \\
\hline Bonanza Bridge & BB & 08-29-1995 & Colorado Pikeminnow & 515 & 1,050 & 4.20 \\
\hline Bonanza Bridge & $\mathrm{BB}$ & 08-29-1995 & Colorado Pikeminnow & 550 & 1,145 & 4.70 \\
\hline Bonanza Bridge & BB & 08-29-1995 & Colorado Pikeminnow & 510 & 1,037 & 5.30 \\
\hline Brush Creek & $\mathrm{BC}$ & $04-30-1996$ to $05-16-1996$ & Colorado Pikeminnow & - & - & 3.71 \\
\hline Brush Creek & $\mathrm{BC}$ & 05-06-1997 & Colorado Pikeminnow & 632 & 2,300 & 4.19 \\
\hline Brush Creek & $\mathrm{BC}$ & 07-30-1996 & Colorado Pikeminnow & 440 & 740 & 4.02 \\
\hline Brush Creek & $\mathrm{BC}$ & 08-13-1998 & Colorado Pikeminnow & 405 & 488 & 4.09 \\
\hline Bonanza & $\mathrm{BO}$ & 03-17-1999 & Colorado Pikeminnow & 540 & 1,172 & 2.20 \\
\hline Bonanza & $\mathrm{BO}$ & 07-19-1995 & Colorado Pikeminnow & 476 & - & 4.80 \\
\hline Bonanza & $\mathrm{BO}$ & 07-19-1995 & Colorado Pikeminnow & 560 & - & 6.50 \\
\hline Bonanza & $\mathrm{BO}$ & 07-19-1995 & Colorado Pikeminnow & 602 & - & 7.20 \\
\hline Bonanza & $\mathrm{BO}$ & 08-03-1999 & Razorback Sucker & 415 & 718 & .74 \\
\hline Bonanza & $\mathrm{BO}$ & 08-05-1999 & Razorback Sucker & 458 & 923 & .73 \\
\hline Bonanza & $\mathrm{BO}$ & 08-08-2000 & Colorado Pikeminnow & 510 & 1,081 & 4.41 \\
\hline Collier Draw & $\mathrm{CD}$ & $03-08-2000$ & Colorado Pikeminnow & 481 & 777 & 2.70 \\
\hline Collier Draw & $\mathrm{CD}$ & 03-16-1999 & Colorado Pikeminnow & 544 & 1,276 & 3.51 \\
\hline Collier Draw & $\mathrm{CD}$ & 07-28-1999 & Razorback Sucker ${ }^{1}$ & - & 496 & .48 \\
\hline Collier Draw & $\mathrm{CD}$ & 08-07-1997 & Colorado Pikeminnow & 621 & 1,798 & 4.22 \\
\hline Collier Draw & $\mathrm{CD}$ & 08-09-2000 & Colorado Pikeminnow & 480 & 885 & 6.01 \\
\hline Collier Draw & $\mathrm{CD}$ & 08-09-2000 & Colorado Pikeminnow & 480 & 739 & 6.87 \\
\hline Collier Draw & $\mathrm{CD}$ & 08-12-1998 & Colorado Pikeminnow & 500 & 927 & 2.94 \\
\hline Collier Draw & $\mathrm{CD}$ & 08-12-1998 & Colorado Pikeminnow & 524 & 984 & 3.08 \\
\hline Escalante Bar & $\mathrm{EB}$ & 04-30-1997 & Razorback Sucker & 547 & 1,860 & 10.7 \\
\hline Escalante Bar & EB & 05-07-1997 & Razorback Sucker & 508 & 1,300 & 26.4 \\
\hline Escalante Bar & EB & 08-03-1999 & Razorback Sucker & 371 & - & .63 \\
\hline Hamacker Bottom & HA & 03-14-2000 & Colorado Pikeminnow & 487 & 893 & 3.32 \\
\hline Hamacker Bottom & HA & 03-16-1999 & Colorado Pikeminnow & 443 & - & 3.00 \\
\hline Hamacker Bottom & HA & 03-16-1999 & Colorado Pikeminnow & 571 & - & 3.06 \\
\hline Hamacker Bottom & HA & 03-16-1999 & Colorado Pikeminnow & 333 & - & 3.46 \\
\hline Hamacker Bottom & HA & 07-27-1999 & Razorback Sucker ${ }^{1}$ & - & 358 & .69 \\
\hline Hamacker Bottom & HA & 08-04-1999 & Colorado Pikeminnow & 487 & 964 & \\
\hline Hamacker Bottom & HA & 08-09-2000 & Colorado Pikeminnow & 600 & 1,474 & 3.64 \\
\hline Hamacker Bottom & HA & 08-09-2000 & Colorado Pikeminnow & 564 & 1,300 & 6.91 \\
\hline Hamacker Bottom & HA & 08-12-1998 & Colorado Pikeminnow & 649 & 2,106 & 1.65 \\
\hline Hamacker Bottom & HA & 08-12-1998 & Colorado Pikeminnow & 510 & 1,004 & 2.40 \\
\hline Horseshoe Bend & $\mathrm{HB}$ & 03-08-2000 & Colorado Pikeminnow & 553 & 1,229 & 5.41 \\
\hline Horseshoe Bend & $\mathrm{HB}$ & 08-09-2000 & Colorado Pikeminnow & 478 & 769 & 5.24 \\
\hline Leota Bottom & LB & $04-30-1996$ to $05-16-1996$ & Colorado Pikeminnow & - & - & 3.59 \\
\hline Leota Bottom & LB & $04-30-1996$ to $05-16-1996$ & Colorado Pikeminnow & - & - & 4.95 \\
\hline
\end{tabular}


Table 37. Concentration of selenium in muscle plugs from endangered fish from selected sites in the middle Green River basin, eastern Utah (except Stewart Lake Waterfowl Management Area), 1995-2000_Continued

\begin{tabular}{|c|c|c|c|c|c|c|}
\hline Site name & $\begin{array}{l}\text { Abbreviated } \\
\text { site name }\end{array}$ & Date & Species & $\begin{array}{l}\text { Length } \\
(\mathrm{mm})\end{array}$ & $\begin{array}{l}\text { Weight } \\
\text { (grams) }\end{array}$ & $\begin{array}{c}\text { Selenium } \\
(\mu \mathrm{g} / \mathrm{g})\end{array}$ \\
\hline Leota Bottom & LB & 03-18-1999 & Colorado Pikeminnow & 498 & 873 & 2.34 \\
\hline Leota Bottom & LB & 03-18-1999 & Colorado Pikeminnow & 597 & 1,470 & 2.41 \\
\hline Leota Bottom & LB & 03-18-1999 & Colorado Pikeminnow & 636 & 1,752 & 2.87 \\
\hline Leota Bottom & LB & 03-18-1999 & Colorado Pikeminnow & 592 & 1,506 & 4.13 \\
\hline Leota Bottom & LB & 04-29-1997 & Colorado Pikeminnow & 551 & 1,550 & 2.66 \\
\hline Leota Bottom & LB & 04-29-1997 & Colorado Pikeminnow & 605 & 1,850 & 3.31 \\
\hline Leota Bottom & LB & 04-29-1997 & Colorado Pikeminnow & 538 & 1,145 & 3.48 \\
\hline Leota Bottom & LB & 05-02-1997 & Colorado Pikeminnow & 524 & 1,275 & 2.78 \\
\hline Leota Bottom & LB & 05-02-1997 & Colorado Pikeminnow & 601 & 1,740 & 3.33 \\
\hline Leota Bottom & LB & 07-28-1999 & Razorback Sucker ${ }^{1}$ & - & 463 & .80 \\
\hline Leota Bottom & LB & 08-05-1999 & Razorback Sucker & 370 & 497 & .67 \\
\hline Leota Bottom & LB & 08-13-1998 & Colorado Pikeminnow & 565 & 1,229 & 2.77 \\
\hline WB- Old Charlie & $\mathrm{OCW}$ & 06-23-1995 & Razorback Sucker & 531 & 1,636 & 4.20 \\
\hline WB- Old Charlie & $\mathrm{OCW}$ & 06-26-1995 & Razorback Sucker & 585 & 2,353 & 5.00 \\
\hline WB- Old Charlie & OCW & 06-27-1995 & Razorback Sucker & 471 & 1,182 & 3.10 \\
\hline WB- Old Charlie & OCW & 06-27-1995 & Razorback Sucker & 556 & 1,909 & 3.40 \\
\hline WB- Old Charlie & OCW & 06-27-1995 & Razorback Sucker & 572 & 1,909 & 4.10 \\
\hline WB- Old Charlie & OCW & 06-27-1995 & Razorback Sucker & 545 & 2,000 & 4.40 \\
\hline Ouray NFH & $\mathrm{OFH}$ & 06-09-2000 & Razorback Sucker & - & - & .89 \\
\hline Ouray NFH & $\mathrm{OFH}$ & 06-09-2000 & Razorback Sucker & - & - & .89 \\
\hline Ouray NFH & $\mathrm{OFH}$ & 06-09-2000 & Razorback Sucker & - & - & .94 \\
\hline Ouray NFH & $\mathrm{OFH}$ & 06-09-2000 & Razorback Sucker & - & - & .95 \\
\hline Ouray NFH & $\mathrm{OFH}$ & 06-09-2000 & Razorback Sucker & - & - & .95 \\
\hline RZB & RZB & $04-30-1996$ to $05-16-1996$ & Colorado Pikeminnow & - & - & 4.92 \\
\hline RZB & RZB & 04-26-1999 & Colorado Pikeminnow & 470 & 845 & 3.51 \\
\hline RZB & RZB & 05-06-1997 & Razorback Sucker & 539 & 1,675 & 3.76 \\
\hline RZB & RZB & 05-06-1997 & Colorado Pikeminnow & 374 & 450 & 4.20 \\
\hline RZB & RZB & 05-15-1995 & Razorback Sucker & 440 & 950 & 6.20 \\
\hline RZB & RZB & 05-16-1995 & Razorback Sucker & 449 & 1,125 & 2.90 \\
\hline RZB & RZB & 06-02-1997 & Razorback Sucker & 329 & 260 & 1.46 \\
\hline RZB & RZB & 06-02-1997 & Razorback Sucker & 452 & 860 & 7.61 \\
\hline Stewart/Ashley & SA & 05-06-1997 & Colorado Pikeminnow & 623 & 1,625 & 5.43 \\
\hline Stewart/Ashley & SA & 05-06-1997 & Razorback Sucker & 489 & 1,300 & 6.34 \\
\hline Stewart/Ashley & SA & 05-07-1997 & Razorback Sucker & 591 & 2,100 & 5.15 \\
\hline Stewart/Ashley & SA & 05-07-1997 & Colorado Pikeminnow & 627 & 1,580 & 5.45 \\
\hline Stewart/Ashley & SA & 05-12-1997 & Colorado Pikeminnow & 568 & 1,500 & 4.91 \\
\hline Stewart/Ashley & SA & 08-03-1999 & Colorado Pikeminnow & 533 & 1,171 & 5.06 \\
\hline Sheppard Bottom & SB & 03-18-1999 & Colorado Pikeminnow & 586 & 1,245 & 2.39 \\
\hline Sheppard Bottom & SB & 03-18-1999 & Colorado Pikeminnow & 429 & 534 & 3.08 \\
\hline Sheppard Bottom & SB & 03-18-1999 & Colorado Pikeminnow & 607 & 1,563 & \\
\hline Sheppard Bottom & SB & 03-18-1999 & Colorado Pikeminnow & 486 & 747 & \\
\hline Sheppard Bottom & SB & 07-30-1999 & Colorado Pikeminnow ${ }^{1}$ & - & 515 & 1.88 \\
\hline Sheppard Bottom & SB & 07-31-1996 & Colorado Pikeminnow & 540 & 1,102 & 2.99 \\
\hline Sheppard Bottom & SB & 07-31-1996 & Colorado Pikeminnow & 564 & 1,297 & 4.57 \\
\hline Sheppard Bottom & SB & 08-05-1999 & Razorback Sucker & 398 & 625 & 1.37 \\
\hline
\end{tabular}

\footnotetext{
${ }^{1}$ Salvaged sample from dead fish.
} 